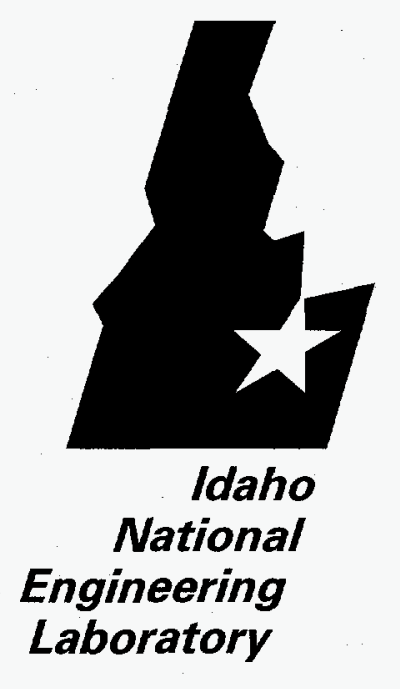

INEL-96/0473

February 1997

\title{
Enhanced Integrated Nonthermal Treatment System Study
}

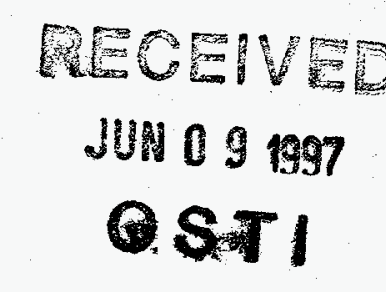

Charles Biagi

Bill Schwinkendorf

Ben Teheranian 
INEL-96/0473

\title{
Enhanced \\ Integrated Nonthermal Treatment System Study
}

\author{
Charles Biagi \\ Bill Schwinkendorf \\ Ben Teheranian
}

February 1997

Idaho National Engineering Laboratory

Lockheed Martin Idaho Technologies Company

Idaho Falls, ID 83415

Prepared for the

U.S. Department of Energy

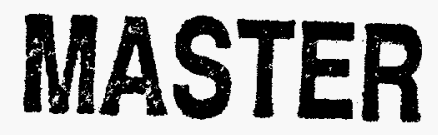

Idaho Operations Office

Under DOE Idaho Field Office

Contract No. DE-AC07-94ID13223

DISTRIBUTION OF THIS DOCUMENT IS UNLIMITED 


\section{CONTENTS}

ACRONYMS AND ABBREVIATIONS $\ldots \ldots \ldots \ldots \ldots \ldots \ldots \ldots \ldots \ldots \ldots$

EXECUTIVE SUMMARY $\ldots \ldots \ldots \ldots \ldots \ldots \ldots \ldots \ldots \ldots \ldots \ldots \ldots$

1. INTRODUCTION $\ldots \ldots \ldots \ldots \ldots \ldots \ldots \ldots \ldots \ldots \ldots \ldots \ldots \ldots \ldots \ldots \ldots \ldots$

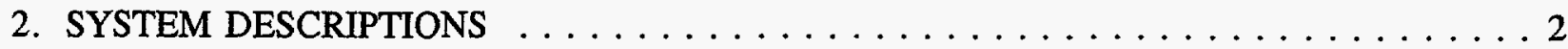

2.1 System ENTS-1: Catalytic Wet Oxidation, Desorption and Vitrification . . . . . . 4

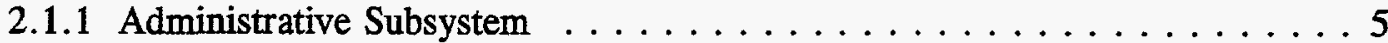

2.1.2 Receiving and Preparation Subsystem ................ 5

2.1.3 Aqueous Waste Treatment Subsystem . . . . . . . . . . . . . . . 6

2.1.4 Catalytic Wet Oxidation Organic Destruction Subsystem . . . . . . . . . 7

2.1.5 Process Residue and Inorganic Sludge Treatment Subsystem . . . . . . . . 8

2.1.6 Bulk Soil Treatment Subsystem . . . . . . . . . . . . . . . . . . . . 9

2.1.7 Debris Treatment Subsystem . . . . . . . . . . . . . . . . 10

2.1 .8 Metal Melting Subsystem . . . . . . . . . . . . . . . . 12

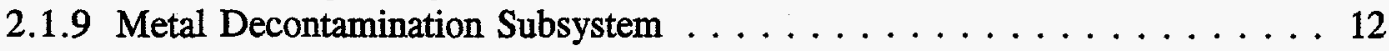

2.1 .10 Lead Recovery Subsystem . . . . . . . . . . . . . . . . 12

2.1 .11 Mercury Amalgamation Subsystem . . . . . . . . . . . . . 12

2.1.12 Special Waste Treatment Subsystem . . . . . . . . . . . . 13

2.1 .13 Polymer Stabilization Subsystem $\ldots \ldots \ldots \ldots \ldots \ldots \ldots$

2.1.14 Air Pollution Control (APC) Subsystem . . . . . . . . . . . . . . 13

2.1.15 Vitrification Subsystem . . . . . . . . . . . . . . . . . 14

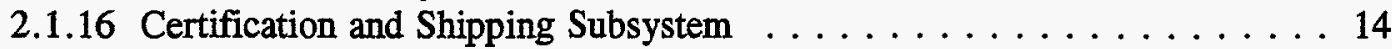

2.1.17 Support Subsystem . . . . . . . . . . . . . . . . . . 15

2.2 System ENTS-2: Catalytic Wet Oxidation and Vitrification for Process Residues ... 16

2.3 System ENTS-3: Catalytic Wet Oxidation and Polymer Stabilization . . . . . . . 17

2.4 System ENTS-4: Catalytic Wet Oxidation, Desorption, Compaction and Polymer

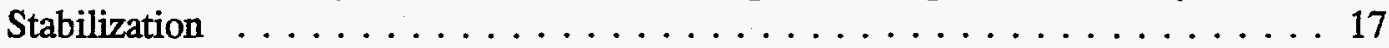

2.4.1 Claytec Subsystem . . . . . . . . . . . . . . . . 17

2.5 System ENTS-5: Catalytic Wet Oxidation with Increased Reaction Rates . . . . . . 18

2.6 Rationale for System Selection and Design . . . . . . . . . . . . . . . 19

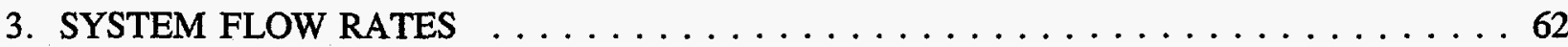

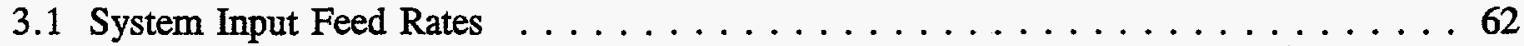

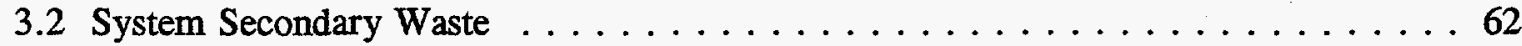

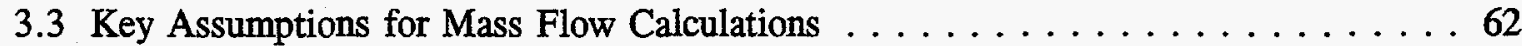

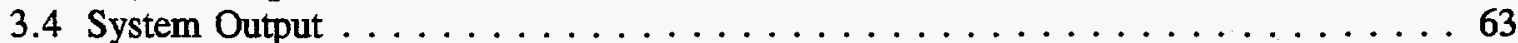

3.5 Energy Balance Calculation Results . . . . . . . . . . . . . . . . 63

3.6 Mass Balance Calculation Results . . . . . . . . . . . . . . . 63

4. TOTAL LIFE CYCLE COST ESTIMATES $\ldots \ldots \ldots \ldots \ldots \ldots \ldots \ldots \ldots$

4.1 Estimating Methods, Basis, and Assumptions $\ldots \ldots \ldots \ldots \ldots \ldots \ldots$

4.2 Treatment Facility TLCC (with Disposal) Estimate Summaries . . . . . . . . . 78

4.3 TLCC Estimates (without Disposal) for ENTS-1, ENTS-2,

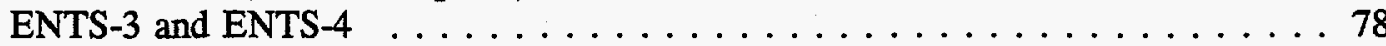

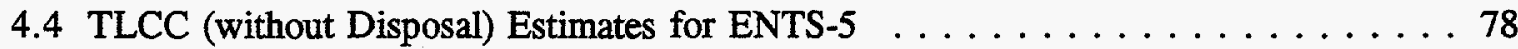

4.4.1 Reaction Rate Calculations . . . . . . . . . . . . . . . . 79 


\section{CONTENTS (Continued)}

4.4.2 Equipment Requirements . . . . . . . . . . . . . . . 79

4.4 .3 Labor Requirements . . . . . . . . . . . . . . . . 80

4.4 .4 Building Requirements . . . . . . . . . . . . . . 80

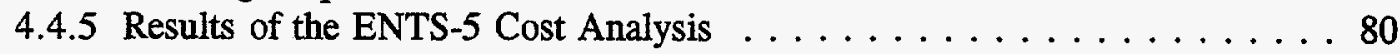

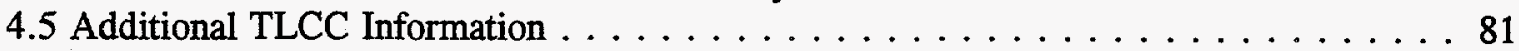

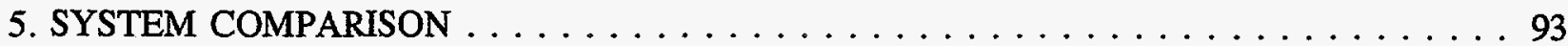

5.1 Feed Rates . . . . . . . . . . . . . . . . . . . . . . . 94

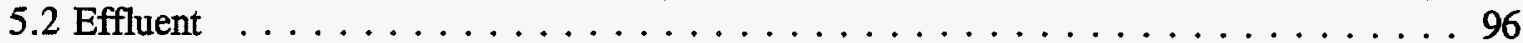

5.3 Waste Sent to Disposal . . . . . . . . . . . . . . . . . . . . . 97

5.4 Resources Used ... . . . . . . . . . . . . . . . . . . . . . 97

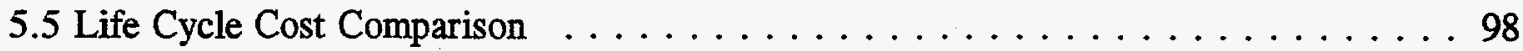

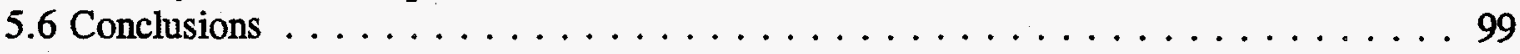

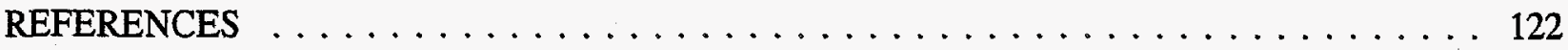

APPENDICES

Appendix A Figures

Appendix B Cost Data

\section{TABLES}

2-1. Summary System Descriptions for Enhanced Nonthermal Treatment System . . . . . . . 3

3-1. Input feed rates to ENTS subsystems $(\mathrm{lb} / \mathrm{hr}) \ldots \ldots \ldots \ldots \ldots$

3-2. Offgas, water and metal recycle outputs from the five ENTS systems $(\mathrm{lb} / \mathrm{hr}) \ldots \ldots \ldots 5$

3-3. Waste volume outputs from the five ENTS systems $\left(\mathrm{ft}^{3} / \mathrm{hr}\right) \ldots \ldots \ldots \ldots 6$

3-4. Energy Requirement for ENTS Systems (BTU/hr) $\ldots \ldots \ldots \ldots \ldots \ldots$

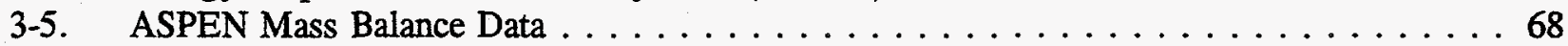

4-1. TLCC (without Disposal) Estimate for ENTS-1 (in millions of dollars) . . . . . . . 82

4-2. TLCC (without Disposal) Estimate for ENTS-2 (in millions of dollars) . . . . . . . . 83

4-3. TLCC (without Disposal) Estimate for ENTS-3 (in millions of dollars). . . . . . . . . 84

4-4. TLCC (without Disposal) Estimate for ENTS-4 (in millions of dollars) . . . . . . . . 85

4-5. TLCC (without Disposal) Estimate for ENTS-5 (in millions of dollars) . . . . . . . 86

4-6. ENTS TLCC Estimates with Disposal . . . . . . . . . . . . . . . 87

4-7. TLCC Comparisons of the five ENTS systems (in millions of dollars) . . . . . . . . 88

4-8. Normalized Lifecycle Cost Comparison of the Five ENTS Systems (normalized with respect to INTS System 5) (in millions of dollars) . . . . . . . . . . . . . . . . . . 89

4-9. TLCC Comparison (without disposal) by Subsystem for the Five ENTS systems (in millions

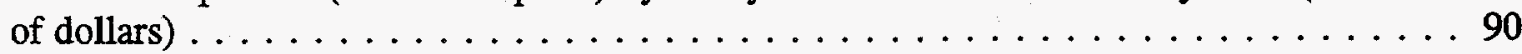

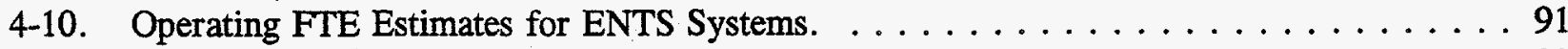

4-11. Reaction Rates for CWO. . . . . . . . . . . . . . . . . . . 92

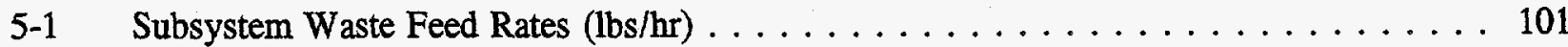

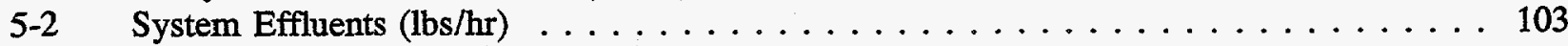

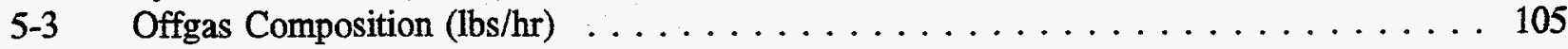




\section{DISCLAIMER}

This report was prepared as an account of work sponsored by an agency of the United States Government. Neither the United States Government nor any agency thereof, nor any of their employees, make any warranty, express or implied, or assumes any legal liability or responsibility for the accuracy, completeness, or usefulness of any information, apparatus, product, or process disclosed, or represents that its use would not infringe privately owned rights. Reference herein to any specific commercial product, process, or service by trade name, trademark, manufacturer, or otherwise does not necessarily constitute or imply its endorsement, recommendation, or favoring by the United States Government or any agency thereof. The views and opinions of authors expressed herein do not necessarily state or reflect those of the United States Government or any agency thereof. 


\section{DISCLAMMER}

Portions of this document may be illegible in electronic image products. Images are produced from the best available original document. 
5-4 Final Waste Form Mass sent to Disposal (lbs/hr) $\ldots \ldots \ldots \ldots \ldots$

5-5 Final Waste Form Volume Sent to Disposal $\left(\mathrm{ft}^{3} / \mathrm{hr}\right) \ldots \ldots \ldots \ldots \ldots$. . . . . . . . 107

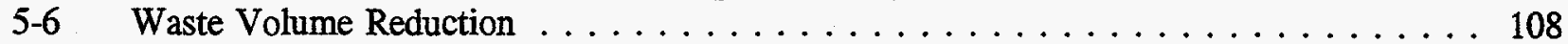

5-7 Treatment System Energy Cost . . . . . . . . . . . . . . . . . . . . . . . . . 109

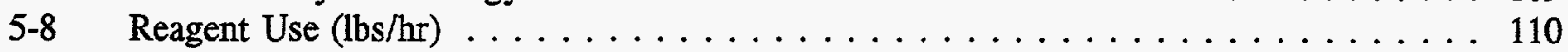

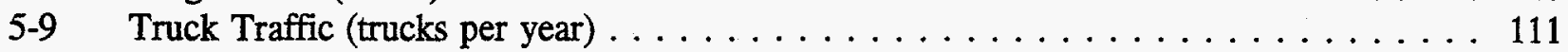

5-10 Personnel Requirements by Subsystem (Full Time Equivalents) . . . . . . . . . . . . 112

5-11 Total Life-Cycle Costs $(\$$ million $) \ldots \ldots \ldots \ldots \ldots \ldots \ldots \ldots \ldots \ldots$

5-12 Subsystem Life-Cycle Cost $(\$$ million $) \ldots \ldots \ldots \ldots \ldots \ldots \ldots$

\section{FIGURES}

2-1. ENTS System 1: PFD for Overall System $\ldots \ldots \ldots \ldots \ldots \ldots \ldots \ldots \ldots$

2-2. ENTS System 1: Plan View of Overall System. . . . . . . . . . . . . 22

2-3. ENTS System 1: PFD for Receiving and Preparation Subsystem . . . . . . . . . . 23

2-4. ENTS System 1: Perspective View of Receiving and Unloading Area of the Receiving and Preparation Subsystem . . . . . . . . . . . . . . . . . . . . . . . 24

2-5. ENTS System 1: Perspective View of Decap, Sort, and Dump Cell of the Receiving and Preparation Subsystem . . . . . . . . . . . . . . . . . . . 25

2-6. ENTS System 1: PFD for Aqueous Waste Treatment Subsystem . . . . . . . . . . . 26

2-7. ENTS System 1: Perspective View of Aqueous Waste Treatment Subsystem . . . . . . . 27

2-8. ENTS System 1: PFD for the CWO Organic Destruction Subsystem . . . . . . . . . . 28

2-9. ENTS System 1: Perspective View of the CWO Organic Destruction Subsystem . . . . . 29

2-10. ENTS System 1: PFD for Process Residue and Inorganic Sludge Treatment Subsystem . . 30

2-11. ENTS System 1: Perspective View of Process Residue and Inorganic Sludge Treatment

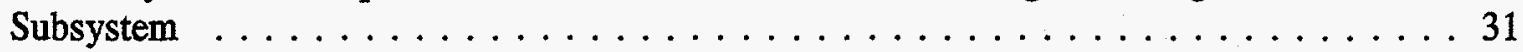

2-12. ENTS System 1: PFD for Bulk Soil Treatment Subsystem . . . . . . . . . . . . 32

2-13. ENTS System 1: Perspective View of Bulk Soil Treatment Subsystem . . . . . . . . 33

2-14. ENTS System 1: PFD for Soft Debris Treatment Subsystem . . . . . . . . . . . . . . 34

2-15. ENTS System 1: Perspective View of Soft Debris Treatment Subsystem . . . . . . 35

2-16. ENTS System 1: PFD for Open Debris Treatment Subsystem . . . . . . . . . . . . . 36

2-17. ENTS System 1: Perspective View of Open Debris Treatment Subsystem . . . . . . . . 37

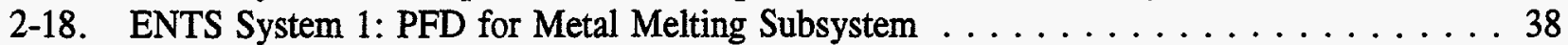

2-19. ENTS System 1: Perspective View of Metal Melting Subsystem . . . . . . . . . . . . 39

2-20. ENTS System 1: PFD for Metal Decontamination Subsystem . . . . . . . . . . . 40

2-21. ENTS System 1: Perspective View of Metal Decontamination Subsystem . . . . . . . . 41

2-22. ENTS System 1: PFD for Mercury Amalgamation and Lead Recovery Subsystems . . . . 42

2-23. ENTS System 1: Perspective View of Lead Recovery Subsystem . . . . . . . . . . . 43

2-24. ENTS System 1: Perspective View of Mercury Amalgamation Subsystem . . . . . . . . 44

2-25. ENTS System 1: PFD for Polymer Stabilization Subsystem . . . . . . . . . . . . . 45

2-26. ENTS System 1: Perspective View of Polymer Stabilization Subsystem . . . . . . . . . 46

2-27. ENTS System 1: PFD for Air Pollution Control Subsystem . . . . . . . . . . . . 47

2-28. ENTS System 1: Perspective View of Air Pollution Control Subsystem . . . . . . . . 48

2-29. ENTS System 1: PFD for Vitrification Subsystem . . . . . . . . . . . . . . 49

2-30. ENTS System 1: Perspective View of Vitrification Subsystem . . . . . . . . . . 50

2-31. ENTS System 1: PFD for Certification and Shipping Subsystem . . . . . . . . 51

2-32. ENTS System 1: Perspective View of Certification and Shipping Subsystem . . . . . . 52 


\section{CONTENTS (Continued)}

2-33. ENTS System 2: PFD for Overall System $\ldots \ldots \ldots \ldots \ldots \ldots \ldots \ldots \ldots \ldots$

2-34. ENTS System 2: Plan View of Overall System $\ldots \ldots \ldots \ldots \ldots \ldots \ldots \ldots$

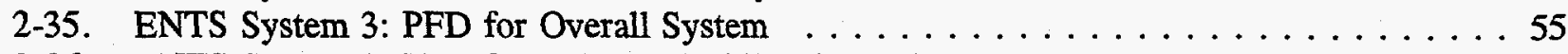

2-36. ENTS System 3: PFD for Polymer Stabilization Subsystem . . . . . . . . . . . 56

2-37. ENTS System 4: PFD for Overall System $\ldots \ldots \ldots \ldots \ldots \ldots \ldots \ldots \ldots$

2-38. ENTS System 4 : Plan View of Overall System $\ldots \ldots \ldots \ldots \ldots \ldots$

2-39. ENTS System 4: PFD for Claytec Subsystem . . . . . . . . . . . . . . . 59

2-40. ENTS System 4: Perspective View of Claytec Subsystem . . . . . . . . . . . . 60

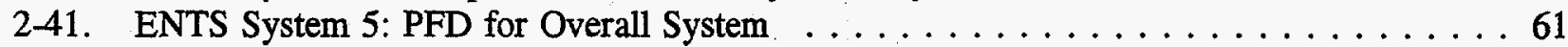

3-1. General mass flow rates $(\mathrm{lb} / \mathrm{hr})$ for system ENTS-1: Catalytic Wet Oxidation, Desorption and

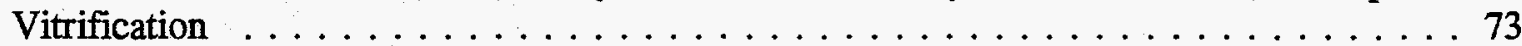

3-2. General mass flow rates (lb/hr) for system ENTS-2: Catalytic Wet Oxidation and Vitrification for Process Residues . . . . . . . . . . . . . . . . . . 74

3-3. General mass flow rates $(\mathrm{lb} / \mathrm{hr})$ for system ENTS-3: Catalytic Wet Oxidation and Polymer Stabilization . . . . . . . . . . . . . . . . . 75

3-4. General mass flow rates (lb/hr) for system ENTS-4: Catalytic Wet Oxidation, Desorption, Compaction and Polymer Stabilization . . . . . . . . . . . . . . 76

3-5. General mass flow rates (lb/hr) for system ENTS-5 : Catalytic Wet Oxidation with Increased

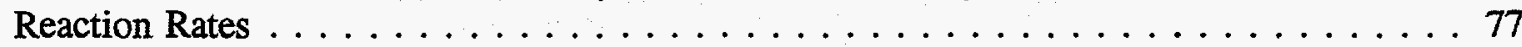

$5-1 \quad$ Offgas Emissions $\ldots \ldots \ldots \ldots \ldots \ldots \ldots \ldots \ldots \ldots \ldots \ldots \ldots \ldots \ldots$

$5-2 \quad$ Final Waste Form Volume $\ldots \ldots \ldots \ldots \ldots \ldots \ldots \ldots \ldots \ldots$

$5-3 \quad$ Volume Reduction $\ldots \ldots \ldots \ldots \ldots \ldots \ldots \ldots \ldots \ldots$

$5-4$ Total Life Cycle Cost $\ldots \ldots \ldots \ldots \ldots \ldots \ldots \ldots \ldots \ldots \ldots \ldots$ 


\section{ACRONYMS AND ABBREVIATIONS}

$\begin{array}{ll}\text { APC } & \text { air pollution control } \\ \text { CEM } & \text { continuous emissions monitoring } \\ \text { CWO } & \text { catalytic wet oxidation } \\ \text { DAF } & \text { dissolved air flotation } \\ \text { DOE } & \text { U.S. Department of Energy } \\ \text { EA } & \text { environmental assessment } \\ \text { EIS } & \text { environmental impact statement } \\ \text { ENTS } & \text { enhanced nonthermal treatment system } \\ \text { EPA } & \text { U.S. Environmental Protection Agency } \\ \text { F\&OR } & \text { functional and operational requirements } \\ \text { FTE } & \text { full-time equivalent } \\ \text { GPCR } & \text { gas phase corona reactor } \\ \text { gpm } & \text { gallons per minute } \\ \text { HEPA } & \text { high-efficiency particulate air (filter) } \\ \text { INEL } & \text { Idaho National Engineering Laboratory } \\ \text { NNTS } & \text { integrated nonthermal treatment system } \\ \text { ITTS } & \text { integrated thermal treatment system } \\ \text { LDPE } & \text { low density polyethylene } \\ \text { M } & \text { moles } \\ \text { MCC } & \text { motor control center } \\ \text { MEO } & \text { mediated electrochemical oxidation } \\ \text { MLLW } & \text { mixed low-level (radioactive) waste } \\ \text { OH• } & \text { hydroxyl radical } \\ \text { O\&M } & \text { operations and maintenance } \\ \text { PAN } & \text { passive/active neutron } \\ \text { PFD } & \text { process flow diagram } \\ \text { psi } & \text { pounds per square inch } \\ \text { psig } & \text { pounds per square inch, gage pressure } \\ \text { R\&D } & \text { research and development } \\ \text { RCRA } & \text { Resource Conservation and Recovery Act } \\ \text { ROM } & \text { rough order of magnitude } \\ \text { RTR } & \text { real-time radiography } \\ \text { SCC } & \text { secondary combustion chamber } \\ \text { SCM } & \text { system cost model } \\ \text { SGS } & \text { segmented gamma scanning } \\ \text { TCLP } & \text { Toxicity Characteristic Leaching Procedure } \\ \text { TDS } & \text { total dissolved solids } \\ \text { TLCC } & \text { total life cycle cost } \\ \text { TOC } & \text { total organic carbon } \\ \text { TRU } & \text { transuranic } \\ \text { UV } & \text { ultraviolet light } \\ \text { WBS } & \text { work breakdown structure } \\ \text { oC } & \text { degree } \\ \text { degree Centigrade } \\ \text { degree Fahrenheit } \\ \text { degree Kelvin } \\ \end{array}$




\section{EXECUTIVE SUMMARY}

The purpose of the Enhanced Nonthermal Treatment Systems (ENTS) study is to evaluate alternative configurations of one of the five systems evaluated in the Integrated Nonthermal Treatment Systems (INTS) study. Five alternative configurations are evaluated. Each is designed to enhance the final waste form performance by replacing grout with improved stabilization technologies, or to improve system performance by improving the destruction efficiency for organic contaminants. All enhanced systems are alternative configurations of System NT-5, which has the following characteristics:

\section{Nonthermal System NT-5:}

- catalytic wet oxidation (CWO) to treat organic material including organic liquids, sludges, and soft (or combustible) debris

- thermal desorption of inorganic sludge and process residue

- washing of soil and inorganic debris with treatment by CWO of removed organic material

- metal decontamination by abrasive blasting

- stabilization of treated sludge, soil, debris, and untreated debris with entrained contamination in grout

- stabilization of inorganic sludge, salts and secondary waste in polymer.

System NT-5 was chosen because it was designed to treat combustible debris thereby minimizing the final waste form volume, and because it uses grout for primary stabilization. The enhanced nonthermal systems were studied to determine the cost and performance impact of replacing grout (a commonly used stabilization agent in the DOE complex) with improved waste stabilization methods such as vitrification and polymer.

Two of the five enhanced nonthermal systems consist of a hybrid between two systems (nonthermal organic destruction is combined with thermal stabilization and metal recycling processes). In these two systems, grout is replaced with vitrification and metals with entrained contamination are melted into ingots for recycle. Two nonthermal enhanced systems were developed, in which grout is replaced with enhanced nonthermal stabilization processes. In the fifth system, the organic destruction rate is assumed to be increased by a factor of ten.

The characteristics of these enhanced nonthermal systems are as follows:

\section{ENTS-1:}

- catalytic wet oxidation of organic wastes and soft (combustible) debris

- washing of surface contaminated inorganic debris and soil and vitrification of treated debris and soil

- vacuum thermal desorption of inorganic sludges and polymer stabilization of treated sludges and secondary wastes

- melting of metal with entrained contamination and decontamination of surface contaminated metal for recycle

- decontamination or melting of lead for recycle

- retorting of mercury contaminated waste and amalgamation of mercury metal 


\section{ENTS-2: Same as ENTS-1 except}

- vacuum thermal desorption is eliminated

- untreated inorganic sludge and process residue are sent directly to a vitrifier

ENTS-3: Same as Nonthermal System NT-5 except

- stabilization of all treated wastes is by polymer; no grout stabilization is used.

ENTS-4: Same as Nonthermal System NT-5 except

- stabilization of all treated wastes is by compaction and sintering into pellets, with macroencapsulation of the pellets in polymer.

ENTS-5: Same as Nonthermal System NT-5 except

- the reaction rate for the CWO process that treats all organic wastes and soft debris is assumed to be increased by a factor of ten.

Mass balances, effluent, and cost data were developed for these systems based on conceptual system designs, process flow diagrams, and equipment layouts. The results of the enhanced nonthermal systems study.were compared with the results of previous nonthermal and thermal systems studies. The results of these studies indicate the following:

- Nonthermal and enhanced nonthermal systems cost approximately $\$ 1$ billion to $\$ 1.5$ billion more than thermal systems

- The mass of the offgas of nonthermal systems is one-third to one-tenth the mass of the offgas from thermal systems

- The final waste form volume of systems using vitrification is about one-third the volume of systems using nonthermal stabilization processes

Thus, there is no economic incentive to use nonthermal or enhanced nonthermal systems in place of thermal systems in a centralized treatment facility. However, decreased offgas and low final waste form volumes for hybrid systems may make these systems attractive from the standpoint of stakeholder acceptance.

Operating and maintenance costs are between 50 to 60 percent of the total life cycle cost for all systems. The major drivers for the higher treatment costs for nonthermal and enhanced nonthermal systems are the higher O\&M costs associated with more sorting and segregating of waste, higher volumes of waste passing through the certification and shipping subsystem, and more unit operations required to treat the waste.

System ENTS-2 has one-third the offgas from a system using a plasma furnace and one-tenth the offgas from a system using a rotary kiln with air for combustion, producing approximately the same final waste form volume at a life cycle cost of $\$ 1$ billion more than these thermal systems.

The costs for System ENTS-2 might be decreased by eliminating the washing process ( $\$ 153$ million) and treating all inorganic waste in the vitrifier. This should also decrease the front-end handling costs as less sorting will be required. However, a vitrification system must be demonstrated to reliably and efficiently destroy the organic contaminants in these inorganic matrices.

The more waste that can be vitrified, the lower the final waste form volume and the lower the disposal cost. Final waste form performance characteristics are enhanced with a vitrified product. 
The use of polymer to stabilize all waste (ENTS-3) decreases the final waste form volume slightly from that produced by System NT-5 which uses grout and polymer. Although there is little cost incentive to use all polymer, a waste form with better performance characteristics than grout may be produced.

Using hybrid waste forms (pellets macroencapsulated in polymer as in ENTS-4) may improve performance relative to System ENTS-3, which uses only polymer for stabilization, but at a cost of $\$ 550$ million. If the compressed and sintered pellets can pass TCLP without macroencapsulation, then the stabilization and disposal costs should decrease and be closer to those of System ENTS-3.

There is no cost incentive to perform $R \& D$ to increase the reaction rate of nonthermal organic destruction processes. Increasing the reaction rate will allow treatment of all organic wastes in the same reaction vessel, and in a shorter period of time, thereby decreasing the capital and operating costs of treatment. However, this savings is offset by the increase in material handling and maintenance costs associated with equipment and personnel required to handle the increased throughput, and the cost of equipment required to attain the increased reaction rate. However, an advantage of an increased reaction rate may be to increase the destruction efficiency if the residence time were kept the same as the original residence time used at the lower reaction rate (i.e., the throughput remains the same).

Nonthermal systems might be made more competitive with thermal systems by enhancing them through the use of vitrification for all inorganic wastes (except metals and secondary waste salts), and by eliminating the organic separation processes (thermal desorption and washing). This would decrease the capital and O\&M costs required to operate and maintain this equipment. Also, sorting requirements, and therefore the front-end handling costs, should decrease. The final waste form volume, and therefore certification and shipping costs and disposal costs, should also decrease. If these enhancements were implemented, the only nonthermal process would be destruction of organic waste, including soft (combustible) debris. Detailed evaluation of a hybrid system such as this, and comparison with selected thermal systems, would quantify how competitive a hybrid system can become. 


\section{INTRODUCTION}

The purpose of the Enhanced Nonthermal Treatment Systems (ENTS) study is to evaluate alternative configurations of the five systems evaluated in the Integrated Nonthermal Treatment Systems (INTS) study (Biagi, 1997). The configurations of alternatives that are evaluated are designed to improve the final waste form performance by replacing grout with improved stabilization technologies, or to improve system performance by improving the destruction efficiency for organic contaminants. All enhanced nonthermal systems are alternative configurations of a single nonthermal system to allow comparison of these alternative configurations.

Nonthermal system NT-5, which has the following characteristics, was chosen as the baseline for evaluation and against which comparisons would be made:

- catalytic wet oxidation (CWO) to treat organic material including organic liquids, sludges, and soft (or combustible) debris

- thermal desorption of inorganic sludge and process residue

- washing of soil and inorganic debris with CWO treatment of the removed organic material

- metal decontamination by abrasive blasting

- stabilization of treated sludge, soil, debris, and untreated debris with entrained contamination in grout

- stabilization of inorganic sludge, salts and secondary waste in polymer

System NT-5 was chosen over Systems NT-1, NT-2 and NT-3 because these three systems were not designed to break down combustible debris. System NT-2 treats combustible debris by vacuum thermal desorption, which does not destroy the combustible matrix. The mediated electrochemical oxidation (MEO) process (Systems NT-1 and NT-3) can treat ion exchange resins and soluble cellulose, but it cannot destroy plastics, wood, and other insoluble combustible debris. System NT-5 with CWO was chosen because CWO destroys combustible debris and minimizes the stabilized waste volume sent to disposal. Detailed descriptions of the nonthermal systems are contained in the INTS report (Biagi, 1997).

System NT-5 with CWO was selected over NT-4. System NT-4 uses acid digestion to treat organic contaminants and combustible debris, and uses phosphate bonded ceramic instead of grout as a stabilization material for the final waste form. The ENTS study was designed in part to replace grout with improved final waste forms. System NT-4 could have been used as the baseline or as another enhanced nonthermal system alternative. However, the cost differences would be slight, and a single baseline system used throughout the ENTS study provides continuity for cost and performance comparisons.

For each ENTS configuration, documents were developed that include process flow diagrams (PFDs), layouts, system characteristics (such as throughput, effluent, and volume reductions) and life-cycle cost estimates. Layouts were not developed for Systems ENTS-3 and ENTS-5, because they are so similar to System INTS-5.

Section 2 describes the systems evaluated in this report and provides the rationale for selecting these systems for evaluation. Sections 3 and 4 present the results of the ENTS evaluations. In Section 5 the results of this study are compared to the results for representative thermal systems from the Integrated Thermal Treatment System (ITTS) study (Feizollahi, 1995) and nonthermal systems from the INTS study. Design figures, including PFDs and layouts, are presented in Section 2 and Appendix A. 


\section{SYSTEM DESCRIPTIONS}

All ENTS systems are variations of INTS System 5, Catalytic Wet Oxidation (CWO). Table 2-1 presents a summary of the five ENTS systems.

All ENTS systems evaluated require (1) the use of extensive characterization and sorting, (2) a Gas Phase Corona Reactor (GPCR) for the destruction of residual organic vapors passing through the offgas treatment, (3) surface decontamination of bulk metals and recyclables, and (4) photo-oxidation destruction of any residual organic materials in the aqueous treatment subsystem.

Each system consists of either 16 or 17 subsystems. Most of the subsystems are identical, or only slightly modified in each of the five system configurations. These subsystems are listed below. It should be noted that not all subsystems are used in each ENTS systems.

The following subsystems are used in all ENTS systems:

- Administration Subsystem

- Receiving and Preparation Subsystem

- Aqueous Waste Treatment Subsystem

- Catalytic Wet Oxidation Organic Destruction Subsystem

- Bulk Soil Treatment Subsystem

- Soft Debris Treatment Subsystem

- Open Debris Treatment Subsystem

- Mercury Amalgamation Subsystem

- Lead Recovery Subsystem

- Metal Decontamination Subsystem

- Special Waste Treatment Subsystem

- Air Pollution Control Subsystem

- Certification and Shipping Subsystem

- Support Subsystem

The following subsystems are used in some ENTS systems:

- Vitrification Subsystem

- Metal Melting Subsystem

- Process Residue and Inorganic Sludge Treatment Subsystem

- Polymer Stabilization Subsystem

- Claytec Subsystem

- Grout Stabilization Subsystem 
Table 2-1. Summary System Descriptions for Enhanced Nonthermal Treatment System

\begin{tabular}{|c|c|}
\hline Designation & System Description \\
\hline \multirow{9}{*}{$\begin{array}{l}\text { ENTS System } \\
1: \\
\text { CWO, } \\
\text { Desorption and } \\
\text { Vitrification }\end{array}$} & Catalytic wet oxidation of soft debris and organics \\
\hline & Washing of open debris and soil \\
\hline & Vacuum thermal desorption of inorganic sludges and process residue \\
\hline & Vitrification of treated non-metallic debris and treated soil \\
\hline & $\begin{array}{l}\text { Polymer stabilization of inorganic salt waste, treated inorganic sludges and process } \\
\text { residue }\end{array}$ \\
\hline & Metal melting of complex metal debris \\
\hline & Decontamination of open metal debris \\
\hline & $\begin{array}{l}\text { Mercury retort of mercury contaminated debris and mercury amalgamation of } \\
\text { elemental mercury }\end{array}$ \\
\hline & Lead decontamination or melting and casting of lead contaminated debris \\
\hline \multirow{8}{*}{$\begin{array}{l}\text { ENTS System } \\
2: \\
\text { CWO and } \\
\text { Vitrification }\end{array}$} & Catalytic wet oxidation of soft debris and organics \\
\hline & Washing of open debris and soil \\
\hline & $\begin{array}{l}\text { Vitrification of treated non-metallic debris, treated soil, untreated process residues } \\
\text { and inorganic sludge, and some secondary waste streams }\end{array}$ \\
\hline & Polymer stabilization of inorganic salt waste and some secondary waste streams \\
\hline & Metal melting of complex metal debris \\
\hline & Decontamination of open metal debris \\
\hline & $\begin{array}{l}\text { Mercury retort of mercury contaminated debris and mercury amalgamation of } \\
\text { elemental mercury }\end{array}$ \\
\hline & Lead decontamination or melting and casting of lead contaminated debris \\
\hline \multirow{7}{*}{$\begin{array}{l}\text { ENTS System } \\
3: \\
\text { CWO, } \\
\text { Desorption and } \\
\text { Polymer } \\
\text { Stabilization }\end{array}$} & Catalytic wet oxidation of soft debris and organics \\
\hline & Washing of open debris and soil \\
\hline & Vacuum thermal desorption of inorganic sludges and process residue \\
\hline & $\begin{array}{l}\text { Polymer stabilization of treated debris, treated soil, complex debris, inorganic salt } \\
\text { waste, treated inorganic sludge and secondary waste streams }\end{array}$ \\
\hline & Decontamination of open metal debris \\
\hline & Mercury amalgamation of elemental mercury \\
\hline & Lead decontamination of lead contaminated debris \\
\hline
\end{tabular}


Table 2-1. (continued) Summary System Descriptions for Enhanced Nonthermal Treatment System

\begin{tabular}{|c|c|}
\hline Designation & System Description \\
\hline \multirow{2}{*}{$\begin{array}{l}\text { ENTS System } \\
\text { 4: }\end{array}$} & Catalytic wet oxidation of soft debris and organics \\
\hline & Washing of open debris and soil \\
\hline \multirow{5}{*}{$\begin{array}{l}\text { CWO, } \\
\text { Desorption, } \\
\text { Compaction and } \\
\text { Polymer } \\
\text { Stabilization }\end{array}$} & Vacuum thermal desorption of inorganic sludges and process residue \\
\hline & $\begin{array}{l}\text { Compaction, pelletizing, and polymer stabilization using the Claytec process of } \\
\text { complex debris, treated soil, inorganic salt waste, treated inorganic sludge and } \\
\text { secondary waste streams }\end{array}$ \\
\hline & Decontamination of open metal debris \\
\hline & Mercury amalgamation of elemental mercury \\
\hline & Lead decontamination of lead contaminated debris \\
\hline \multirow{7}{*}{$\begin{array}{l}\text { ENTS System } \\
5: \\
\text { CWO, } \\
\text { Desorption, } \\
\text { Compaction and } \\
\text { Polymer } \\
\text { Stabilization }\end{array}$} & $\begin{array}{l}\text { Catalytic wet oxidation of soft debris and organics, at ten times the INTS reaction } \\
\text { rates }\end{array}$ \\
\hline & Washing of open debris and soil \\
\hline & Vacuum thermal desorption of inorganic sludges and process residue \\
\hline & Mercury amalgamation of elemental mercury \\
\hline & Lead decontamination of lead contaminated debris \\
\hline & $\begin{array}{l}\text { Polymer stabilization of inorganic salt waste, treated inorganic sludge and } \\
\text { secondary waste streams }\end{array}$ \\
\hline & Grout stabilization of treated debris, and treated soil \\
\hline
\end{tabular}

\subsection{System ENTS-1: Catalytic Wet Oxidation, Desorption and Vitrification}

Figure 2-1 presents the overall PFD. Figure 2-2 presents the plan view layout of System ENTS-1. The system consists of all structures, buildings, and equipment needed to accomplish the functional and operational requirements (F\&ORs). The organic destruction subsystem is catalytic wet oxidation. The process residue and inorganic sludge treatment subsystem uses vacuum desorption to remove organics prior to stabilization. Bulk soil is treated by aqueous wash. Soft debris is treated by catalytic wet oxidation. Open debris is treated by high pressure wash. Non-metallic complex debris is treated by vitrification. Metallic complex debris is treated by metal melting. Mercury contaminated debris is sent to a mercury retort, where mercury is removed from the debris. Elemental mercury from receiving and preparation and the separated mercury from the retort are amalgamated. The mercury free debris is sent to vitrification. The Air Pollution Control (APC) subsystem is based on dry filtration, wet offgas scrubbing and organic destruction in a GPCR. The vitrification subsystem replaces the grout subsystem used in the INTS systems, and is equipped with an APC subsystem that includes dry and wet offgas treatment trains. System ENTS-1 consists of sixteen subsystems which are described below. 


\subsubsection{Administrative Subsystem}

The administrative subsystem includes all administrative and analytical laboratory buildings required for waste management support functions. The administration subsystem is essentially the same for all five systems.

The administration subsystem incorporates all technical and administrative support functions needed to manage the operation of a waste management facility. These functions include: (1) security, (2) access control, including personnel decontamination (radioactive and hazardous), (3) maintenance of uncontaminated areas and equipment, (4) health physics and radiation badges, (5) facility access control, (6) sanitary facilities, (7) work control and persomel support, (8) internal and external (public relations) communications, (9) spill or emergency response provisions (hazardous and radioactive), (10) analytical laboratory, (11) environmental field sampling, and (12) environmental regulatory reporting, records management and overall site management.

The administrative subsystem maintains general interfaces with all treatment subsystems. Operation and Maintenance (O\&M) consumables include analytical supplies, office supplies, sanitary supplies, drums, and personal protective equipment.

\subsubsection{Receiving and Preparation Subsystem}

Figure 2-3 presents the PFD for the receiving and preparation subsystem. Figures $2-4$ and 2-5 present the subsystem's perspective view of this subsystem, which is the same for all systems.

This subsystem utilizes cranes and forklift trucks to unload waste containers from incoming vehicles. The physical state of the waste in the containers is identified by a real-time radiography (RTR) unit. A passive/active neutron (PAN) assay unit determines the level of transuranic (TRU) contamination of the waste. A segmented gamma scanning (SGS) unit is used to assay beta and gamma radioactivity. A computer software and bar code scanning unit records and tracks the wastes.

The wastes are classified either as those requiring or not requiring sorting. Containers of wastes not requiring sorting are moved directly to the appropriate treatment subsystem. For purposes of this study, approximately 75 percent of the waste was assumed to require sorting.

Waste containers requiring sorting are grouped into two categories: homogeneous waste (e.g. sludge) and heterogeneous waste (e.g. debris). For both categories, the container is decapped by a saw mounted on a gantry robot. After decapping, homogeneous waste not requiring size reduction is dumped into transport hoppers. Heterogeneous waste containers are emptied on a sorting table equipped with master-slave and hydraulic manipulators which are used for segregating materials into treatment types, including open debris, soft debris, complex debris, cemented process residue, soil, lead, ferrous metals, and special wastes.

Various sorting technologies, such as robotic assisted sorting tables, vibratory screens, and air classifiers, may be used in the sorting area. Waste identified by manifest or other documentation as contaminated with mercury compounds will be isolated from other debris. Waste classified as debris under the EPA Debris Rule (40 CFR 268.45) must be segregated from other wastes. To be classified as debris a minimum size is required. However, EPA does allow size reduction of debris if it is first cleaned of other material such as soil. The regulations state that cleaning must be performed until the debris appears free of non-debris material. The sorted waste material is size reduced by saw cutting and/or mechanical auger shredding, as required, for the treatment subsystem. Prepared sorted waste is placed in transfer bins and moved to the treatment subsystems. Nonmetallic containers are cut or shredded into smaller pieces. 


\subsubsection{Aqueous Waste Treatment Subsystem}

Figure 2-6 presents the PFD for the aqueous waste treatment subsystem. Figure 2-7 presents the subsystem's perspective view. This subsystem collects and treats input aqueous waste, which could include wastewater with corrosive properties, dissolved solids, suspended solids, radionuclides, and a broad range of RCRA-controlled toxic, heavy-metal, and organic contaminants. Waste is treated to meet the facilities recycling needs and/or discharge conditions.

This subsystem also treats secondary aqueous wastes such as (1) salt solutions from organic destruction, (2) filtrate from the aqueous wash and high pressure wash subsystems, (3) the aqueous waste from APC subsystem condensate and scrubber blowdown, (4) sludge from abrasive blasting in the metal decontamination subsystem, (5) rinse water from container wash down, and (6) water from equipment and floor drains.

The unit operations of the aqueous waste treatment subsystem are designed for maximum flexibility and can be used in series, in parallel, or as stand alone units. The selection of specific unit operations will be based on waste characterization from the receiving and preparation subsystem, as well as in-process evaluations. Operations staff will select the appropriate unit operations. Based on characterization, incoming waste will be segregated and stored in four batch tanks classified as having (1) high levels of total organic carbon (TOC), (2) high TDS levels, (2) low TDS levels, or (4) heavy metals/mercury containing liquids.

For high TOC waste, the primary approach is processing in the organic destruction subsystem. Alternatively, a treatment train is included in the aqueous waste treatment subsystem as a backup. Gross organic removal is accomplished in an oil/water separator or other flotation thickener. Suspended solids filtration is performed using back-flushable filters. Calcium removal prior to organic treatment is performed in a mixing reactor by the addition of sulfuric acid to achieve precipitation of calcium sulfate.

For low concentrations of dissolved organics, removal is accomplished in an ultraviolet (UV) reactor. The destruction of organics by oxidation to carbon dioxide and water is accomplished by exposing the aqueous solution to the light. The reactor design allows the addition of both ozone $\left(\mathrm{O}_{3}\right)$ and hydrogen peroxide $\left(\mathrm{H}_{2} \mathrm{O}_{2}\right)$. The UV light reacts with hydrogen peroxide or ozone to create hydroxyl radicals (OH•) which attack and break down the organics. Waste characterization prior to transfer to the reactor optimizes the use of ozone, UV, and hydrogen peroxide because treatment effectiveness varies by chemical species. Polishing to remove trace quantities of organic from the treated water is done by activated carbon filtration. Dissolved solids removal is accomplished in an ion exchanger. Aqueous liquids with high concentrations of dissolved organics are subjected to distillation to remove the organics. The distilled organics are sent to the organic destruction subsystem and the aqueous stream with low organic concentration is treated as outlined above.

For liquids with low levels of TDS the same treatments as discussed for high TOC waste are appropriate, with the exception of gross organic removal. Again, waste characterization will dictate which unit operations are necessary for each batch tank.

For liquids with high levels of TDS or contaminated with metals, the treatment train involves precipitation and settling steps for TDS and metal removal. Neutralization with appropriate chemicals such as sodium hydroxide is performed in an agitated tank. Depending on the waste characterization, metals precipitation from the waste followed by settling is performed to convert the dissolved and particulate metals to insoluble salts. The sludge is collected in a sludge settling tank and the clarified aqueous layer is transferred to suspended solids removal and ion exchange equipment. Ion exchange vessels are supplied with 
mercury selective resins. Further polishing is available by filtration with sulfur impregnated carbon filters. The sludge is concentrated in an evaporator and sent to the polymer stabilization subsystem.

In addition to the primary treatment trains, each waste type can be routed to, or can bypass, a specific treatment. The aqueous waste treatment subsystem concentrates all sludge waste produced by the various treatment trains prior to stabilization. Spent ion exchange resin from treatment processes is dewatered and sent to the organic destruction subsystem.

The unit operations remove radionuclides and RCRA-regulated metal, and to reduce trace organic concentrations to regulatory discharge levels, thus allowing the treated water to be either recycled or discharged.

\subsubsection{Catalytic Wet Oxidation Organic Destruction Subsystem}

Figure 2-8 presents the PFD for the organic destruction subsystem. Figure 2-9 presents the subsystem's perspective view. The organic destruction subsystem is based on a catalytic wet oxidation process which uses an oxidant in a catalyzed solution to enhance the destruction of organic wastes. The waste feed is shredded and continuously added to the reaction vessel through a solid or liquid-feed assembly. Because organic destruction involves exothermic reactions, the waste is metered into the reactor to prevent a runaway reaction. Active cooling is provided for temperature control.

The catalytic wet oxidation process is a method of catalytically oxidizing waste organic compounds to destruction using an iron oxidant which is regenerated by oxygen. Iron or Fe(III) in a hydrochloric acid solution oxidizes organics to carbon dioxide $\left(\mathrm{CO}_{2}\right)$ and hydrogen ion $\left(\mathrm{H}^{+}\right)$. The iron is reduced during this process to $\mathrm{Fe}(\mathrm{II})$. Oxygen $\left(\mathrm{O}_{2}\right)$ is added to oxidize the $\mathrm{Fe}$ (II) back to $\mathrm{Fe}$ (III). The oxygen also reacts with the $\mathrm{H}^{+}$to produce $\mathrm{H}_{2} \mathrm{O}$. A set of homogenous co-catalysts influences these reactions. Typically, these co-catalysts are copper ions and one or more of the ions platinum(IV), palladium(II), and ruthenium(III). Mercury, radioactive metals, and heavy metals are dissolved and concentrated in the acidic catalyst solution.

The process conditions are expected to be pressures of 20 pounds per square inch gauge (psig) and temperatures of between $300^{\circ} \mathrm{F}$ and $400^{\circ} \mathrm{F}\left(150^{\circ} \mathrm{C}\right.$ to $\left.200^{\circ} \mathrm{C}\right)$, depending on the waste form and composition. The 1000 gallon reactor can be operated at pressures of up to 200 psig. The standard solution consists of 60 percent by weight (wt percent) ferric chloride, approximately $3.6 \mathrm{wt}$ percent hydrogen chloride $(\mathrm{HCl})$, with the remainder being water. Catalyst concentration is approximately $0.001 \mathrm{M}$ (the number of moles of catalyst ion in 1 liter of solution). The reactor solution is agitated by recirculated headspace gases and by a mechanical agitator.

Oxidation of organics occurs principally at the surface of contact between the organics and the reagent solution. Carbon dioxide generated by the oxidation reaction is removed from the oxidation vessel with the headspace gases, which are primarily water vapor, $\mathrm{CO}_{2}, \mathrm{O}_{2}$ and $\mathrm{HCl}$. Offgas is passed through a condenser to remove most of the water vapor and $\mathrm{HCl}$. A portion of the condensed acidic water is recycled to the reagent storage and feed tank. The remainder of the acid is neutralized using caustic. The neutralized salt solution is routed to the aqueous waste treatment subsystem for further treatment.

As the inorganic residues build up in the reaction vessel, a side stream of reactor solution is removed from the bottom of the vessel and passed through a filter to remove residual solids. The acid filtrate is recycled to the reactor. Solids collected at the filter are rinsed in a wash tank with a mild hydrochloric acid solution, and sent to the polymer stabilization subsystem.

Periodically, the concentration of dissolved metals and radionuclides build up to a level that affects the acid concentration. When this occurs, the feed to the reactor is stopped, organics are allowed to react until 
gone, and the reactor solution is cooled. In the precipitator, the solution is neutralized and the metals are precipitated from the reactor solution as hydroxides or oxides by the addition of caustic. Following filtration, the filtrate salt solution is transferred to the aqueous waste treatment subsystem and the metal precipitates are routed to the polymer stabilization subsystem.

\subsubsection{Process Residue and Inorganic Sludge Treatment Subsystem}

Figure 2-10 presents the overall PFD for the process residue and inorganic sludge treatment subsystem. Figure 2-11 presents the subsystem's perspective view. This subsystem employs an indirectly heated vacuum desorption dryer to remove volatile organics and volatile inorganic compounds from the process residue and inorganic sludge prior to leaching/washing operations for mercury removal.

The waste sent to the process residue treatment subsystem has been size reduced to less than one-inch particles in the receiving and preparation subsystem. The vacuum desorption unit, which is operated in the batch mode, separates organic contaminants from inert solids under low pressure at moderate temperatures. Volatile organics and mercury are condensed and recovered as a liquid.

The waste is fed into the vacuum desorber in batches through a vacuum valve at the top of the desorption unit. In the first step of the process, the waste stream is charged through an inlet flange located on top of the jacketed vacuum desorption vessel. The desorption vessel is sealed and nitrogen is used to purge the desorption vessel and gas equipment of air, removing the potential for combustion of waste material or desorbed gas. The purge stream also assists in the desorption of contaminated vapors.

The second step involves slowly heating the nitrogen to desorb the organic components from the waste. The vapors exit the vessel by passing through a chamber equipped with a filter for removal of entrained particulate. Particulate is subsequently returned to the desorption unit and is removed with the processed waste solids.

The waste is also heated indirectly using a hot oil heating unit. The hot oil is circulated through a baffled external jacket which surrounds the desorption vessel. For homogenous feed such as soil or sludge, a top mounted agitator is used to stir the waste. The agitator shaft and impeller contain annular spaces into which the hot oil can be circulated. Heat is circulated through the waste for one to two hours increasing the waste temperatures to $500^{\circ} \mathrm{F}$. As the waste is heated, vacuum (as low as $25 \mathrm{~mm}$ mercury [Hg]) is applied for periods of from 15 minutes to several hours, depending on the feed. When treatment is complete the vacuum is released, the unit is opened, and the treated material is discharged to a transportable hopper at the bottom of the desorption vessel.

The bottom solids removed from the vacuum dryer are collected and processed for mercury removal by leaching. The solids are first washed with a potassium iodide and iodine leaching solution in an agitated mixing tank and then rinsed with fresh water. In Solid Wash Stage 2, the solids are washed with a hydrogen peroxide and sulfuric acid solution. The wet solids are filtered, collected in hoppers, and routed to the polymer stabilization subsystem.

The leaching solution and subsequent rinse water are collected and passed through a column containing steel wool which removes mercury. In the column, the mercury is precipitated from solution and captured by the steel wool. The mercury contaminated steel wool is routed to mercury amalgamation. The mercury amalgamation process is sufficiently adaptable to handle this waste. The remaining solution is processed further in a metal precipitation/settling tank to remove dissolved metals. Metals and settled solids are sent to polymer stabilization. The aqueous phase from the precipitation is treated to recover the iodine by the use of a hydrogen peroxide and sulfuric acid mixture. The recovered iodine and the iodide solutions are recycled to the leaching tank while the spent acid and peroxide are recycled to the final acid rinse tank. 
Desorber offgas passes through three condensers connected in series. The primary cooler/condenser reduces temperature to ambient conditions. Condensate, which may include aqueous mercury solution, elemental mercury, as well as some organics, is collected and allowed to settle. The condensed aqueous mercury solution, is routed to the aqueous waste treatment subsystem and any organic phase is sent to organic destruction subsystem. The dense lower elemental mercury layer is sent to the mercury amalgamation subsystem.

In the second condenser chilled water reduces the offgas temperature to near $45^{\circ} \mathrm{F}$. In the third condenser chilled brine further reduces the offgas temperature to near $0^{\circ} \mathrm{F}$ for effective condensation of organics and water. The condensed organic liquid is captured and sent to the organic destruction subsystem. The remaining offgas from the dryer is routed to the air pollution control subsystem.

\subsubsection{Bulk Soil Treatment Subsystem}

Figure 2-12 presents the PFD for the Bulk Soil Treatment subsystem. Figure 2-13 presents the subsystem's perspective view. The bulk soil treatment subsystem uses aqueous wash technology which employs water and surfactants for contaminant removal, with subsequent recycling of the water and surfactants. The aqueous wash process of the bulk soil treatment subsystem is composed of :

- feed preparation equipment

- a surfactant/wash water tank

- $\quad$ an agitated wash tank

- vibrating screen and hydrocyclone separators

- a solid/liquid rinse contractor vessel

- a solubility reduction process to separate surfactant/contaminant from wash water

- ultrafiltration

- soluble metals removal by precipitation followed by dissolved air flotation (DAF)

- surfactant/contaminant separation

- a surfactant preparation tank.

The process is operated in a continuous mode. Costs and design are based on a one shift operation, to minimize operations costs.

Several unit operations that utilize conventional equipment are required. Recycling occurs within most unit operations, minimizing the need for makeup water or surfactant, and reducing the final output volume. The wastes are first processed in an attrition mill to size reduce the waste stream. Wastes are transferred to an agitated wash tank for primary organic removal. A wash water solution containing surfactants and other additives is continuously fed to the wash tank along with the wastes. A 45 degree pitch turbine agitator stirs the tank contents. For soil treatment, four tons of wash solution are used for each ton of soil. The water in the wash tank is maintained between $140^{\circ} \mathrm{F}$ to $176^{\circ} \mathrm{F}\left(60^{\circ} \mathrm{C}\right.$ to $\left.80^{\circ} \mathrm{C}\right)$.

Organics and metals will be removed from the soil or residue by the surfactants, additives, and agitation. A slurry of soil and wash water are continuously removed from the bottom of the wash tank and sent to the two step solid/liquid separation equipment. Vapors from the wash tank are vented to two heat exchangers in series. Air is released and the small volume of collected liquids, volatiles and water are sent to the solubility reduction separator discussed below.

The wash tank discharge passes through a two step solid/liquid separation system. The stream is passed over a rotating screen which filters out solids. The filtrate is sent through one or more small bore hydrocyclones for further solids separation. The solids from the screening process, which include the 
contaminant, surfactants and water, are sent to a countercurrent soilliquid contactor. The filtrate is sent to the solubility reduction separator.

Rinse water is added to the rinse contactor at a rate of six tons of water to one ton of soil. The contactor is a trough-like vessel with paddles in which the soil is rinsed clean of the contaminants and surfactant. Following this step, the treated waste is dried and transferred to stabilization. Soil is vitrified while process residue is stabilized with polymer. Both the contaminated contactor rinse water and filtrate from the solid/liquid separation are sent to the solubility reduction separator described below.

The solubility reduction separator uses an approach that is proprietary to the vendor. In this step, temperature and $\mathrm{pH}$ effects are used to alter the solubility of the surfactant dramatically. The surfactant forms a dense lower phase, usually containing the contaminant. Colloidal matter such as humic components of soil also collect in the surfactant layer. The surfactant can be described as an adsorption agent, a "liquid active carbon", which holds the contaminant. The top layer of liquid, about 40 percent of the wash/rinse liquid, is recycled to the wash water storage tank. The remaining water layer is sent to ultrafiltration. The surfactant layer, containing the contaminant, is sent to a splitter process to separate the surfactant for reuse.

Ultrafiltration, also referred to as inertial separation, separates the incoming liquid into two liquid streams: the first containing a high level of particulate, and the second, the filtrate containing essentially no particulate. The liquid feed is pumped through parallel porous metal tubes manifolded together. The feed stream, stored in a tank, is continuously recirculated through the ultrafiltration tubes at high speed. The principle of ultrafiltration utilizes inertia whereby a particulate in a stream moving quickly through a porous metal tube remains with the stream while clear water passes through the porous metal. Thus, the filtrate is free of particulate and the particulate stream becomes more concentrated. Periodically, a back pulse of pressurized air is injected into the clear side of the tubes to remove particulate that may clog the tube pores. The concentrate from ultrafiltration is returned to the solubility reduction stage. The filtrate is transferred to a rinse water storage tank at the solid/liquid contactor for reuse. The concentrated particulate can also be removed as a sludge and sent directly to polymer stabilization.

When soluble metals are present, they may remain with the rinse water following ultrafiltration. A process similar to DAF is used to precipitate the metals in a froth. The froth is skimmed off the top of the flotation unit. The collected waste is sent to the polymer stabilization subsystem.

The separation of the surfactant and contaminant "complex" is performed in the splitter. Surfactant is separated by a proprietary process which includes steam stripping, followed by solvent extraction. The solvent is distilled and recycled. The extracted surfactant is recycled to the wash tank. Condensed organics from the stripping and nonvolatiles from the extraction process are sampled and sent to an appropriate subsystem for further treatment. If the waste is highly organic, it will be sent to organic destruction. If the waste contains high levels of mercury or other metals, it will be transferred to aqueous waste treatment.

Although this is a wash process, the only output to aqueous waste treatment is during shutdown or cleanup. All water, except that which exits with the wet treated waste, the metal froth or the organic contaminants, is recycled.

\subsubsection{Debris Treatment Subsystem}

The soft debris, and the open debris waste streams are subjected to treatment prior to vitrification. The complex debris waste stream is separated into metals and mercury contaminated debris.

2.1.7.1 Soft Debris Treatment Subsystem by Catalytic Wet Oxidation. Figure 2-14 presents the PFD for the soft debris treatment subsystem. Figure 2-15 presents the subsystem's perspective 
view. Soft debris is received for treatment from the receiving and preparation subsystem. The debris is passed through a tertiary shredder to reduce the debris to pieces that are less than a half-inch in size. The debris is then sent to a CWO subsystem where it is fed into a stirred reactor containing the same acid solution used for organic liquid destruction. A slower reaction rate is expected for soft debris compared to organic liquids. A larger vessel (2500 gallons) with a longer residence time is required. The soft debris reacts with the hot acid medium to form water vapor, $\mathrm{CO}_{2}, \mathrm{O}_{2}$, and $\mathrm{HCl}$. Most inorganic components in the soft debris are converted to insoluble salts which are removed from the acid mixture by the filtration stabilization subsystem.

2.1.7.2 Complex Debris Treatment. The complex metal debris is sent to a metal melter. The mercury contaminated debris is sent to the mercury amalgamation subsystem where it is retorted to remove the mercury prior to vitrification. The remaining non-metallic complex debris is vitrified. The vitrification subsystem is described in Section 2.1.14 below. The metal melter is described in Section 2.1.15 below.

2.1.7.3 Open Debris Treatment by High Pressure Wash. Figure 2-16 presents the PFD for the open debris treatment subsystem. Figure 2-17 presents the subsystem's perspective view. This subsystem collects and treats open debris whose internal and external surfaces are contaminated with organics and heavy metals which can be removed using surfactants delivered by a high pressure spray wash. The debris treatment subsystem is composed of a shredder, a spray/rinse chamber, a particulate filter, and a surfactant preparation tank. The subsystem is operated in batch mode.

The feed material is placed inside a 12 feet long, 7 feet wide, 8 feet deep spray/rinse chamber using a crane. Monolithic pieces (11 feet long, 7 feet wide, 7 feet high) and 55-gallon drums are placed in the chamber (Dosani, 1995). Smaller loose pieces with a minimum size of 1-inch in any dimension are placed in 4-feet diameter by 4-feet high metal baskets which are lowered into the chamber by crane. The size of the basket openings determines the size range of the loose pieces. Each basket weighs 1,200 pounds and is capable of containing 1 to 2 tons of debris. The debris is placed in the cleaning chamber which is closed to prevent the release of fugitive volatile emissions (Taylor, 1994).

Three stages of high pressure wash occur in the cleaning chamber. In the first stage, the chamber is flooded with approximately 2,000 gallons of $160^{\circ} \mathrm{F}$ surfactant solution from the detergent holding tank. The surfactant is industrial grade and biodegradable. The optimum cleaning solution concentration is 5 percent. The optimum solution temperature is $140^{\circ} \mathrm{F}$ (EPA, August 1991).

A $600 \mathrm{gpm}$ pump coupled with an educator creates the turbulence for this first stage of washing. Baskets that hold the debris are rotated in the turbulent solution. Monolithic pieces are simply immersed in the turbulent solution. This cycle loosens surface contaminants and provides some superficial cleaning. After this first wash stage, the surfactant solution is pumped from the cleaning chamber into the detergent holding tank.

In the second wash stage, which achieves most of the cleaning, the detergent solution is delivered at a rate of $500 \mathrm{gpm}$ through spray nozzles generating $100 \mathrm{psi}$ of pressure. Nozzles are located inside the basket and throughout the chamber sides and bottom, and the inner surface of the cover. The surfactant solution returns to the detergent holding tank after passing through an oil/water separator to remove oil and solids. The water separator in the aqueous waste treatment system is used for this purpose. Reconditioning the surfactant solution reduces the volume of water consumed in this subsystem.

In the third wash stage, clean water is applied through the spray nozzles to remove residual contaminated liquid from the surfaces of the debris. The washed, non-metallic debris is then sent to the vitrification stabilization subsystem for further processing. 
Oil, solids, and paper solubilized in the wash are collected in the oil/water separator and then sent to the organic destruction subsystem for further processing. Excess rinse water and the spent surfactant solution are transferred to the aqueous waste treatment subsystem. The treated water from the aqueous waste treatment subsystem is recycled back to the water holding tank.

\subsubsection{Metal Melting Subsystem}

Figure 2-18 presents the PFD for the metal melting subsystem. Figure 2-19 presents the subsystem's perspective view. Metals that cannot be decontaminated by mechanical means (surface blasting, grinding, etc.) are sent to this subsystem. These are primarily complex metal items such as pumps, valves, etc, and also drums that have deteriorated such that they cannot be decontaminated. Major functions provided by this subsystem include size reduction function, and metal melting function. Size reduction is accomplished by cutting or by disassembly of large metallic waste items.

The metal melter is an induction type melter. The melting process is designed to separate the molten product into a metal and a non-metal (slag) streams. Most radioactive material is removed in the molten slag, which is containerized and sent to the vitrification process for stabilization and subsequent disposal. Clean molten metal is poured into an ingot, cooled, and recycled within the DOE complex. Off-gas from the induction melter is sent to the APC subsystem for treatment.

\subsubsection{Metal Decontamination Subsystem.}

Figure 2-20 presents the PFD for the metal decontamination subsystem. Figure 2-21 presents the subsystem's perspective view. The decontamination subsystem has size reduction tools (cutting torch, saw and shears) and an inspection assay area. Decontamination is accomplished by using grit blasting and high pressure wash sprays within blasting booths designed to entrain blasting material and contaminants. Decontaminated metal is assayed, and approved metals are recycled within the DOE complex. Contaminated liquid is separated from the grit and recycled within the subsystem. Excess liquid is sent to the aqueous treatment subsystem. Contaminated grit is sent to the process residue treatment subsystem. Fugitive dust is captured in a prefilter and high-efficiency particulate air (HEPA) filters.

\subsubsection{Lead Recovery Subsystem.}

Figure 2-22 presents the PFD for the lead recovery subsystem. Figure 2-23 presents the subsystem's perspective view. This subsystem has a decontamination train and an electrically heated roasting oven. The decontamination train has mechanical devices, including saws, shears, and sanders, to cut and remove metal cladding from lead. Decontamination of lead takes place in scarring and abrasive blasting booths where a liquid abrasive solution is used to remove a thin layer of contaminant. Lead which is decontaminated can be recycled within the DOE complex. Drums containing cleaned material are washed and transferred to the certification and shipping subsystem. Lead that cannot be decontaminated due to entrained contamination (gloves, aprons, etc.) is sent to the melter equipment where the lead is melted and cast. The oven is used to melt lead that is not suited for decontamination by mechanical means. The oven includes dry gas filtration for particulate removal from the offgas, prior to the offgas being transferred to the APC subsystem.

\subsubsection{Mercury Amalgamation Subsystem.}

Figure 2-22 presents the PFD for the mercury amalgamation subsystem. Figure 2-24 presents the subsystem's perspective view. This subsystem uses a retort, followed by a condenser, to reclaim mercury from contaminated solids. After mercury removal, the solids are sent to the primary stabilization subsystem. Offgas from the mercury condenser is treated in a secondary combustion chamber followed by a wet-dry APC. 
Recovered elemental mercury is either recycled or amalgamated with zinc or copper in an amalgamation reactor. The mercury amalgamation reactor treats elemental mercury and any steel wool impregnated with mercury from the process residue treatment subsystem leaching process. The mercury is transferred to an amalgamation operation where the mercury is combined with copper (or zinc) powder, steel shot (for proper mixing), and nitric acid. This combination is mixed to form a copper-mercury amalgam, eliminating free mercury. The amalgam is packaged for assay and inspection to ensure that it meets TCLP standards.

\subsubsection{Special Waste Treatment Subsystem}

It is assumed that there are special wastes that will require capabilities not included in the basic system design concept. The treatment subsystem for special waste is located in a room equipped with a crane and all utilities needed for installing treatment equipment to process special wastes. Special treatment equipment will be identified and provided on a case-by-case basis during facility operation. The cost estimate for all ENTS systems includes the space in the building, but not processing equipment. Equipment and operation costs have not been estimated for special treatment processes.

\subsubsection{Polymer Stabilization Subsystem}

Figure 2-25 presents the PFD for the polymer stabilization subsystem. Figure 2-26 presents the subsystem's perspective view. The polymer stabilization subsystem receives (1) salt waste from the receiving and preparation subsystem, (2) salts, fines and concentrated scrubber liquid waste from aqueous waste treatment and the air pollution control subsystems, (3) treated process residue solids and desorbed solids from the process residue and inorganic sludge treatment subsystem, and (4) sludges from the soil and debris washing subsystems.

Stabilization of wastes involves polymer encapsulation using polyethylene. The subsystem has a dryer which removes water from the incoming waste. A tertiary shredder is available to reduce the waste size prior to an extrusion operation. The dried, shredded waste and polymer are metered into a mixer or extruder which heats and mixes the polymer with the waste at a ratio of one part polymer to one part waste by weight. The extruder feeds the mixture directly into a drum.

When the filling operation is complete, the drum is automatically capped and sent to a wiping and decontamination station. If surface contamination is detected, the container is washed by high-pressure water jets or blasts of dry ice. The inspected container is sent for assay, certification, and shipment to storage or disposal facilities.

\subsubsection{Air Pollution Control (APC) Subsystem}

Figure 2-27 presents the PFD for the air pollution control subsystem. Figure 2-28 presents the subsystem's perspective view. The APC subsystem receives offgas from other subsystems that produce offgas requiring treatment. This offgas may include gaseous reaction products from organic destruction (mainly carbon dioxide), desorber offgas, aqueous waste treatment offgas, and offgas from washing operations. Several unit operations are provided in this subsystem.

The APC subsystem is composed of (1) a metal filter, (2) pre-filter, (3) GPCR unit, (4) a wet gas scrubber, (5) mist eliminator, (6) activated carbon for trace organics removal, (7) sulfur impregnated activated carbon for mercury vapor removal, and (8) a system for continuous emissions monitoring (CEM). Treatment occurs near room temperature and pressure. 
Gross particulate is removed from the gas stream by a metal filter. The gas is also passed through a HEPA filter to remove fine gas particulate. Once the particulate is removed from the gas stream, the gas enters the GPCR. Unlike carbon adsorption, the gas delivered to the GPCR does not require any temperature reduction or moisture removal. The relative humidity of the inlet gas can be as low as 0 percent and as high as 90 percent. The GPCR is a cylindrical quartz vessel with an inner high voltage electrode and a grounded electrode on its outer surface. The annulus of the vessel is filled with dielectric pellets. A strong alternating electric field placed across the packed bed of dielectric particles creates a corona that causes the gas to partially ionize and to form a plasma in the voids between the particles. In the plasma, electrons are heated to extremely high temperatures while the bulk of the gas remains cool. The high-energy electrons in the plasma cause organic contaminant destruction through direct interaction with the contaminant molecules. Destruction also occurs through secondary ion and radical reactions.

The treated gas exits the GPCR and enters the acid gas scrubber. The gas scrubber includes a hydrosonic scrubber and a packed tower scrubber. The neutralized offgas passes through a mist eliminator. Final polishing is provided by granular activated carbon and sulfur impregnated activated carbon for mercury vapor removal. The treated offgas is monitored continuously before release to the atmosphere.

\subsubsection{Vitrification Subsystem}

Figure 2-29 presents the PFD for the vitrification subsystem. Figure 2-30 presents the subsystem's perspective view. In this subsystem, waste and soil or chemical additives such as $\mathrm{SiO}_{2}, \mathrm{Al}_{2} \mathrm{O}_{3}$ and $\mathrm{Fe}_{2} \mathrm{O}_{3}$ are metered into a mixer. The additives, which act as glass formers, are added in quantities proportional to the waste, which consists of treated soil and inorganic debris. The mixer combines the additives with the waste. From the mixer, the waste is discharged into a storage hopper. The mixture is metered from the hopper into a Joule heated melter. The melter vitrifies the waste and soil mixture by heating it to temperatures exceeding $2000^{\circ} \mathrm{F}$, and discharges the molten mass into a crucible.

The melter is equipped with a secondary combustion chamber (SCC). The vitrification process requires a minimal feed preparation. It uses air as the combustion gas in the SCC. This furnace can perform thermal treatment and vitrification. Solid residues from the melter, including radionuclides, are drawn off in two streamlines: a molten glass stream containing the vitrified waste, and a molten metal stream that contains residual metals contained in the pretreated waste. The molten product from the vitrifier is poured into crucibles placed in disposal containers. The containers are cooled, capped, and wipe tested. If surface contamination is found, the container are decontaminated by high-pressure water equipment. The inspected containers are sent for assay, certification, and shipment to storage or disposal facilities.

The APC subsystem is composed of dry and wet filtration equipment. The dry APC equipment includes quench cooling, baghouse filtration, charcoal filters, pre-filters, and a HEPA filtration system. The wet APC includes wet gas scrubbers, mist eliminator, and a $\mathrm{NO}_{x}$ abatement system. The APC system discharge is subject to continuous emissions monitoring (CEM).

\subsubsection{Certification and Shipping Subsystem}

Figure 2-31 presents the PFD for the certification and shipping subsystem. Figure 2-32 presents the subsystem's perspective view. In this subsystem, the physical and radiological properties of the packaged waste are characterized to allow certification in accordance with transportation, storage, and disposal requirements. Equipment utilized is similar to that in the receiving and preparation subsystem. The containers of packaged waste for shipment are weighed. An RTR unit examines the container to ensure that the matrix is homogeneous and does not contain free water. If TRU or alpha contaminated waste is processed, the TRU concentration is measured by a PAN assay unit. An SGS unit is used to assay the beta 
and gamma radioactivity. After inspection, the waste is either sent to a temporary storage area or loaded onto a truck for offsite or onsite shipment.

\subsubsection{Support Subsystem}

The support subsystem includes the facilities necessary to ensure continuous functioning of the treatment facility. These include (1) the auxiliary operating and maintenance galleries, (2) retrieval and transfer areas, (3) maintenance shop, (4) mechanical equipment room, (5) heating, ventilation and air conditioning (HVAC) equipment room, (6) motor control center (MCC), and (7) electrical rooms. Lighting, drainage, piping, fire protection, heating, and closed circuit television cameras are also provided as part of this subsystem. All of this equipment incorporates proven technologies. Costs for maintenance and equipment galleries are included for each treatment subsystem.

A main control room is provided for the control of process operations. The control room contains monitoring and control instrumentation, remote video monitors, communications devices and other equipment used to operate the facility. Costs for monitors are included in each treatment subsystem.

For maintenance needs, the treatment facility provides large corridors or galleries for equipment pullout and maintenance next to each cell. To minimize the consequences of equipment failure, the equipment layout is designed to allow the removal of equipment by crane. Equipment requiring repair is removed from its location and staged on a cart next to the maintenance gallery airlock. The equipment can then be transferred to the maintenance gallery. Repairs can be made in the maintenance gallery or, if necessary, removed to an offsite shop via the shipping or receiving subsystems. The gallery also has space to set up duplicate equipment outside the hot cell, and to assist in the remote repair of equipment using robotic or manipulator devices. Cell isolation minimizes the amount of process equipment that must be idled when a mechanical failure shuts down an operation.

The support subsystem includes the equipment to maintain three zones necessary for facility operation. One of them is a non-confinement zone. The zones are:

- Zone 1 - primary confinement. This zone encompasses process enclosures.

- Zone 2 - secondary confinement. This zone encompasses process areas immediately surrounding Zone 1 areas, including maintenance galleries.

- Zone 3 - non-confinement areas. This zone encompasses shipping, receiving, transfer corridors, control rooms, and administration and support areas.

Zone 1 confinement is achieved by drawing air into process enclosures through adjustable dampers and openings from Zone 2. Zone 2 confinement is achieved by drawing air from non-confinement areas in Zone 3. Zone 1 is maintained at negative pressure with respect to Zone 2 , while Zone 2 is maintained at negative pressure with respect to Zone 3 . Air drawn from Zones 1 and 2 is passed through air handling units comprised of prefilter, HEPA filter and charcoal filter banks. Zone 3 is serviced by an air supply unit equipped with temperature control and air filtration devices that are used to provide fresh conditioned air to the facility from outside.

The confinement system consists of dust collection, process ventilation, building ventilation, and HEPA filtration units. The combination of these units ensures confinement of airborne radioactive and chemical contaminants within the equipment and process area boundaries. The system removes airborne contaminants from the process areas and filters the air before exhaust. 
Dust control unit. Waste expected to generate dust will be handled within enclosures. Local vents and dust collection/filtration units are installed in areas where dust generation potential exists. After removal of dust, the filters exhaust the unit to the building ventilation and HEPA filter unit.

Process vent unit. Chemical vapors and fumes generated by processes are collected by a vapor collection system. Vented vapors are treated by filtration units designed to remove vapor, fumes and particulate before being exhausted to the vent system. Treated vapors are then sent to the building ventilation and HEPA filtration units prior to release to atmosphere.

Building ventilation and HEPA filtration system. This system ensures safe operating conditions by maintaining air flow from non-contaminated areas to areas of progressively higher potential contamination. A zoned approach is used, based on a cascade pressure differential principle.

The support subsystem includes the HVAC equipment. The functions of this equipment are (1) to provide heating and cooling requirements for the facility, (2) to provide air confinement barriers by removing air from the facility and recharging it with conditioned air from outside, (3) to remove airborne contaminants from the facility and to treat them prior to discharging treated and conditioned air to the atmosphere, and (4) to monitor radioactivity of treated air released to the atmosphere.

Ventilation air will be introduced into the building after passing through 85 percent efficient pre-filters and nuclear grade HEPA filters. Air will be exhausted from Zone 1 by industrial exhaust fans through a galvanized steel duct system. The exhaust fans will be located adjacent to the stack. Exhaust air will pass through the air treatment system. The air treatment system consists of a dust collection system, a fume abatement system, vapor filters, charcoal filters and nuclear grade HEPA filters. Treated air will be exhausted to the atmosphere through the stack. Intermediate sections will be provided with system redundancies to permit maintenance, testing or replacement of the abatement systems and filters.

The exhaust and makeup air systems operate continuously 24 hours a day, seven days a week. A redundant exhaust fan and filter train will be included to permit fan maintenance, filter change, and repairs.

Air volume measuring stations located in the makeup and exhaust ducting will continuously monitor the air flow rates and send signals to a digital controller. The controller will adjust the variable frequency drive of the exhaust fans to maintain a constant exhaust rate. This feature will automatically compensate for fluctuations in flow rates across the HVAC filters.

Atmospheric and room static pressure probes will send pressure measurement signals to the digital controller. The controller will adjust the variable frequency drive of the makeup air fan to control the volume of air entering processing area zones, and thus will maintain the negative to atmosphere pressure relationships required. A digital controller will be provided for all fans.

Air volume measuring stations will continuously monitor the exhaust air flow rate from each zone. A digital controller will adjust the position of a modulating damper located in the room exhaust ducts to maintain the desired air change rate for that zone. This feature will automatically compensate for pressure adjustment needs during events such as opening and closing doors of controlled spaces.

\subsection{System ENTS-2: Catalytic Wet Oxidation and Vitrification for Process Residues}

Figure 2-33 presents the overall PFD for System ENTS-2. Figure 2-34 presents the plan view layout. In this system vitrification replaces the grout stabilization used to treat waste streams in INTS System 5. In 
this system the vitrification subsystem rather than the vacuum desorption subsystem is used to treat the process residue and inorganic sludge.

The vitrification technology employed by this system is identical to the vitrification subsystem used in ENTS-1. The vitrification reactor employed by the ENTS-1 and ENTS-2 systems has a throughput capacity in excess of $3,000 \mathrm{lbs} / \mathrm{hr}$ for waste streams with less than $8000 \mathrm{Bta} / \mathrm{lb}$ heat content. All other subsystems of ENTS-2 are identical to the subsystems of ENTS-1.

\subsection{System ENTS-3: Catalytic Wet Oxidation and Polymer Stabilization}

Figure 2-35 presents the overall PFD of System ENTS-3. A plan view was not developed for the overall system because of its similarity to INTS System 5. In the ENTS-3 configuration the grout stabilization function of NT-5 is replaced by polymer stabilization which provides the only stabilization function for all waste streams, including all secondary waste streams generated by ENTS-3 subsystems. Figure 2-36 presents the polymer stabilization subsystem's perspective view. The polymer stabilization employed by ENTS-3 is capable of providing microencapsulation, and macroencapsulation stabilization functions. Extrudable waste such as process residue and dried sludge may be co-extruded in the polymer extruder. Co-extrusion is accomplished by feeding waste and Low Density Polyethylene (LDPE) simultaneously and co-extruding the product into waste disposal containers. Macroencapsulation is used for non-extrudable waste items which may qualify for treatment under the "EPA Debris Rule". Items such as metal debris are placed in disposal containers and LDPE is extruded through the polymer extruder and discharged into the disposal containers.

\subsection{System ENTS-4: Catalytic Wet Oxidation, Desorption, Compaction and Polymer Stabilization}

Figure 2-37 presents the overall PFD for System ENTS-4. Figure 2-38 presents the plan view layout. In the ENTS-4 configuration polymer stabilization provides the only stabilization function for all waste streams including all secondary waste streams generated by ENTS 4 subsystems. The polymer stabilization technology employed by ENTS-4 is a macroencapsulation process in which LDPE is extruded through a plastic extruder. The molten LDPE is discharged from the extruder into a container that holds compacted waste pellets. The waste pellets consist of process residue, soil, salts, secondary wastes, and size-reduced debris that is pretreated by screw compaction and sintering prior to polymer macroencapsulation. The sintering and polymer macroencapsulation process used as the basis for this system is the Claytec process.

\subsubsection{Claytec Subsystem}

Figure 2-39 presents the PFD for the Claytec subsystem. Figure 2-40 presents the subsystem's perspective view. Screw compactors with internal barrel pressures of 10,000 psi are used to compact waste and additives. The compaction force provides the bonding energy required to bind waste and the additives. Screw compactors are equipped with dies and cutters that are used to pelletize the waste. The pellets are then sintered at $2,200^{\circ} \mathrm{F}$ to form a dense crystalline ceramic structure with greater than $30,000 \mathrm{psi}$ strength.

Clay or other natural materials may be used as additives. The ratio of additive to waste is $1: 1$ by weight. Waste after screening and size reduction is added to pre-screened additives in a ribbon blender and is fed through a volumetric feeder to the screw compactor. The compacted waste is pelletized though a die cutter mechanism and is discharged into waste transfer bins. 
The waste transfer bins containing raw pellets are taken to the sintering oven. The sintering oven uses a shaker mechanism to transfer the compacted pellets through the oven heat zones and to minimize mechanical force experienced by pellets, thus minimizing breakage during sintering.

The sintering oven has three heat zones. Zone 1 allows the pellet temperatures to rise from ambient temperature to $2,200^{\circ} \mathrm{F}$. Zone 2 provides a residence time of one half hour at $2,200^{\circ} \mathrm{F}$ to allow for complete curing. Zone 3 provides gradual cooling to avoid cooling shock which might compromise the structural integrity of the sintered pellets. It is estimated that a pellet discharge temperature of $200^{\circ} \mathrm{F}$ is desirable.

The sintered pellets are discharged into half full 64 cubic feet containers which are cooled to ambient temperatures by forced air in the cool down chamber prior to transfer to the polymer macroencapsulation process.

During the polymer macroencapsulation process sintered pellet containers are placed inside the polymer fill chamber and filled with molted Low Density Polyethylene (LDPE). LDPE is the macroencapsulation medium. The macroencapsulated containers are then allowed to cure and cool in a forced draft cooling chamber prior to being capped and discharged from the subsystem.

\subsection{System ENTS-5: Catalytic Wet Oxidation with Increased Reaction Rates}

Figure 2-41 presents the overall PFD for System ENTS-5. Because this system is so similar to the INTS System 5, a perspective view was not developed. The aim of the ENTS-5 evaluation was to investigate the effects of processing rates on the life cycle cost of a facility. This system is identical to NT-5 except that the reaction rate through the catalytic wet oxidation process is assumed to be an order of magnitude (ten times) higher than the throughput for the CWO of INTS System 5. In performing this study it was assumed that the faster treatment rates would be accomplished by using a more advanced and more efficient version of the CWO process. It was further assumed that this process will maintain the processing logic and configurations of the CWO process identified in the INTS System 5. It should be noted that no such technology was actually identified, but preliminary results by the vendor indicate that faster reaction rates of two to three times may be possible. Also, application of external energy sources such as microwaves or ultrasonics may enhance the reaction rate.

The increased reaction rates of CWO in System ENTS-5 reduce the processing time for soft debris and organic waste. System ENTS-5 combines the two CWO subsystems into one, reducing the building space required. The reaction rate is so fast that only one shift of operation is necessary to process all the soft debris and organic waste. The reactor is kept to the size needed for treatment of only organics as depicted in the INTS System NT-5. However, a larger material handling system is now needed to prepare waste for treatment in the shorter time period. The APC subsystem is larger than that of System NT-5 to accommodate a higher gas flow when the CWO process is running. As discussed in Section 4.4, this system has an increase in equipment capacity and cost compared with INTS System 5. 


\subsection{Rationale for System Selection and Design}

The first two systems (ENTS-1 and ENTS-2) were chosen to identify the impact on cost and performance of using a hybrid nonthermal/thermal system by replacing grout stabilization with vitrification. In both systems, thermal processes were also used for metal treatment as follows:

- a metal melter for the complex, internally contaminated metal items

- a metal melter for lead waste with entrained contamination (e.g., lead gloves, aprons, etc.)

- a mercury retort and amalgamation for the mercury contaminated waste

The metal melting process increases the destruction of organic contaminants. The ingots produced from these metal melting operations are recycled instead of being stabilized in grout in both these ENTS systems. Thus, the amount of waste and total contaminants sent to disposal is decreased.

Replacing the mercury leaching process used in the nonthermal systems with a mercury retort increases the potential for removing mercury and mercury compounds from inorganic wastes. Mercury associated with organic wastes (including combustible debris) will be solubilized in the CWO solution. Subsequently, the mercury is precipitated as an insoluble sulfide and stabilized in polymer.

In ENTS-1, vitrification only replaces grout. Thus the only difference between NT-5 and ENTS-1 (except for the metal melters and mercury treatment identified above) is that treated debris and soils are vitrified in ENTS-1. As in NT-5, inorganic sludge and process residue are still treated in a vacuum thermal desorber and stabilized in polymer. This provides information on the cost and performance, and the processes required, if grout were replaced with vitrification.

ENTS-2 takes the concept one step further by eliminating the vacuum thermal desorber and treating the inorganic sludge in the vitrifier. Sludge is a difficult waste stream to treat. The efficacy of desorption or washing to remove organic contaminants from sludge is uncertain. Thus, ENTS-2 provides an enhanced waste form, improved system operational performance and more reliable destruction of organic contaminants in the sludge.

In both ENTS-1 and ENTS-2, polymer was used to stabilize (1) salt/sludge waste from aqueous waste treatment, (2) solids and fines from organic destruction, and (3) the primary salt waste. As in the thermal systems evaluated in the ITTS study and in the nonthermal systems evaluated in the INTS study, these waste streams were stabilized in polymer. All salts and sludges from the thermal APC quench and scrubber solutions were stabilized also in polymer. Thus, using polymer for stabilization of these waste streams in the ENTS study provides some continuity between systems for comparison. The primary reason for using polymer instead of vitrification for these waste streams is that many of these salts are chlorides that will volatilize in a vitrifier, end up in the offgas equipment, and will still need to be stabilized in polymer because they cannot be retained in a high temperature melter. Other salts, in particular sulfur based salts, affect electrical conductivity and may cause corrosion of electrodes and refractory. Vitrification of these secondary salt wastes is not a viable alternative.

In Systems ENTS-1 and ENTS-2 residue from CWO organic destruction is sent to polymer, and residue from CWO soft debris treatment is sent to vitrification. In System NT-5 residue from CWO organic destruction is sent to polymer, and residue from CWO soft debris treatment is sent to grout. Residue from organic destruction (organic liquids and sludges) consists primarily of salts from the spent CWO solution. The residue from CWO soft debris treatment consists of large amounts of alumina and silica (as per the mass balance on the debris elemental makeup in the INTS and ITTS reports) that can 
be vitrified or stabilized in grout. While this waste stream could be sent to polymer for stabilization, stabilization by vitrification was chosen to minimize disposal volumes.

ENTS-3 and ENTS-4 were evaluated to determine the impact on a nonthermal system of potentially improved waste forms. The stabilization methods used to achieve improved waste forms are:

- polymer stabilization only (ENTS-3)

- pelletizing and sintering the waste, then macroencapsulating it in polymer (ENTS-4)

As indicated previously, System NT-5 was used in these enhanced nonthermal systems for continuity so that we could compare the costs and performance of vitrification, polymer, and compacted pellets macroencapsulated in polymer against a baseline system using grout. System NT-5 was used as the basis for ENTS-5, again for continuity and comparison purposes, and because some reaction rate data were available to allow equipment sizing for an increased reaction rate and subsequent costing.

ENTS- 5 was evaluated to determine the benefits, if any, of pursuing R\&D to increase the reaction rate of nonthermal chemical oxidation processes. The initially perceived potential advantages of increasing the reaction rate are (1) an increased destruction efficiency if the same throughput and residence time are used as in the case of the slower reaction rate, and (2) decreased cost resulting from shorter operating time, fewer personnel, and smaller processing equipment if the residence time and equipment are scaled down.

If the same throughput and residence time are assumed to provide an increased destruction efficiency, then the personnel numbers or equipment required would not change from that used in a system with a slower reaction rate (e.g., System NT-5). In this case the life-cycle cost would be the same as that for NT-5, except for the additional capital and operating costs to achieve the higher reaction rate. The only option evaluated for System ENTS-5 was the decrease in the number of operating personnel and equipment to match the increased reaction rate. 


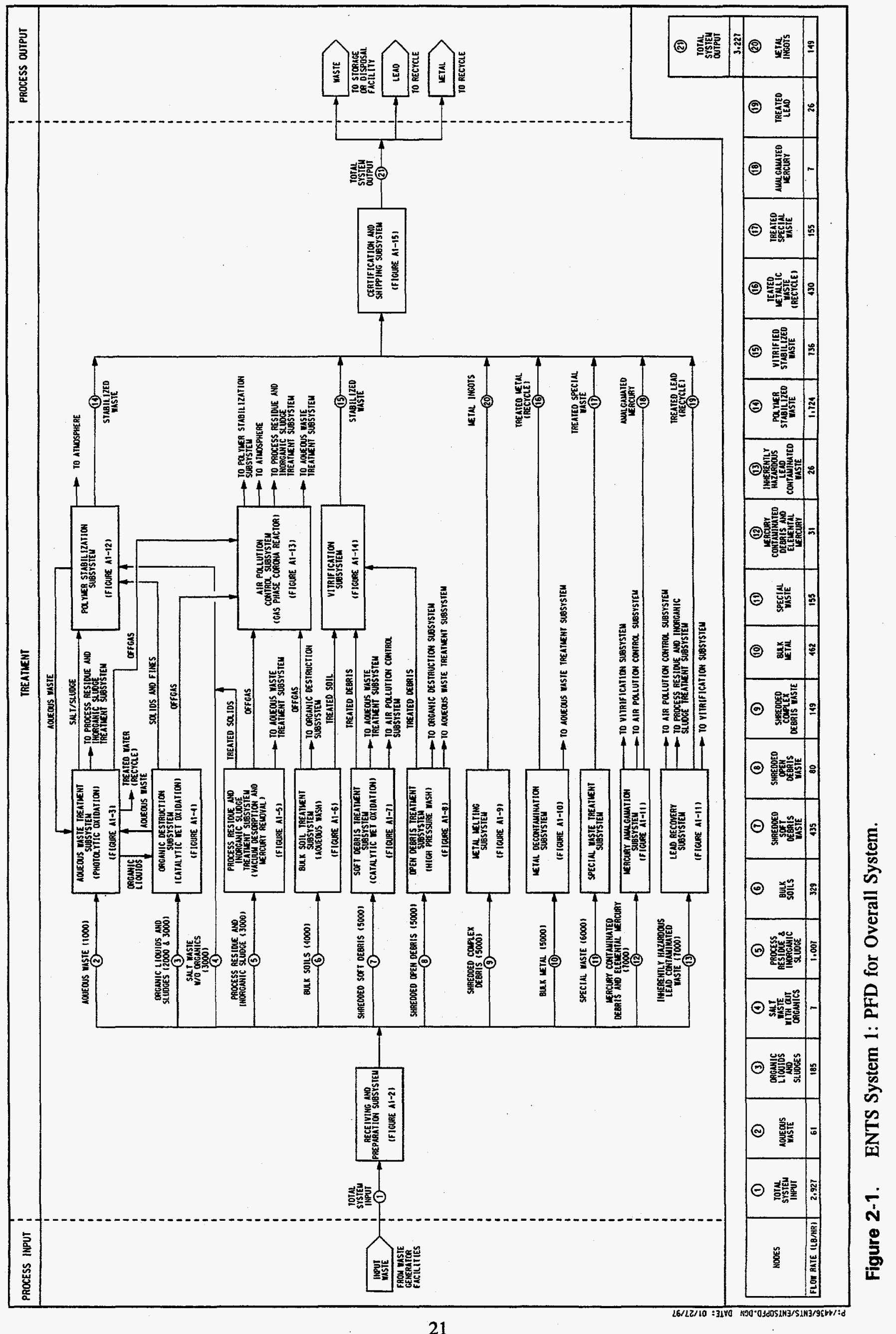




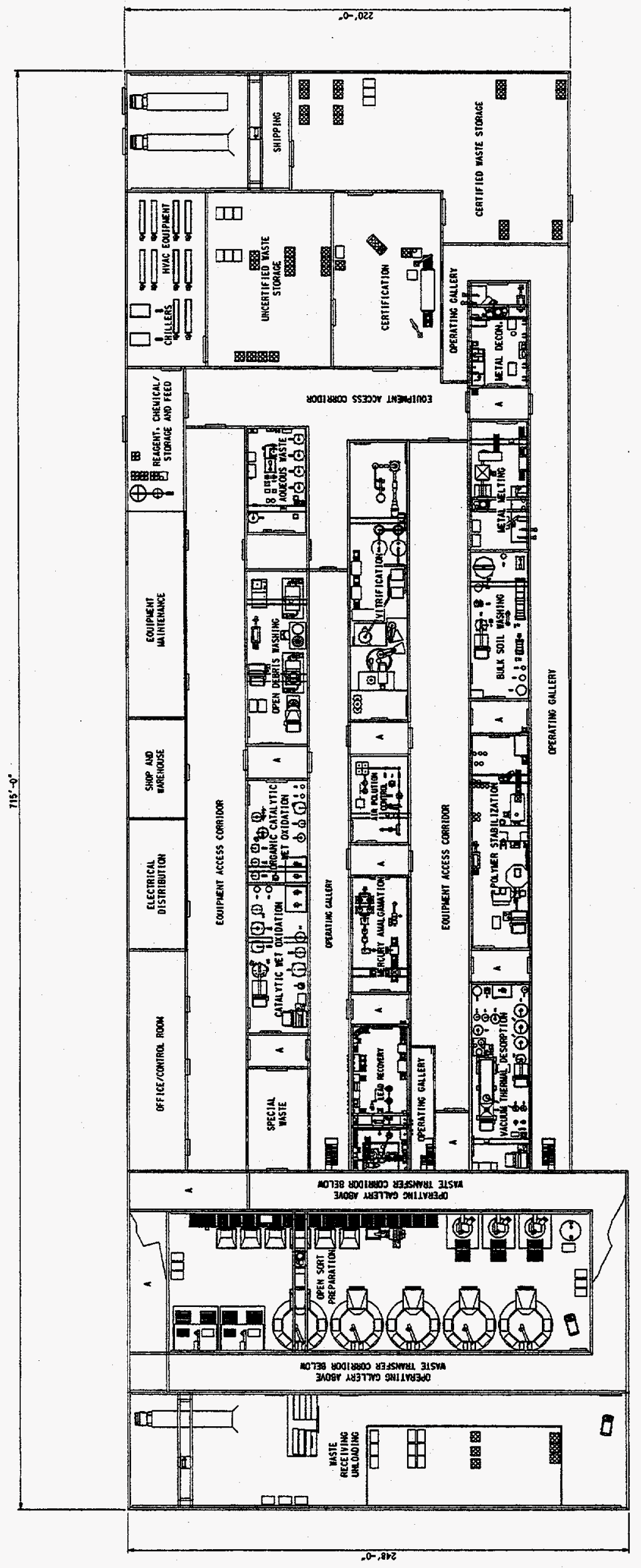

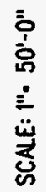




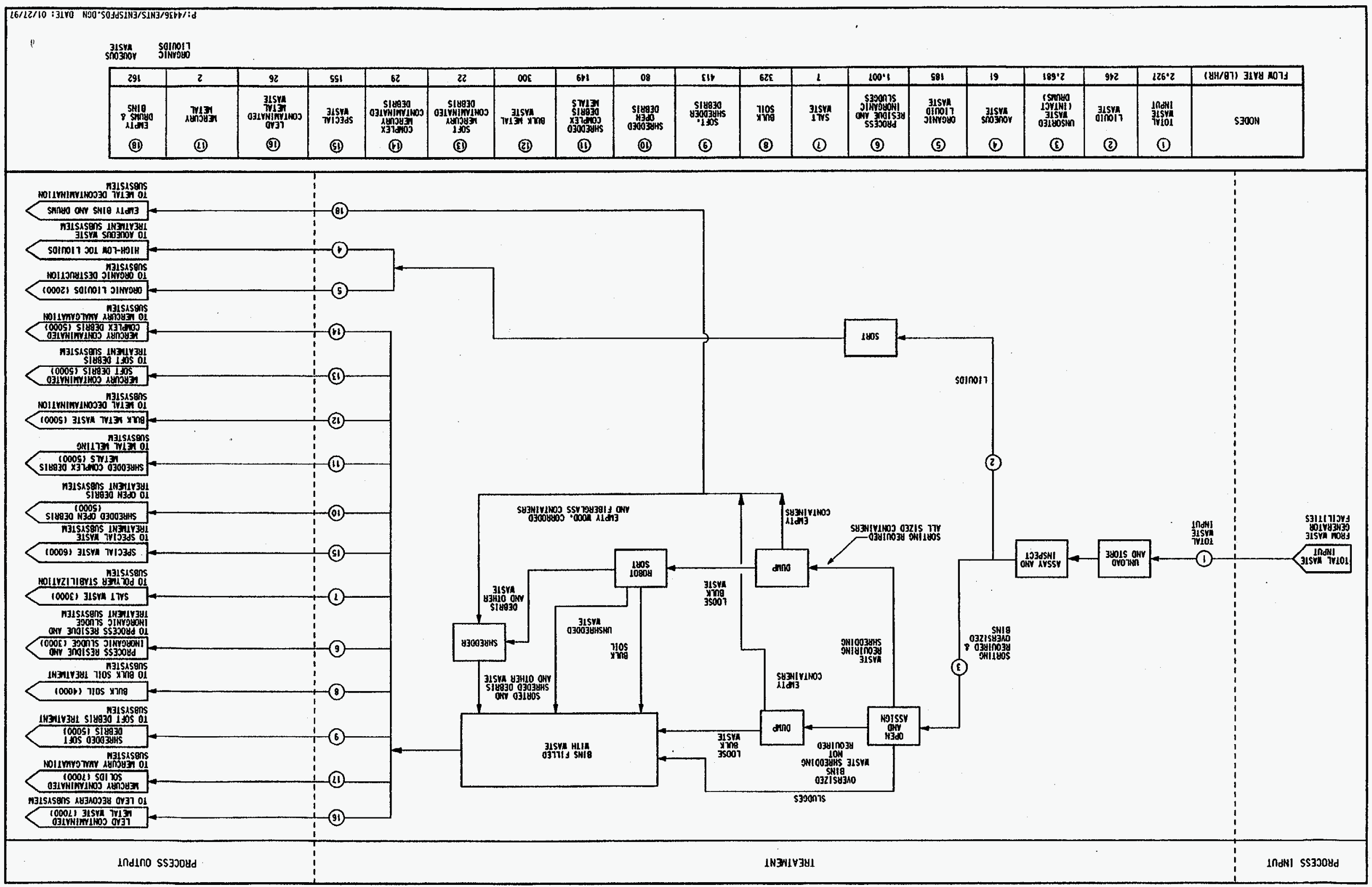




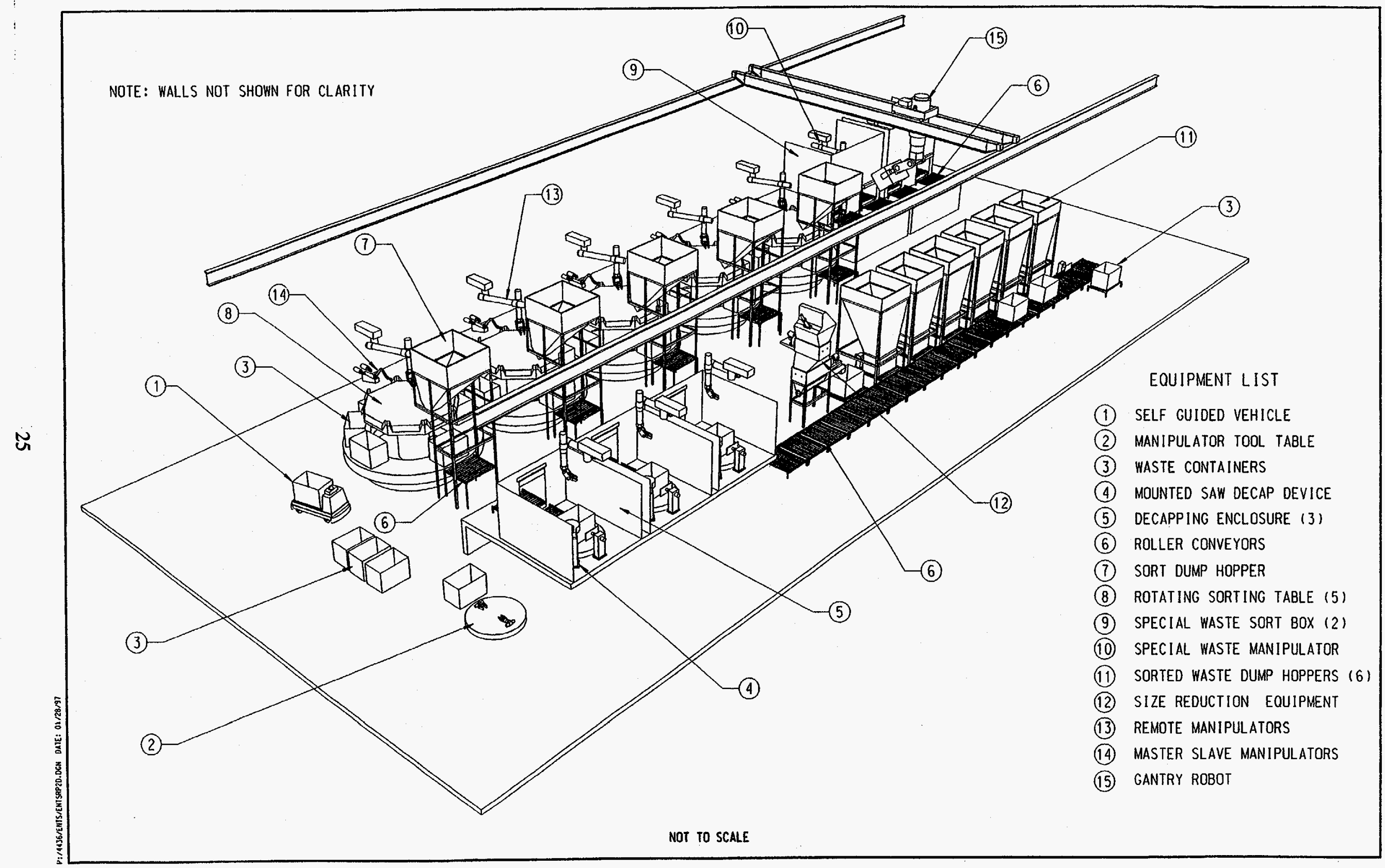

Figure 2-5. ENTS System 1: Perspective View of Decap, Sort, and Dump Cell of the Receiving and Preparation subsystem. 


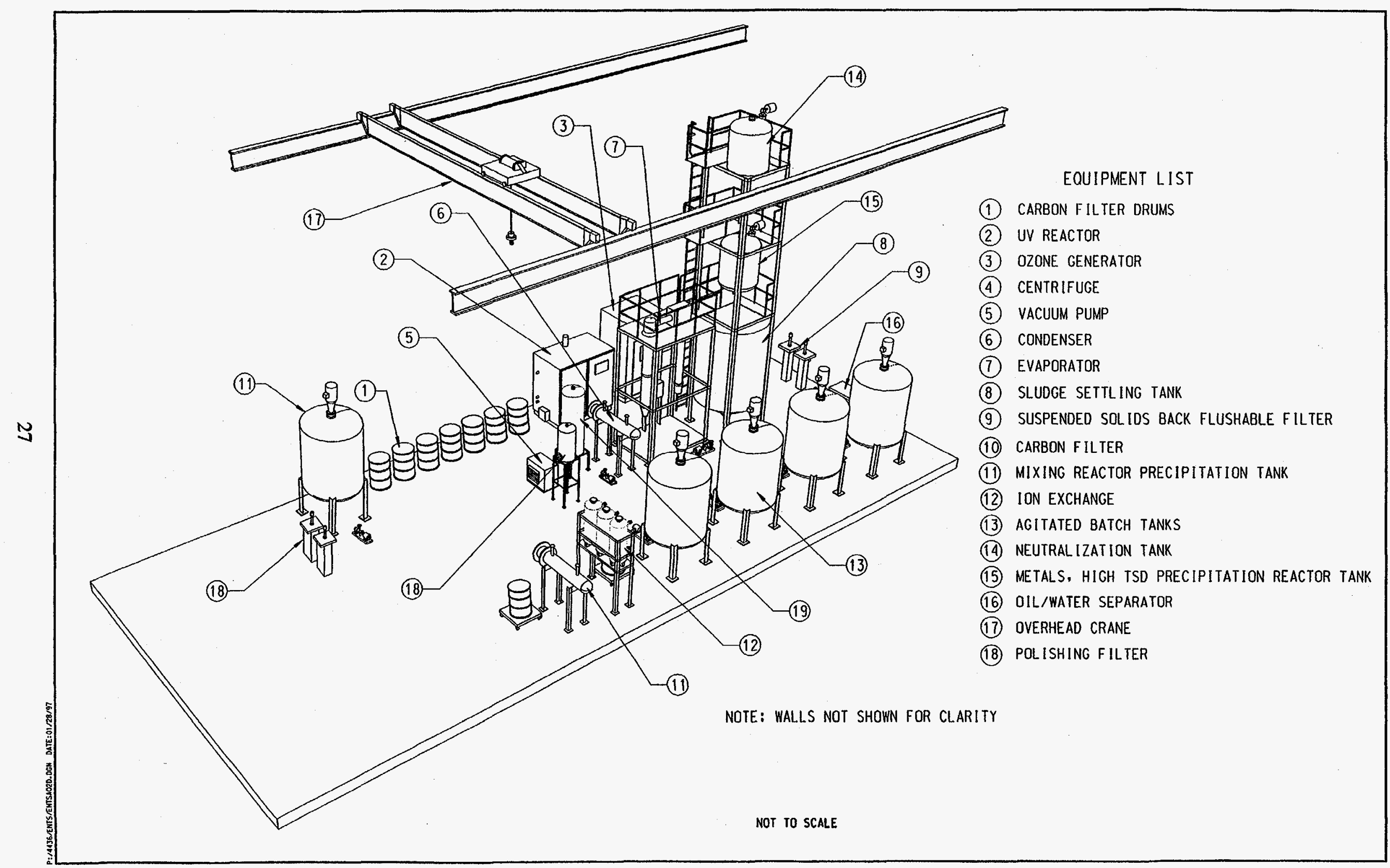

Figure 2-7. ENTS System 1: Perspective View of Aqueous Waste Treatment Subsystem. 


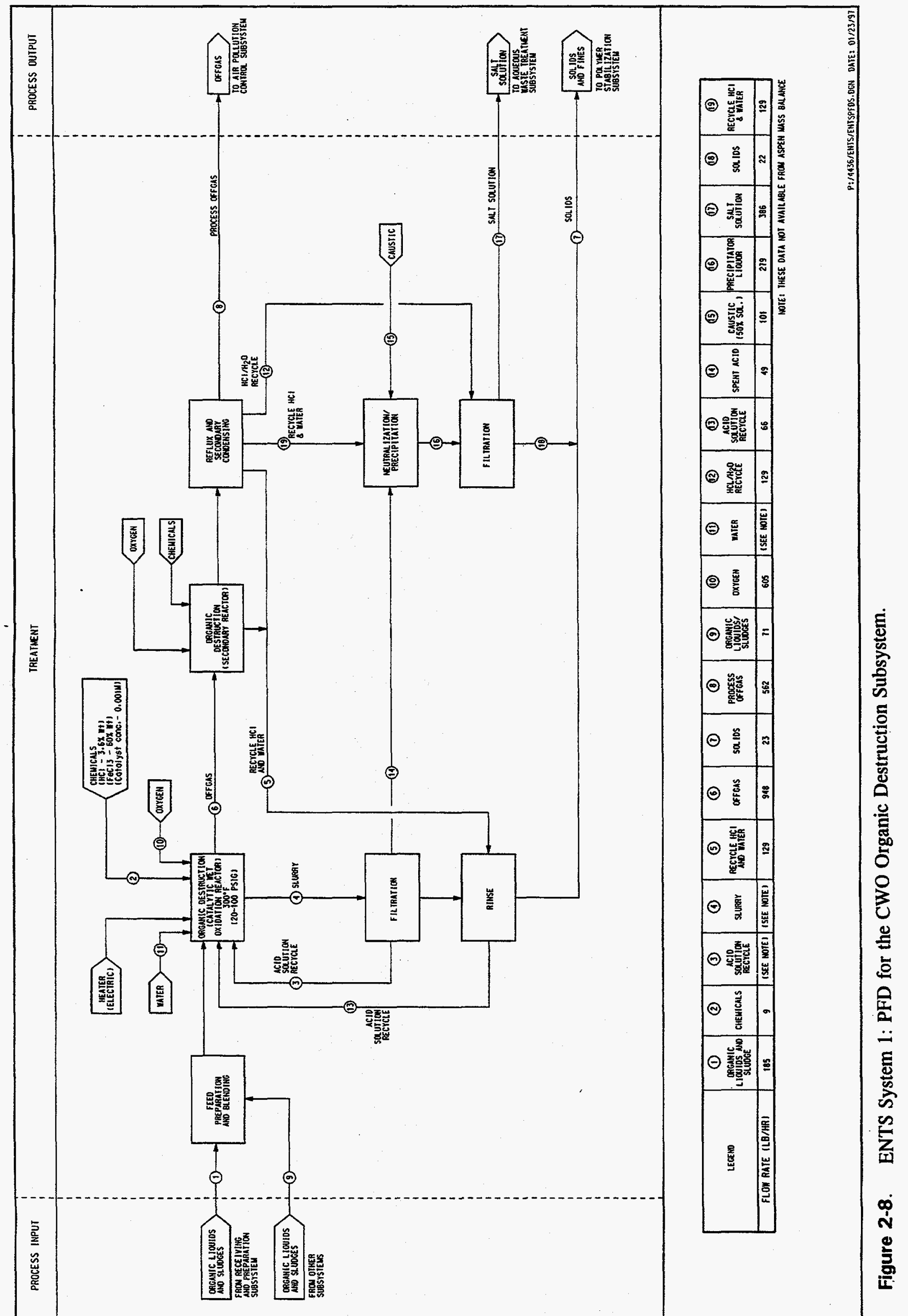




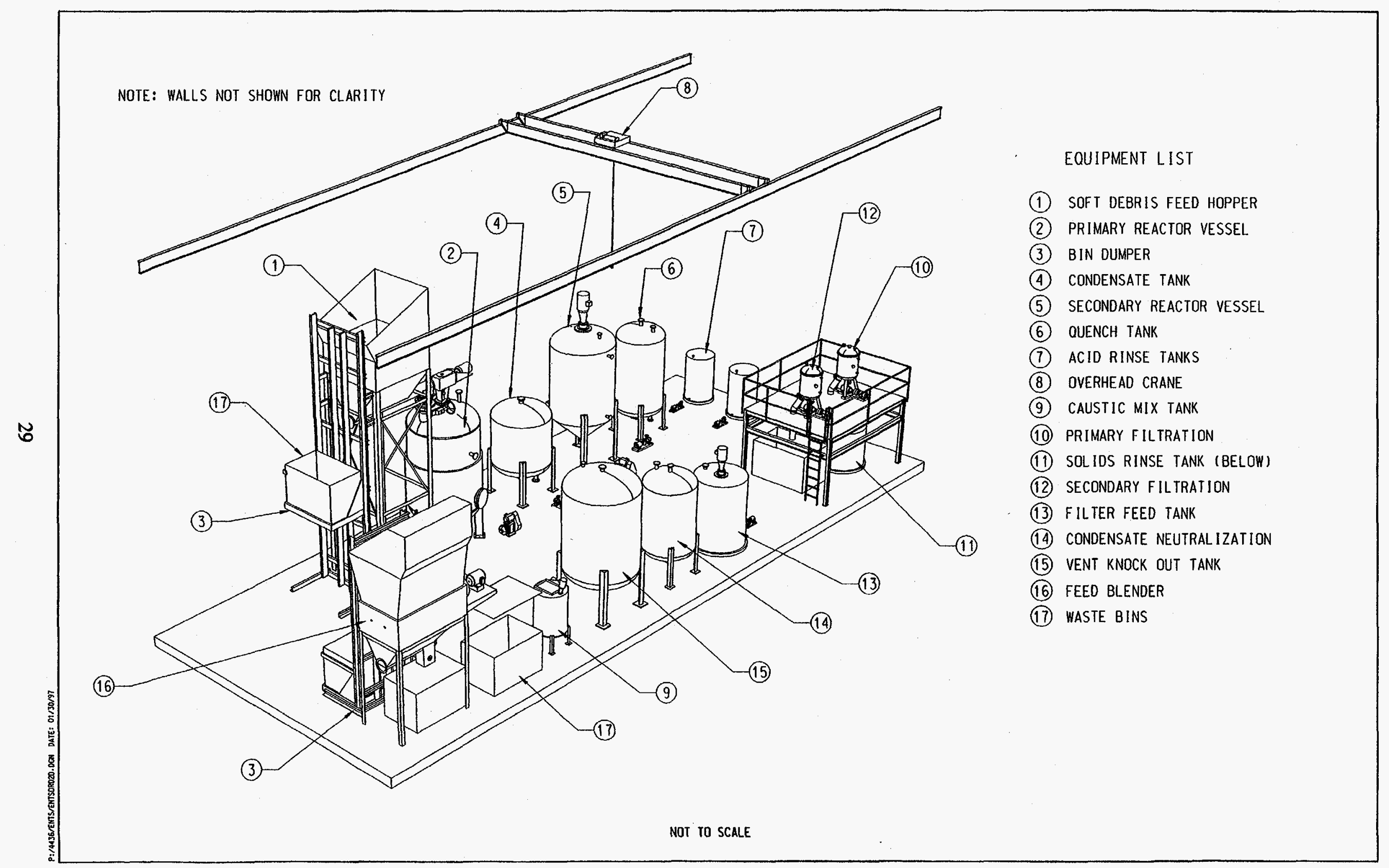

Figure 2-9. ENTS System 1: Perspective View of the CWO Organic Destruction Subsystem. 


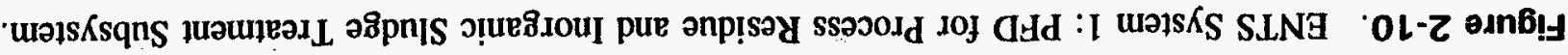

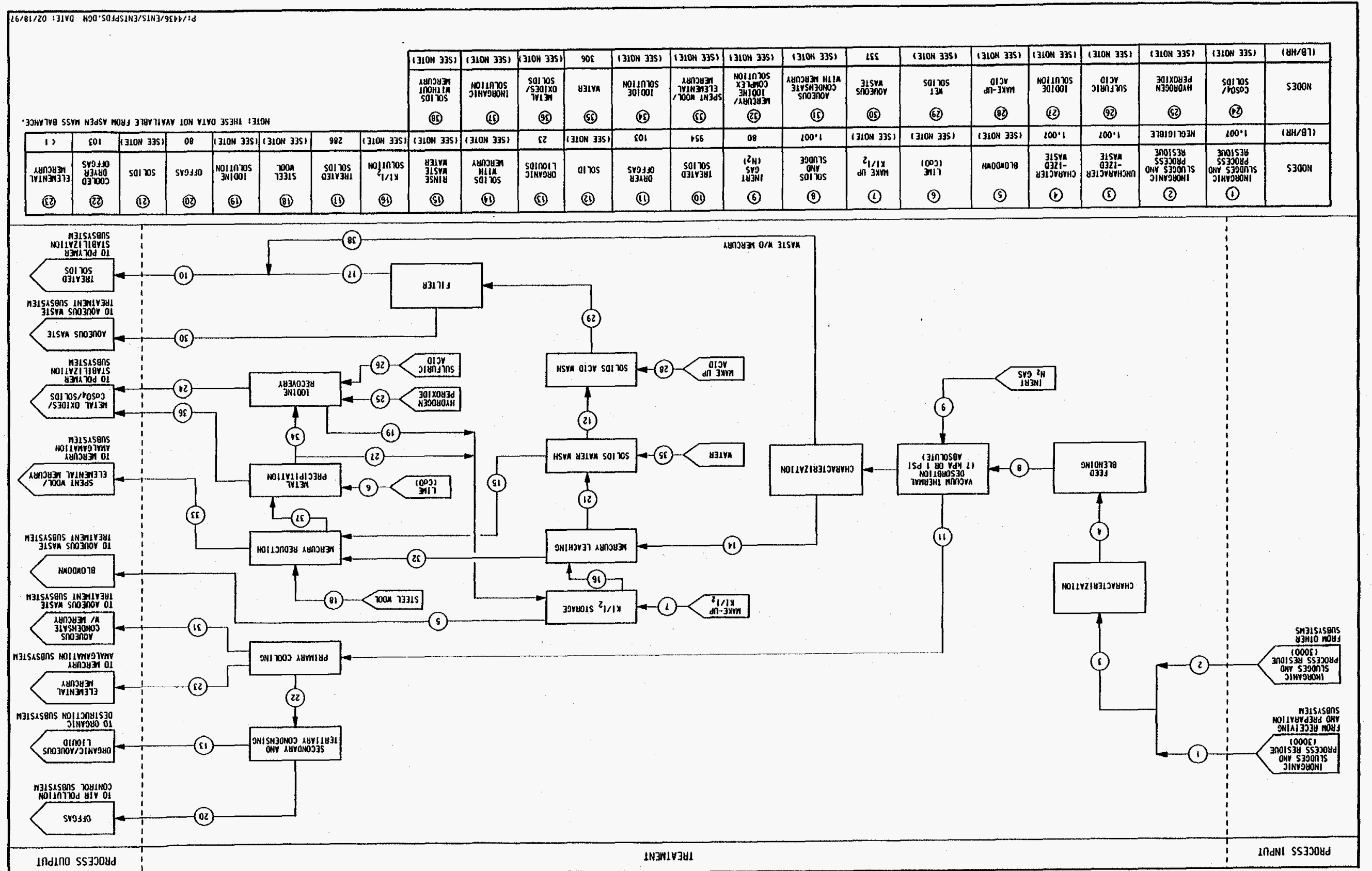




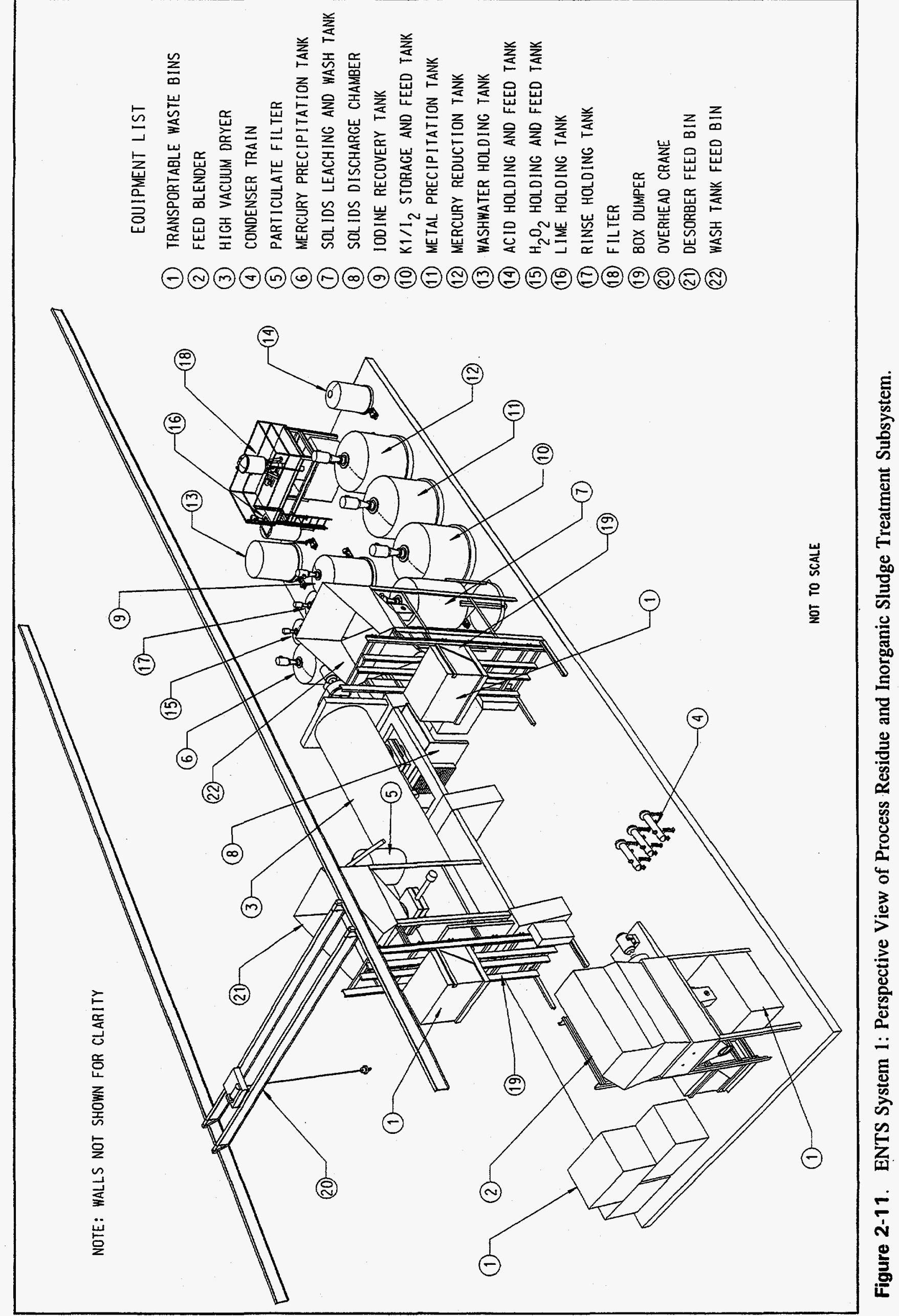




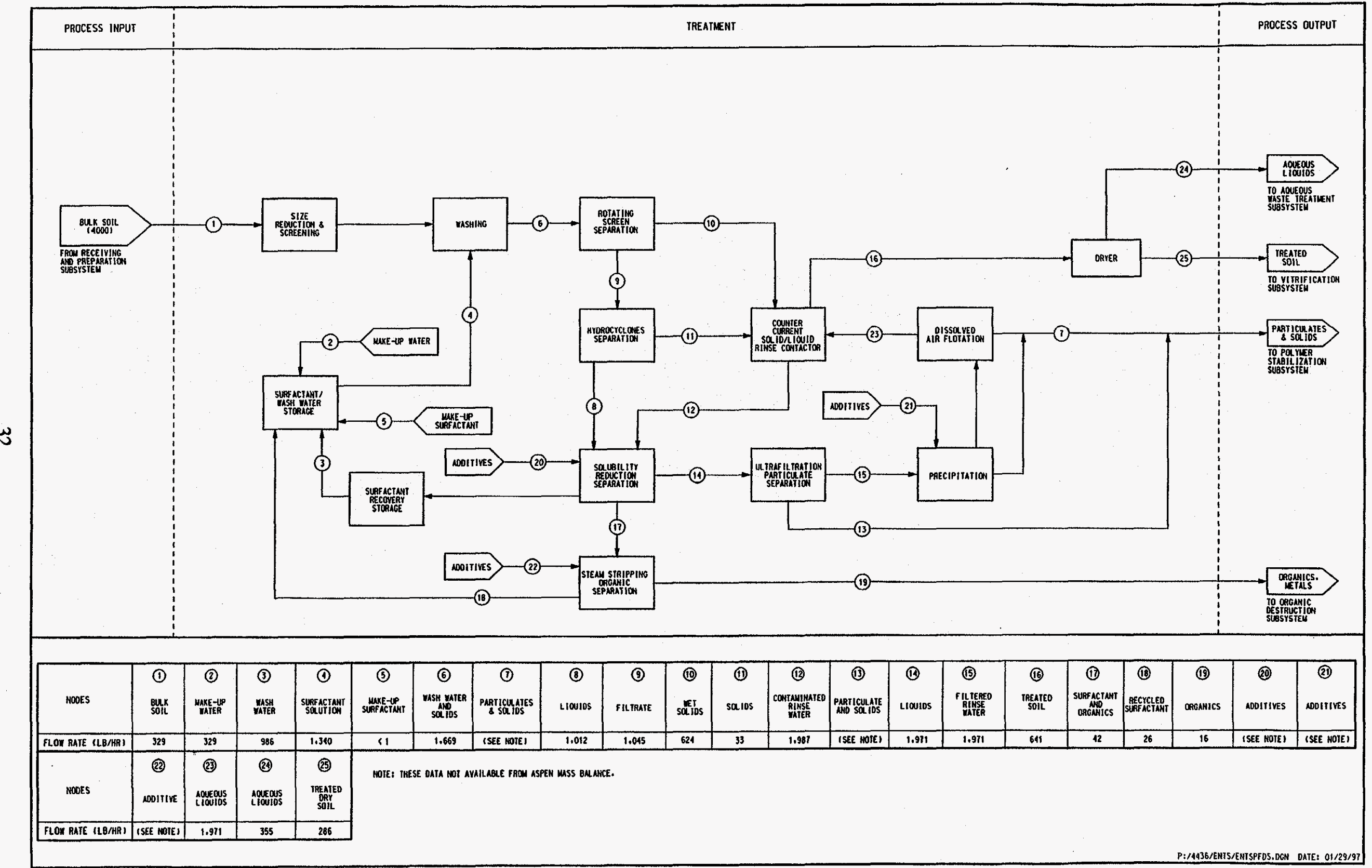

Figure 2-12. ENTS System 1: PFD for Bulk Soil Treatment Subsystem. 


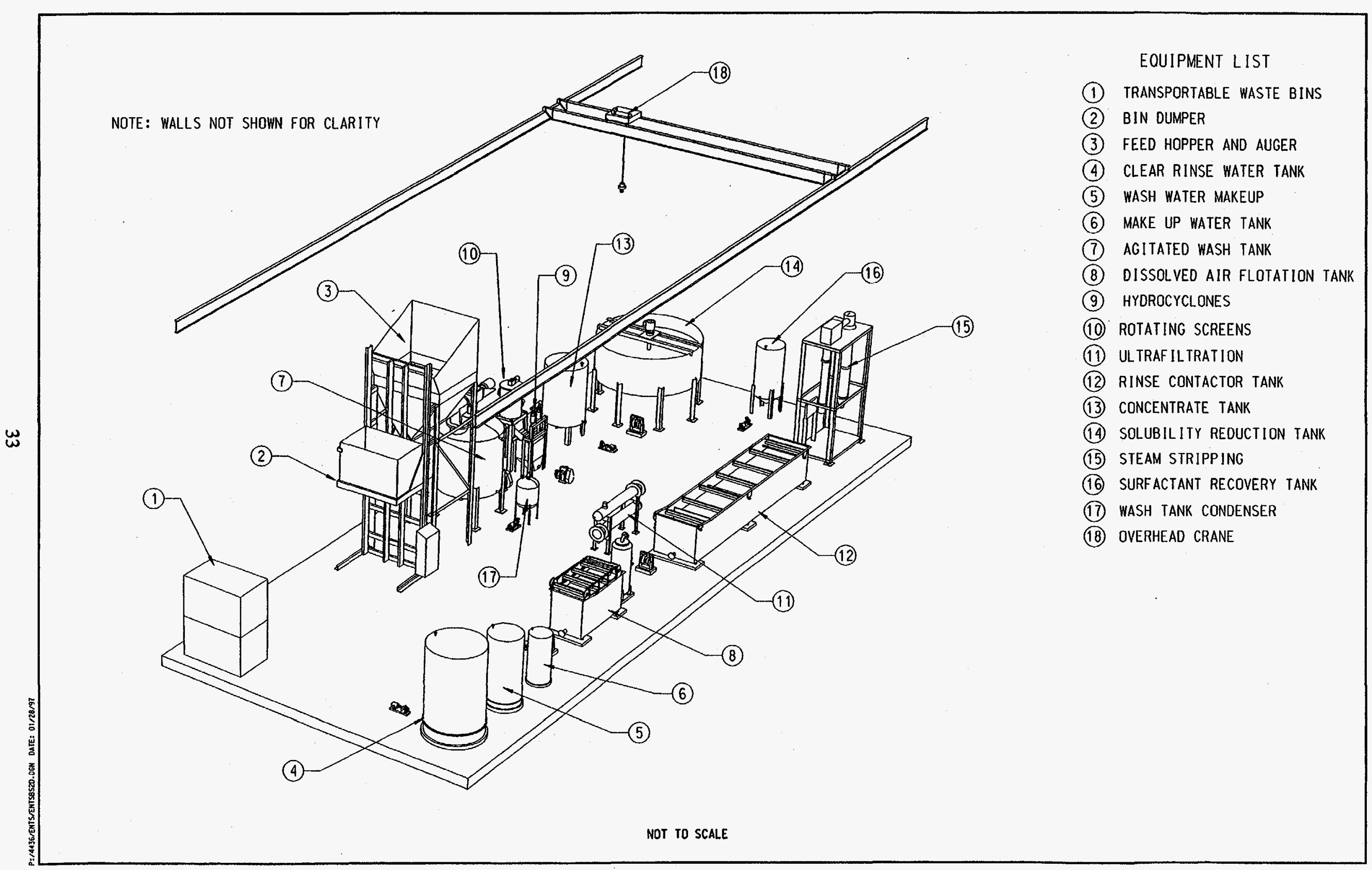

Figure 2-13. ENTS System 1: Perspective View of Bulk Soil Treatment Subsystem. 


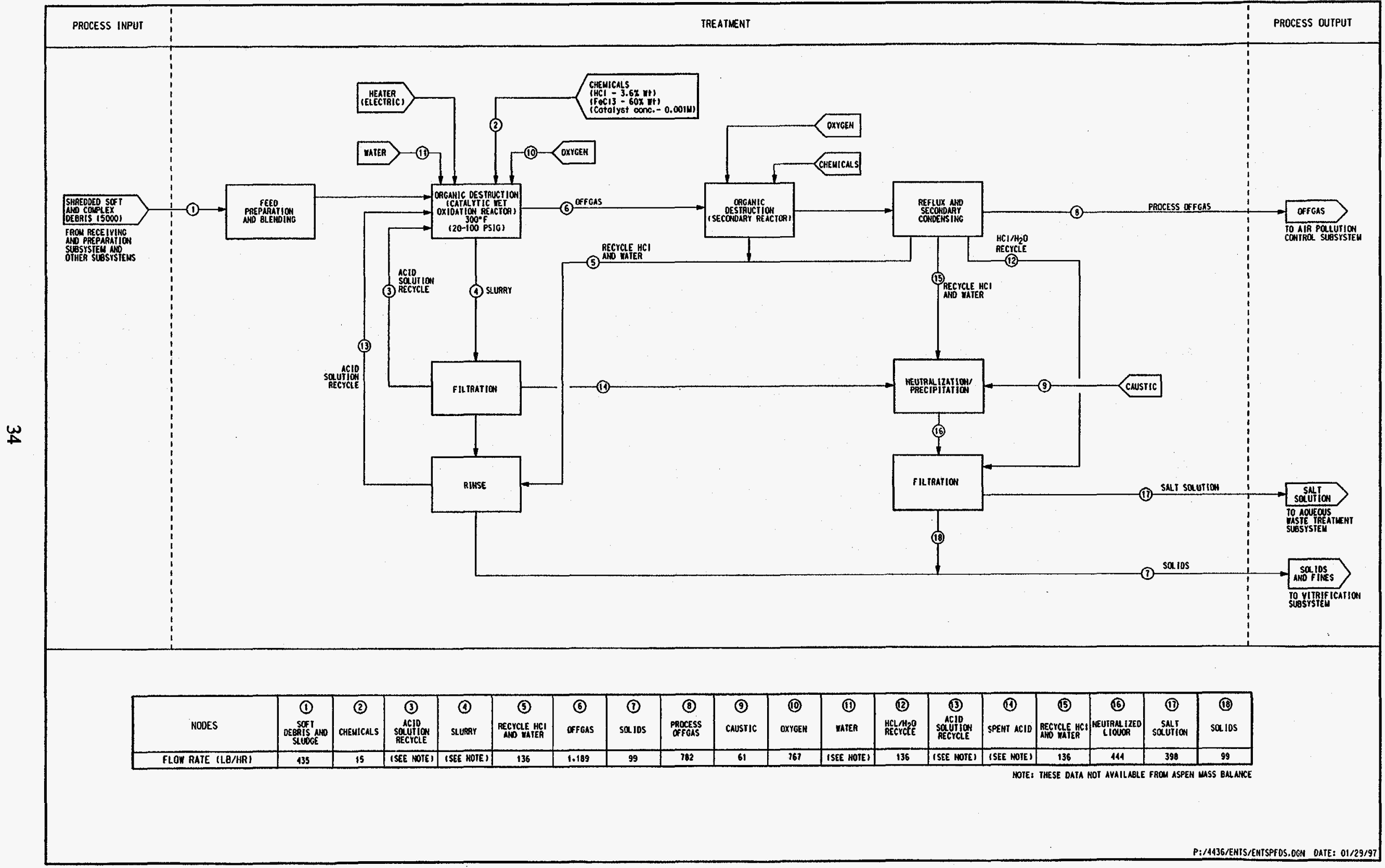

Figure 2-14. ENTS System 1: PFD for Soft Debris Treatment Subsystem. 


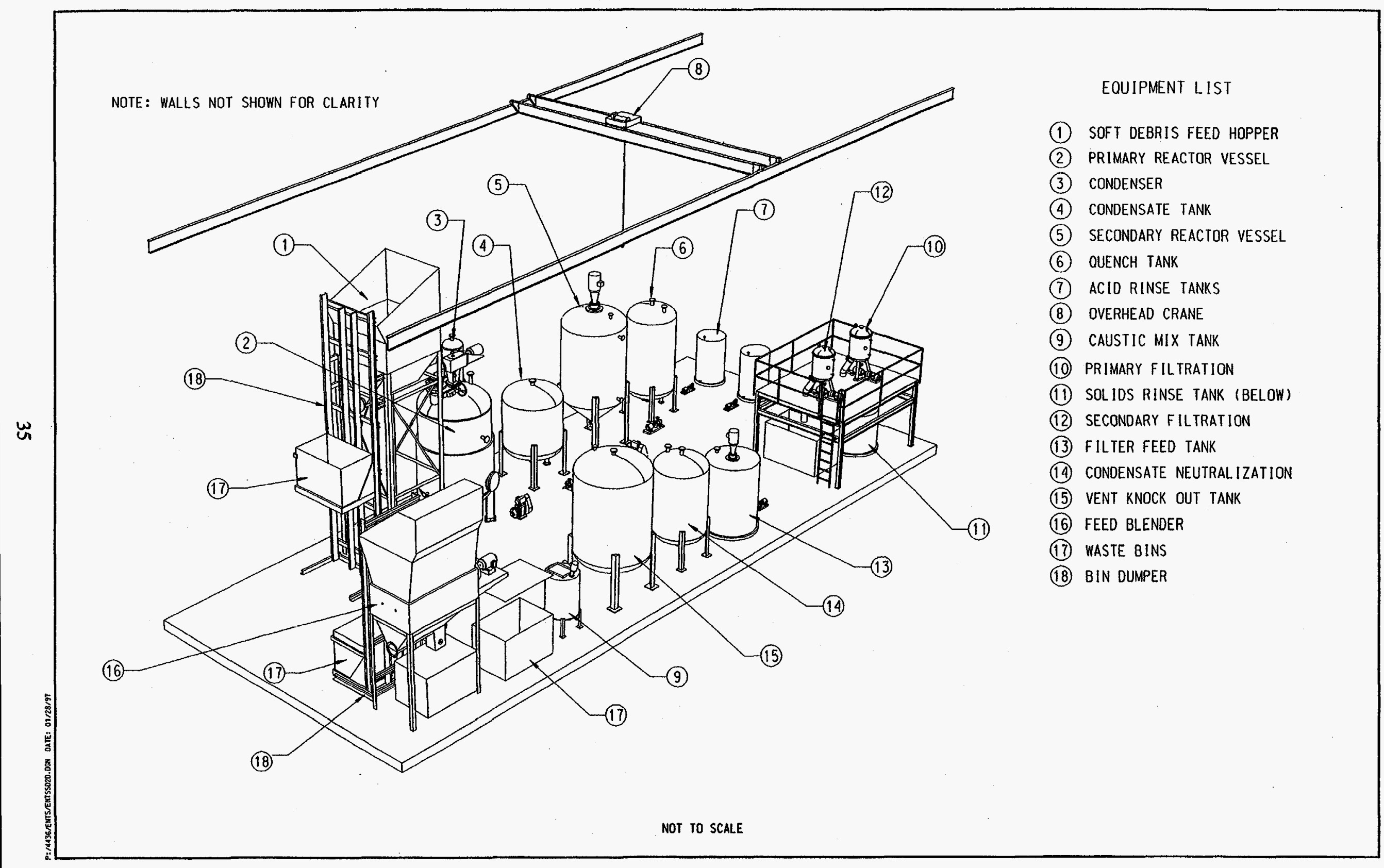

Figure 2-15. ENTS System 1: Perspective View of Soft Debris Treatment Subsystem. 


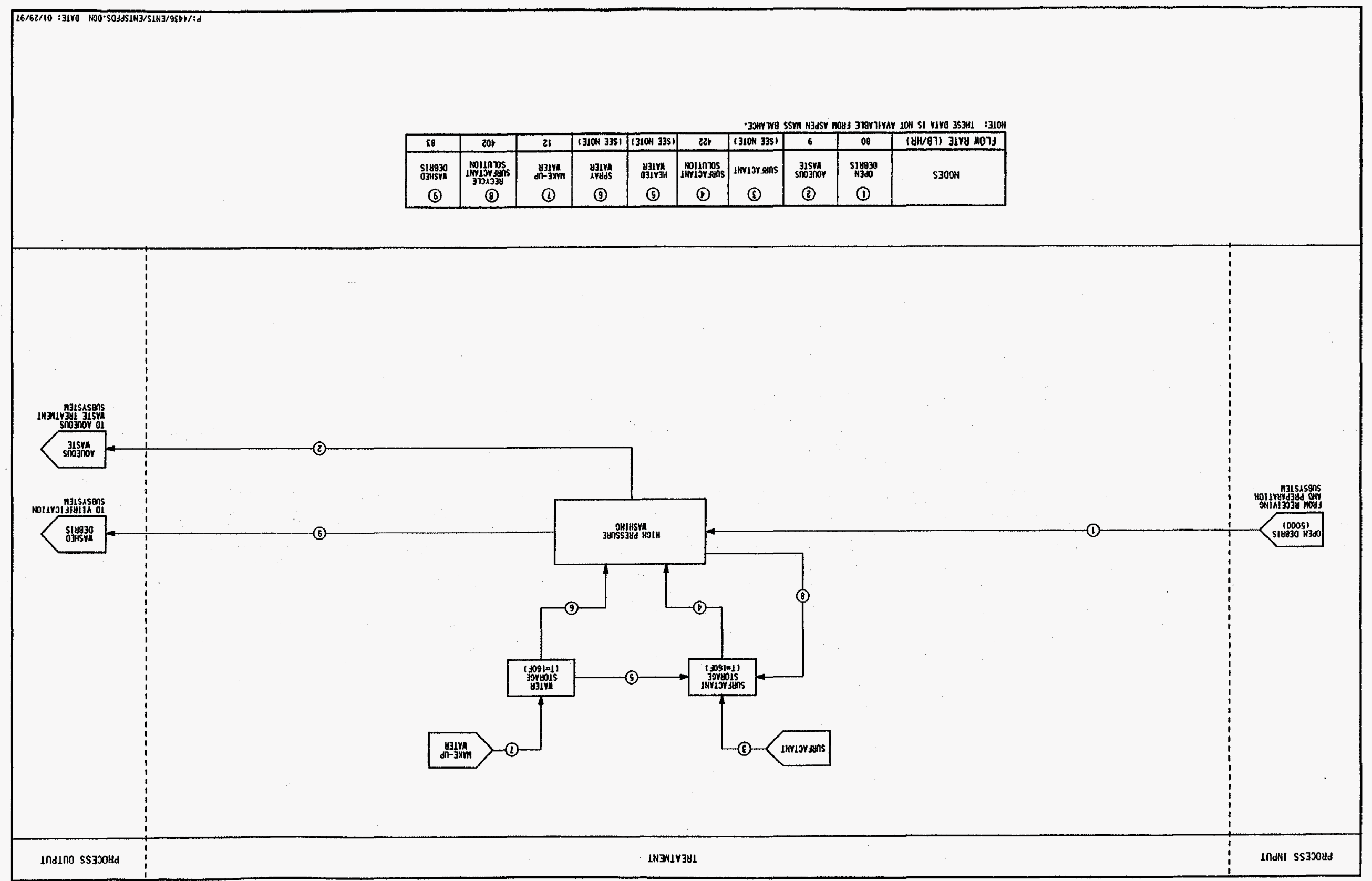



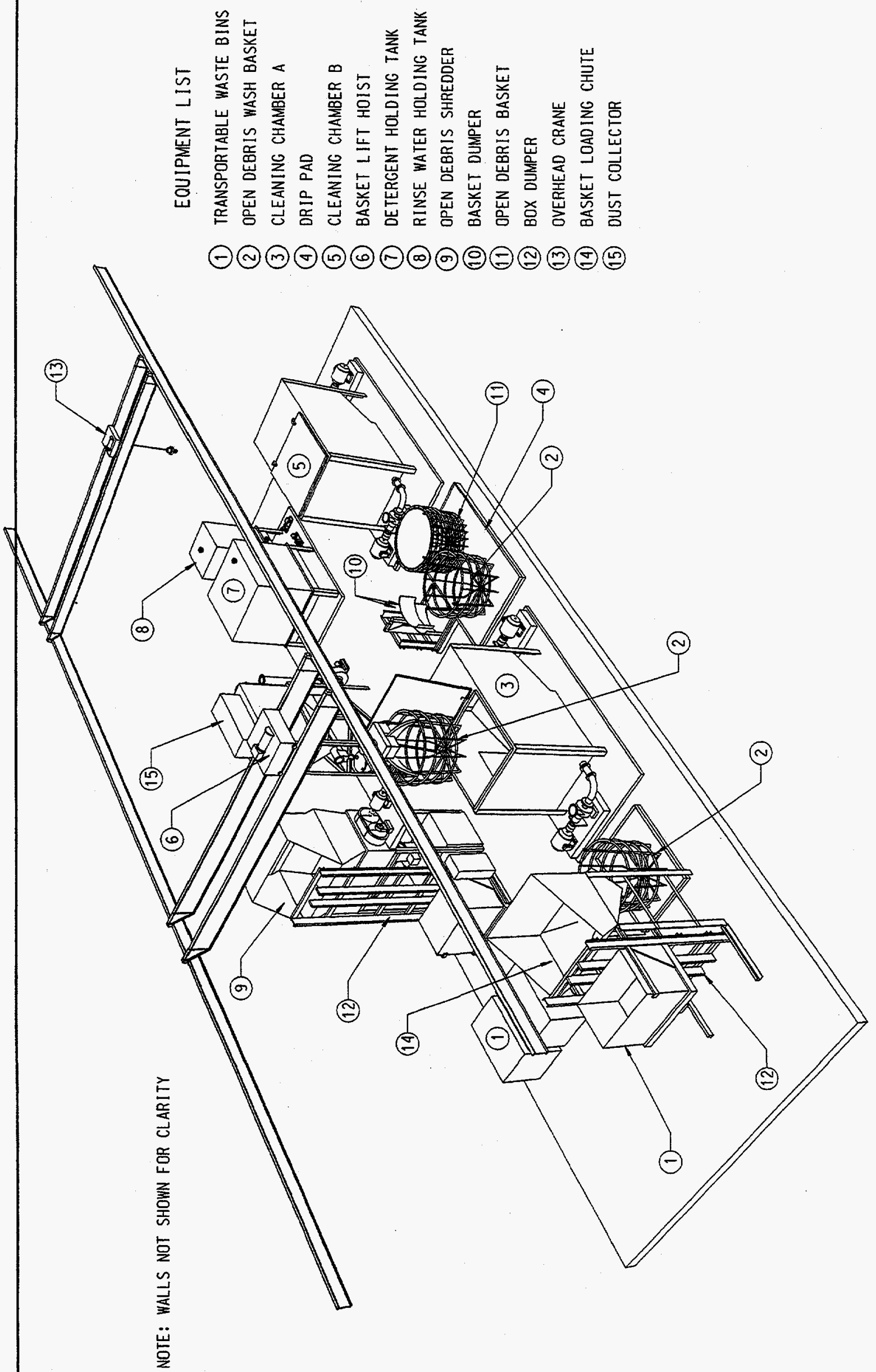


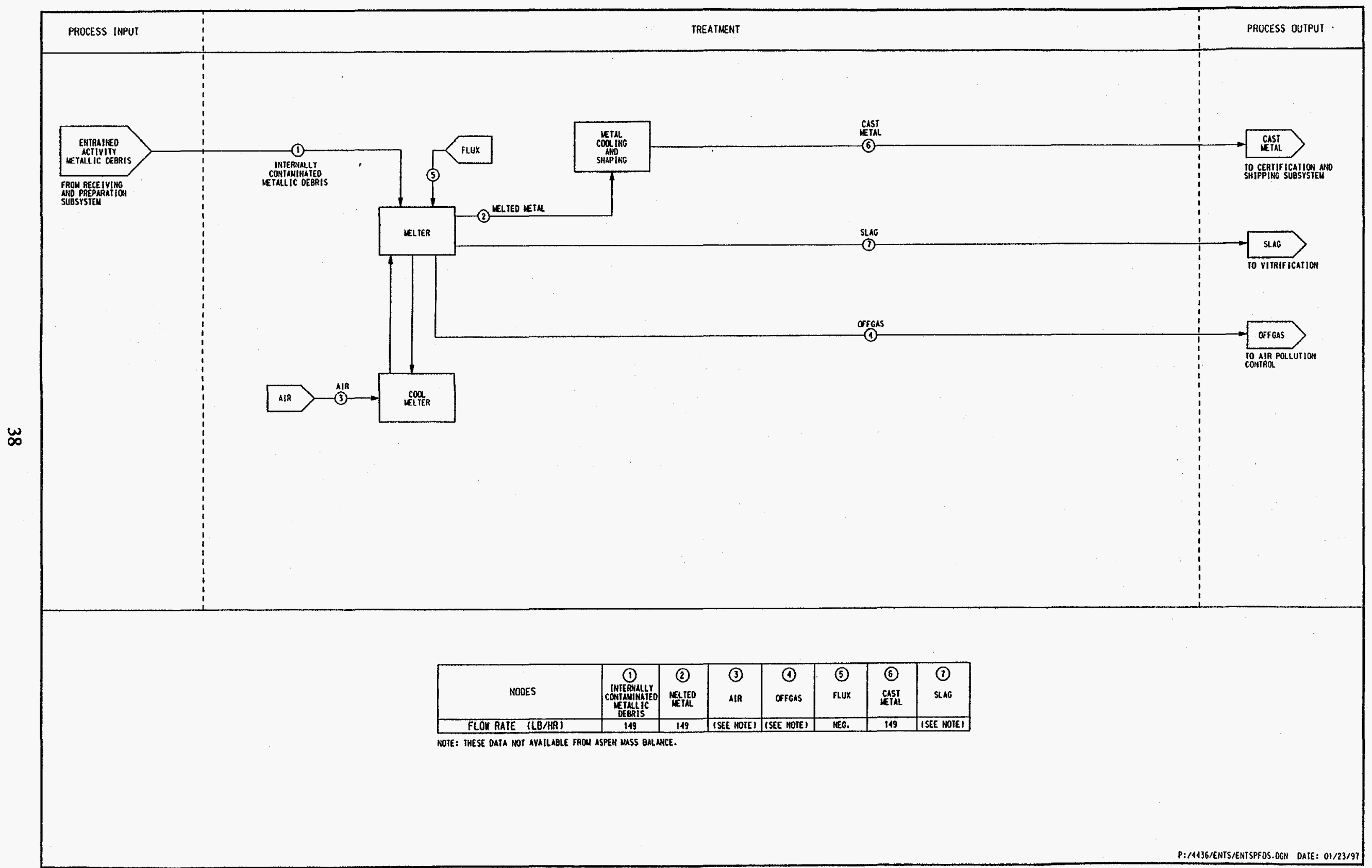

Figure 2-18. ENTS System 1: PFD for Metal Meiting Subsystem. 


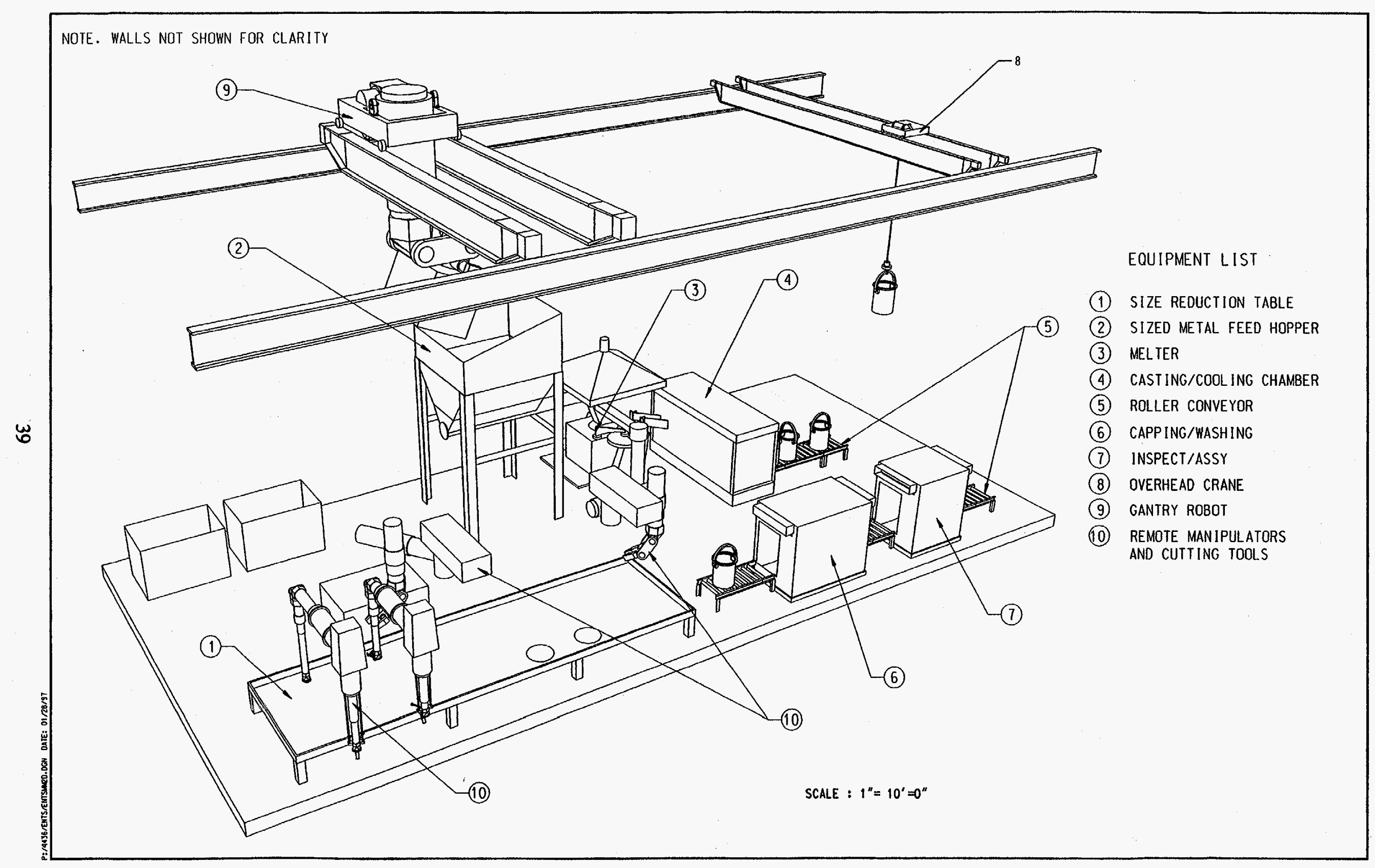

Figure 2-19. ENTS System 1: Perspective View of Metal Melting Subsystem. 


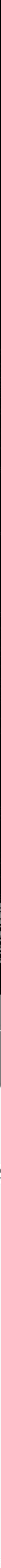

Figure 2-20. ENTS System 1: PFD for Metal Decontamination Subsystem. 


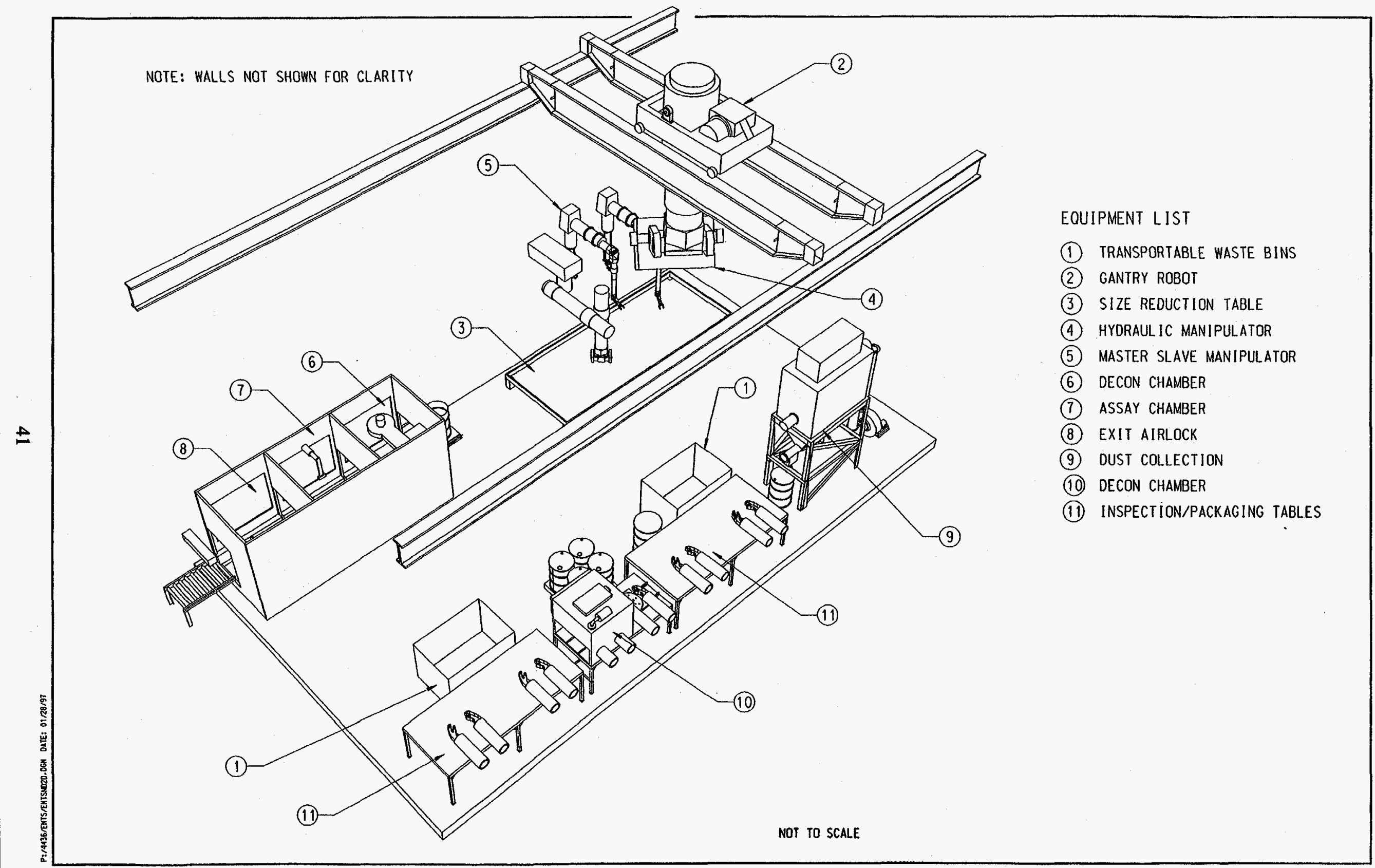

Figure 2-21. ENTS System 1: Perspective View of Metal Decontamination Subsystem. 


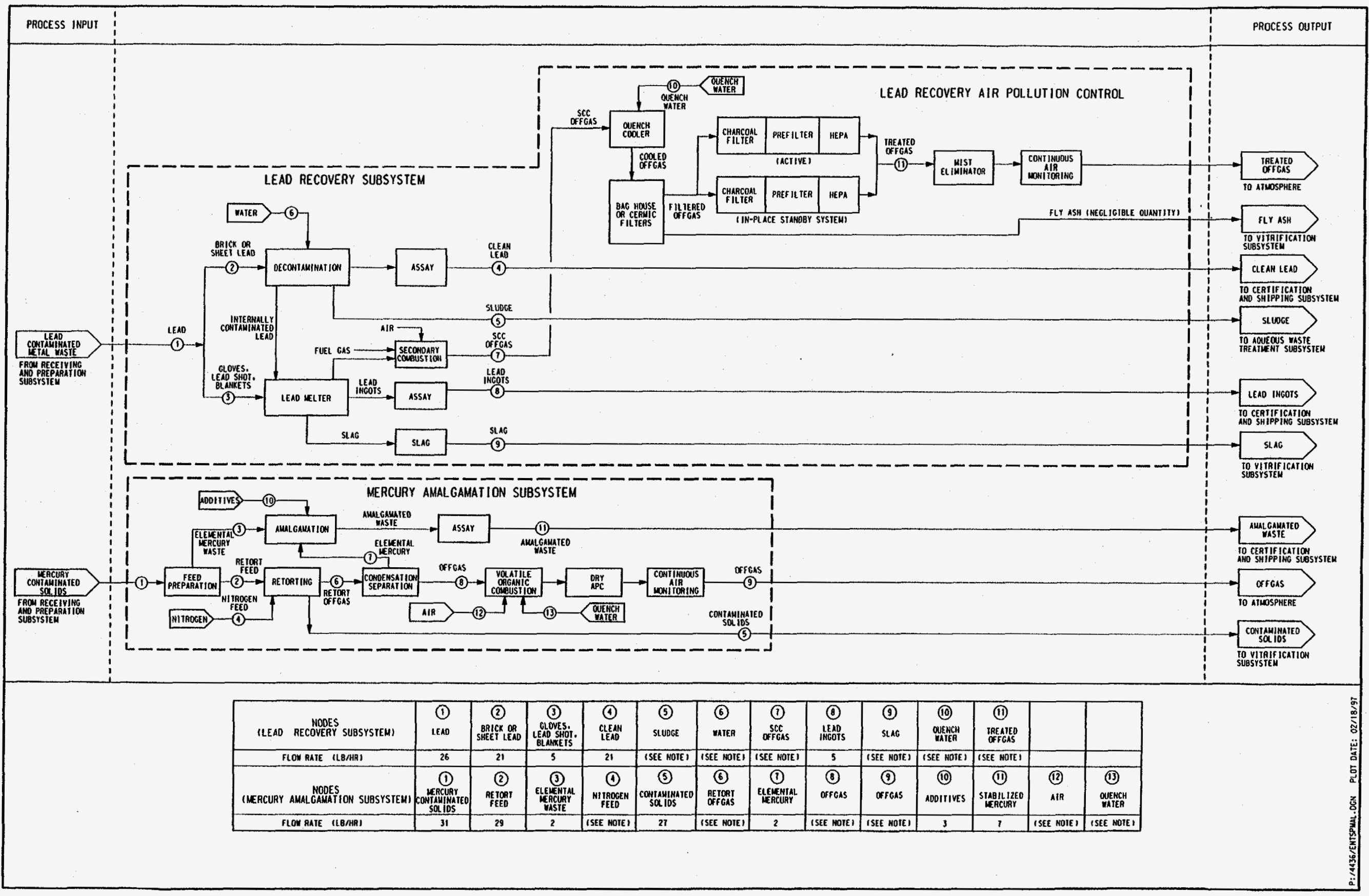

Figure 2-22. ENTS System 1: PFD for Mercury Amalgamation and Lead Recovery Subsystems. 


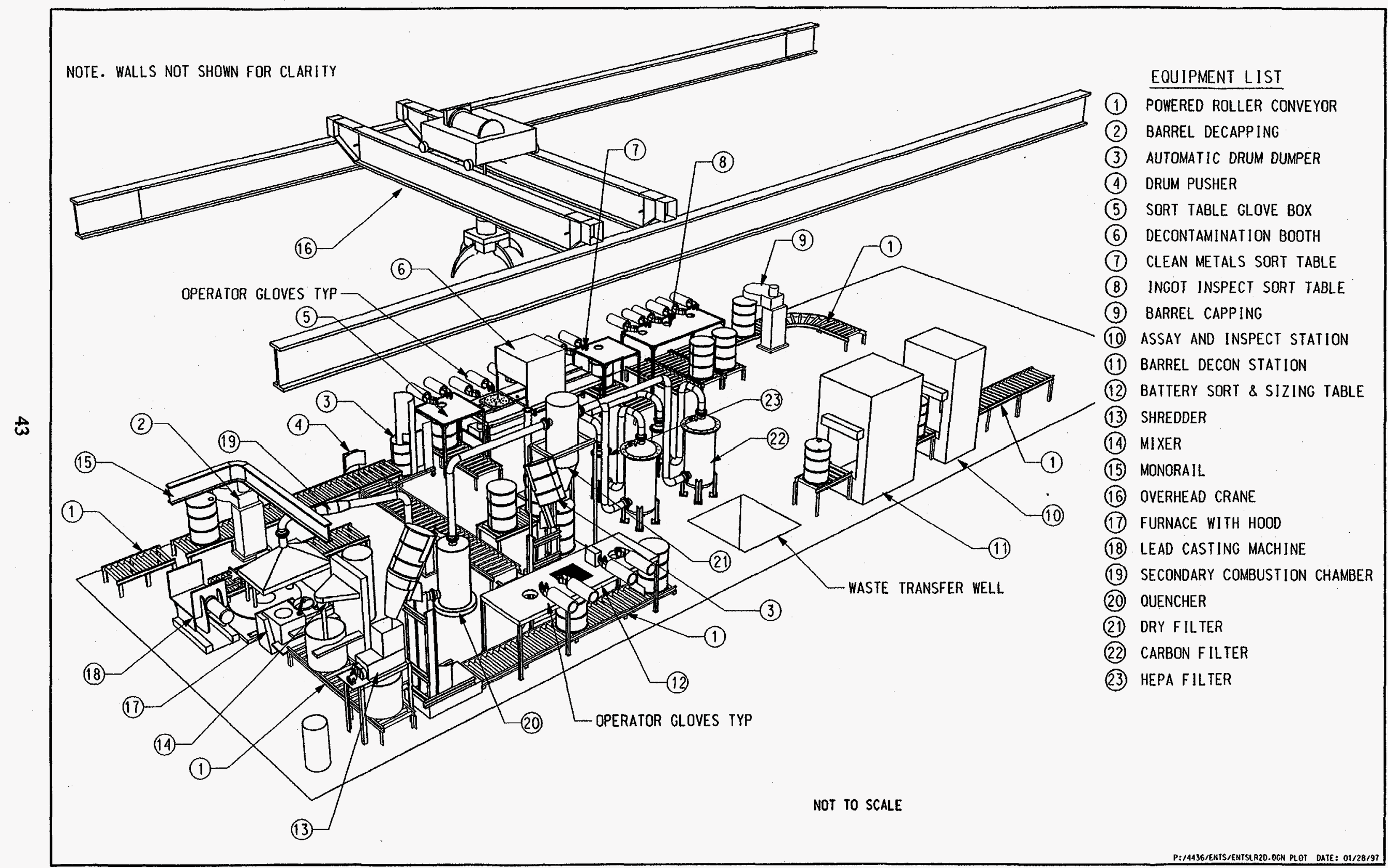

Figure 2-23. ENTS System 1: Perspective View of Lead Recovery Subsystem. 


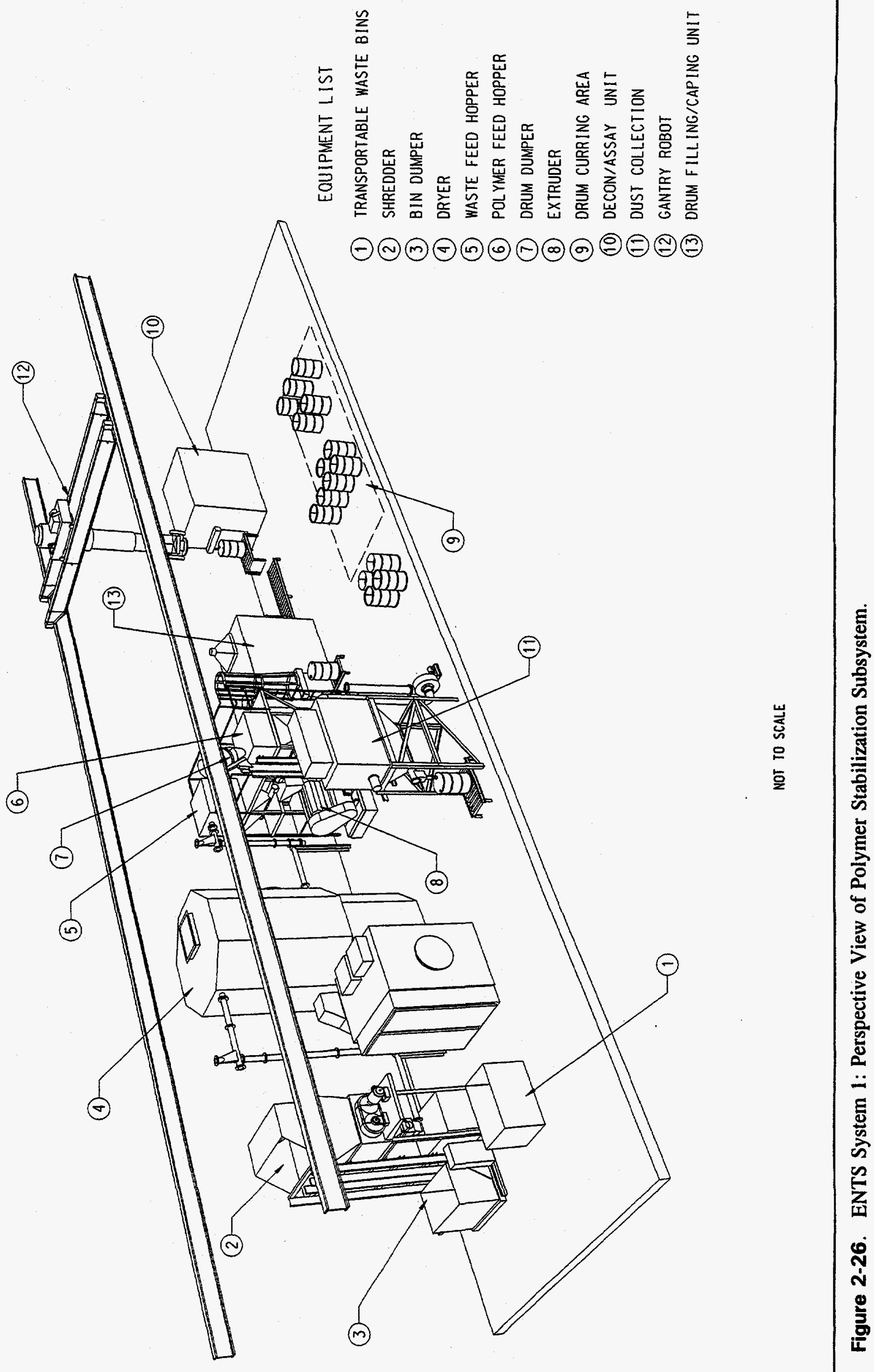




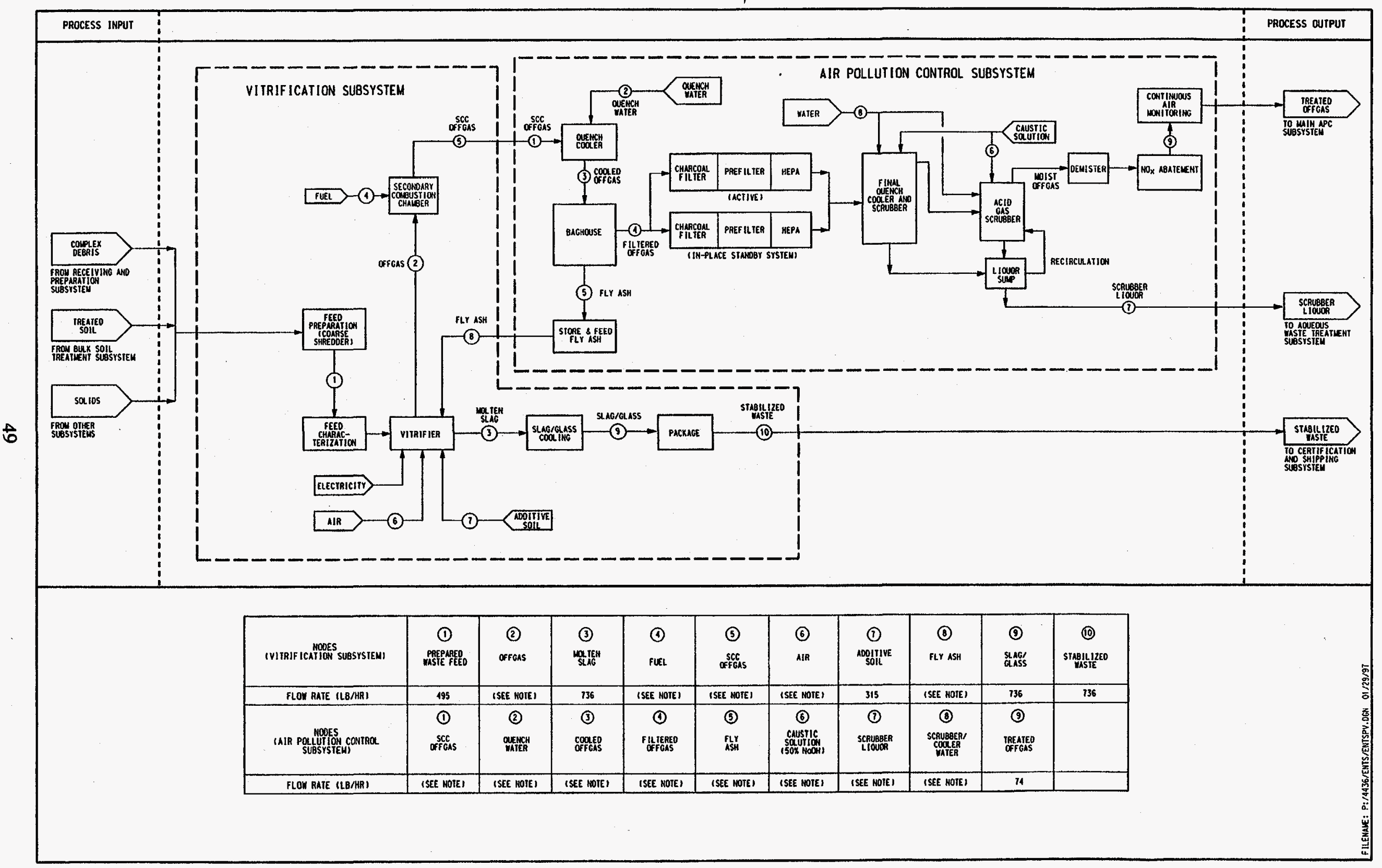

Figure 2-29. ENTS System 1: PFD for Vitrification Subsystem. 


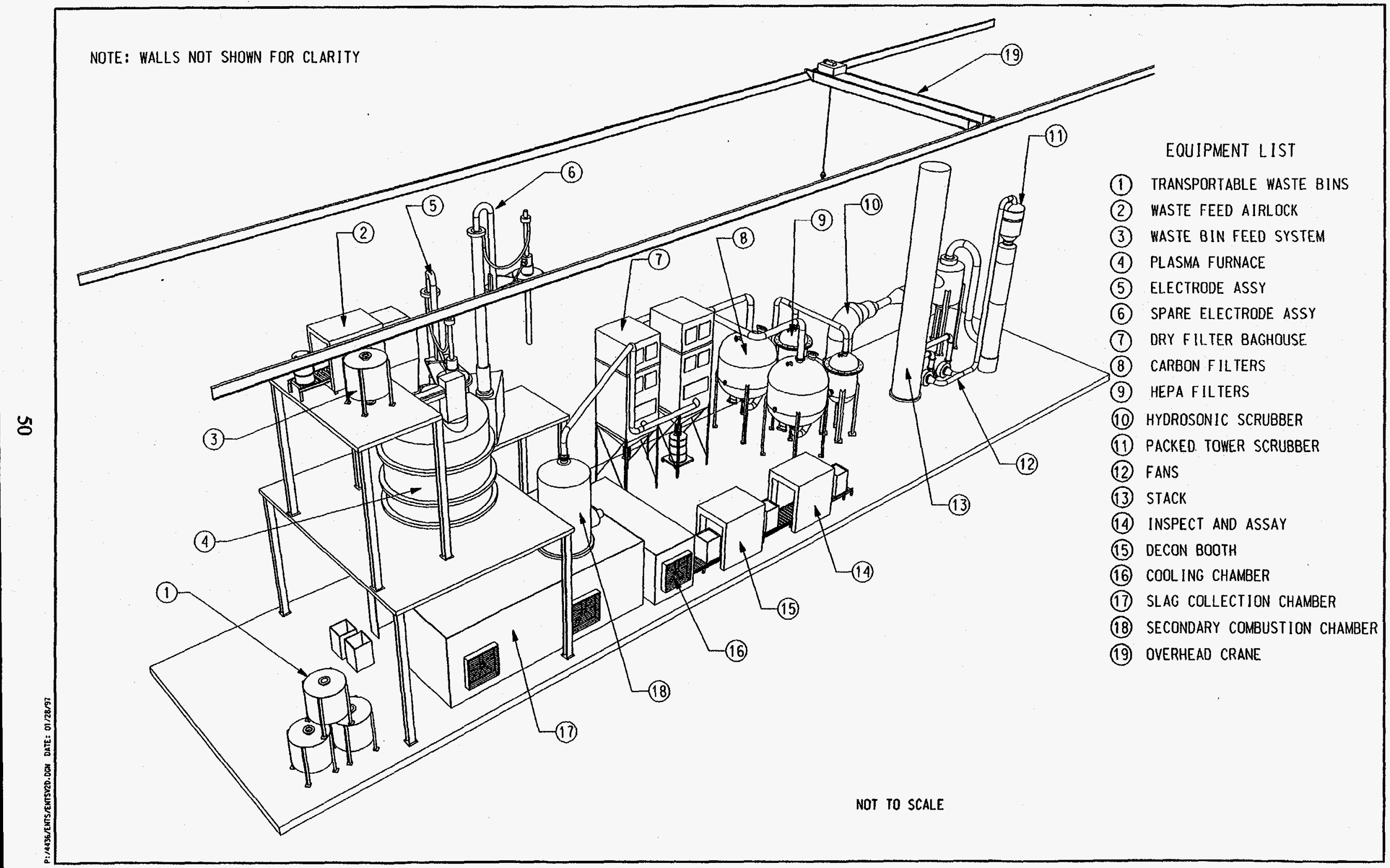

Figure 2-30. ENTS System 1: Perspective View of Vitrification Subsystem. 


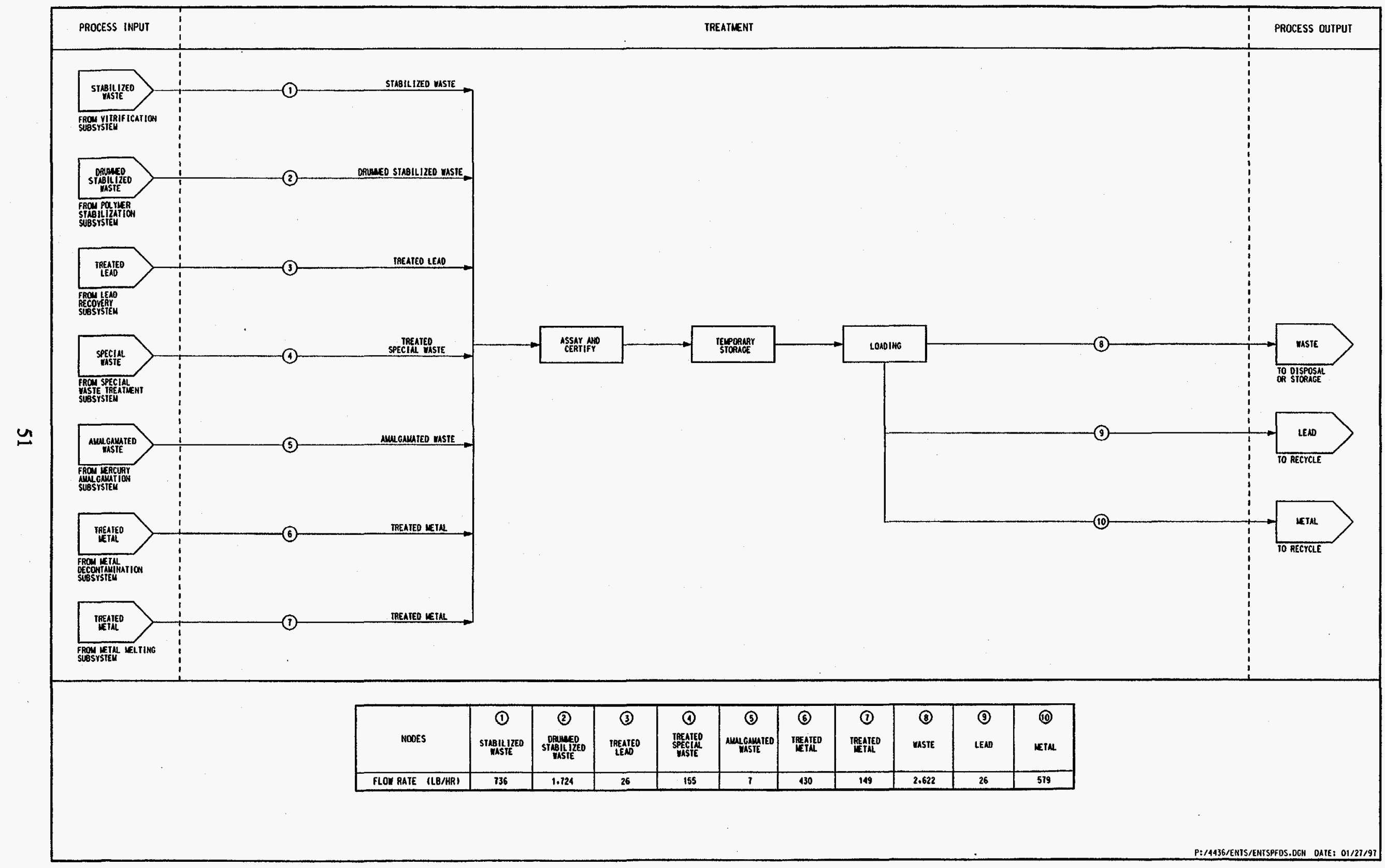

Figure 2-31. ENTS System 1: PFD for Certification and Shipping Subsystem. 


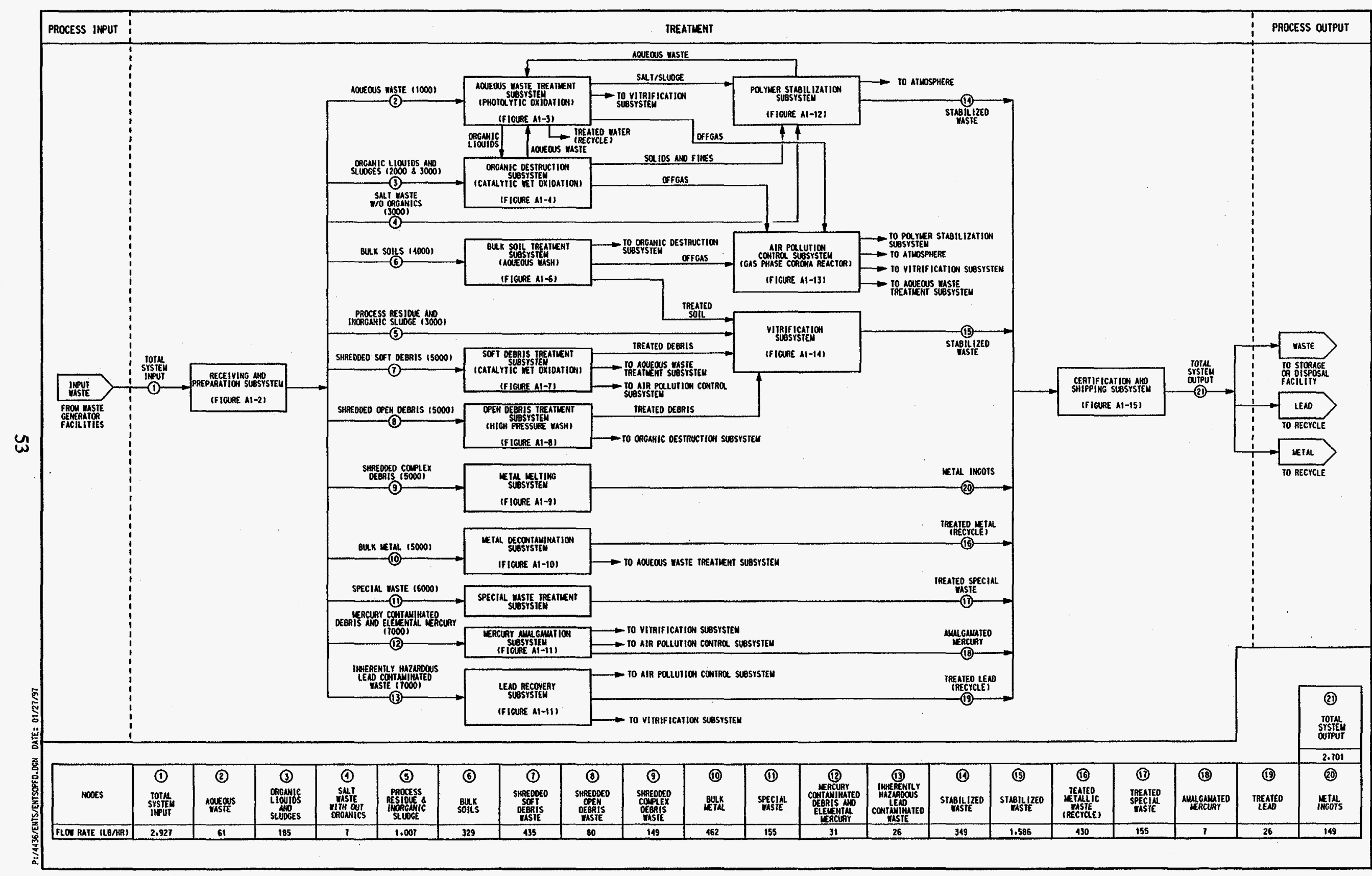

Figure 2-33. ENTS System 2: PFD for Overall System. 


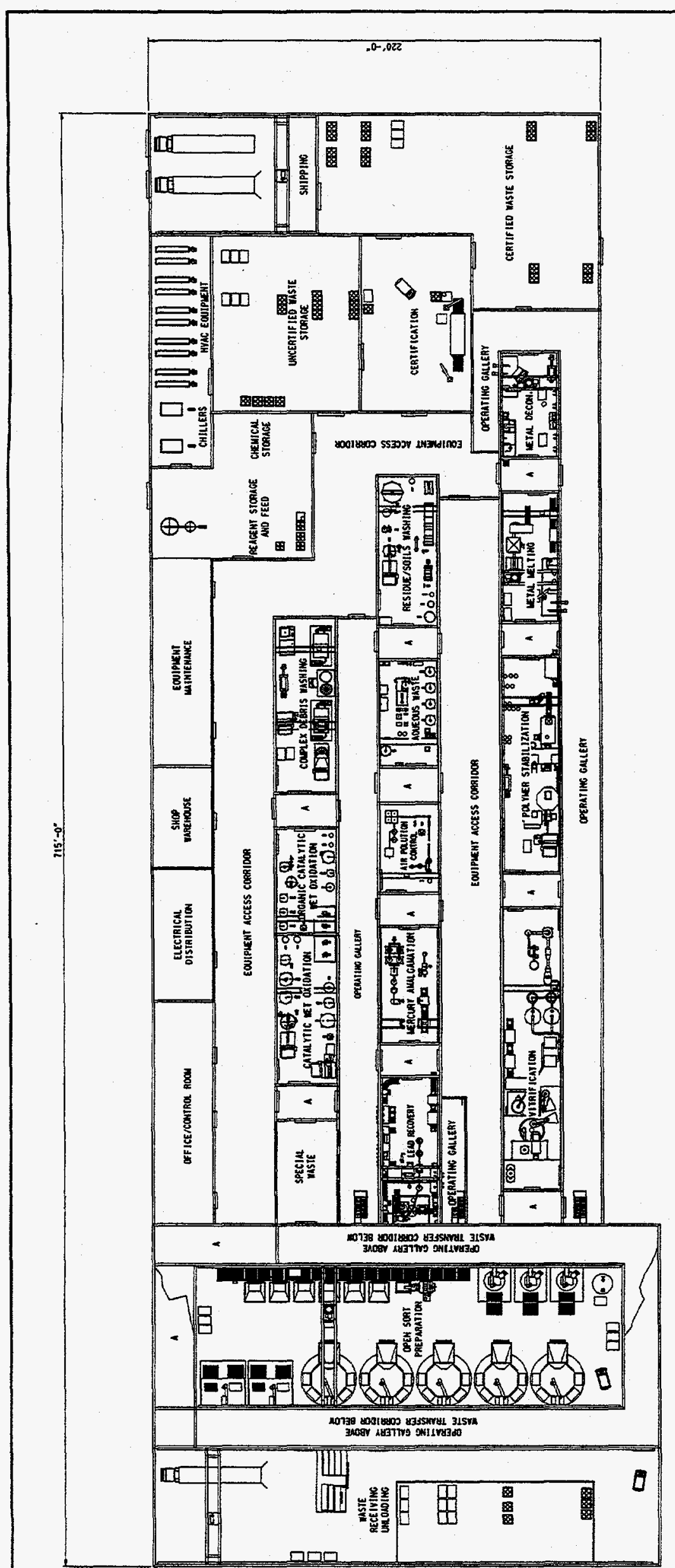

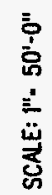

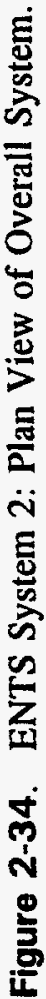




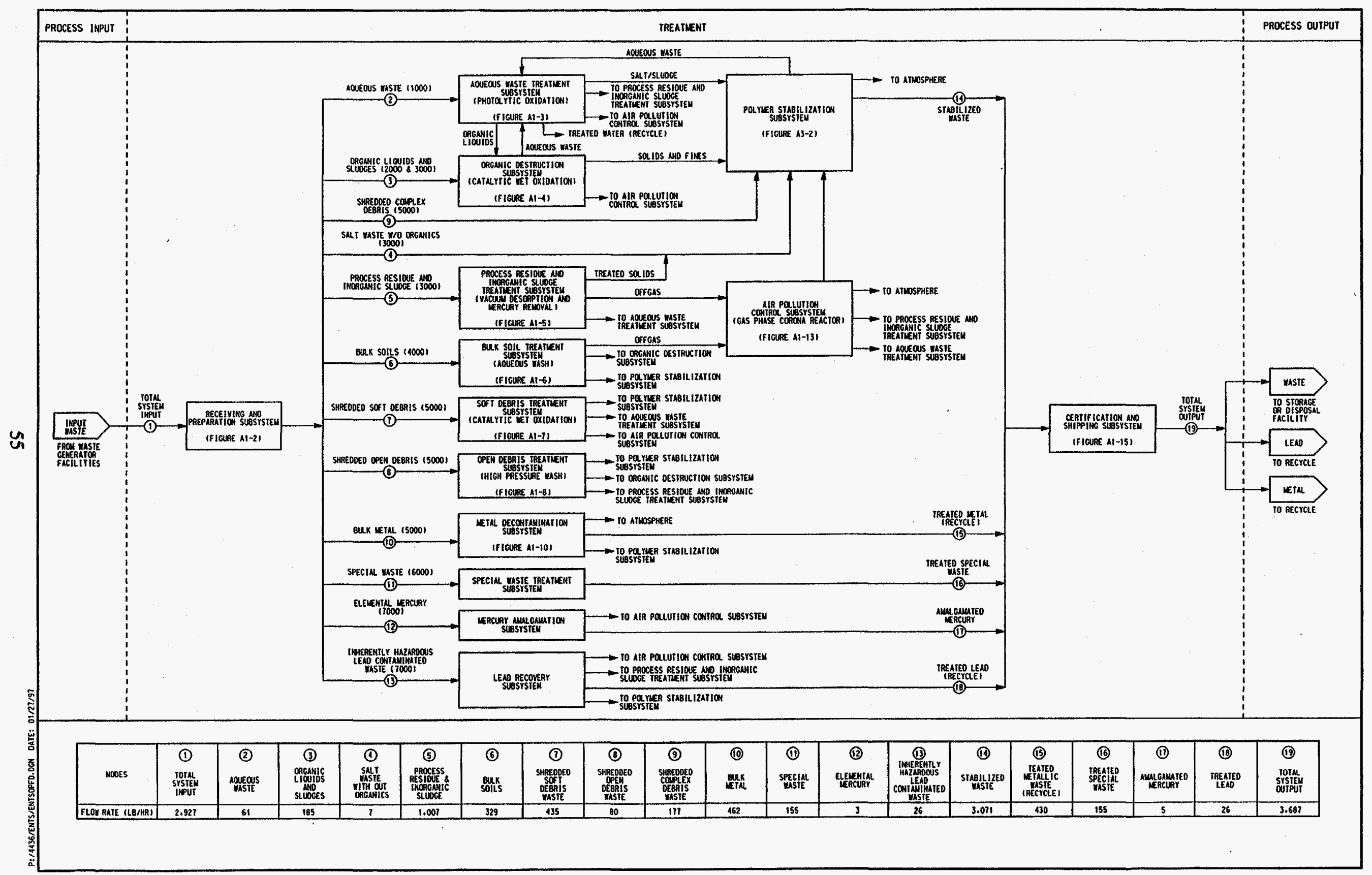

Figure 2-35. ENTS System 3: PFD for Overall System. 


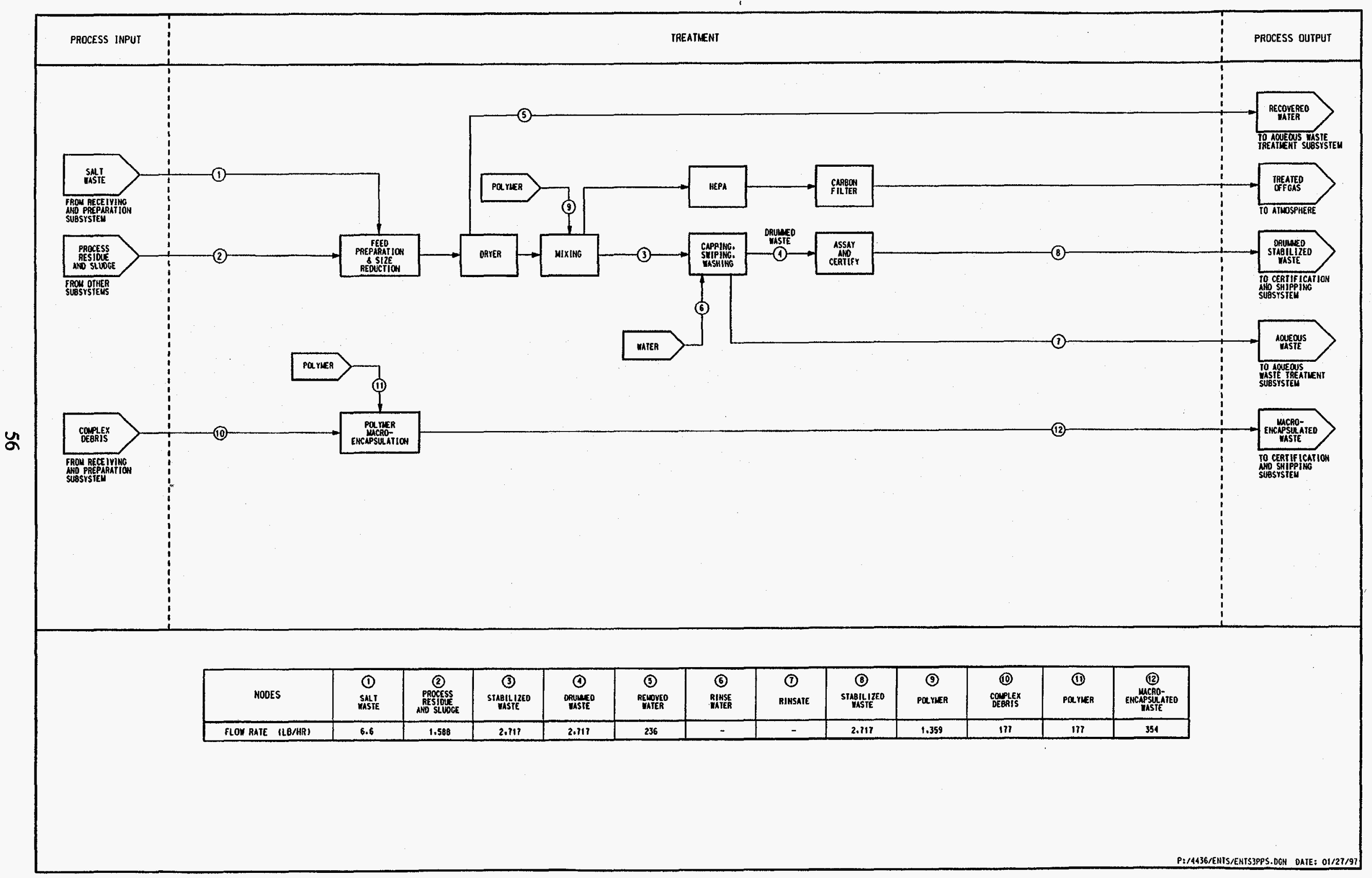

Figure 2-36. ENTS System 3: PFD for Polymer Stabilization Subsystem. 


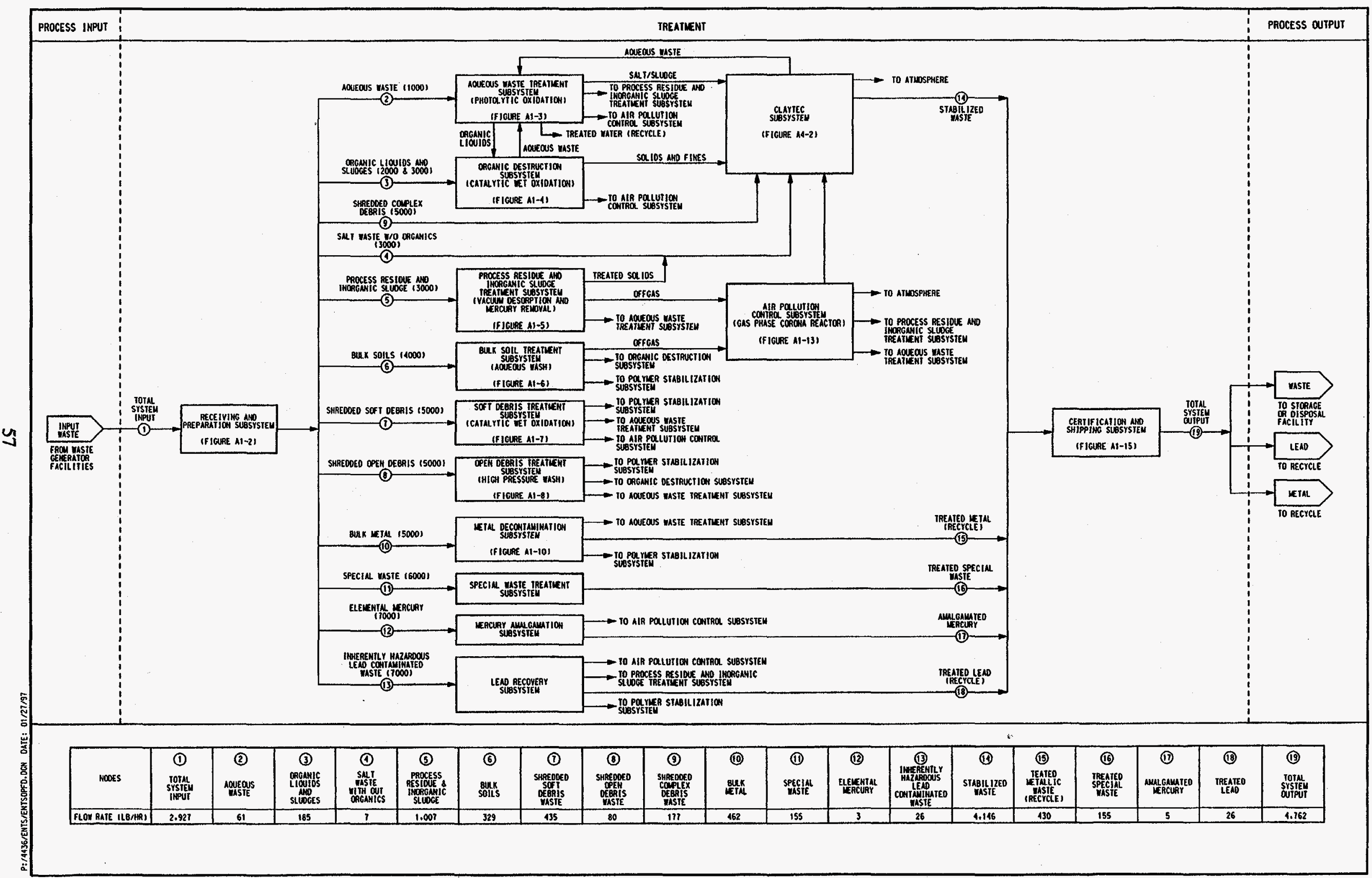

Figure 2-37. ENTS System 4: PFD for Overall System. 


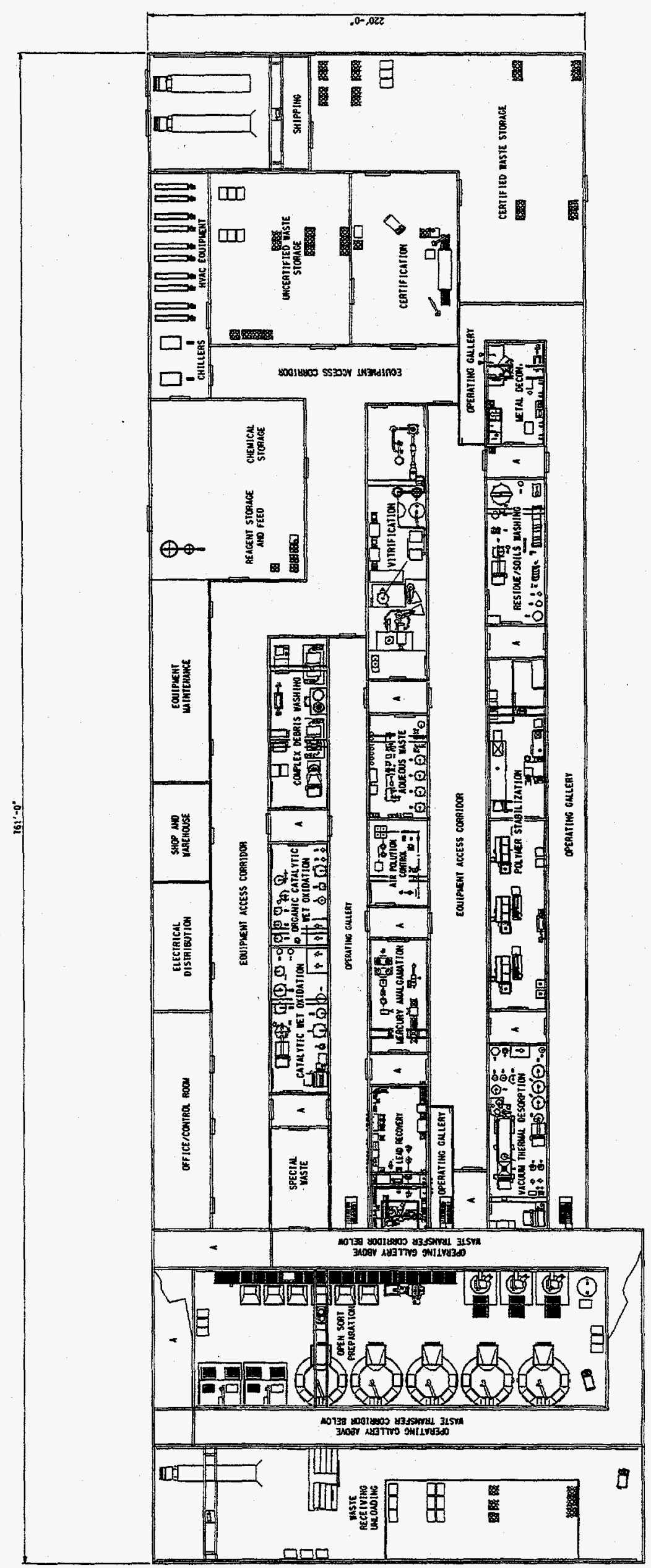

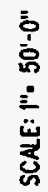




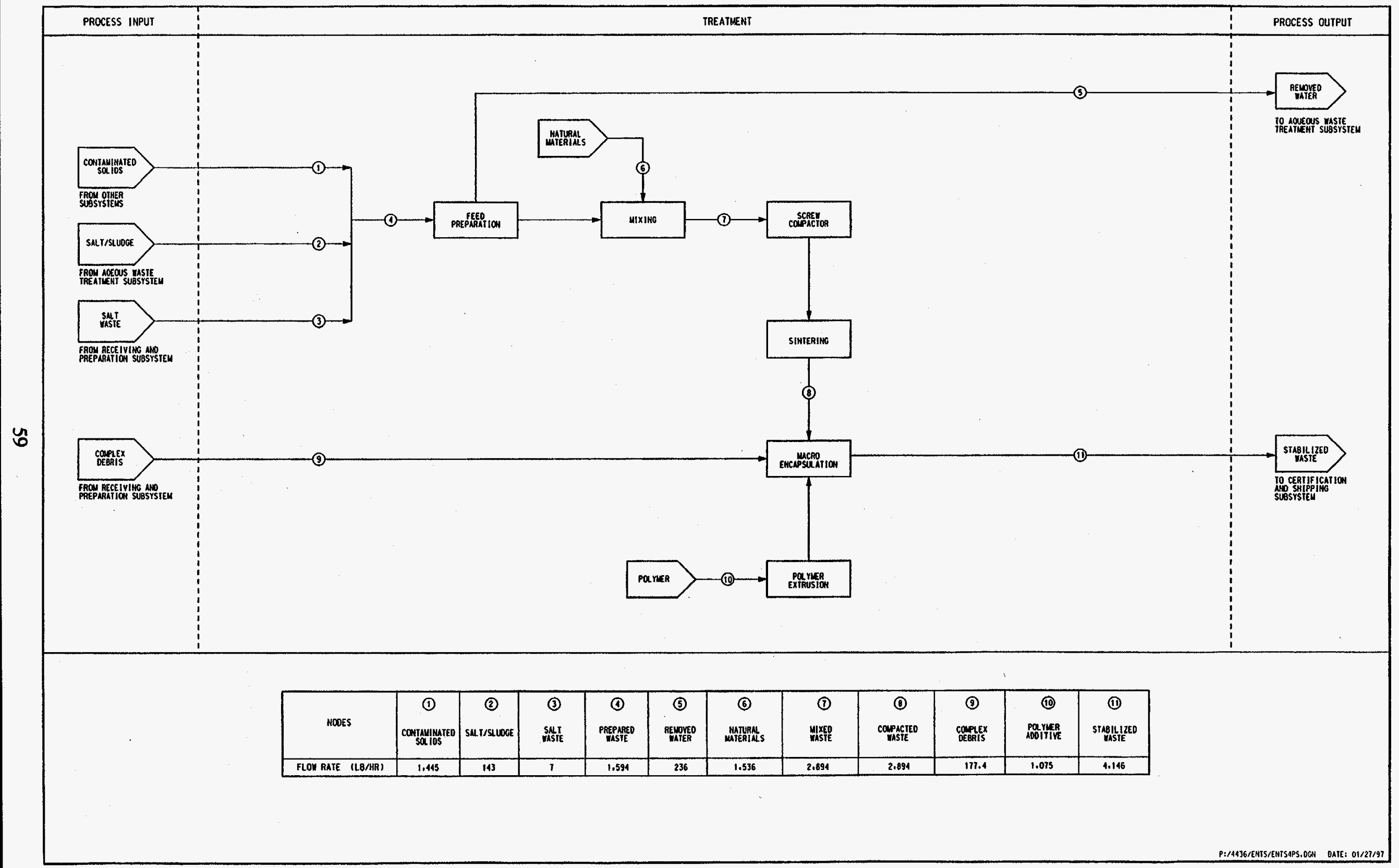

Figure 2-39. ENTS System 4: PFD for Claytec Subsystem. 


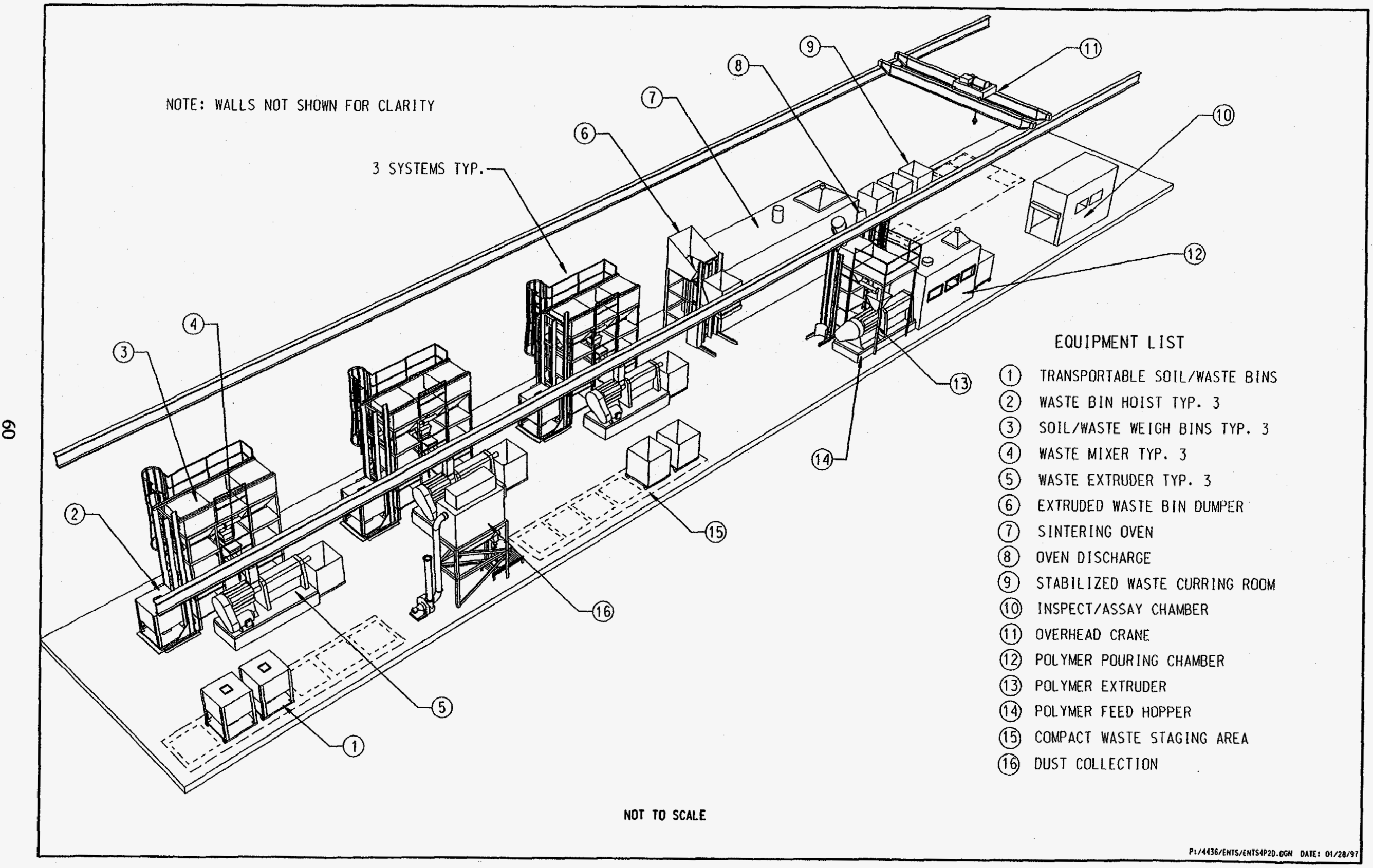

Figure 2-40. ENTS System 4: Perspective View of Claytec Subsystem. 


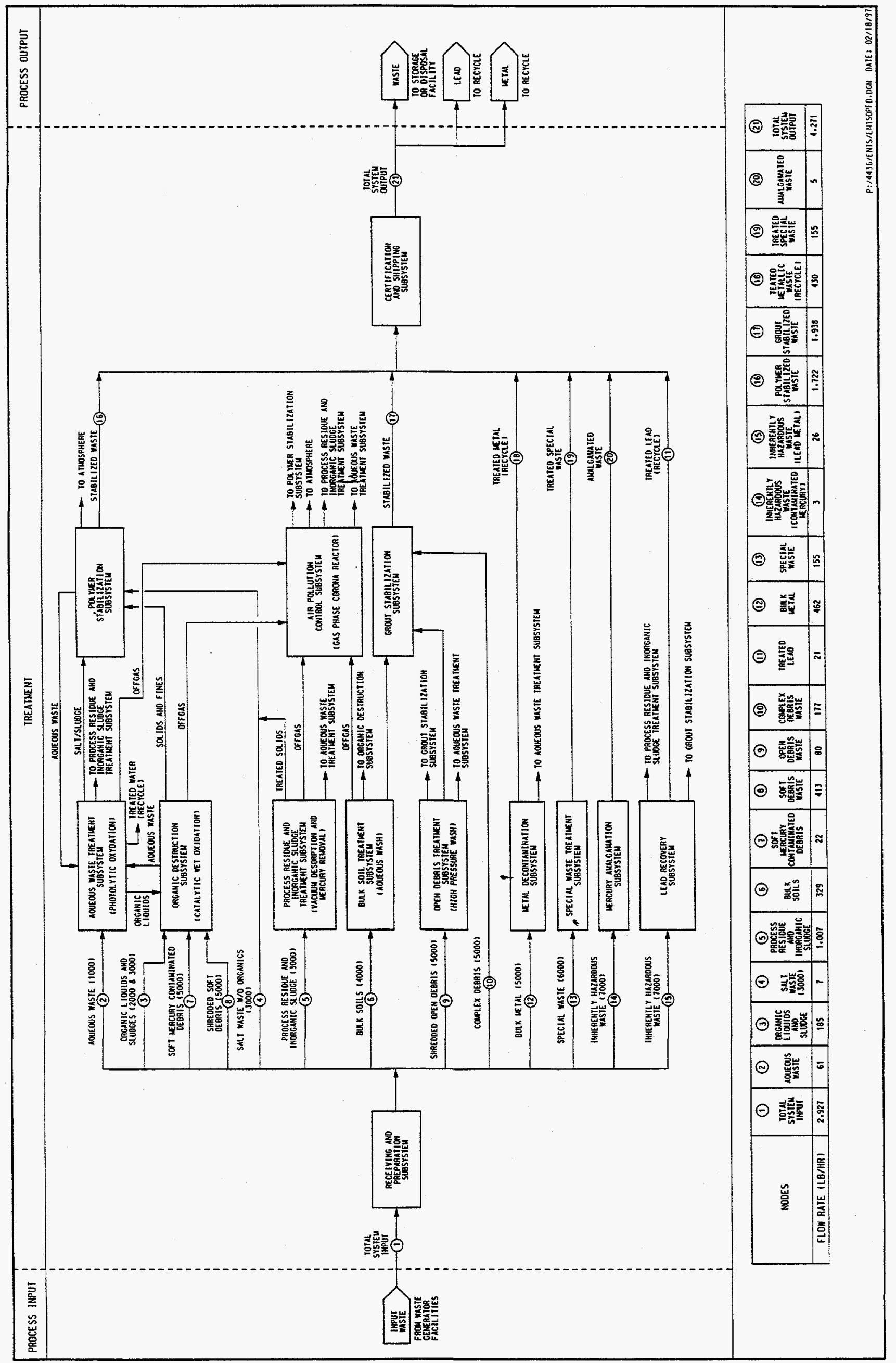

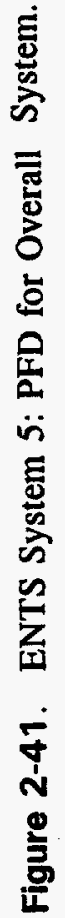




\section{SYSTEM FLOW RATES}

This section contains a summary of mass flow rates of the major inputs and outputs of the five ENTS systems and the methods used to make these calculations. This information is presented in several figures in this section. Detailed information on mass flow rates is presented in the process flow diagrams (see Appendix A).

The mass flow rate data used in this preconceptual design study are based on many assumptions. These assumptions, discussed below, must be validated before commencing more detailed design activities.

Mass flow rates for key subsystems of the five ENTS flow sheets were used to size the systems. These flow rates were calculated by modeling the flow sheets with ASPEN PLUS (ASPEN) (Aspen Technology, 1994). The ASPEN model used in the modeling of INTS System 5 was used as the basis for modeling the five ENTS systems.

\subsection{System Input Feed Rates}

As in the ITTS and INTS studies, the feed rates for the ENTS systems are uniformly calculated based on 4,032 hours of operation per year for 20 years. The calculations are based on a feed rate of 2,927 pounds per hour $(\mathrm{lb} / \mathrm{hr})$ to the system. The input to the various subsystems may vary, but the total feed rate to systems in these studies (ENTS, INTS and ITTS) is always $2,927 \mathrm{lb} / \mathrm{hr}$. The chemical composition of the input waste to each of the subsystems is identical to that of System 5 of the INTS study. Feed input rate tables are presented in Section 3 of the INTS study report. Waste input data in Table 3-1 (below) show the input waste plus major secondary waste streams that have been calculated in the mass balance studies.

\subsection{System Secondary Waste}

Secondary waste produced within the facility includes APC scrubber salts, decontamination subsystem sludge, sludge from the CWO process, and organic liquids from vacuum desorption condensed offgas.

\subsection{Key Assumptions for Mass Flow Calculations}

Detailed assumptions for mass flow calculations are presented in the INTS report. Densities and other key assumptions used in the calculations are as follows:

- Density of waste input and output (pounds per cubic foot [lb/cu.ft]):

- Input waste $=64 \mathrm{lb} / \mathrm{ft}^{3}$

- Metal $=480 \mathrm{lb} / \mathrm{ft}^{3}$

- Aqueous liquid $=62 \mathrm{lb} / \mathrm{ft}^{3}$

- $\quad$ Soil $=67 \mathrm{lb} / \mathrm{ft}^{3}$

- Polymer stabilized salts $=80 \mathrm{lb} / \mathrm{ft}^{3}$

- Polymer stabilized salts/residues/waste $=94 \mathrm{lb} / \mathrm{ft}^{3}$

- Screw compacted waste pellets $=94 \mathrm{lb} / \mathrm{ft}^{3}$

- Grout stabilized waste $=126.6 \mathrm{lb} / \mathrm{ft}^{3}$

- Stabilized mercury amalgam $=733 \mathrm{lb} / \mathrm{ft}^{3}$ 
- Density of reagents and additives:

- Caustic $(\mathrm{NaOH})=133 \mathrm{lb} / \mathrm{ft}^{3}$

- Acid solution $=62 \mathrm{lb} / \mathrm{ft}^{3}$

- Ammonia $=45 \mathrm{lb} / \mathrm{ft}^{3}$

- Nitric acid $=94 \mathrm{lb} / \mathrm{ft}^{3}$

- Silver nitrate $=271 \mathrm{lb} / \mathrm{ft}^{3}$

- Hydrogen peroxide $=91 \mathrm{lb} / \mathrm{ft}^{3}$

- Polyethylene $=80 \mathrm{lb} / \mathrm{ft}^{3}$

- $\quad$ Clay $=80 \mathrm{lb} / \mathrm{ft}^{3}$

- Copper for mercury amalgamation $=557 \mathrm{lb} / \mathrm{ft}^{3}$

- Mercury content:

- Elemental mercury (100 percent $\mathrm{Hg}$ ) comprises 5 percent of the waste designated as mercury-contaminated.

- Mercury concentration in the remaining 95 percent of the mercury-contaminated stream is 5 percent.

- Stabilization formulas (all on a mass basis):

- Polymer = One part polymer to one part waste.

- Grout = Two parts grout to one part waste.

- Polymer/Pellet $=0.35$ parts polymer to one part pellets

- $\quad$ Pellets = One part additive to one part waste

\subsection{System Output}

Output calculations are presented for the five ENTS systems, allowing comparison of the offgas volumes and waste volume reduction for the different systems. System offgas, water and metal recycling outputs are presented in Table 3-2. Waste volume outputs from the five ENTS systems are presented in Table 3-3.

\subsection{Energy Balance Calculation Results}

Energy requirements for ENTS Systems 1 through 4 were calculated using ASPEN. These results are presented in Table 3-4.

\subsection{Mass Balance Calculation Results}

Simplified block diagrams showing the key system input and output products from the five ENTS systems are shown in Figures 3-1 through 3-5. The major input and output products of the subsystems are shown in the figures. These simplified figures do not include additives or all secondary streams and therefore do not contain the complete numerical balance. Detailed mass balances that were generated using ASPEN are presented in Table 3-5. Additional mass balance information is presented in the PFDs presented in Section 2. A comparison of the mass balance results with INTS and ITTS systems is presented in Section 5. 
Table 3-1. Input feed rates to ENTS subsystems (lb/hr).

\begin{tabular}{|l|c|c|c|c|c|}
\hline Subsystem $^{\mathrm{a}}$ & $\begin{array}{c}\text { ENTS } \\
\text { System 1 }\end{array}$ & $\begin{array}{c}\text { ENTS } \\
\text { System 2 }\end{array}$ & $\begin{array}{c}\text { ENTS } \\
\text { System 3 }\end{array}$ & $\begin{array}{c}\text { ENTS } \\
\text { System 4 }\end{array}$ & $\begin{array}{c}\text { ENTS } \\
\text { System 5 }\end{array}$ \\
\hline Administration & 2,927 & 2,927 & 2,927 & 2,927 & 2,927 \\
\hline Receiving and preparation & 2,927 & 2,927 & 2,927 & 2,927 & 2,927 \\
\hline Aqueous waste treatment & 1,945 & 1,653 & 1,914 & 1,914 & 1,956 \\
\hline Organic destruction & 256 & 233 & 256 & 256 & 256 \\
\hline $\begin{array}{l}\text { Process residue and inorganic } \\
\text { sludge treatment }\end{array}$ & 1,007 & & 1,007 & 1,007 & 1,020 \\
\hline Bulk soil treatment & 329 & 329 & 329 & 329 & 329 \\
\hline Soft debris treatment & 435 & 435 & 435 & 435 & 435 \\
\hline Open debris treatment & 80 & 80 & 80 & 80 & 80 \\
\hline Complex debris treatment & 177 & 177 & & & \\
\hline Metal decontamination & 462 & 462 & 462 & 462 & 462 \\
\hline Mercury amalgamation & 4.0 & 3.8 & 2.7 & 2.7 & 2.7 \\
\hline Lead recovery & 26 & 26 & 26 & 26 & 26 \\
\hline Polymer stabilization & 1,128 & 176 & 1,772 & & 1,127 \\
\hline Claytec Process & Not Used & Not Used & Not Used & 1,772 & Not Used \\
\hline Air pollution control & 1,507 & 1,731 & 1,433 & 1,433 & 1,433 \\
\hline Vitrification & 495 & 1,502 & Not Used & Not Used & Not Used \\
\hline Grout Stabilization & Not Used & Not Used & Not Used & Not Used & 650 \\
\hline Special treatment & 155 & 155 & 155 & 155 & 155 \\
\hline Certification and shinning & 3,222 & 2,701 & 3687 & 4762 & 4,271 \\
\hline
\end{tabular}

Notes:

(a) Input to all subsystems includes only the primary and the secondary waste streams

(b) Treated by vitrification subsystem

(c) Includes mercury contaminated complex debris.

(d) Complex debris is treated by polymer stabilization

(e) Complex debris is treated by grout stabilization

(f) Claytec and polymer macro-encapsulation are used in ENTS-4 
Table 3-2. Offgas, water and metal recycle outputs from the five ENTS systems (lb/hr).

\begin{tabular}{|l|r|r|r|r|r|}
\hline Subsystem & \multicolumn{1}{|c|}{$\begin{array}{c}\text { ENTS } \\
\text { System 1 }\end{array}$} & $\begin{array}{c}\text { ENTS } \\
\text { System 2 }\end{array}$ & $\begin{array}{c}\text { ENTS } \\
\text { System 3 }\end{array}$ & $\begin{array}{c}\text { ENTS } \\
\text { System 4 }\end{array}$ & $\begin{array}{c}\text { ENTS } \\
\text { System 5 }\end{array}$ \\
\hline Offgas from APC & 1703.493 & 2,541 & 1,433 & 1,433 & 1,433 \\
\hline Water Discharge & 1,262 & 941 & 1,233 & 1,233 & 564 \\
\hline Metal/ lead Recycle & 605 & 605 & 456 & 456 & 451 \\
\hline
\end{tabular}


Table 3-3. Waste volume outputs from the five ENTS systems $\left(\mathrm{ft}^{3} / \mathrm{hr}\right)$

\begin{tabular}{|l|c|c|c|c|c|}
\hline Subsystem & $\begin{array}{c}\text { ENTS } \\
\text { System 1 }\end{array}$ & $\begin{array}{c}\text { ENTS } \\
\text { System 2 }\end{array}$ & $\begin{array}{c}\text { ENTS } \\
\text { System 3 }\end{array}$ & $\begin{array}{c}\text { ENTS } \\
\text { System 4 }\end{array}$ & $\begin{array}{c}\text { ENTS } \\
\text { System 5 }\end{array}$ \\
\hline \hline Polymer stabilization & 18.3 & 3.7 & 32.7 & - & 18.3 \\
\hline Claytec & - & - & - & 44.1 & - \\
\hline Vitrification & 3.9 & 8.5 & - & - & - \\
\hline Grout stabilization & - & - & - & - & 15.3 \\
\hline $\begin{array}{l}\text { Mercury } \\
\text { amalgamation }\end{array}$ & 0.01 & 0.01 & 0.01 & 0.01 & 0.01 \\
\hline $\begin{array}{l}\text { Metal to recycle } \\
\text { Special waste }\end{array}$ & 1.3 & 1.3 & 1.0 & 1.0 & 0.9 \\
\hline \hline $\begin{array}{l}\text { All subsystems waste } \\
\text { to disposal }\end{array}$ & 24.7 & 2.4 & 2.4 & 2.4 & 2.4 \\
\hline \hline $\begin{array}{l}\text { Volume reduction } \\
\text { factor (vol in/vol out) }\end{array}$ & 1.82 & 14.6 & 35.1 & 46.5 & 36.0 \\
\hline
\end{tabular}


Table 3-4. Energy Requirement for ENTS Systems (BTU/hr)

\begin{tabular}{|c|c|c|c|c|c|c|c|}
\hline Subsystem & Unit Operation & Temperature (F) & Energy Type & $\begin{array}{c}\text { System } 1 \\
\text { Vitrification }\end{array}$ & $\begin{array}{c}\text { System 2 } \\
\text { Vitrification }\end{array}$ & $\begin{array}{l}\text { System 3 } \\
\text { Polymer }\end{array}$ & $\begin{array}{l}\text { System } 4 \\
\text { Claytec }\end{array}$ \\
\hline \multirow[t]{4}{*}{ APC } & GPCR temp. & & electricity & 1,268 & 2,237 & 404 & 408 \\
\hline & GPCR power & & electricity & 50,162 & 105,244 & 39,450 & 39,448 \\
\hline & scrubber & 100 & cooling water & $(481,764)$ & $(2,230,000)$ & $(99,031)$ & $(98,898)$ \\
\hline & HEPA heater & 300 & electricity & 78,163 & 121,382 & 63.794 & 63,788 \\
\hline \multirow[t]{4}{*}{ Aqueous Waste Treatment } & $\mathrm{OH}$ generator & 100 & electricity & 2,801 & $(4,700)$ & 3,534 & 3,539 \\
\hline & Photolytic UV & 100 & cooling water & $(22,604)$ & $(21,671)$ & $(22,618)$ & $(22,621)$ \\
\hline & scrubber & 100 & cooling water & $(22,063)$ & $(6,761)$ & $(22,126)$ & $(22,126)$ \\
\hline & concentrator & 150 & natural gas & 158 & 95 & 112 & 112 \\
\hline Bulk Soil & drver & 200 & natural gas & 54,566 & 54,566 & 54,566 & 54,566 \\
\hline Ha Amaloamation & reactor & 2.000 & electricity & 1.102 & 1.062 & 751 & 1.102 \\
\hline \multirow[t]{3}{*}{ Organic Destruction } & CWO & 302 & cooling water & $(3,653,274)$ & $(3,192,410)$ & $(3,653,269)$ & $(3,662,835)$ \\
\hline & equillbration & 302 & c. water, elect if $>0$ & $(11,337)$ & 989 & $(11,337)$ & $(11,776)$ \\
\hline & scrubber & 100 & cooling waler & $(351,250)$ & $(315,217)$ & $(351,250)$ & $(352,204)$ \\
\hline Pb Melter & melter & 2.000 & natural gas & 1.602 & 1602 & 1.602 & 1.602 \\
\hline Polvmer & drver & 200 & nat gas.cw if $<0$ & 16.130 & $(1,914)$ & 73.768 & 73.766 \\
\hline \multirow[t]{6}{*}{ Debris } & vacuum desorp. & 550 & natural gas & 499,722 & & 499,722 & 499,722 \\
\hline & CWO & 302 & cooling water & $(4,625,297)$ & $(4,625,308)$ & $(4,625,297)$ & $(4,625,297)$ \\
\hline & equilibration & 302 & electricily & 808 & 804 & 808 & 808 \\
\hline & scrubber & 100 & cooling water & $(388,142)$ & $(387,732)$ & $(387,732)$ & $(387,732)$ \\
\hline & metal melter & 3,000 & electricity & 516,151 & 516,151 & & \\
\hline & Hg retort & 500 & natural gas & 31.951 & 31,951 & & 31.951 \\
\hline \multirow[t]{3}{*}{ Vitrification } & vitrifier & 3,000 & lelectricity & $1,918,000$ & $2,430,000$ & & \\
\hline & & $\begin{array}{l}\text { Total Energy Consumption } \\
\text { (BTU) }\end{array}$ & \begin{tabular}{|l|} 
electricity \\
natural gas \\
cooling water \\
\end{tabular} & \begin{tabular}{r|}
$2,567,187$ \\
604,129 \\
$(9,555,731)$ \\
\end{tabular} & $\begin{array}{r}3,170,932 \\
88,214 \\
(10,781,013) \\
\end{array}$ & \begin{tabular}{r|}
108,337 \\
629,770 \\
$(9,172,660)$ \\
\end{tabular} & $\begin{array}{r}108,685 \\
661,719 \\
(9,183,489) \\
\end{array}$ \\
\hline & & Hourly Energy Costs (\$/hr) & \begin{tabular}{|l|} 
electricity unit cost $=\$ 0.05 / \mathrm{k}$ \\
gas unit cost $=\$ 2.00 / \mathrm{MMB}$ Btu \\
Total Hourly Energy Costs $(\$ /$ \\
\end{tabular} & \begin{tabular}{r|}
38 \\
1 \\
39 \\
\end{tabular} & \begin{tabular}{r|}
46 \\
0 \\
47
\end{tabular} & \begin{tabular}{l|l}
2 & \\
& \\
\end{tabular} & $\begin{array}{l}2 \\
1 \\
3\end{array}$ \\
\hline
\end{tabular}

NOTE:

1. Energy balances presented are based on $2927 \mathrm{lb} / \mathrm{hr}$ of waste input.

2. Energy balance for ENTS System 5 is the same as for INTS System 5 . 
Table 3-5. ASPEN Mass Balance Data

\begin{tabular}{|c|c|c|c|c|c|}
\hline SUBSYSTEM & $\begin{array}{c}\text { System } 1 \\
\text { Vitrification }\end{array}$ & $\begin{array}{c}\text { System } 2 \\
\text { Vitrification }\end{array}$ & $\begin{array}{l}\text { System } 3 \\
\text { Polymer }\end{array}$ & $\begin{array}{c}\text { System } 4 \\
\text { Claytec }\end{array}$ & $\begin{array}{c}\text { System } 5 \\
\text { INTS }\end{array}$ \\
\hline \multicolumn{6}{|c|}{ SUBSYSTEM INPUTS FROM GENERATOR } \\
\hline Aqueous Waste $\quad(1000)$ & 60.8 & 60.8 & 60.8 & 60.8 & 60.8 \\
\hline Organic Liquids, sludges $(2000 / 3000)$ & 185.4 & 185.4 & 185.4 & 185.4 & 185.4 \\
\hline Residue, Inorg. Sludge $(3000)$ & 1007.2 & 1007.2 & 1007.2 & 1007.2 & 1007.2 \\
\hline Salt Waste $\quad(3000)$ & 6.6 & 6.6 & 6.6 & 6.6 & 6.6 \\
\hline Bulk Soils & 328.5 & 328.5 & 328.5 & 328.5 & 328.5 \\
\hline Debris - Soft & 434.8 & 434.8 & 434.8 & 434.8 & 434.8 \\
\hline Debris - Complex & 177.4 & 177.4 & 177.4 & 177.4 & 177.4 \\
\hline Debris - Open & 80.4 & 80.4 & 80.4 & 80.4 & 80.4 \\
\hline Bulk Metal & 452.1 & 462.1 & 462.1 & 462.1 & 462.1 \\
\hline Special & 154.9 & 154.9 & 154.9 & 154.9 & 154.9 \\
\hline Mercury waste & 2.5 & 2.5 & 2.5 & 2.5 & 2.5 \\
\hline Lead Waste & 26.0 & 26.0 & 26.0 & 26.0 & 26.0 \\
\hline Total Input Waste & 2926.6 & 2926.6 & 2926.6 & 2926.6 & 2926.6 \\
\hline & & & & & \\
\hline
\end{tabular}

\begin{tabular}{|c|c|c|c|c|c|}
\hline TOTAL SUBSYSTEM INPUTS & & & & & \\
\hline & & & & & \\
\hline Hg Amalgamation Subsystem & & & & & \\
\hline Waste Input & & & & & \\
\hline Mercury waste $\quad(7000)$ & 2.50 & 2.50 & 2.50 & 2.50 & 2.50 \\
\hline Process inputs & & & & & \\
\hline additives (copper) & 2.76 & 2.66 & 1.85 & 1.85 & 1.85 \\
\hline Input from other subsystems & & & & & \\
\hline Hg retort - debris treatment & 1.30 & 1.30 & & & \\
\hline process residue/inorganic sludge & 0.15 & & 0.15 & 0.15 & 0.15 \\
\hline Total Subsystem Input & 6.71 & 6.46 & 4.50 & 4.50 & 4.50 \\
\hline & & & & & \\
\hline Air Pollution Control Subsystem & & & & & \\
\hline Waste input & & & & & \\
\hline Process inputs & & & & & \\
\hline Air: $\mathrm{O} 2$ & 45.60 & 264.77 & 0.00 & 0.00 & 0.00 \\
\hline N2 & 150.17 & 871.98 & 0.00 & 0.00 & 0.00 \\
\hline $\mathrm{NaOH}$ & 3.66 & 11.68 & 2.87 & 2.87 & 2.86 \\
\hline $\mathrm{H} 2 \mathrm{O}$ & 3.66 & 11.68 & 2.87 & 2.87 & 2.86 \\
\hline Input from other subsystems & & & & & \\
\hline aqueous waste treatment & 9.40 & 13.76 & 9.33 & 9.54 & 9.47 \\
\hline organic destruction - ofigases & 561.75 & 490.71 & 561.75 & 561.75 & 561.64 \\
\hline process residuefinorganic sludge & 79.87 & & 79.87 & 79.87 & 79.87 \\
\hline debris treatment & 781.80 & 781.80 & 781.80 & 781.80 & 781.80 \\
\hline bulk soil treatment & & & & & \\
\hline vitrification subsystem & 74.24 & 445.08 & & & \\
\hline Total Subsystem Input & 1710.16 & 2891.46 & 1438.49 & 1438.70 & 1438.50 \\
\hline & & & & 10 & 6 \\
\hline Aqueous Waste Treatment subsystem & & & & & \\
\hline Waste Input & & & & & \\
\hline Aqueous Waste $\quad(1000)$ & 60.80 & 60.80 & 60.80 & 60.80 & 60.80 \\
\hline Process Inputs & & & & & \\
\hline Air (for ozone generation) & 3.82 & 3.75 & 3.73 & 4.00 & 3.91 \\
\hline $\mathrm{H} 2 \mathrm{O} 2$ & 0.63 & 0.62 & 0.62 & 0.66 & 0.64 \\
\hline $\mathrm{H} 2 \mathrm{O}$ & 8.97 & 1.30 & 8.97 & 8.97 & 8.97 \\
\hline $\mathrm{NaOH}$ & 8.97 & 1.30 & 8.97 & 8.97 & 8.97 \\
\hline Input from other subsystems & & & & & \\
\hline APC-moisture & & 328.90 & & & \\
\hline APC - concentrate & 6.67 & 21.26 & 5.22 & 5.22 & 5.22 \\
\hline process residue/inorganic sludge & 336.57 & & 336.57 & 336.57 & 349.57 \\
\hline debris treatment & 406.89 & 406.89 & 406.89 & 406.89 & 406.89 \\
\hline bulk soil treatment & 355.35 & 355.35 & 355.35 & 355.35 & 355.35 \\
\hline polymer stabilization & 265.73 & & 235.97 & 235.97 & 265.50 \\
\hline metal decontamination & 126.18 & 126.18 & 126.18 & 126.18 & 126.18 \\
\hline organic destruction - liquids & 386.73 & 353.43 & 386.73 & 386.73 & 386.15 \\
\hline Total Subsystem Input & 1967.29 & 1659.77 & 1935.98 & 1936.29 & 1978.13 \\
\hline & & & & & \\
\hline Process Residue and tnorganic Sludge Treatment Sub & & & & & \\
\hline Waste Input & & & & & \\
\hline Residue, Inorg. Sludge $\quad(3000)$ & 1007.20 & & 1007.20 & 1007.20 & 1007.20 \\
\hline Process inputs & & & & & \\
\hline $\mathrm{H} 2 \mathrm{O}$ & 306.00 & & 306.00 & 306.00 & 306.00 \\
\hline N2 sweep gas & 79.87 & & 79.87 & 79.87 & 79.87 \\
\hline Inpur from other subsyszems & & & & & \\
\hline $\mathrm{Pb}$ recovery & 0.01 & & 0.01 & 0.01 & 13.01 \\
\hline Total Subsystem Input & 1393.08 & & 1393.08 & 1393.08 & 1406.08 \\
\hline
\end{tabular}


Table 3-5. (continued) ASPEN Mass Balance Data

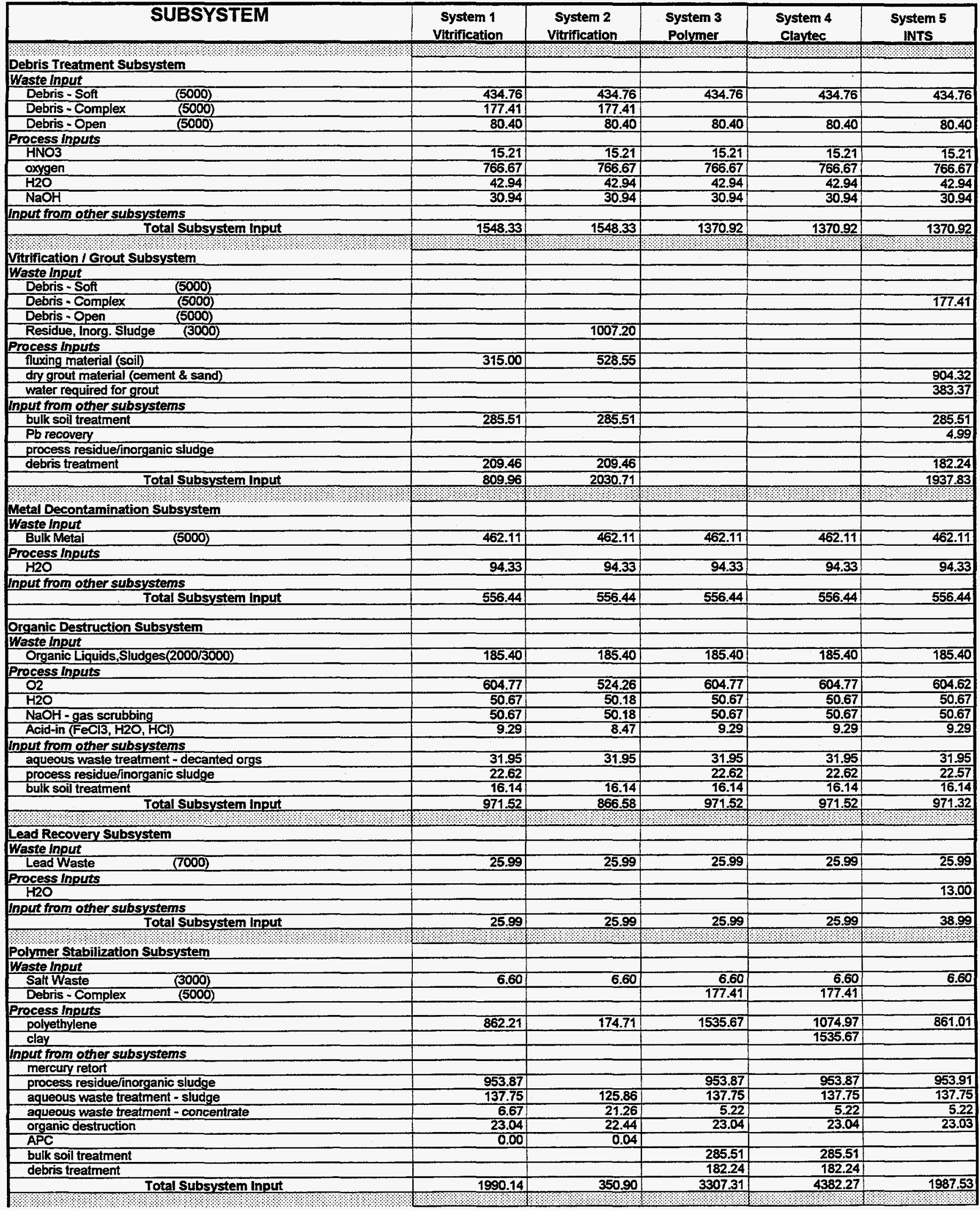


Table 3-5. (continued) ASPEN Mass Balance Data

\begin{tabular}{|c|c|c|c|c|c|}
\hline SUBSYSTEM & $\begin{array}{c}\text { System } 1 \\
\text { Vitrification }\end{array}$ & $\begin{array}{c}\text { System } 2 \\
\text { Vitrification }\end{array}$ & $\begin{array}{l}\text { System } 3 \\
\text { Polvmer }\end{array}$ & $\begin{array}{c}\text { System } 4 \\
\text { Claytec }\end{array}$ & $\begin{array}{c}\text { System } 5 \\
\text { INTS }\end{array}$ \\
\hline \multicolumn{6}{|l|}{ Bulk Soll Treatment Subsystem } \\
\hline \multicolumn{6}{|l|}{ Waste input } \\
\hline Bulk Solls & 328.50 & 328.50 & 328.50 & 328.50 & 328.50 \\
\hline \multicolumn{6}{|l|}{ Process inputs } \\
\hline $\mathrm{H} 2 \mathrm{O}$ & 328.50 & 328.50 & 328.50 & 328.50 & 328.50 \\
\hline \multicolumn{6}{|l|}{ Input from other subsystems } \\
\hline Total Subsystem Input & 657.00 & 657.00 & 657.00 & 657.00 & 657.00 \\
\hline & & & & से & \\
\hline \multicolumn{6}{|l|}{ Special Waste Treatment Subsystem } \\
\hline \multicolumn{6}{|l|}{ Waste Input } \\
\hline Special & 154.90 & 154.90 & 154.90 & 154.90 & 154.90 \\
\hline \multicolumn{6}{|l|}{ Process inputs } \\
\hline \multicolumn{6}{|l|}{ Input from other subsystems } \\
\hline Total Subsystem Input & 154.90 & 154.90 & 154.90 & 154.90 & 154.90 \\
\hline 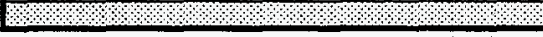 & & & & & \\
\hline SUBSYSTEM OUTPU & & & & & \\
\hline & & & $\underline{1}$ & & \\
\hline Hg Amalgamation subsystem & & & & & \\
\hline Process Qutputs & 6.71 & 6.46 & 4.50 & 4.50 & 4.50 \\
\hline Output to other subsystems & & & & & \\
\hline Total Subsystem Output & 6.71 & 6.46 & 4.50 & 4.50 & 4.50 \\
\hline & & 3 & 3 & & \\
\hline Air Pollution Control Subsystem & & & & & \\
\hline Process Outputs & & & & & \\
\hline Ofigas & & & & & \\
\hline $\mathrm{O} 2$ & 37.95 & 146.73 & 30.84 & 30.84 & 30.85 \\
\hline N2 & 234.31 & 876.19 & 84.07 & 84.28 & 84.21 \\
\hline NO & 0.67 & 0.67 & 0.67 & 0.67 & 0.67 \\
\hline $\mathrm{H} 2 \mathrm{O}$ & 37.13 & 85.18 & 3.79 & 3.78 & 3.77 \\
\hline $\mathrm{CO} 2$ & 1391.10 & 1427.73 & 1313.75 & 1313.75 & 1313.64 \\
\hline total oficas & 1703.49 & 2541.32 & 1433.27 & 1433.48 & 1433.29 \\
\hline Output to other subsystems & $\therefore$ & & & & \\
\hline aqueous waste treatment - salts & 6.67 & 21.26 & $5 . \overline{22}$ & 5.22 & 5.22 \\
\hline aqueous waste treatment - moisture & & 328.90 & & & \\
\hline polymer stabilization - metals & 0.00 & 0.04 & & & \\
\hline Total Subsystem Output & 1710.17 & 2891.52 & 1438.49 & 1438.70 & 1438.50 \\
\hline & +3 & 3 & 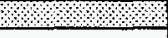 & +1 & $2+3$ \\
\hline Aqueous Waste Treatment Subsystem & & & & & \\
\hline Process Outputs & & & & & \\
\hline aqueous recycle & 1781.80 & 1468.78 & 1752.01 & 1752.11 & 1794.53 \\
\hline Output to other subsystems & & & & & \\
\hline organic destruction - decanted orgs & 31.95 & 31.95 & 31.95 & 31.95 & 31.95 \\
\hline polymer stabilization & 137.75 & 125.86 & 137.75 & 137.75 & 137.75 \\
\hline polymer stabilization - concentrate & 6.67 & 21.26 & 5.22 & 5.22 & 5.22 \\
\hline$A P C$ & 9.40 & 13.76 & 9.33 & 9.54 & 9.47 \\
\hline Total Subsystem Output & 1967.57 & 1661.60 & 1936.26 & 1936.57 & 1978.91 \\
\hline & 0 & $\$$ & 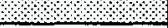 & $\$$ & 3 \\
\hline Process Residue and Inorganic Sludge Treatm & & & & & \\
\hline Process Outputs & & & & & \\
\hline Output to other subsystems & & & & & \\
\hline polymer stabilization & 953.87 & & 953.87 & 953.87 & 953.91 \\
\hline organic destruction & 22.62 & & 22.62 & 22.62 & 22.57 \\
\hline aqueous waste treatment & 336.57 & & 336.57 & 336.57 & 349.57 \\
\hline APC & 79.87 & & 79.87 & 79.87 & 79.87 \\
\hline grout stabilization & & & & & \\
\hline Hg amalgamation & & & & & 0.15 \\
\hline Total Subsystem Output & 1392.93 & & 1392.93 & 1392.93 & 1406.08 \\
\hline & 3 & & $0+1$ & मे & $\$$ \\
\hline Debris Treatment Subsystem & & & & & \\
\hline Process Outouts & & & & & \\
\hline metal & 148.89 & 148.89 & & & \\
\hline Output to other subsystems & & & & & \\
\hline aqueous waste treatment & 406.89 & 406.89 & 406.89 & 406.89 & 406.89 \\
\hline$A P C$ & 781.80 & 781.80 & 781.80 & 781.80 & 781.80 \\
\hline Hg amalgamation & 1.30 & 1.30 & & & \\
\hline vitrification & 209.46 & 209.46 & & & \\
\hline polymer stabilization & & & 182.24 & 182.24 & \\
\hline output from Hg retort to vit/poly & 27.22 & 27.22 & & & \\
\hline output from HP wash to vitjpoly & 82.82 & 82.82 & 82.82 & 82.82 & 82.82 \\
\hline output from CWO to vitpoly & 99.42 & 99.42 & 99.42 & 99.42 & \\
\hline output from vac. to poly & & & & & \\
\hline output from CWO to grout & & & & & 99.42 \\
\hline Total Subsystem Output & 1548.33 & 1548.34 & 1370.92 & 1370.92 & 1370.92 \\
\hline
\end{tabular}


Table 3-5. (continued) ASPEN Mass Balance Data

\begin{tabular}{|c|c|c|c|c|c|}
\hline SUBSYSTEM & $\begin{array}{c}\text { System } 1 \\
\text { Vitrification }\end{array}$ & $\begin{array}{c}\text { System } 2 \\
\text { Vitrification }\end{array}$ & $\begin{array}{l}\text { System } 3 \\
\text { Polvmer }\end{array}$ & $\begin{array}{c}\text { System } 4 \\
\text { Claytec }\end{array}$ & $\begin{array}{l}\text { System } 5 \\
\text { INTS }\end{array}$ \\
\hline \multicolumn{6}{|l|}{ Vitrification / Grout Subsystem } \\
\hline \multicolumn{6}{|l|}{ Process Outputs } \\
\hline vitrified product & 735.72 & 1585.64 & & & \\
\hline grouted product & & & & & 1937.83 \\
\hline \multicolumn{6}{|l|}{ Output to other subsystems } \\
\hline APC & 74.24 & 445.08 & & & \\
\hline Total Subsystem Output & 809.96 & 2030.71 & & & 1937.83 \\
\hline • & +3 & 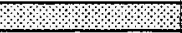 & $\therefore$ & +ి & 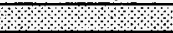 \\
\hline \multicolumn{6}{|l|}{ Metal Decontamination Subsystem } \\
\hline \multicolumn{6}{|l|}{ Process Outputs } \\
\hline decontaminated metal & 430.26 & 430.26 & 430.26 & 430.26 & 430.26 \\
\hline \multicolumn{6}{|l|}{ Output to other subsystems } \\
\hline aqueous waste treatment & 126.18 & 126.18 & 126.18 & 126.18 & 126.18 \\
\hline Total Subsystem Output & 556.44 & 556.44 & 556.44 & 556.44 & 556.44 \\
\hline \multicolumn{6}{|l|}{ Organic Destruction Subsystem } \\
\hline \multicolumn{6}{|l|}{ Process Outputs } \\
\hline \multicolumn{6}{|l|}{ Output to other subsystems } \\
\hline aqueous waste treatment - water & 386.73 & 353.43 & 386.73 & 386.73 & 386.15 \\
\hline APC-gases & 561.75 & 490.71 & 561.75 & 561.75 & 561.64 \\
\hline polymer stabilization & 23.04 & 22.44 & 23.04 & 23.04 & 23.03 \\
\hline Total Subsystem Output & 971.52 & 866.58 & 971.52 & 971.52 & 970.82 \\
\hline 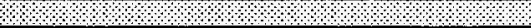 & 3 & 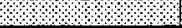 & Pl & मझ & से \\
\hline \multicolumn{6}{|l|}{ Lead Recovery Subsystem } \\
\hline \multicolumn{6}{|l|}{ Process Outputs } \\
\hline lead out & 25.99 & 25.99 & 25.99 & 25.99 & 21.00 \\
\hline \multicolumn{6}{|l|}{ Output to other subsystems } \\
\hline process residue/inorganic sludge & & & & & 13.00 \\
\hline grout - gloves & & & & & 4.99 \\
\hline Total Subsystem Output & 25.99 & 25.99 & 25.99 & 25.99 & 38.99 \\
\hline & & & 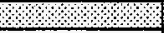 & & \\
\hline \multicolumn{6}{|l|}{ Polymer Stabilization Subsystem } \\
\hline \multicolumn{6}{|l|}{ Process Outputs } \\
\hline polymer or clay stabilized waste & 1724.41 & 349.35 & 3071.34 & 4146.30 & 1722.02 \\
\hline Output to other subsystems & & & & & \\
\hline aqueous waste treatment & 265.73 & & 235.97 & 235.97 & 265.50 \\
\hline Total Subsystem Output & 1990.14 & $\mathbf{3 4 9 . 3 5}$ & 3307.31 & 4382.27 & 1987.53 \\
\hline & 70000 & +3. & $2+3$ & 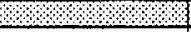 & $\$$ \\
\hline Bulk Soil Treatment Subsystem & & & & & \\
\hline Process Outputs & & & & & \\
\hline Outout to other subsystems & & & & & \\
\hline APC & & & & & \\
\hline aqueous waste treatment & 355.35 & 355.35 & 355.35 & 355.35 & 355.35 \\
\hline vitrification & 285.51 & 285.51 & & & \\
\hline grout & & & & & 285.51 \\
\hline polymer / clay stabilization & & & 285.51 & 285.51 & \\
\hline organic destruction & 16.14 & 16.14 & 16.14 & 16.14 & 16.14 \\
\hline Total Subsystem Output & 657.00 & 657.00 & 657.00 & 657.00 & 657.00 \\
\hline & & & 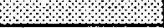 & $\lcm{1+1}$ & \\
\hline Special Waste Treatment Subsystem & & & & & \\
\hline Process Outputs & 154.90 & 154.90 & 154.90 & 154.90 & 154.90 \\
\hline Output to other subsystems & & & & & \\
\hline Total Subsystem Output & 154.90 & 154.90 & 154.90 & 154.90 & 154.90 \\
\hline Reagents input to Process (densit) & & & & & \\
\hline Cu for $\mathrm{Hg}$ amalgamation & & & & & \\
\hline $\mathrm{NaOH}$ & 94.24 & 94.10 & 93.45 & 93.45 & 93.44 \\
\hline acid solution ( $\mathrm{FeCl} 3, \mathrm{H} 2 \mathrm{O}, \mathrm{HCl})(-62)$ & 24.50 & 23.68 & 24.50 & 24.50 & 24.50 \\
\hline NH3 & & & & & \\
\hline HNO3 $(94)$ & & & & & \\
\hline $\mathrm{H} 2 \mathrm{O} 2$ & 0.63 & 0.62 & 0.62 & 0.66 & 0.64 \\
\hline grout material $\quad(126)$ & & & & & 904.32 \\
\hline water required for grout $\quad(62)$ & & & & & 383.37 \\
\hline fluxing material $\quad$ (126) & 315.00 & 528.55 & & & \\
\hline polyethytene/clay $\quad(\sim 80)$ & 862.21 & 174.71 & 1535.67 & 1535.67 & 861.01 \\
\hline vitrification fluxing agent (soil) (67) & 315.00 & 528.55 & & & \\
\hline
\end{tabular}


Table 3-5. (continued) ASPEN Mass Balance Data

\begin{tabular}{|c|c|c|c|c|c|}
\hline SUBSYSTEM & $\begin{array}{c}\text { System } 1 \\
\text { Vitrification }\end{array}$ & $\begin{array}{c}\text { System } 2 \\
\text { Vitrification }\end{array}$ & $\begin{array}{l}\text { System } 3 \\
\text { Polymer }\end{array}$ & $\begin{array}{c}\text { System } 4 \\
\text { Claytec }\end{array}$ & $\begin{array}{c}\text { System } 5 \\
\text { INTS }\end{array}$ \\
\hline \multicolumn{6}{|l|}{ Densities (Ib/f 3 ) } \\
\hline Input Waste & 64.00 & 64.00 & 64.00 & 64.00 & 64.00 \\
\hline Soil & 67.00 & 67.00 & 67.00 & 67.00 & 67.00 \\
\hline Metal (Input) & 480.00 & 480.00 & 480.00 & 480.00 & 480.00 \\
\hline Melal (Output) & 480.00 & 480.00 & 480.00 & 480.00 & 480.00 \\
\hline Aqueous Liquid & 62.00 & 62.00 & $\overline{62.00}$ & 62.00 & 62.00 \\
\hline Slag, Vitified Debris & 187.00 & 187.00 & 187.00 & 187.00 & 187.00 \\
\hline Polymerized Salt & 80.00 & 80.00 & 80.00 & 80.00 & 80.00 \\
\hline Poiymerized Ash/Metalsalt & 94.00 & 94.00 & 94.00 & 94.00 & 94.00 \\
\hline Polymerized Lime & 96.50 & 96.50 & 96.50 & 96.50 & 96.50 \\
\hline Grouted Debris & 126.60 & 126.60 & 126.60 & 126.60 & 126.60 \\
\hline Stabilized Hg Amalgam & 732.96 & 732.96 & 732.96 & 732.96 & 732.96 \\
\hline \multicolumn{6}{|l|}{ Mass Flow Rate Calculation } \\
\hline Mass Solids In (b/hr, no aqueous) & 2865.78 & 2865.78 & 2865.78 & 2865.78 & 2865.78 \\
\hline Mass Vitrified Waste Out ( $\mathrm{b} / \mathrm{hr})$ & 735.72 & 1585.64 & & & \\
\hline Mass Grouled Waste Out $(\mathrm{lb} / \mathrm{hr})$ & & & & & 1937.83 \\
\hline Mass Special Waste (not stabilized) & 154.90 & 154.90 & 154.90 & 154.90 & 154.90 \\
\hline Mass Metal \& Pb Out (lb/hr)clean & 605.14 & 605.14 & 456.25 & 456.25 & 451.26 \\
\hline Mass Polymerized Waste Out (b/hr) & 1724.41 & 349.35 & 3071.34 & 4146.30 & 1722.02 \\
\hline Mass Stabilized Hg Amalgam Out & 6.71 & 6.46 & 4.50 & 4.50 & 4.50 \\
\hline Total Mass Processed Wastes Out (b/hr, no metals) & 2621.75 & 2096.35 & 3230.74 & 4305.71 & 3819.26 \\
\hline Waste Mass Reduction Ratio (In/Out) & 1.09 & 1.37 & 0.89 & 0.67 & 0.75 \\
\hline \multicolumn{6}{|l|}{ Volumetric Flow Rate Calculation } \\
\hline Volume Solids in (ft3/hr) & 44.78 & 44.78 & 44.78 & 44.78 & 44.78 \\
\hline Volume Vitrified Waste Out (th/hr) & 3.93 & 8.48 & & & \\
\hline Volume Grouted Waste Out (ft3/hr) & & & & & 15.31 \\
\hline Volume Special Wastes (not stabilized) ( $\mathrm{f} 3 / \mathrm{hr})$ & 2.42 & 2.42 & 2.42 & 2.42 & 2.42 \\
\hline Volume Metal/Pb Out (fi3/hr) & 1.26 & 126 & 0.95 & 0.95 & 0.94 \\
\hline Volume Polymerized Waste Out (ft3/hr) & 18.34 & 3.72 & 32.67 & 44.11 & 18.32 \\
\hline Vol Stabilized Hg Amalgam Out & 0.01 & 0.01 & 0.01 & 0.01 & 0.01 \\
\hline Total Volume Solids Out to Disposal (fit $3 / \mathrm{hr}$, no metals) & 24.71 & 14.62 & 35.10 & 46.54 & 36.05 \\
\hline Volume Melal to Recycle (t $\left.3 / h^{2}\right)$ & 1.26 & 1.26 & 0.95 & 0.95 & 0.94 \\
\hline Waste Volume Reduction Ratio (In/OU) & 1.81 & 3.06 & 1.28 & 0.96 & 1.24 \\
\hline \multicolumn{6}{|l|}{\begin{tabular}{|c} 
Total Water Requirements \\
\end{tabular}} \\
\hline Aqueous Waste Treatment & & & & & 8.97 \\
\hline Inorganic Sludge Washing / Hg Leaching & & & & & 306.00 \\
\hline \multicolumn{6}{|l|}{ Lead Recovery } \\
\hline Debris Treatment & 42.94 & 42.94 & 42.94 & 42.94 & 42.94 \\
\hline Metal Decontamination & 94.33 & 94.33 & 94.33 & 94.33 & 94.33 \\
\hline Organic Destruction & 50.67 & 50.18 & 50.67 & 50.67 & 50.67 \\
\hline Soll Washing & 328.50 & 328.50 & 328.50 & 328.50 & 328.50 \\
\hline Air Pollution Control - scrubber water & 3.66 & 11.68 & 2.87 & 2.87 & 2.86 \\
\hline Water for grout ( 30 weight $\%)$ & & & & & 383.37 \\
\hline Total & 520.10 & 527.63 & 519.31 & 519.31 & 1230.64 \\
\hline Total Water Available for Recycle & 1781.80 & 1468.78 & 1752.01 & 1752.11 & 1794.53 \\
\hline Net Water Generation Rate (recycle - required) & 1261.70 & 941.15 & 1232.70 & 1232.80 & 563.89 \\
\hline
\end{tabular}




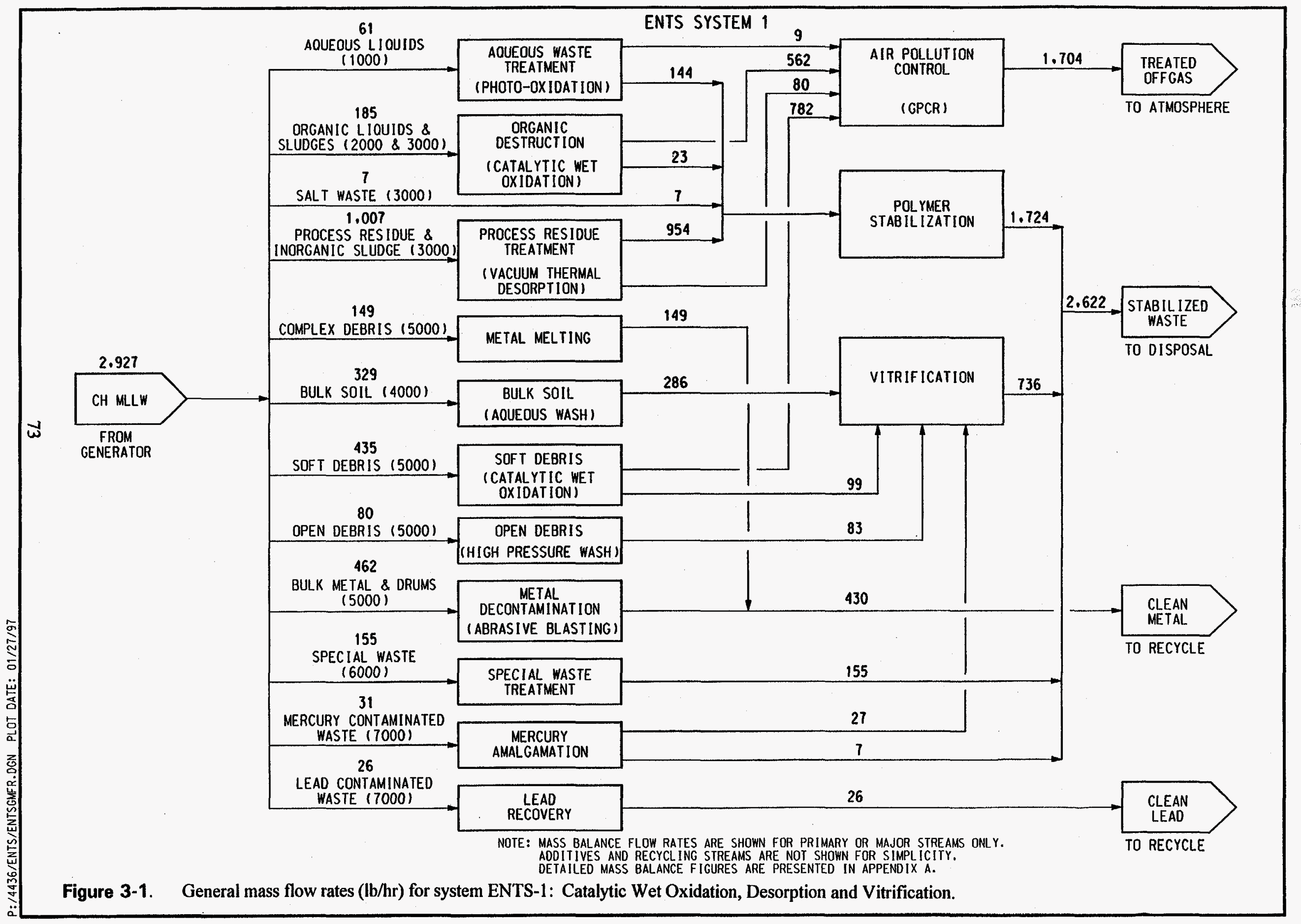




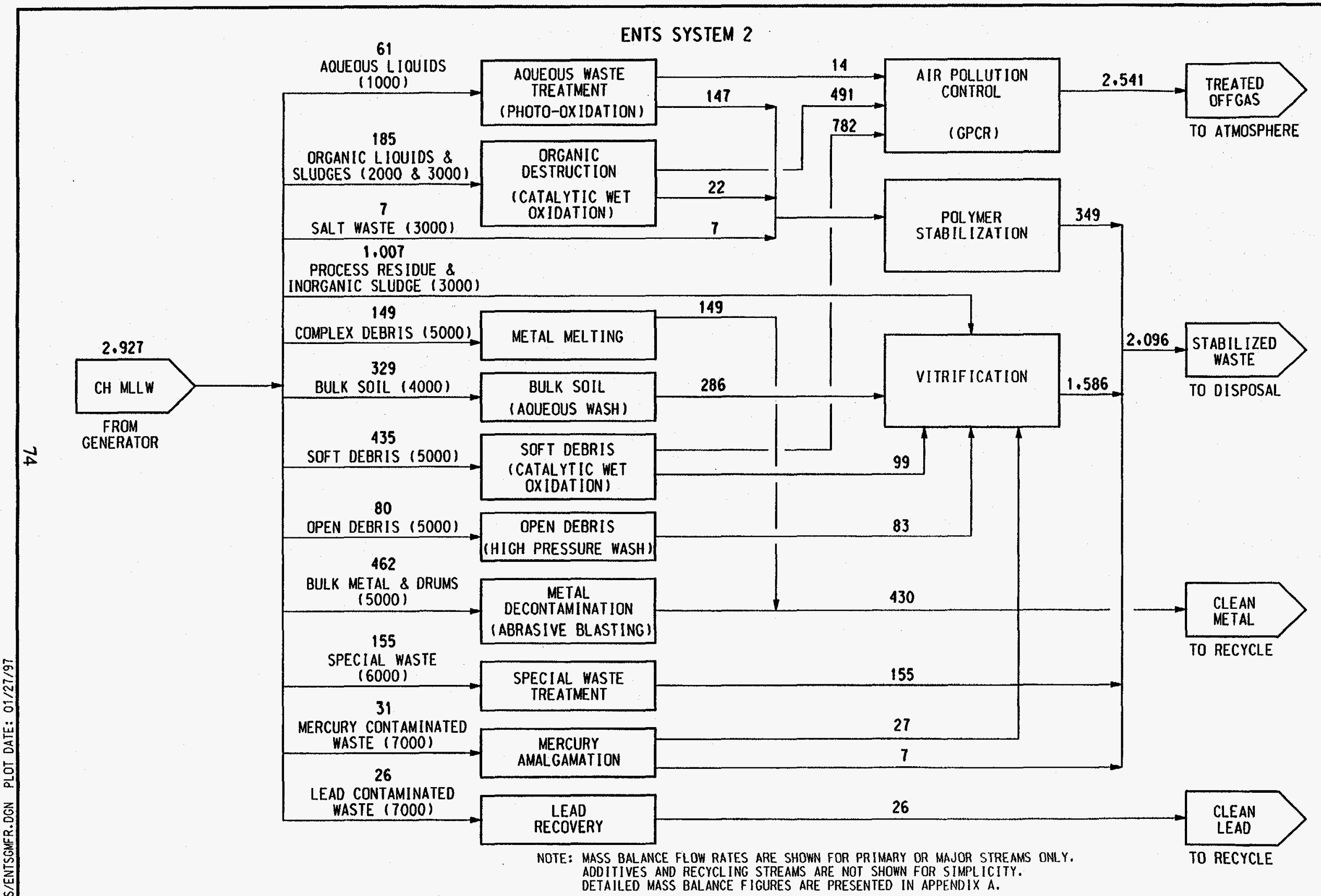

Figure 3-2. General mass flow rates (lb/hr) for system ENTS-2: Catalytic Wet Oxidation and Vitrification for Process Residues. 


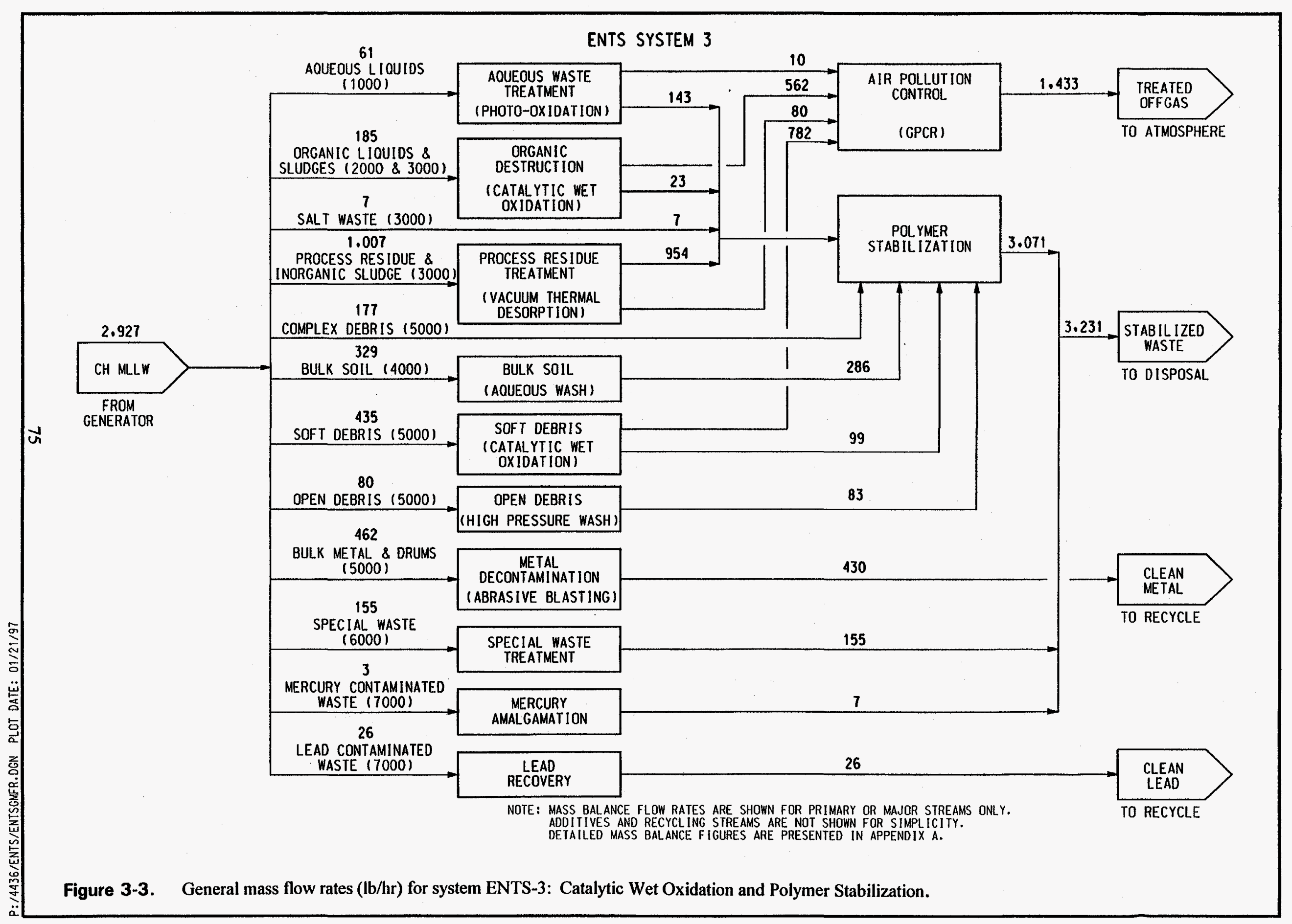




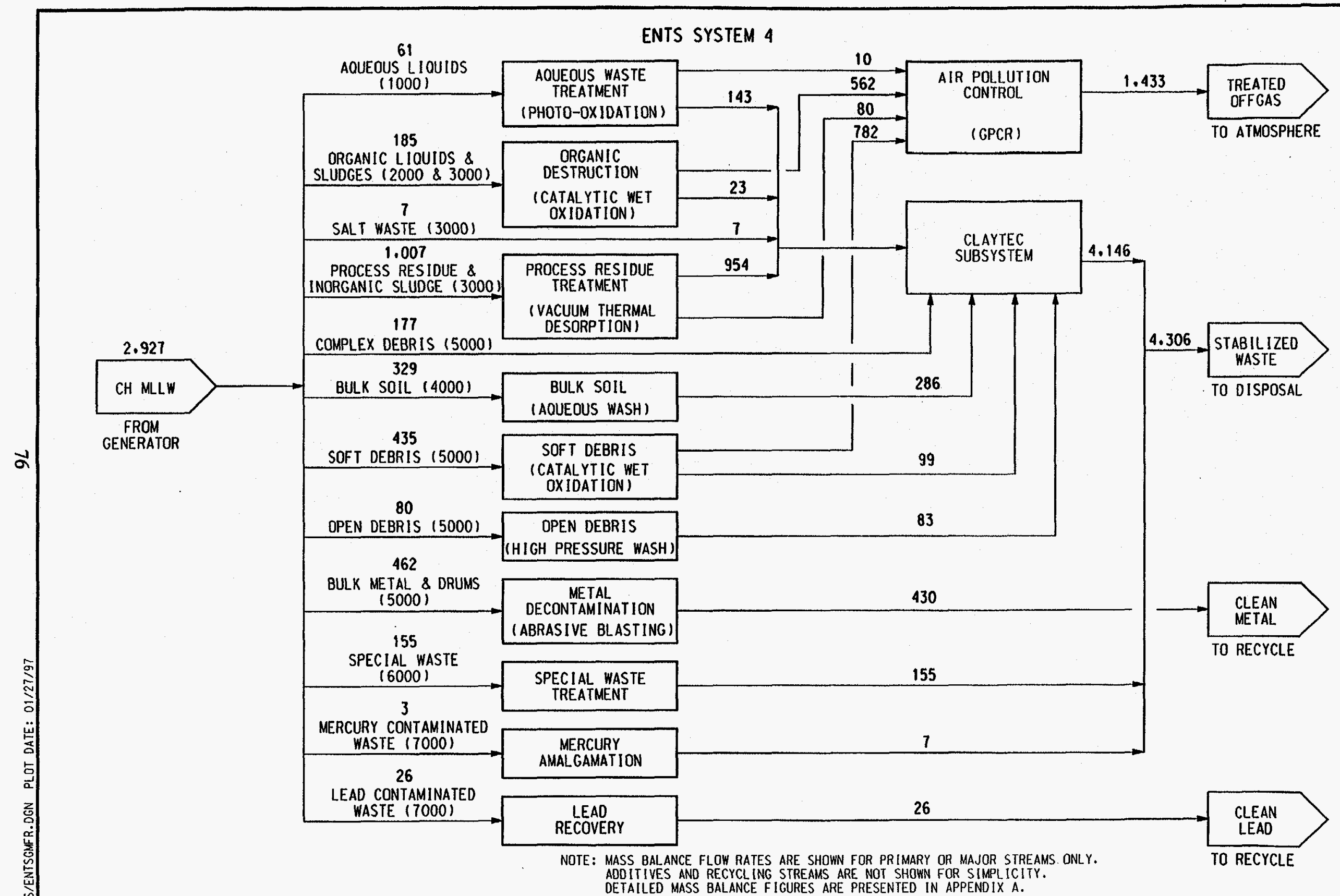

Figure 3-4. General mass flow rates (lb/hr) for system ENTS-4: Catalytic Wet Oxidation, Desorption, Compaction and Polymer Stabilization. 


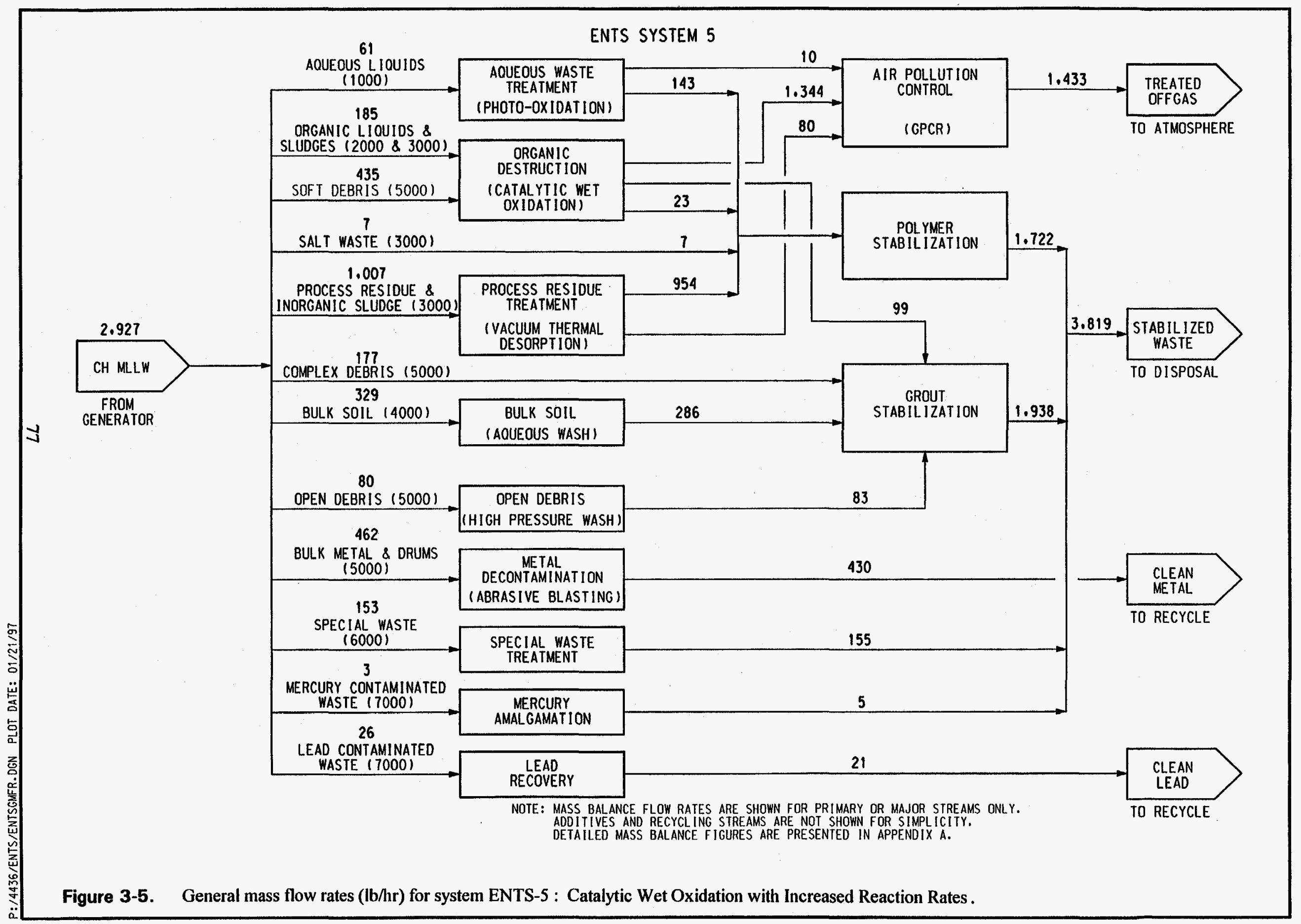




\section{TOTAL LIFE CYCLE COST ESTIMATES}

This section contains a summary of planning level Total Life Cycle Costs (TLCC) estimates for the five ENTS systems. In this report TLCC estimates are expressed as either "with disposal" or "without disposal".

\subsection{Estimating Methods, Basis, and Assumptions}

Estimating methodology and assumptions previously documented in the ITTS and INTS were used to develop the ENTS TLCC estimates. Cost estimates are presented at the end of this section.

Treatment systems reviewed in ENTS are sized at the nominal capacity of $2,927 \mathrm{lb} / \mathrm{hr}$ used in the ITTS and INTS studies to allow direct comparison. It is assumed that the facility will process both alpha and nonalpha MLLW. The sizing of the ENTS systems will permit treatment of the stored alpha and nonalpha MLLW for a 20 -year period.

\subsection{Treatment Facility TLCC (with Disposal) Estimate Summaries}

Similar to previous ITTS and INTS studies, the TLCC estimate for each facility is divided into seven component Work Breakdown Structure (WBS) elements and disposal categories. These are:

- Studies and Bench Scale Tests and Demonstration

- Demonstration Costs

- Facility Capital Costs

- Preconstruction and Preoperational Activities

- Operations and Maintenance

- Decontamination and Decommissioning

- Disposal Cost Summaries

For information on the scope of these WBS elements and cost estimating methods, the reader is referred to previously published INTS (Biagi, 1997) and ITTS (Feizollahi, 1995) study reports.

\subsection{TLCC Estimates (without Disposal) for ENTS-1, ENTS-2, ENTS-3 and ENTS-4}

TLCC estimates (without disposal) were developed and prepared using the same methodology used to develop the cost estimate for INTS-5 and are documented in Tables 4-1 through 4-4.

\subsection{TLCC (without Disposal) Estimates for ENTS-5}

The TLCC estimate for ENTS-5 includes the same WBS elements listed in Section 4.2 above. Table 4-5 presents the TLCC without disposal. The methodology used to estimate effects of increased reaction rates on TLCC are listed below. 


\subsubsection{Reaction Rate Calculations}

ENTS-5 is designed to show the effect of a ten-fold increase in reaction rate in the CWO process. Experimental reaction rate data for a variety of organic compounds was provided by the vendor (Dhooge, 1996). Table 4-11 lists the compounds, the reaction rates and the waste by category sent to the CWO process. The reaction rates are assigned to INTS waste stream categories. Two weighted average reaction rates are calculated: one for the oxidation of organic liquids and the other for soft debris. The average reaction rates are $1.6 \mathrm{lbs} / \mathrm{gal}-\mathrm{hr}$ for organic liquids, and $0.86 \mathrm{lbs} / \mathrm{gal}-\mathrm{hr}$ for soft debris. These rates were then multiplied by ten for this system.

\subsubsection{Equipment Requirements}

At ten times the reaction rate of the INTS system 5 CWO reactor, a much smaller CWO vessel is required for processing. The size of a reactor which would be able to treat both organic liquids and soft debris was calculated. The minimum reactor volume is approximately 200 gallons. However, the reactor size could be optimized to treat a single day's waste in a single shift. This would require a reactor volume of between 600 and 900 gallons. A one shift operation would provide labor and operations efficiencies, although a larger APC would be needed to treat the offgas. A 1000 gallon reactor, sized for organic liquids in INTS-5, would be able to accommodate both liquids and soft debris with this higher reaction rate with a single shift per day operation.

The soft debris and organic destruction subsystems were combined, since a single reactor and peripherals is adequate for both. Material handling, a major cost for all processes, would require additional equipment in the single subsystem. Also, the feeding system to the reactor would need to be adapted for a faster feed rate, and for both liquid and soft debris feeds. The capacity of feed preparation, filtration and neutralization equipment would be increased for single shift operation and to accommodate the waste volume of both liquids and soft debris. Costs for these items were increased from the System NT-5 baseline using the following equation (Peters and Timmerhaus, 1968):

$$
C_{2}=C_{1}\left(Q_{2} / Q_{1}\right)^{n}
$$

Cost at capacity $2\left(\mathrm{C}_{2}\right)$ equals cost at capacity $1\left(\mathrm{C}_{1}\right)$ times the ratio of equipment capacities $\left(Q_{2} / Q_{1}\right)$ taken to an exponent $n$. The capacity of the ENTS-5 CWO subsystem is the hourly capacity of both soft debris and organic destruction multiplied by 3 , because the treatment occurs in one-third the time. The cost exponent ( $\mathrm{n}$ ) is less than one, because equipment costs increase slower than capacity. For ITTS and INTS, exponents of 0.6 and 0.7 were employed respectively. The 0.7 exponent, which has been commonly used in these studies, was used in this analysis. Vendor information provided for costing the INTS organic destruction and soft debris subsystems indicated an exponent somewhat higher than 0.7 .

Cost for the reactor system using this factor also increased. Although the reactor itself would not be larger, the peripheral handling equipment would be designed to accommodate the larger mass flow rate. The equipment cost increase for a tenfold increase in reaction rate cannot be definitively predicted. A faster reaction rate will require well developed process controls. The reactor cost is increased to include either equipment or supplies (e.g. catalyst) needed to increase the reaction rate. The exact equipment needed to increase the reaction rate cannot be determined, because it is not known what kind of devices would be necessary. Therefore, the estimating method using the above capacity equation was selected.

The APC subsystem would also see an increase in capacity. These equipment costs were scaled using the combined capacity of the one shift CWO subsystem, compared to a three shift operation. 


\subsubsection{Labor Requirements}

The time and motion study presented in a separate report (Biagi, 1997) provided data on the labor requirements for the CWO subsystems. As part of this study, the labor for the improved reaction rate case was developed. The major cost of labor in both cases is related to material handling. For the increased reaction rate analysis, full time equivalent employees (FTEs) were calculated for material handling and process control. Less than four FTEs per day were required for process monitoring. Over eleven FTEs per day were necessary for material handling (15 FTEs total). The labor for ENTS-5 for this one shift operation is significantly reduced compared with NT-5 (26 FTEs for CWO combined soft debris and organic destruction subsystems).

\subsubsection{Building Requirements}

The building space for the soft debris subsystem was deleted. An additional 900 square feet for soft debris handling equipment was added to the building space for the combined CWO subsystem. The reduced building size provides reductions in both facility capital costs and decontamination and decommissioning costs.

\subsubsection{Results of the ENTS-5 Cost Analysis}

The costs for ENTS System 5 are presented in Table 4-5. The life cycle cost for a system with an order of magnitude increase in CWO reaction rates is one percent less than the INTS System 5 cost. Savings in operating labor, building and decontamination and decommissioning are offset by increases in equipment cost and maintenance labor. Research and development costs were kept the same as for INTS System 5, although additional research and development could be expected to result in the equipment and/or process by which the reaction rate would be increased.

The cost differential between NT-5 and ENTS- 5 is sensitive to the equipment costs. Maintenance equipment and labor are a function of equipment costs. As in INTS, annual maintenance equipment costs are calculated at 10 percent of the total equipment cost and annual maintenance labor is 250 percent of the maintenance equipment cost. Thus, annual costs of 35 percent of the equipment costs are incurred. Over 20 years, a $\$ 100$ increase in equipment will be accompanied by a $\$ 700$ increase in maintenance and operations. Every dollar of increase in capital cost increases eightfold over the lifetime. This is why the increases in maintenance labor and equipment for ENTS-5 organic destruction subsystem more than offset the decrease in operating labor. performed:

Because of the equipment costs effect on the subsystem costs, two sensitivity analyses were

(1) the cost exponent at 0.6 instead of 0.7

(2) the capacity of the subsystem for a three shift operation, instead of a one shift operation

The results of the first analysis are as follows:

- a 0.7 exponent in the above capacity equation yields a decrease of TLCC relative to NT-5 of 1 percent

- a 0.6 exponent yields a decrease of TLCC relative to NT -5 of 2 percent

The results of the second analysis show the following:

- processing all the waste in a single shift provides a 1 percent decrease of TLCC relative to NT-5

- processing all the waste in a three shifts provides a 3 percent decrease of TLCC relative to NT-5 
These analyses indicate that the effect of CWO reaction rates on life cycle cost is slight, if any. Decreases in the TLCC without disposal of between one and three percent over the life time of the facility are not significant. This result is not unexpected since CWO processes less than 25 percent of the waste. Adjustments to CWO costs will have little effect on the cost of processing the majority of the waste. CWO processing costs are driven by material handling which is little changed by adjusting reaction rates. Waste treated by CWO must first be received, inspected and sorted. Following CWO, the residuals will be stabilized, packaged and shipped. These costs are not affected by rate increases.

\subsection{Additional TLCC Information}

TLCC estimates with disposal are shown in Table 4-6. Unit costs are also presented on these tables. System ENTS-2 (CWO and vitrification), with the lowest volume of waste to disposal, has the lowest cost. System ENTS-4 (CWO and polymer stabilization) has the highest cost. Table 4-7 compares the TLCC with disposal by WBS element for the five ENTS systems. Operating and maintenance costs and disposal costs are the most significant discriminators between the systems.

Table 4-8 presents the normalized TLCC (without disposal) comparison, using INTS System NT-5 as the baseline. System ENTS-2 has the lowest costs. Table 4-9 presents the TLCC without disposal by subsystem. Table 4-10 presents the operating FTE Estimates for the ENTS systems and for NT-5. 
Table 4-1. TLCC (without Disposal) Estimate for ENTS-1 (in millions of dollars).

Work Breakdown Structure

CWO

Total System Cost

ENTS - 1

$(\$ 000,000)$

1.0 Studies and bench scale test costs

1.1 Manpower costs during research

$\$ 30.3$

1.2 Equipment costs

$\$ 3.2$

1.3 Installation costs

$\$ 1.2$

1.4 Project management before title I

( $10 \%$ of 1.1 through 1.3 )

$\$ 3.5$

1.5 Contingency

( $25 \%$ of 1.1 through 1.4 )

$\$ 9.5$

Subtotal 1.0

$\$ 47.7$

2.0 Demonstration costs

2.1 Manpower costs during demonstration

2.2 Design cost

$\$ 28.5$

2.3 Inspection cost

$\$ 11.5$

2.4 Project management

( $30 \%$ of 2.5 )

$\$ 2.7$

2.5 Construction cost

$\begin{array}{ll}\text { 2.5.1 } & \text { Building structure costs } \\ \text { 2.5.2 } & \text { Equipment costs } \\ \text { 2.5.3 } & \text { Indirect }\end{array}$

( $7 \%$ of 2.5 )

( $10 \%$ of 2.5 )

$\$ 3.8$

2.5 .2

Equipment costs

Indirect

( $29 \%$ of $2.5 .1 \& 2.5 .2$ )

$\$ 5.3$

$\$ 24.5$

Subtotal of 2.5

2.6 Construction management costs

2.7. Management Reserve

( $17.1 \%$ of 2.5$)$

$\$ 8.6$

$\$ 38.4$

2.8 Contingency

( $10 \%$ of 2.5 )

$\$ 6.6$

$\$ 3.8$

( $25 \%$ of 2.1 through 2.7 )

$\$ 23.9$

Subtotal 2.0

3.0 Production facility construction costs

3.1 Design cost

( $25 \%$ of 3.4 )

( $7 \%$ of 3.4 )

( $10 \%$ of 3.4 )

$\$ 119.3$

3.2 Inspection cost

$\$ 95.1$

3.3 Project management

$\$ 26.6$

3.4 Construction cost

3.4.1 Building structure costs

$\$ 38.0$

3.4.2 Equipment costs

3.4.3 Indirect

Subtotal of 3.4

3.5 Construction management

3.6 Management Reserve

3.7 Contingency Subtotal 3.0

$\$ 161.1$

$\$ 133.8$

$\$ 85.5$

$\$ 380.4$

$\$ 65.0$

( $17.1 \%$ of 3.4$)$

$\$ 38.0$

( $10 \%$ of 3.4 )

$\$ 151.3$

$\begin{array}{lll}\text { 4.0 } & \text { Operations Budget Funded Activities (See Sect. 7) } \\ & 4.1 \text { Conceptual design } & (1.5 \% \text { of } 3.0)\end{array}$

4.2 Safety assurance ( $1 \%$ of 3.0 )

$\$ 794.5$

4.3 NEPA permitting ( $\$ 6$ Mill for EIS, $\$ 1$ Mill for EA)

$\$ 11.9$

$\$ 7.9$

$\$ 7.0$

4.4 Preparation for operations $\quad(100 \%$ of 5.0$)$

$\$ 106.7$

4.5 Project Management

( $10 \%$ of 4.1 through 4.4 )

$\$ 13.4$

Subtotal 4.0

$\$ 146.9$

Total Initial Cost (1.0,2.0,3.0 \& 4.0)

$\$ 1,108.4$

5.0 Operating and maintenance costs

5.1 Annual operating costs

$\$ 56.6$

5.2 Annual utility costs

$\$ 1.9$

5.3 Annual material costs

5.4 Annual maintenance costs

$\$ 20.1$

5.5 Contingency

( $25 \%$ of 5.1 through 5.4 )

$\$ 21.3$

5.6 Subtotal 5.0

$\$ 106.7$

5.7 Total 20 year $O \& M$ cost (20 times Subtotal 5.0) 
Table 4-2. TLCC (without Disposal) Estimate for ENTS-2 (in millions of dollars).

Work Breakdown Structure

Total System Cost

ENTS - 2

$(\$ 000,000)$

$1.0 \quad$ Studies and bench scale test costs

1.1 Manpower costs during research $\$ 26.6$

$\begin{array}{lr}1.2 \text { Equipment costs } & \$ 2.7\end{array}$

1.3 Installation costs $\quad \$ 1.0$

1.4 Project management before title I $\quad(10 \%$ of 1.1 through 1.3$) \quad \$ 3.0$

1.5 Contingency $\quad(25 \%$ of 1.1 through 1.4$) \quad \$ 8.3$

Subtotal 1.0 \$41.6

$\begin{array}{lll}2.0 & \text { Demonstration casts } & \\ & 2.1 & \text { Manpower costs during demonstration }\end{array}$

2.2 Design cost $\quad(30 \%$ of 2.5$) \quad \$ 10.6$

2.3 Inspection cost $\quad(7 \%$ of 2.5$) \quad \$ 2.5$

2.4 Project management $\quad(10 \%$ of 2.5$) \quad \$ 3.5$

2.5 Construction cost 2.5.1 Building structure costs $\quad \$ 4.8$

2.5.2 Equipment costs $\$ 22.5$

2.5.3 Indirect ( $29 \%$ of $2.5 .1 \& 2.5 .2) \quad \$ 7.9$

Subtotal of 2.5

2.6 Construction management costs $\quad(17.1 \%$ of 2.5$) \quad \$ 6.0$

2.7 Management Reserve $\quad(10 \%$ of 2.5$) \quad \$ 3.5$

2.8 Contingency ( $25 \%$ of 2.1 through 2.7$) \quad \$ 21.3$

Subtotal 2.0 $\quad \$ 106.6$

3.0 Production facility construction costs

3.1 Design cost ( $25 \%$ of 3.4$)$

3.2 Inspection cost ( $7 \%$ of 3.4$)$

$\$ 91.2$

3.3 Project management $\quad(10 \%$ of 3.4$) \quad \$ 36.5$

3.4 Construction cost

$\begin{array}{lll}3.4 .1 & \text { Building structure costs } & \$ 153.5\end{array}$

3.4.2 Equipment costs $\$ 129.3$

3.4.3 Indirect $\quad(29 \%$ of $3.4 .1 \& 3.4 .2)$

Subtotal of $3.4 \quad \$ 364.8$

3.5 Construction management $\quad(17.1 \%$ of 3.4$) \quad \$ 62.4$

3.6 Management Reserve ( $\quad$ ( $10 \%$ of 3.4$) \quad \$ 36.5$

3.7 Contingency $\quad(25 \%$ of 3.1 through 3.5$) \quad \$ 145.1$

$\begin{array}{lr}\text { Subtotal } 3.0 & \$ 761.9\end{array}$

$\begin{array}{lll}4.0 & \text { Operations Budget Funded Activities (See Sect. 7) } & \\ & 4.1 \text { Conceptual design } & (1.5 \% \text { of } 3.0)\end{array}$

4.2 Safety assurance $\quad\left(\begin{array}{r}1 \% \text { of } 3.0) \\ 4.3 \mathrm{NEPA} \text { permiting }\end{array}\right.$

4.3 NEPA permitting (\$ 6 Mill for EIS, \$1 Mill for EA) $\$ 7.0$

4.4 Preparation for operations $\quad(100 \%$ of 5.0$) \quad \$ 93.9$

4.5 Project Management $\quad(10 \%$ of 4.1 through 4.4) $\quad \$ 12.0$

Subtotal 4.0 $\quad$ \$ $\$ 131.9$

Total Initial Cost $(1.0,2.0,3.0 \& 4.0) \quad S 1,042.2$

5.0 Operating and maintenance costs

5.1 Annual operating costs

$\$ 47.5$

5.2 Annual utility costs $\quad \$ 2.0$

5.3 Annual material costs $\quad \$ 6.5$

5.4 Annual maintenance costs $\quad \$ 19.2$

5.5 Contingency $\quad(25 \%$ of 5.1 through 5.4) $\quad \$ 18.8$

5.6 Subtotal 5.0 $\quad \$ 993.9$

5.7 Total 20 year $O \& M$ cost (20 times Subtotal 5.0) $\$ \$ 1,878.0$

$6.0 \quad$ Decontamination \& Decommissioning $\quad \$ 81.3$

7.0 ROM Life cvcle costs (20 years operation) 
Table 4-3. $\quad$ TLCC (without Disposal) Estimate for ENTS-3 (in millions of dollars).

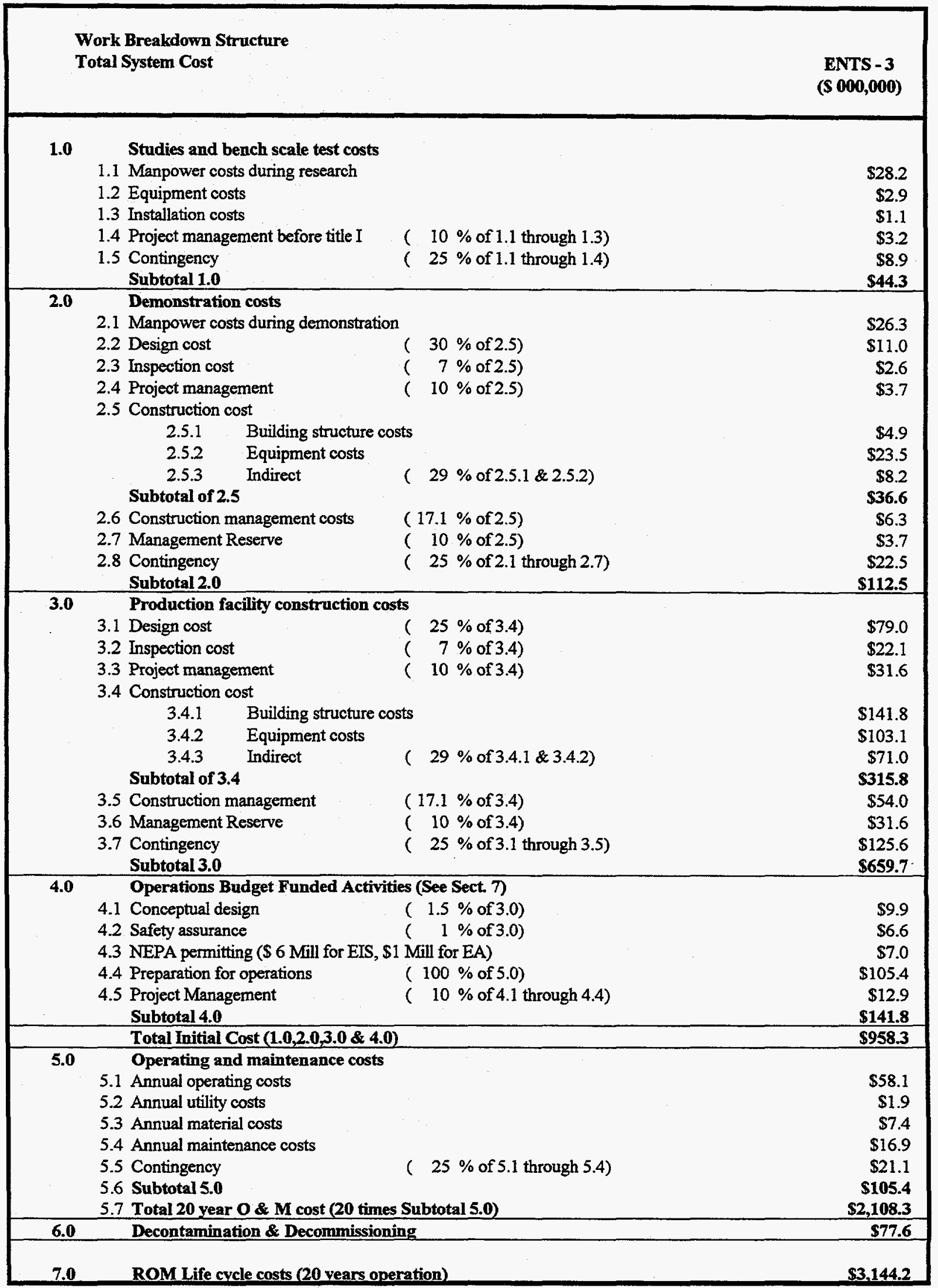


Table 4-4. TLCC (without Disposal) Estimate for ENTS-4 (in millions of dollars).

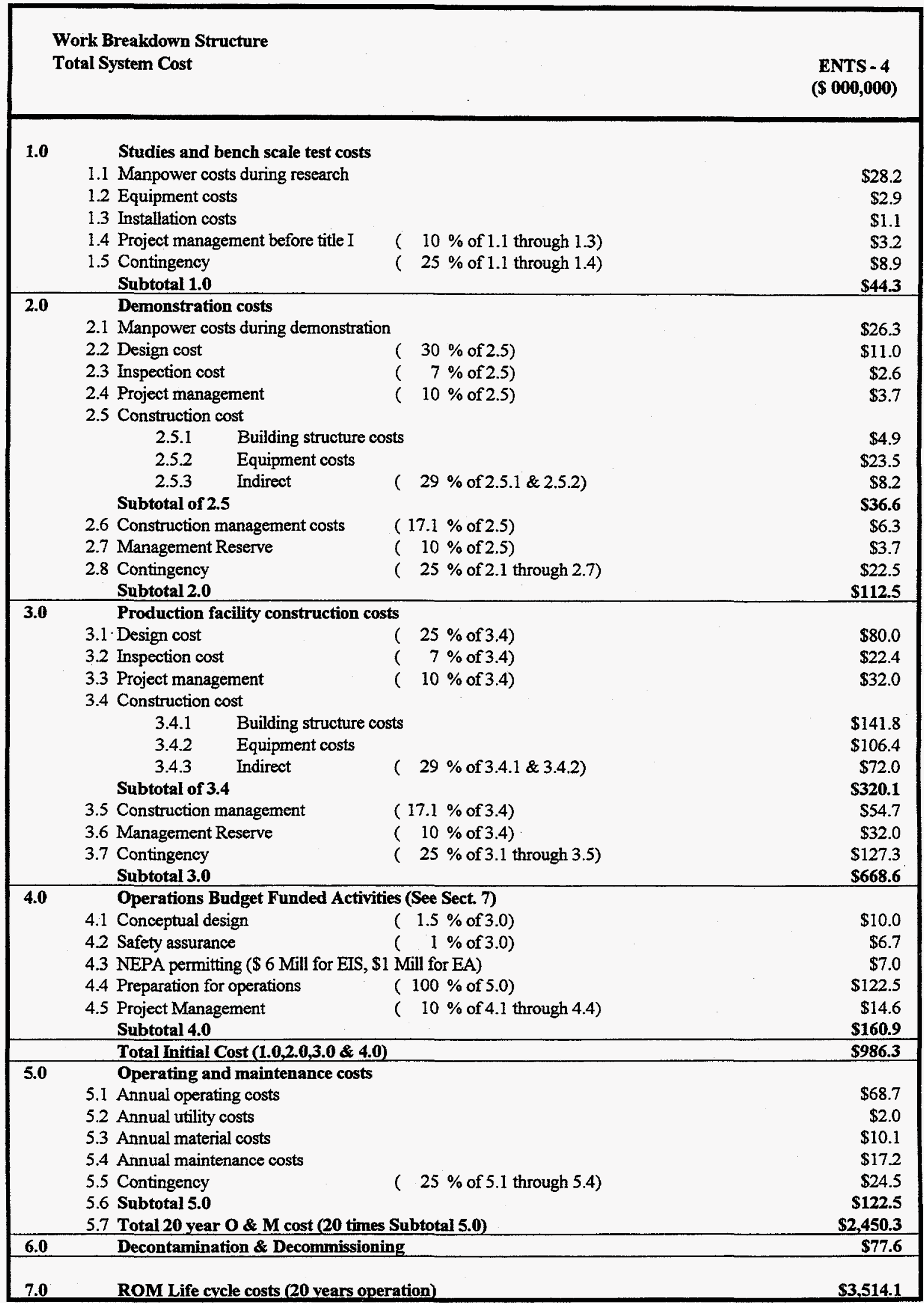

j:inellentsicostlents4.wk3 
Table 4-5. TLCC (without Disposal) Estimate for ENTS-5 (in millions of dollars).

Work Breakdown Structure

Total System Cost

ENTS 5

$(\$ 000,000)$

1.0 Studies and bench scale test costs

1.1 Manpower costs during research

1.2 Equipment costs

1.3 Installation costs

$\$ 1.2$

1.4 Project management before title I ( $10 \%$ of 1.1 through 1.3)

$\$ 3.5$

1.5 Contingency

( $25 \%$ of 1.1 through 1.4)

Subtotal 1.0

$\$ 47.7$

2.0 Demonstration costs

2.1 Manpower costs during demonstration

2.2 Design cost

2.3 Inspection cost

2.4 Project management

( $30 \%$ of 2.5 )

$\$ 28.5$

2.5 Construction cost

$\$ 11.5$

$\$ 2.7$

$\$ 3.8$

2.5.1 Building structure costs

$\$ 5.3$

2.5.2 Equipment costs

2.5.3 Indirect

$\$ 24.5$

$\$ 8.6$

Subtotal of 2.5

2.6 Construction management costs

2.7 Management Reserve

2.8 Contingency

$29 \%$ of $2.5 .1 \& 2.5 .2$ )

$\$ 38.4$

$\$ 6.6$

$\$ 3.8$

$\$ 23.9$

Subtotal 2.0

( $17.1 \%$ of 2.5$)$

( $25 \%$ of 2.1 through 2.7 )

$\$ 119.3$

3.0 Production facility construction costs

3.1 Design cost

3.2 Inspection cost

3.3 Project management

( $25 \%$ of 3.4 )

$\$ 80.7$

( $7 \%$ of 3.4 )

$\$ 22.6$

3.4 Construction cost

( $10 \%$ of 3.4 )

$\$ 32.3$

3.4.1 Building structure costs

$\$ 136.2$

3.4.2 Equipment costs

3.4.3 Indirect

( $29 \%$ of $3.4 .1 \& 3.4 .2)$

$\$ 114.2$

Subtotal of 3.4

$\$ 72.6$

$\$ 323.0$

3.5 Construction management $\quad$ ( $17.1 \%$ of 3.4)

$\$ 55.2$

3.6 Management Reserve

( $10 \%$ of 3.4)

$\$ 32.3$

3.7 Contingency

( $25 \%$ of 3.1 through 3.5 )

$\$ 128.5$

Subtotal 3.0

$\$ 674.6$

4.0 Operations Budget Funded Activities (See Sect. 7)

4.1 Conceptual design (1.5\% of 3.0)

4.2 Safety assurance (1\% of 3.0)

$\$ 10.1$

$\$ 6.7$

$\$ 7.0$

4.3 NEPA permitting ( $\$ 6$ Mill for EIS, $\$ 1$ Mill for EA)

$\$ 101.2$

4.4 Preparation for operations $\quad(100 \%$ of 5.0)

$\$ 12.5$

4.5 Project Management $\quad$ ( $10 \%$ of 4.1 through 4.4)

$\$ 137.5$

Subtotal 4.0

$\$ 979.1$

5.0 Operating and maintenance costs

5.1 Annual operating costs

$\$ 55.1$

5.2 Annual utility costs

$\$ 1.2$

5.3 Annual material costs

$\$ 6.1$

5.4 Annual maintenance costs

$\$ 18.6$

5.5 Contingency

( $25 \%$ of 5.1 through 5.4)

$\$ 20.2$

5.6 Subtotal 5.0

$\$ 101.2$

5.7 Total 20 year $O \& M$ cost (20 times Subtotal 5.0)

$\$ 2,023.3$

6.0 Decontamination \& Decommissionin

$\$ 75.6$

7.0 Life cycle costs (20 years operation)

$\$ 3,078.1$

$\mathrm{J}:$ inellents $\backslash$ cost $\backslash \mathrm{ENTS} 5$ 
Table 4-6. ENTS TLCC Estimates with Disposal.

\begin{tabular}{|c|c|c|c|c|c|c|c|}
\hline & units & $\begin{array}{c}\text { ENTS } \\
\text { System } 1 \\
\end{array}$ & $\begin{array}{c}\text { ENTS } \\
\text { System } 2 \\
\end{array}$ & $\begin{array}{c}\text { ENTS } \\
\text { System } 3 \\
\end{array}$ & $\begin{array}{c}\text { ENTS } \\
\text { System } 4 \\
\end{array}$ & $\begin{array}{c}\text { ENTS } \\
\text { System } 5 \\
\end{array}$ & $\begin{array}{c}\text { INTS } \\
\text { System } 5\end{array}$ \\
\hline Treatment Cost & $\$ / 1 b$ & $\$ 14.09$ & $\$ 12.72$ & $\$ 13.32$ & $\$ 14.89$ & $\$ 13.04$ & $\$ 13.17$ \\
\hline Output Volumetric Flow Rate & $\mathrm{ft} 3 / \mathrm{hr}$ & 24.7 & 14.6 & 35.1 & 46.5 & 36.0 & 36.04 \\
\hline Total treatment cost & millions of dollars & $\$ 3,326$ & $\$ 3,001$ & $\$ 3,144$ & $\$ 3,514$ & $\$ 3,078$ & $\$ 3,110$ \\
\hline Final Waste Volume & $\mathrm{ft} 3$ & $1,992,507$ & $1,179,357$ & $2,830,486$ & $3,752,669$ & $2,906,784$ & $2,906,266$ \\
\hline Disposal Cost & millions of dollars & $\$ 484$ & $\$ 287$ & $\$ 688$ & $\$ 912$ & $\$ 706$ & $\$ 706$ \\
\hline Total Life Cycle Cost & millions of dollars & $\$ 3,810$ & $\$ 3,288$ & $\$ 3,832$ & $\$ 4,426$ & $\$ 3,784$ & $\$ 3,816$ \\
\hline Total Life Cycle Unit Cost & $\$ / 1 b$ & $\$ 16.14$ & $\$ 13.93$ & $\$ 16.23$ & $\$ 18.75$ & $\$ 16.03$ & $\$ 16.17$ \\
\hline Disposal Unit Cost & $\$ / 1 \mathrm{~b}$ & $\$ 2.05$ & $\$ 1.21$ & $\$ 2.91$ & $\$ 3.86$ & $\$ 2.99$ & $\$ 2.99$ \\
\hline
\end{tabular}

Note: The treatment cost unit rate is calculated by dividing the treatment cost by the total waste input to the ENTS facility for 20 years of operation ( 236 million pounds). 
Table 4-7. TLCC Comparisons of the five ENTS systems (in millions of dollars).

\begin{tabular}{|c|c|c|c|c|c|c|}
\hline WBS Element Costs (a) & $\begin{array}{c}\text { ENTS } \\
\text { System } 1 \\
\end{array}$ & $\begin{array}{c}\text { ENTS } \\
\text { System } 2 \\
\end{array}$ & $\begin{array}{c}\text { ENTS } \\
\text { System } 3 \\
\end{array}$ & $\begin{array}{c}\text { ENTS } \\
\text { System } 4 \\
\end{array}$ & $\begin{array}{c}\text { ENTS } \\
\text { System } 5 \\
\end{array}$ & $\begin{array}{c}\text { INTS } \\
\text { System } 5 \\
\end{array}$ \\
\hline \multicolumn{7}{|l|}{ TREATMENT } \\
\hline Item $1.0,2.0$, and 4.0 & $\$ 314$ & $\$ 280$ & $\$ 299$ & $\$ 318$ & $\$ 305$ & $\$ 306$ \\
\hline Item 3.0 & $\$ 794$ & $\$ 762$ & $\$ 660$ & $\$ 669$ & $\$ 675$ & $\$ 668$ \\
\hline Item 5.0 & $\$ 2,133$ & $\$ 1,878$ & $\$ 2,108$ & $\$ 2,450$ & $\$ 2,023$ & $\$ 2,058$ \\
\hline Item 6.0 & $\$ 84$ & $\$ 81$ & $\$ 78$ & $\$ 78$ & $\$ 76$ & $\$ 78$ \\
\hline Total Treatment PLCC & $\$ 3,326$ & $\$ 3,001$ & $\$ 3,144$ & $\$ 3,514$ & $\$ 3,078$ & $\$ 3,110$ \\
\hline $\begin{array}{l}\text { DISPOSAL (b) } \\
\text { Disposal PLCC }\end{array}$ & $\$ 484$ & $\$ 287$ & $\$ 688$ & $\$ 912$ & $\$ 706$ & $\$ 706$ \\
\hline $\begin{array}{l}\text { TOTAL TREATMENT \& DISPOSAI } \\
\text { PLCC }\end{array}$ & $\$ 3,810$ & $\$ 3,288$ & $\$ 3,832$ & $\$ 4,426$ & $\$ 3,784$ & $\$ 3,816$ \\
\hline
\end{tabular}

(a) Work breakdown structure elements used in developing treatment PLCC are:

1 Studies and Bench Scale Tests

4 Operating Budget Funded Activities

2 Demonstration Costs

5 Operating \& Maintenance Costs

3 Production Facility Construction Costs

6 Decontamination \& Decommisioning Costs

(b) The disposal cost has been calculated using a unit disposal cost rate of $\$ 243 / \mathrm{ft}^{3}$. 
Table 4-8. Normalized Lifecycle Cost Comparison of the Five ENTS system (normalized with respect to INTS System 5) (in millions of dollars).

\begin{tabular}{|c|c|c|c|c|c|c|c|}
\hline \multirow{2}{*}{\multicolumn{2}{|c|}{ Cost Component }} & \multirow{2}{*}{$\begin{array}{c}\text { Actual Cost } \\
\text { INTS System } 5 \\
\end{array}$} & \multicolumn{5}{|c|}{ Normalized Costs } \\
\hline & & & ENTS 1 & ENTS 2 & ENTS 3 & ENTS 4 & ENTS 5 \\
\hline 1.0 & Studies and bench scale test costs & $\$ 47.7$ & 1.00 & 0.87 & 0.93 & 0.93 & 1.00 \\
\hline 2.0 & Demonstration costs & $\$ 119.3$ & 1.00 & 0.89 & 0.94 & 0.94 & 1.00 \\
\hline 3.0 & Production facility construction costs & $\$ 667.8$ & 1.19 & 1.14 & 0.99 & 1.00 & 1.01 \\
\hline 4.0 & Operations Budget Funded Activities & $\$ 139.3$ & 1.05 & 0.95 & 1.02 & 1.16 & 0.99 \\
\hline 5.0 & Operating and maintenance costs & $\$ 2,058.1$ & 1.04 & 0.91 & 1.02 & 1.19 & 0.98 \\
\hline 6.0 & Decontamination \& Decommissioning & $\$ 77.6$ & 1.09 & 1.05 & 1.00 & 1.00 & 0.97 \\
\hline 7.0 & Planning life cycle costs (20 years operation) & $\$ 3,109.7$ & 1.07 & 0.97 & 1.01 & 1.13 & 0.99 \\
\hline
\end{tabular}

Notes:

1. INTS System 5 costs are taken as the normalizing cost basis.

2. Actual Costs are in millions of dollars. 
Table 4-9. TLCC Comparison (without disposal) by Subsystem for the Five ENTS systems (in millions of dollars).

\begin{tabular}{|c|c|c|c|c|c|}
\hline SUBSYSTEM & $\begin{array}{c}\text { ENTS } 1 \\
(\$ \mathrm{M}) \\
\end{array}$ & $\begin{array}{c}\text { ENTS } 2 \\
(\$ \mathrm{M})\end{array}$ & $\begin{array}{c}\text { ENTS 3 } \\
(\$ M) \\
\end{array}$ & $\begin{array}{c}\text { ENTS } 4 \\
\text { (\$M) }\end{array}$ & $\begin{array}{c}\text { ENTS } 5 \\
(\$ M) \\
\end{array}$ \\
\hline Administration Building Subsystem & 169.9 & 169.9 & 169.9 & 169.9 & 169.9 \\
\hline Receiving \& Inspection Subsystem & 940.5 & 940.5 & 940.5 & 940.5 & 940.5 \\
\hline Aqueous Waste Treatment Subsystem & 149.7 & 149.7 & 149.7 & 149.7 & 149.7 \\
\hline Organic Destruction Subsystem & 177.0 & 177.0 & 177.0 & 177.0 & 358.9 \\
\hline Residue \& Sludge Treatment Subsystem & 126.7 & $\begin{array}{l}\text { Treated by other } \\
\text { subsystems }\end{array}$ & 126.7 & 126.7 & 126.7 \\
\hline Soft Debris Treatment Subsystem & 219.3 & 219.3 & 219.3 & 219.3 & \\
\hline Complex Debris Treatment Subsystem & $\begin{array}{l}\text { Treated by other } \\
\text { subsystems }\end{array}$ & $\begin{array}{l}\text { Treated by other } \\
\text { subsystems }\end{array}$ & $\begin{array}{l}\text { Treated by other } \\
\text { subsystems }\end{array}$ & $\begin{array}{l}\text { Treated by other } \\
\text { subsystems }\end{array}$ & $\begin{array}{l}\begin{array}{l}\text { Treated by other } \\
\text { subsystems }\end{array} \\
\text { unsy }\end{array}$ \\
\hline Open Debris Treatment Subsystem & 98.5 & 98.5 & 98.5 & 98.5 & 98.5 \\
\hline Bulk Soil Treatment Subsystem & 54.8 & 54.8 & 54.8 & 54.8 & 54.8 \\
\hline Metal Decontamination Subsystem & 64.2 & 64.2 & 64.2 & 64.2 & 64.2 \\
\hline Special Waste & 45.9 & 45.9 & 45.9 & 45.9 & 45.9 \\
\hline Mercury Amalgamation Subsystem & 58.0 & 58.0 & 34.7 & 34.7 & 34.7 \\
\hline Lead Recovery Subsystem & 89.0 & 89.0 & 41.1 & 41.1 & 41.1 \\
\hline Air Pollution Control Subsystem & 70.4 & 70.4 & 70.4 & 70.4 & 76.0 \\
\hline Polymer Stabilization Subsystem & 289.2 & 163.5 & 443.8 & 479.8 & 289.2 \\
\hline Vitrification & 252.4 & 303.1 & & & \\
\hline Grout Stabilization Subsystem & & & & & 120.1 \\
\hline Certification \& Shipping Subsystem & 346.4 & 223.5 & 423.9 & 757.8 & 423.9 \\
\hline Support Subsystem & 83.8 & 83.8 & 83.8 & 83.8 & 83.8 \\
\hline Metal Melting Subsystem & 90.4 & 90.4 & & 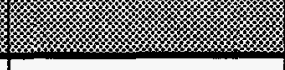 & \\
\hline TOTAL & $3,326.1$ & $3,001.5$ & 3,144.2 & $3,514.1$ & $3,078.1$ \\
\hline
\end{tabular}


Table 4-10. Operating FTE Estimates for ENTS Systems.

\begin{tabular}{|c|c|c|c|c|c|c|}
\hline SUBSYSTEM & ENTS 1 & ENTS 2 & ENTS 3 & ENTS 4 & ENTS 5 & INTS 5 \\
\hline Administration Building Subsystem & 32 & 32 & 32 & 32 & 32 & 32 \\
\hline Receiving \& Inspection Subsystem & 170 & 170 & 170 & 170 & 170 & 170 \\
\hline Aqueous Waste Treatment Subsystem & 5 & 5 & 5 & 5 & 5 & 5 \\
\hline Organic Destruction Subsystem & 12 & 12 & 12 & 12 & 15 & 12 \\
\hline Residue \& Sludge Treatment Subsystem & 8 & 0 & 8 & 8 & 8 & 8 \\
\hline Soft Debris Treatment Subsystem & 14 & 14 & 14 & 14 & 0 & 14 \\
\hline Complex Debris Treatment Subsystem & \begin{tabular}{|l|l} 
Treated by other \\
subbyystems
\end{tabular} & \begin{tabular}{|l|} 
Treated by other \\
subsyytems
\end{tabular} & \begin{tabular}{|l|l} 
Treated by other \\
subsystems
\end{tabular} & \begin{tabular}{|l|l|} 
Treated by other \\
subbyystems
\end{tabular} & \begin{tabular}{l|l} 
Treated by other \\
subsystems
\end{tabular} & $\begin{array}{l}\text { Treated by other } \\
\text { subsystems }\end{array}$ \\
\hline Open Debris Treatment Subsystem & 8 & 8 & 8 & 8 & 8 & 8 \\
\hline Bulk Soil Treatment Subsystem & 2 & 2 & 2 & 2 & 2 & 2 \\
\hline Metal Decontamination Subsystem & 4 & 4 & 4 & 4 & 4 & 4 \\
\hline Special Waste & 3 & 3 & 3 & 3 & 3 & 3. \\
\hline Mercury Amalgamation Subsystem & 4 & 4 & 1 & 1 & 1 & 1 \\
\hline Lead Recovery Subsystem & 8 & 8 & 3 & 3 & 3 & 3 \\
\hline Air Pollution Control Subsystem & 4 & 4 & 4 & 4 & 4 & 4 \\
\hline Polymer Stabilization Subsystem & 38 & 8 & 56 & 45 & 38 & 37.5 \\
\hline Vitrification & 16 & 18 & & & & \\
\hline Grout Stabilization Subsystem & & & & & 8 & 8 \\
\hline Certification \& Shipping Subsystem & 67 & 37 & 88 & 175 & 88 & 88 \\
\hline Support Subsystem & 5 & 5 & 5 & 5 & 5 & 5 \\
\hline Metal Melting Subsystem & 5 & 5 & & & & 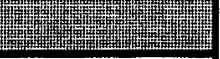 \\
\hline TOTAL & 405 & 339 & 415 & 491 & 394 & 405 \\
\hline
\end{tabular}


Table 4-11. Reaction Rates for CWO.

\begin{tabular}{|c|c|c|}
\hline COMPOUND & $\begin{array}{c}\text { DELPHI REACTION RATE AT } \\
200^{\circ} \mathrm{C} \\
\mathrm{kg} / \mathrm{hr} \\
\end{array}$ & $\begin{array}{l}\text { EQUIVALENT INTS } \\
\text { WASTE CATEGORY }\end{array}$ \\
\hline \multicolumn{3}{|l|}{ VAPORS: } \\
\hline Acetone & 0.39 & Nonhalogenated Organic Liquids \\
\hline MEK & 0.487 & Nonhalogenated Organic Liquids \\
\hline Hexane & 0.585 & Nonhalogenated Organic Liquids \\
\hline TCE & 0.885 & Halogenated Organic Liquids \\
\hline Chloroform & 0.8025 & Halogenated Organic Liquids \\
\hline Methylene chloride & 0.57 & Halogenated Organic Liquids \\
\hline Carbon tetrachloride & 1.035 & Halogenated Organic Liquids \\
\hline $1,1,1 \mathrm{TCA}$ & 0.9 & Halogenated Organic Liquids \\
\hline Methanol & 0.3 & Nonhalogenated Organic Liquids \\
\hline & & \\
\hline \multicolumn{3}{|l|}{ LIQUIDS: } \\
\hline oil & 0.041 & oil \\
\hline $\begin{array}{c}\text { Weighted Average Reaction Rate } \\
\text { for Liquids and Vapors }\end{array}$ & 0.191 & \\
\hline Reaction Rate at 10 times actual & 1.91 & \\
\hline 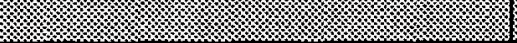 & 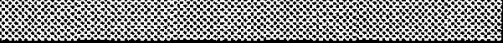 & \\
\hline \multicolumn{3}{|l|}{ SOLIDS: } \\
\hline paper & 0.12 & paper, cardboard \\
\hline cotton & 0.18 & cloth \\
\hline wood & 0.16 & wood \\
\hline latex rubber & 0.033 & rubber \\
\hline butyl rubber & 0.0066 & rubber \\
\hline PVC & 0.14 & PVC \\
\hline Polyethylene & 0.0049 & $\mathrm{PE} / \mathrm{PP}$ \\
\hline activated carbon & 0.0009 & graphite \\
\hline ion exchange resin & 0.0065 & all other nonplastic \\
\hline × & 症: & (1) \\
\hline Weighted Average Rate for Solids & 0.102 & 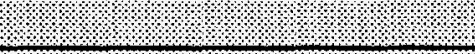 \\
\hline Reaction Rate at 10 times actual & 1.02 & (: \\
\hline
\end{tabular}

Notes:

1. Rates based on Delphi Research, Inc data (Dhooge, 1996). Delphi representatives stated that a 7.5 times increase was appropriate to adjust the test data from 150 to $200^{\circ} \mathrm{C}$. This adjustment is included in this table.

2. Weighted average rates were calculated by

a) calculating the percent distribution of each of the identified waste types in INTS organic liquids and soft debris

b) multiplying the percent distribution by the reaction rate for that waste

c) summing the product of the percent distribution by the reaction rate to get the total reaction rate.

3. To convert from $\mathrm{kg} / \mathrm{h}$-hr to $\mathrm{lb} / \mathrm{gal}-\mathrm{hr}$, multiply by 8.38 . 


\section{SYSTEM COMPARISON}

This section summarizes the results for representative thermal systems evaluated in the ITTS study and nonthermal systems evaluated in the INTS study, and compares these results with the results for the enhanced nonthermal systems evaluated in this study.

The thermal systems compared include the following:

\section{System A-1}

- rotary kiln with air for combustion for organic destruction

- vitrification of ash

- polymer stabilization of secondary wastes

- decontamination of surface contaminated metals

- melting of metals with entrained contamination, and

- retorting of mercury contaminated waste

\section{System C-1}

- plasma furnace with air for combustion for organic destruction

- polymer stabilization of secondary wastes

- decontamination of surface contaminated metals

- melting of metals with entrained contamination, and

- retorting of mercury contaminated waste

\section{System H-1}

- steam reforming of combustible waste for organic destruction

- vitrification of ash and inorganic waste

- polymer stabilization of secondary wastes

- decontamination of surface contaminated metals

- melting of metals with entrained contamination, and

- retorting of mercury contaminated waste

The nonthermal systems compared include the following:

\section{System NT-1}

- mediated electrochemical oxidation for organic destruction

- thermal desorption of soil and process residue

- decontamination of surface contaminated metals

- polymer stabilization of treated sludge and secondary waste

- grout stabilization of treated soil and untreated debris

- amalgamation of mercury contaminated waste 


\section{System NT-2}

- catalytic wet oxidation for organic destruction

- thermal desorption of soil, process residue and debris

- decontamination of surface contaminated metals

- polymer stabilization of treated sludge and secondary waste

- grout stabilization of treated soil and treated debris

- amalgamation of mercury contaminated waste

\section{System NT-3}

- mediated electrochemical oxidation for organic destruction

- washing of soil, process residue and debris

- decontamination of surface contaminated metals

- polymer stabilization of treated sludge and secondary waste

- grout stabilization of treated soil and treated debris

- amalgamation of mercury contaminated waste

\section{System NT-4}

- acid digestion for organic destruction of liquids and soft (combustible debris)

- thermal desorption of process residue

- washing of soil and debris

- decontamination of surface contaminated metals

- stabilization of treated waste in phosphate bonded ceramic

- stabilization of secondary waste in polymer or phosphate bonded ceramic

- amalgamation of mercury contaminated waste

\section{System NT-5}

- catalytic wet oxidation for organic destruction of liquids and soft (combustible debris)

- thermal desorption of process residue

- washing of soil and debris

- decontamination of surface contaminated metals

- polymer stabilization of treated sludge and secondary wastes

- primary stabilization of treated soil and treated debris in grout

- amalgamation of mercury contaminated waste

\subsection{Feed Rates}

The waste feed rates to the various subsystems for the systems being compared are shown in Table 5-1. The administration and receiving and preparation subsystems must handle the same amount of material (2927 $\mathrm{lbs} / \mathrm{hr}$ ) for all systems.

Aqueous waste treatment for INTS or ENTS systems treats approximately the same amount of wastewater, whereas ITTS systems treat 3 to 10 times more wastewater than INTS or ENTS systems. Most of the wastewater treated in thermal systems is scrubber liquor from the air pollution control subsystem. This 
wastewater stream has few or no organic contaminants and is therefore evaporated rather than being passed through a complex treatment process. Thus, as will be seen later, the cost of the aqueous waste treatment subsystem for all systems is approximately the same.

The organic destruction subsystem for thermal systems treats most of the incoming waste (organic wastes, soil, sludge and all debris), whereas in nonthermal systems only organic material (organic liquid and organic sludge) is treated in the organic destruction process. In nonthermal systems soft (or combustible) debris is treated by stabilization (System NT-1), thermal desorption (System NT-2) or washing (System NT-3), followed by stabilization. In Systems NT-4 and NT-5 soft debris is destroyed using the same chemical reagents used to destroy organic liquids and sludges, but different reaction vessels are used. In the nonthermal systems only acid digestion and catalyzed wet oxidation (CWO) can treat all organic waste including soft debris. Noncombustible waste requires processes to separate organic contaminants from the inorganic matrix. The separated organics are then sent to the destruction process.

Metal debris with entrained contamination (complex debris) is melted in a metal melter in most thermal systems. However, in System C-1 the plasma furnace can accept this waste stream and separate the metal from the slag to form ingots. In nonthermal systems the EPA Debris Rule is invoked and metal items that fall into this category are macroencapsulated in grout or polymer. In hybrid systems ENTS-1 and ENTS-2 metal with entrained contamination is also melted into ingots.

The enhanced nonthermal systems are similar to System NT-5 in which CWO is used to treat organic waste and combustible debris. In all systems, except ENTS-5, organic waste and soft debris are treated in separate reaction vessels. In system ENTS-5 the assumed reaction rate is fast enough that a single reaction vessel of reasonable size (1,000 gallons) can be used to treat all organic material, including soft debris.

All enhanced nonthermal systems except System ENTS-2 require separation of organics from noncombustible waste in the same manner as the nonthermal systems. In ENTS-2 inorganic sludge ( $\sim 34$ percent of the incoming waste) is sent directly to a vitrifier which destroys the organic contaminants and vitrifies the sludge. However, in this case organics are still separated from soils and inorganic debris by being washed prior to vitrification of the inorganic waste streams. System H-1 is similar to ENTS-2 in that the steam reformer can treat only combustible material while all noncombustible material (except metals) is sent directly to the vitrifier.

Thermal systems (except System H-1 which requires a steam reformer and a vitrifier) can treat most of the waste in a single process. Nonthermal systems require separate processes for organic waste and soft debris, and require additional processes for inorganic waste streams to separate the organic contaminants from the inorganic matrix.

Enhanced nonthermal systems require separate processes for organic waste and soft debris unless the feed system, reactor size, and process controls can be designed such that all combustible waste can be treated in the same reaction vessel. Enhanced nonthermal systems also require separation of organics from inorganic waste matrices unless a vitrifier can be demonstrated to reliably treat organically contaminated soils, sludge and debris.

Because most thermal systems use excess air to oxidize combustible material, they have significantly more offgas requiring treatment in the air pollution control subsystem than do nonthermal or enhanced nonthermal systems. The offgas entering the APC subsystem for System H-1 is a combustible gas from the steam reformer. This gas is oxidized with excess air in a thermal oxidizer so that the total gaseous emissions from System H-1 are significantly higher than the input shown for the APC system. 


\subsection{Effluent}

Effluent volumes for the thermal, nonthermal and enhanced nonthermal systems is shown in Table 5-2. The offgas emissions are shown in Figure 5-1. The offgas from nonthermal and enhanced nonthermal systems is about one-tenth to one-third the offgas from thermal systems. This is due to the need for excess air for combustion in the thermal systems where the major component of the offgas is nitrogen as shown in Table 5-3. The undefined portion of the offgas from thermal systems is the offgas from ancillary operations such as metal decontamination and should have essentially the same composition as the other sources.

In condensed phase (or liquid phase) organic destruction used in nonthermal systems, organics are converted to carbon dioxide and water without the additional air or oxygen for combustion. Thus, the primary component of the offgas from these systems is carbon dioxide. More $\mathrm{CO}_{2}$ is produced in Systems NT-4 and NT-5 and the enhanced nonthermal systems, compared to NT-1, NT-2, and NT-3. This is because soft debris, in addition to organic liquid and sludge, is oxidized by acid digestion or CWO in these systems. The other nonthermal systems only oxidize organic liquid and sludge.

The enhanced nonthermal systems that use vitrification have slightly more offgas than do the other enhanced nonthermal or nonthermal systems. This is because excess air is used in the vitrifier's secondary combustion chamber to oxidize any organics volatilized in the vitrifier. System ENTS-2 has the most offgas because of the organics in the untreated sludge sent to the vitrifier. However, this offgas is still an order-ofmagnitude less than the offgas from System A-1 and one-third the offgas from System C-1.

The wastewater discharged from the four enhanced nonthermal systems that use polymer or vitrification for stabilization is greater than NT-5 or ENTS-5 which use grout. This is because some wastewater is used in the grouting process and is tied up in the grout rather than discharged.

Hybrid systems ENTS-1 and ENTS-2 have about the same amount of clean metal going to recycling as do the thermal systems, whereas nonthermal systems have less metal to be recycled. This is because thermal and hybrid systems melt metals with entrained contamination and produce ingots that are assumed to be recycled within the DOE complex. Nonthermal systems macroencapsulate these metal debris items and dispose of them.

Systems that produce a vitrified final waste form have significantly less mass sent to disposal than systems that use a nonthermal stabilization material such as grout or polymer. Production of a glass/ceramic waste form in a vitrifier requires addition of one part glass former for every two parts waste. Polymer requires one part polymer to one part waste, while grout requires two parts grout material to one part waste. Thus, nonthermal stabilization requires significantly more stabilizing material to be added to the waste than does vitrification. Systems NT-4 and NT-5 have less final waste form mass than the other nonthermal systems because soft debris is destroyed instead of being stabilized.

System ENTS-1 has a greater amount of solids sent to disposal than thermal systems because in ENTS-1 treated inorganic sludge (or process residue) is stabilized in polymer. However, replacing grout in System NT-5 with vitrification produces less final waste form volume for disposal. System ENTS-2 has nearly the same amount of stabilized waste as do the thermal systems because most of the solid waste is vitrified in both cases. Replacing grout with polymer (ENTS-3) also slightly decreases the mass and volume of waste sent to disposal. System ENTS-4 has the highest final waste form volume because the sintering (or Claytec) process requires a one-to-one mixture of clay and waste followed by the addition of polymer at 35 percent of the waste plus clay on a mass basis. 


\subsection{Waste Sent to Disposal}

The mass of the solid waste sent to disposal as a stabilized final waste form is shown in Table 5-4 and Table 5-5. Figure 5-2 presents the volume of solid waste sent to disposal. Replacing grout in NT-5 with vitrification as in ENTS-1 produces a decrease in the final waste form volume from $36.1 \mathrm{cu}$. $\mathrm{ft}$ to $24.7 \mathrm{cu}$. $\mathrm{ft}$ and thus reduces the disposal cost from $\$ 707$ million to $\$ 484$ million. System ENTS-2 extends the trend of volume reduction by the vitrification of untreated inorganic sludge. For System ENTS-2, the waste form volume is reduced to $14.6 \mathrm{cu}$. $\mathrm{ft}$ and the disposal cost to $\$ 286$ million. Stabilizing the waste in polymer only (ENTS-3) decreases the final waste form volume slightly from $36.1 \mathrm{cu}$. ft in NT-5 to $35.1 \mathrm{cu}$. ft .

System ENTS- 4 has the most mass and volume sent to disposal because of the combined stabilization process. If the pellets produced by the Claytec process can be shown to pass the TCLP tests then macroencapsulation may not be required. In this case, the volume sent to disposal would be reduced from $46.5 \mathrm{cu}$. $\mathrm{ft}$ to $35.1 \mathrm{cu}$. $\mathrm{ft}$, the same as ENTS-3 that uses polymer stabilization. This is because both the polymer and Claytec processes use a 1:1 ratio of stabilizing agent to waste and the same assumed density of the stabilized waste $(94 \mathrm{lbs} / \mathrm{cu}$. ft) for both cases. If the density of the pellets is greater than the density of the polymer stabilized waste, then the volume of disposed waste may be less, although the void fraction of containerized pellets would need to be considered in calculating the total volume.

Vitrified waste has a lower total mass than waste stabilized by nonthermal means. This is because fewer additives are required to produce the final waste form. Vitrified waste has a higher density than grout or polymer so the volume of waste is significantly less (by approximately a factor of three). Thus, hybrid systems, such as ENTS-2 in which all primary inorganic waste is vitrified, minimize both the offgas and the volume of waste sent to disposal. However, not all waste can be vitrified because there are some secondary waste salts that will either cause problems with the vitrifier or will volatilize and therefore cannot be contained in the glass. These secondary wastes will require a nonthermal stabilization such as polymer.

The volume reduction factor (volume in/volume out) is shown in Table 5-6 where the density of incoming waste is assumed to be $64 \mathrm{lbs} / \mathrm{ft}^{3}$. The percent of the volume of waste entering the facility that leaves the facility as a final waste form (output/input expressed as a percent) is presented in Table 5-6 and Figure 5-3. For systems that use nonthermal stabilization the volume of waste is reduced by 5 to 10 percent for those systems that do not destroy soft debris, and by 20 percent for those systems that do destroy soft debris (Systems NT-4 and NT-5).

The waste volume is reduced by 70 percent for thermal systems. The volume reduction for System ENTS-3 which uses polymer is also 20 percent, about the same as NT-5 which uses grout and polymer. There is a volume increase for System ENTS-4 which uses an enhanced hybrid final waste form. Volume reduction for System ENTS-2 is about the same as for thermal systems ( $\sim 70$ percent).

\subsection{Resources Used}

Table 5-7 illustrates the energy use for the systems being compared. The highest costs for electrical energy are associated with those systems that use electrical energy for organic destruction (plasma furnace), or to regenerate the oxidizer in the organic destruction process (mediated electrochemical oxidation in Systems NT-1 and NT-3).

Systems that vitrify treated waste use an intermediate amount of electrical energy. Systems that have the highest natural gas cost are those that use combustion for organic destruction (e.g., System A-1). In all cases, energy costs are less than one percent of the treatment costs (i.e., TLCC without disposal). 
The reagent use by these systems is shown in Table 5-8. The enhanced nonthermal systems use approximately the same quantity of reagents as System NT-5 since the enhanced nonthermal systems use the same organic destruction process.

The major reagent used in all systems is sodium hydroxide. This is used for acid neutralization in nonthermal systems, and for scrubber water and acid gas neutralization in thermal systems.

Truck traffic to transport reagents to the treatment facility is minimal at 14 to 27 trucks per year as shown in Table 5-9. The major source of truck traffic is incoming waste, which is the same for all systems, and stabilizing agents. Systems that use vitrification have the least number of incoming trucks (340 to 350 trucks/year) and the least number of outgoing trucks taking vitrified waste to disposal and metals to storage (230 to 250 trucks/year). Systems that use nonthermal stabilization have more incoming traffic (450 to 520 trucks/year) to deliver stabilizing agent, and more outgoing traffic to dispose of the greater volume of stabilized waste.

The FTE requirements for these systems is shown in Table 5-10 for each subsystem. The major requirements are in the front-end handling subsystem to receive, characterize, sort and size reduce the incoming waste.

Nonthermal systems (including the enhanced nonthermal systems) require much more sorting than thermal systems so that the FTEs required for front-end handling are significantly greater. A large number of personnel are required in the certification and shipping subsystem for nonthermal systems because the volume of waste passing through this subsystem is greater for nonthermal than for thermal systems. The personnel required in certification and shipping for the enhanced nonthermal systems is also a function of the volume of stabilized waste passing through the subsystem. The personnel required for System ENTS-2 is approximately equal to the personnel required for thermal systems.

The personnel required for organic destruction in Systems NT-5 and ENTS-5 include personnel to treat organic liquid, sludge and soft debris. Because of the increased reaction rate in ENTS-5, these waste streams can be treated in one reaction vessel and in one shift, so fewer operating personnel are required for ENTS-5.

\subsection{Life Cycle Cost Comparison}

Life cycle costs are shown by work breakdown element in Table 5-11 and Figure 5-4. In all cases operating and maintenance (O\&M) costs are 50 to 60 percent and capital costs are 15 to 25 percent of the TLCC. Disposal costs depend on the volume of the final waste form and range from about 10 percent of the TLCC for systems that use vitrification to 20 percent of the TLCC for systems that use nonthermal stabilization technologies.

Treatment costs (i.e., life cycle cost without disposal) for nonthermal systems and three of the enhanced nonthermal systems (ENTS-1, ENTS-3 and ENTS-4) are about \$1 billion more than for thermal systems. Treatment costs for System ENTS-1 is about $\$ 1.2$ billion and System ENTS-4 is about \$1.4 billion more than the thermal systems. These two ENTS systems require two stabilization processes. Their costs reflect the higher costs associated with vitrification and the Claytec processes, respectively.

The major driver for these higher treatment costs is the higher O\&M costs associated with nonthermal and enhanced nonthermal systems due to more sorting and segregating of waste, higher volumes of waste passing through the certification and shipping subsystem, and more unit operations required to treat the waste.

Disposal costs for systems that use vitrification are a factor of 2.5 to 3.5 less than for systems that use nonthermal stabilization. Disposal costs for System ENTS-2 are about the same as for thermal systems and 
considerably less than nonthermal systems. Disposal costs for System ENTS-1 are less than those for nonthermal systems, but higher than those for thermal systems since a significant portion of the waste (inorganic sludge at 34 percent of the incoming waste) is stabilized in polymer.

Life cycle costs broken down by subsystem are shown in Table 5-12. The major cost driver for all systems is the front-end handling subsystem at 20 to 30 percent of the TLCC. Stabilization costs are 12 to 15 percent of the TLCC for all systems, and metal recovery costs are higher for systems using metal melting. It costs $\$ 90$ million (Table 4-9) over 20 years to melt $149 \mathrm{lbs} / \mathrm{hr}$ of ferrous metal with entrained contamination and to produce ingots for possible recycling within the DOE complex. However, stabilization of the same internally contaminated metal in polymer produces 2.17 cubic feet per hour of stabilized waste that costs $\$ 43$ million for disposal.

Primary treatment costs are about the same for all nonthermal and enhanced nonthermal systems that destroy soft debris, but these costs are about three times the primary treatment costs of thermal systems due to the multiple subsystems required.

\subsection{Conclusions}

Several conclusions can be drawn from this comparison of systems. There is no economic incentive to use nonthermal or enhanced nonthermal systems in place of thermal systems in a centralized treatment facility. However, reduced offgas and low final waste form volumes may make hybrid systems attractive.

System ENTS-2 has one-third the offgas from the plasma furnace (System C-1) and one-tenth the offgas from the rotary kiln (System A-1), with approximately the same final waste form volume at a cost of $\$ 1$ billion more than these two thermal systems.

The costs for System ENTS-2 may be reduced by eliminating the washing process ( $\$ 153$ million) and treating all inorganic waste in the vitrifier as in System H-1. This should also reduce the front-end handling costs somewhat, as less sorting will be required. However, a vitrification subsystem must be demonstrated to reliably and efficiently destroy the organic contaminants in these inorganic matrices.

The more waste that can be vitrified, the lower the final waste form volume, and, thus, the lower the disposal cost.

The use of polymer to stabilize all waste (ENTS-3) reduces the final waste form volume slightly from that produced by System NT-5 which uses grout and polymer. Although there is little cost incentive to use all polymer, a waste form with better performance characteristics than grout may be produced.

Using hybrid waste forms (pellets macroencapsulated in polymer as in ENTS-4) may have improved performance but at a cost of $\$ 550$ million more than System ENTS-3 which uses only polymer for stabilization. If the compressed and sintered pellets can pass TCLP without macroencapsulation, then the stabilization and disposal costs should decrease and be closer to those of System ENTS-3.

There is no cost incentive to perform $R \& D$ to increase the reaction rate of nonthermal organic destruction processes. Increasing the reaction rate will allow treatment of all organic wastes in a single subsystem and in a shorter period of time. This decreases the capital and operating cost for treatment. However, this savings is offset by the increase in maintenance costs associated with equipment and personnel required to handle the increased throughput, and the cost of equipment required to produce the increased reaction rate. An advantage of an increased reaction rate may be an increase in the destruction efficiency if the residence time were to be kept the same as the original residence time with the lower reaction rate (i.e., the throughput remains the same). 
Nonthermal systems may be made more competitive with thermal systems by enhancing them through the use of vitrification for all inorganic wastes (except metals and secondary waste salts) and by eliminating the organic separation processes (thermal desorption and washing). This should reduce the capital costs and O\&M costs required to operate and maintain this equipment. Sorting requirements, and therefore the frontend handling costs, should decrease. In addition, the final waste form volume and therefore certification and shipping costs and disposal costs should also decrease. Thus, the most competitive enhanced nonthermal system includes only one nonthermal process (destruction of organic waste, including combustible debris). Detailed evaluation of a hybrid system such as its comparison with selected thermal systems would quantify how competitive a hybrid system can become. 
Table 5-1 Subsystem Waste Feed Rates (lbs/hr)

\begin{tabular}{|c|c|c|c|c|c|c|c|c|}
\hline Subsystems ${ }^{a}$ & $\begin{array}{c}\text { Rotary } \\
\text { Kiln/Air } \\
(A-1)\end{array}$ & $\begin{array}{l}\text { Plasma } \\
\text { Furnace } \\
(\mathrm{C}-1)\end{array}$ & $\begin{array}{c}\text { Steam } \\
\text { Reforming } \\
(\mathrm{H}-1)\end{array}$ & $\begin{array}{l}\text { Grout } \\
\text { Debris } \\
\text { (NT-1) }\end{array}$ & $\begin{array}{c}\text { Thermal } \\
\text { Desorption } \\
(\mathrm{NT}-2)\end{array}$ & $\begin{array}{c}\text { Wash } \\
(\mathrm{NT}-3)\end{array}$ & $\begin{array}{c}\text { Acid } \\
\text { Digestion } \\
(\mathrm{NT}-4) \\
\end{array}$ & $\begin{array}{c}\text { Catalytic Wet } \\
\text { Oxidation } \\
\text { (NT-5) }\end{array}$ \\
\hline Administration & 2927 & 2927 & 2927 & 2927 & 2927 & 2927 & 2927 & 2927 \\
\hline Receiving and Preparation & 2927 & 2927 & 2927 & 2927 & 2927 & 2927 & 2927 & 2927 \\
\hline Aqueous Waste Treatment & 13658 & 6568 & 3266 & 1193 & 1258 & 1632 & 1983 & 1956 \\
\hline Organic Destruction & 2000 & 2237 & 660 & 237 & 268 & 326 & 256 & 256 \\
\hline Process Residue & $b$ & $b$ & ${ }^{\circ}$ & 1020 & 1020 & 1020 & 1020 & 1020 \\
\hline Bulk Soil Treatment & $b$ & 6 & ${ }^{\circ}$ & 329 & 329 & 329 & 329 & 329 \\
\hline Soft Debris Treatment & b & b & $\mathrm{b}$ & d & 435 & 435 & 435 & 435 \\
\hline Open Debris Treatment & b & $b$ & c & d & 80 & 80 & 80 & 80 \\
\hline Complex Debris Treatment & $b$ & $\mathrm{~b}$ & $\circ$ & d & 177 & 177 & $\cdot$ & $e$ \\
\hline Metal Decontamination & 468 & 468 & 468 & 462 & 462 & 462 & 462 & 462 \\
\hline Mercury Amalgamation & 50 & 50 & 50 & 2.6 & 2.6 & 2.5 & 2.6 & 2.6 \\
\hline Lead Recovery & 21 & 21 & 21 & 26 & 26 & 26 & 26 & 26 \\
\hline Metal Melting & 154 & 5 & 154 & Not Used & Not Used & Not Used & Not Used & Not Used \\
\hline Polymer Stabilization & 123 & 123 & 123 & 1067 & 1110 & 1246 & 6.6 & 1126 \\
\hline Phosphate Bonded Ceramic & Not Used & Not Used & Not Used & Not Used & Not Used & Not Used & 1592 & Not Used \\
\hline Claytec Process & Not Used & Not Used & Not Used & Not Used & Not Used & Not Used & Not Used & Not Used \\
\hline Vitrification & 1495 & Not Used & 1490 & Not Used & Not Used & Not Used & Not Used & Not Used \\
\hline Grout Stabilization & 0.3 & 0.3 & 0.3 & 1082 & 1228 & 958 & 265 & 650 \\
\hline Air Pollution Control & 21420 & 7339 & 957 & 695 & 823 & 827 & 1396 & 1433 \\
\hline Special Waste & 153 & 153 & 153 & 155 & 155 & 155 & 155 & 155 \\
\hline Certification \& Shipping & 2551 & 2519 & 2549 & 5126 & 5067 & 4919 & 4115 & 4270 \\
\hline
\end{tabular}

Notes:

"Input to all systems includes only primary and secondary waste streams

These waste streams are sent to organic destruction

'These waste streams are sent to vitrification.

These waste streams are sent to grout stabilization

'Complex debris is treated by grout stabilization

Soft debris is treated by organic destruction

B Complex debris is treated by polymer stabilization.

${ }^{h}$ Claytec and polymer macroencapsulation are used in ENTS-4. 
Table 5-1 (continued) Subsystem Waste Feed Rates (lbs/hr)

\begin{tabular}{|c|c|c|c|c|c|}
\hline Subsystems & $\begin{array}{l}\text { Replace Grout with } \\
\text { Vitrification } \\
\text { (ENTS-1) } \\
\end{array}$ & $\begin{array}{c}\text { Vitrify Untreated } \\
\text { Sludge } \\
\text { (ENTS-2) } \\
\end{array}$ & $\begin{array}{c}\text { Polymer } \\
\text { Stabilization } \\
\text { (ENTS-3) } \\
\end{array}$ & $\begin{array}{c}\text { Pelletization } \\
\text { with Polymer } \\
\text { (ENTS-4) } \\
\end{array}$ & $\begin{array}{c}\text { Increased } \\
\text { Reaction Rate } \\
\text { (ENTS-5) } \\
\end{array}$ \\
\hline Administration & 2927 & 2927 & 2927 & 2927 & 2927 \\
\hline Receiving and Preparation & 2927 & 2927 & 2927 & 2927 & 2927 \\
\hline Aqueous Waste Treatment & 1945 & 1653 & 1914 & 1914 & 1956 \\
\hline Organic Destruction & 256 & 233 & 256 & 256 & 691 \\
\hline Process Residue & 1007 & ${ }^{\circ}$ & 1007 & 1007 & 1020 \\
\hline Bulk Soil Treatment & 329 & 329 & 329 & 329 & 329 \\
\hline Soft Debris Treatment & 435 & 435 & 435 & 435 & $e^{\circ}$ \\
\hline Open Debris Treatment & 80 & 80 & 80 & 80 & 80 \\
\hline Complex Debris Treatment & 177 & 177 & $\mathbf{B}$ & $\mathrm{g}$ & $\mathrm{e}^{\mathrm{e}}$ \\
\hline Metal Decontamination & 462 & 462 & 462 & 462 & 462 \\
\hline Mercury Amalgamation & 3.9 & 3.8 & 2.6 & 2.6 & 2.6 \\
\hline Lead Recovery & 21 & 21 & 26 & 26 & 26 \\
\hline Metal Melting & 154 & 154 & Not Used & Not Used & Not Used \\
\hline Polymer Stabilization & 1128 & 176 & 1772 & $h$ & 1126 \\
\hline Phosphate Bonded Ceramic & Not Used & Not Used & Not Used & Not Used & Not Used \\
\hline Claytec Process & Not Used & Not Used & Not Used & 1772 & Not Used \\
\hline Vitrification & 495 & 1502 & Not Used & Not Used & Not Used \\
\hline Grout Stabilization & Not Used & Not Used & Not Used & Not Used & 650 \\
\hline Air Pollution Control & 1507 & 1731 & 1433 & 1433 & 1433 \\
\hline Special Waste & 155 & 155 & 155 & 155 & 155 \\
\hline Certification \& Shipping & 3227 & 2701 & 3687 & 4762 & 4270 \\
\hline
\end{tabular}

Notes:

'Input to all systems includes only primary and secondary waste streams

${ }^{b}$ These waste streams are sent to organic destruction

'These waste streams are sent to vitrification.

These waste streams are sent to grout stabilization

"Complex debris is teat by grout stabilization

Complex debris is trealed by grout stabilization

Complex debris is treated by polymer stabilization.

"Claytec and polymer macroencapsulation are used in ENTS-4. 
Table 5-2 System Effluents (lbs/hr)

\begin{tabular}{|c|c|c|c|c|c|c|c|c|}
\hline Effluent (lbs/hr) & $\begin{array}{c}\text { Rotary Kiln } \\
\text { /Air } \\
\text { (A-1) }\end{array}$ & $\begin{array}{l}\text { Plasma } \\
\text { Furnace } \\
(\mathrm{C}-1)\end{array}$ & $\begin{array}{c}\text { Steam } \\
\text { Reforming } \\
(\mathrm{H}-1)\end{array}$ & $\begin{array}{l}\text { Grout Debris } \\
\text { (NT-1) }\end{array}$ & $\begin{array}{l}\text { Thermal } \\
\text { Desorption } \\
\text { (NT-2) }\end{array}$ & $\begin{array}{l}\text { Wash } \\
\text { (NT-3) }\end{array}$ & $\begin{array}{c}\text { Acid } \\
\text { Digestion } \\
\text { (NT-4) }\end{array}$ & $\begin{array}{l}\text { Catalytic Wet } \\
\text { Oxidation } \\
\text { (NT-5) }\end{array}$ \\
\hline Offgas & 20697 & 6895 & 11648 & 696 & 823 & 827 & 101441 & 1433 \\
\hline Wastewater & 399 & 463 & -487 & 59 & 135 & 176 & 780 & 564 \\
\hline Clean Metal to Recycle & 622 & 658 & 622 & 451 & 451 & 451 & 451 & 451 \\
\hline Clean Lead & 24.5 & 24.5 & 24.5 & 21 & 21 & 21 & 21 & 21 \\
\hline Clean Metal & 597 & 633 & 597 & 430 & 430 & 430 & 430 & 430 \\
\hline Solids to Disposal & 1929 & 1861 & 1927 & 4675 & 4616 & 4468 & 3664 & 3819 \\
\hline Mercury Amalgam & 10.5 & 10.5 & 10.5 & 4.5 & 8.8 & 4.3 & 4.5 & 4.5 \\
\hline Grout & 0.9 & 0.9 & 0.9 & 2913 & 2764 & 2731 & 783 & 1938 \\
\hline Polymer & 246 & 246 & 246 & 1602 & 1688 & 1578 & 13.2 & 1722 \\
\hline Ceramic & 0 & 0 & 0 & 0 & 0 & 0 & 2708 & 0 \\
\hline Slag/Glass & 1519 & 1451 & 1517 & 0 & 0 & 0 & 0 & 0 \\
\hline Special Waste & 153 & 153 & 153 & 155 & 155 & 155 & 155 & 155 \\
\hline $\begin{array}{l}\text { Total Effluent (excluding } \\
\text { clean metal) }\end{array}$ & 23025 & 9219 & 13088 & 5429 & 5574 & 5471 & 5885 & 5817 \\
\hline
\end{tabular}


Table 5-2 (continued) System Effluents (lbs/hr)

\begin{tabular}{|c||c|c|c|c|c|}
\hline Effluent (lbs/hr) & $\begin{array}{c}\text { Replace Grout } \\
\text { with } \\
\text { Vitrification } \\
\text { (ENTS-1) }\end{array}$ & $\begin{array}{c}\text { Vitrify } \\
\text { Untreated } \\
\text { Sludge } \\
\text { (ENTS-2) }\end{array}$ & $\begin{array}{c}\text { Polymer } \\
\text { Stabilization } \\
\text { (ENTS-3) }\end{array}$ & $\begin{array}{c}\text { Pelletization } \\
\text { with Polymer } \\
\text { (ENTS-4) }\end{array}$ & $\begin{array}{c}\text { Increased } \\
\text { Reaction } \\
\text { Rate } \\
\text { (ENTS-5) }\end{array}$ \\
\hline Offgas & 1701 & 2537 & 1433 & 1433 & 1433 \\
\hline Wastewater & 1262 & 941 & 1233 & 1233 & 564 \\
\hline Clean Metal to Recycle & 605 & 605 & 456 & 456 & 451 \\
\hline Clean Lead & 26 & 26 & 26 & 26 & 21 \\
\hline Clean Metal & 579 & 579 & 430 & 430 & 430 \\
\hline Solids to Disposal & 2622 & 2096 & 3231 & 4306 & 3819 \\
\hline Mercury Amalgam & 6.7 & 6.5 & 4.5 & 4.5 & 4.5 \\
\hline Grout & 0 & 0 & 0 & 0 & 1938 \\
\hline Polymer & 1724 & 349 & 3071 & 4146 & 1722 \\
\hline Ceramic & 0 & 0 & 0 & 0 & 0 \\
\hline Slag/Glass & 736 & 1586 & 0 & 0 & 0 \\
\hline Special Waste & 155 & 155 & 155 & 155 & 155 \\
\hline $\begin{array}{l}\text { Total Effluent (excluding } \\
\text { clean metal) }\end{array}$ & 5585 & 5574 & 5897 & 6972 & 5816 \\
\hline
\end{tabular}


Table 5-3 Offgas Composition (lbs/hr)

\begin{tabular}{|l||c|c|c|c|c|c|c|c|}
\hline Offgas Components (lbs/hr) & $\begin{array}{c}\text { Rotary Kiln/ } \\
\text { Air } \\
(\mathrm{A}-1)\end{array}$ & $\begin{array}{c}\text { Plasma } \\
\text { Furnace } \\
(\mathrm{C}-1)\end{array}$ & $\begin{array}{c}\text { Steam } \\
\text { Reforming } \\
(\mathrm{H}-1)\end{array}$ & $\begin{array}{c}\text { Grout Debris } \\
(\mathrm{NT}-1)\end{array}$ & $\begin{array}{c}\text { Thermal } \\
\text { Desorption } \\
\text { (NT-2) }\end{array}$ & $\begin{array}{c}\text { Wash } \\
\text { (NT-3) }\end{array}$ & $\begin{array}{c}\text { Acid } \\
\text { Digestion } \\
\text { (NT-4) }\end{array}$ & $\begin{array}{c}\text { Catalytic } \\
\text { Wet } \\
\text { Oxidation } \\
\text { (NT-5) }\end{array}$ \\
\hline \hline Oxygen & 1625 & 1387 & 1489 & 29 & 36 & 55 & 10 & 31 \\
\hline Nitrogen & 14730 & 2062 & 19523 & 118 & 165 & 19 & 132 & 84 \\
\hline Nitrogen Oxide & 0 & 0 & 0 & 0.8 & 0.7 & 0.8 & 0.2 & 0.7 \\
\hline Water Vapor & 1478 & 357 & 1923 & 10 & 2 & 15 & 11 & 4 \\
\hline Carbon Dioxide & 2364 & 1569 & 3366 & 538 & 620 & 737 & 1288 & 1314 \\
\hline Undefined & 497 & 497 & 133 & 0 & 0 & 0 & 0 & 0 \\
\hline Sulfur Dioxide & 3 & 2 & 3 & 0 & 0 & 0 & 0 & 0 \\
\hline Total & 20197 & 5375 & 26301 & 696 & 823 & 827 & 1441 & 1422 \\
\hline
\end{tabular}

웅

\begin{tabular}{|l||c|c|c|c|c|}
\hline Offgas Components (lbs/hr) & $\begin{array}{c}\text { Replace Grout } \\
\text { with } \\
\text { Vitrification } \\
\text { (ENTS-1) }\end{array}$ & $\begin{array}{c}\text { Vitrify } \\
\text { Untreated } \\
\text { Sludge } \\
\text { (ENTS-2) }\end{array}$ & $\begin{array}{c}\text { Polymer } \\
\text { Stabilization } \\
\text { (ENTS-3) }\end{array}$ & $\begin{array}{c}\text { Pelletization } \\
\text { with Polymer } \\
\text { (ENTS-4) }\end{array}$ & $\begin{array}{c}\text { Increased } \\
\text { Reaction } \\
\text { Rate } \\
\text { (ENTS-5) }\end{array}$ \\
\hline Oxygen & 38 & 147 & 31 & 31 & 31 \\
\hline Nitrogen & 234 & 876 & 84 & 84 & 84 \\
\hline Nitrogen Oxide & 0.7 & 0.7 & 0.7 & 0.7 & 0.7 \\
\hline Water Vapor & 37 & 85 & 4 & 4 & 4 \\
\hline Carbon Dioxide & 1391 & 1428 & 1314 & 1314 & 1314 \\
\hline Other & 2 & 4 & 0 & 0 & 0 \\
\hline Total & 1701 & 2537 & 1433 & 1433 & 1433 \\
\hline
\end{tabular}


Table 5-4 Final Waste Form Mass sent to Disposal (lbs/hr)

\begin{tabular}{|c|c|c|c|c|c|c|c|}
\hline System & $\begin{array}{l}\text { Primary } \\
\text { Residue }^{\mathrm{a}}\end{array}$ & $\begin{array}{l}\text { Secondary } \\
\text { Residue }^{b}\end{array}$ & $\begin{array}{l}\text { Mercury } \\
\text { Amalgam }\end{array}$ & Grout & $\begin{array}{l}\text { Polymer or } \\
\text { Ceramic }^{c}\end{array}$ & Slag & $\begin{array}{l}\text { Special } \\
\text { Waste }\end{array}$ \\
\hline Rotary Kiln Air (A-1) & 1519 & 247 & 11 & 1 & 246 & 1519 & 153 \\
\hline Plasma Furnace (C-1) & 1451 & 247 & 11 & 1 & 246 & 1451 & 153 \\
\hline Steam Reforming (H-1) & 1517 & 247 & 11 & 1 & 246 & 1517 & 153 \\
\hline Grout Debris (NT-1) & 4297 & 218 & 4.5 & 2913 & 1602 & 0 & 155 \\
\hline Thermal Desorption (NT-2) & 4141 & 311 & 9 & 2764 & 1688 & 0 & 155 \\
\hline Wash (NT-3) & 4069 & 239 & 4 & 2731 & 1578 & 0 & 155 \\
\hline Acid Digestion (NT-4) & 3107 & 397 & 5 & 783 & 2721 & 0 & 155 \\
\hline Catalyzed Wet Ox (NT-5) & 3315 & 345 & 5 & 1938 & 1722 & 0 & 155 \\
\hline $\begin{array}{l}\text { Replace Grout with Vit. } \\
\text { (ENTS-1) }\end{array}$ & 2112 & 348 & 7 & 0 & 1724 & 736 & 155 \\
\hline $\begin{array}{l}\text { Vitrify Untreated Sludge } \\
\text { (ENTS-2) }\end{array}$ & 1586 & 349 & 7 & 0 & 349 & 1586 & 155 \\
\hline $\begin{array}{l}\text { Polymer Stabilization } \\
\text { (ENTS-3) }\end{array}$ & 2727 & 345 & 5 & 0 & 3071 & 0 & 155 \\
\hline $\begin{array}{l}\text { Pelletization with Polymer } \\
\text { (ENTS-4) }\end{array}$ & 3680 & 466 & 5 & 0 & 4146 & 0 & 155 \\
\hline $\begin{array}{l}\text { Increased Reaction Rate } \\
\text { (ENTS-5) }\end{array}$ & 3315 & 345 & 5 & 1938 & 1722 & 0 & 155 \\
\hline
\end{tabular}

Notes:

${ }^{\text {a }}$ Primary residue equals all primary waste stabilized in grout, polymer, or vitrified

${ }^{\mathrm{b}}$ Secondary residue equals the salt and sludge wastes generated by the processes and stabilized in polymer or phosphate bonded ceramic.

${ }^{c}$ Only system NT-4 uses phosphate bonded ceramic. 
Table 5-5 Final Waste Form Volume Sent to Disposal $\left(\mathrm{ft}^{3} / \mathrm{hr}\right)$

\begin{tabular}{|c|c|c|c|c|c|c|c|c|}
\hline System & $\begin{array}{l}\text { Primary } \\
\text { Residue }^{\text {a }} \\
\end{array}$ & $\begin{array}{c}\text { Secondary } \\
\text { Residue }^{\mathrm{b}} \\
\end{array}$ & $\begin{array}{c}\text { Mercury } \\
\text { Amalgam } \\
\end{array}$ & Grout & $\begin{array}{l}\text { Polymer or } \\
\text { Ceramic }^{c} \\
\end{array}$ & Slag & $\begin{array}{c}\text { Special } \\
\text { Waste }\end{array}$ & $\begin{array}{c}\text { Total } \\
\text { Volume }\end{array}$ \\
\hline Rotary Kiln / Air (A-1) & 8.1 & 3.1 & 0.01 & 0.01 & 3.1 & 8.1 & 2.4 & 13.6 \\
\hline Plasma Furnace (C-1) & 7.8 & 3.1 & 0.01 & 0.01 & 3.1 & 7.8 & 2.4 & 13.2 \\
\hline Steam Reforming (H-1) & 8.1 & 3.1 & 0.01 & 0.01 & 3.1 & 8.1 & 2.4 & 13.6 \\
\hline Grout Debris (NT-1) & 37.7 & 2.3 & 0.006 & 23 & 17 & 0 & 2.4 & 42.5 \\
\hline Thermal Desorption (NT-2) & 36.5 & 3.3 & 0.012 & 21.8 & 18 & 0 & 2.4 & 42.2 \\
\hline Wash (NT-3) & 35.8 & 2.6 & 0.001 & 21.6 & 16.8 & 0 & 2.4 & 40.8 \\
\hline Acid Digestion (NT-4) & 30.9 & 4.3 & 0.006 & 6.2 & 28.9 & 0 & 2.4 & 37.5 \\
\hline Catalyzed Wet Ox (NT-5) & 30 & 3.7 & 0.006 & 15.3 & 18.3 & 0 & 2.4 & 36 \\
\hline $\begin{array}{l}\text { Replace Grout with Vit. } \\
\text { (ENTS-1) }\end{array}$ & 18.6 & 3.7 & 0.01 & 0 & 18.3 & 3.9 & 2.4 & 24.7 \\
\hline $\begin{array}{l}\text { Vitrify Untreated Sludge } \\
\text { (ENTS-2) }\end{array}$ & 8.5 & 3.7 & 0.01 & 0 & 3.7 & 8.5 & 2.4 & 14.6 \\
\hline $\begin{array}{l}\text { Polymer Stabilization } \\
\text { (ENTS-3) }\end{array}$ & 29 & 3.7 & 0.01 & 0 & 32.7 & 0 & 2.4 & 35.1 \\
\hline $\begin{array}{l}\text { Pelletization with Polymer } \\
\text { (ENTS-4) }\end{array}$ & 39.2 & 5 & 0.01 & 0 & 44.1 & 0 & 2.4 & 46.5 \\
\hline $\begin{array}{l}\text { Increased Reaction Rate } \\
\text { (ENTS-5) }\end{array}$ & 30 & 3.7 & 0.01 & 15.3 & 18.3 & 0 & 2.4 & 36 \\
\hline
\end{tabular}

Notes:

${ }^{a}$ Primary residue equals all primary waste stabilized in grout, polymer, or vitrified.

${ }^{b}$ Secondary residue equals the salt and sludge wastes generated by the processes and stabilized in polymer or phosphate bonded ceramic.

${ }^{c}$ Only system NT-4 uses phosphate bonded ceramic. 
Table 5-6 Waste Volume Reduction

\begin{tabular}{|l|c|c|}
\hline \multicolumn{1}{|c||}{ System } & Volume In/Volume Out & $\begin{array}{c}\text { Volume Out as a Percent } \\
\text { of Volume In }\end{array}$ \\
\hline \hline Rotary Kiln /Air (A-1) & 3.37 & $30 \%$ \\
\hline Plasma Furnace (C-1) & 3.46 & $29 \%$ \\
\hline Steam Reforming (H-1) & 3.437 & $30 \%$ \\
\hline Grout Debris (NT-1) & 1.05 & $95 \%$ \\
\hline Thermal Desorption (NT-2) & 1.06 & $94 \%$ \\
\hline Wash (NT-3) & 1.1 & $91 \%$ \\
\hline Acid Digestion (NT-4) & 1.19 & $84 \%$ \\
\hline Catalyzed Wet Ox (NT-5) & 1.24 & $81 \%$ \\
\hline $\begin{array}{l}\text { Replace Grout with Vit. } \\
\text { (ENTS-1) }\end{array}$ & 1.81 & $55 \%$ \\
\hline $\begin{array}{l}\text { Vitrify Untreated Sludge } \\
\text { (ENTS-2) }\end{array}$ & 3.06 & $33 \%$ \\
\hline $\begin{array}{l}\text { Polymer Stabilization } \\
\text { (ENTS-3) }\end{array}$ & 1.28 & $78 \%$ \\
\hline $\begin{array}{l}\text { Pelletization with Polymer } \\
\text { (ENTS-4) }\end{array}$ & 0.96 & $104 \%$ \\
\hline $\begin{array}{l}\text { Increased Reaction Rate } \\
\text { (ENTS-5) }\end{array}$ & 1.24 & $81 \%$ \\
\hline
\end{tabular}


Table 5-7 Treatment System Energy Cost

\begin{tabular}{|l||c|c|c|c|}
\hline \multicolumn{1}{|c||}{ System } & \multicolumn{3}{c|}{ Energy Costs per Hour } & \multirow{2}{*}{$\begin{array}{c}\text { Fraction of } \\
\text { Treatment Cost }\end{array}$} \\
\cline { 2 - 4 } & Electrical Energy & Natural Gas & Total Energy Cost & 0.00176 \\
\hline \hline Rotary Kiln / Air (A-1) & $\$ 29.36$ & $\$ 17.97$ & $\$ 47.33$ & 0.00666 \\
\hline Plasma Furnace (C-1) & $\$ 163.54$ & $\$ 0.00$ & $\$ 163.54$ & 0.00185 \\
\hline Steam Reforming (H-1) & $\$ 42.59$ & $\$ 7.83$ & $\$ 50.42$ & 0.00193 \\
\hline Grout Debris (NT-1) & $\$ 67.98$ & $\$ 1.25$ & $\$ 69.23$ & 0.00008 \\
\hline Thermal Desorption (NT-2) & $\$ 0.38$ & $\$ 2.41$ & $\$ 2.79$ & 0.00253 \\
\hline Wash (NT-3) & $\$ 93.52$ & $\$ 0.37$ & $\$ 93.89$ & 0.00005 \\
\hline Acid Digestion (NT-4) & $\$ 0.61$ & $\$ 1.48$ & $\$ 2.09$ & 0.00005 \\
\hline Catalyzed Wet Ox (NT-5) & $\$ 0.59$ & $\$ 1.30$ & $\$ 1.89$ & 0.00094 \\
\hline $\begin{array}{l}\text { Replace Grout with Vit. } \\
\text { (ENTS-1) }\end{array}$ & $\$ 37.60$ & $\$ 1.21$ & $\$ 38.81$ & 0.00125 \\
\hline $\begin{array}{l}\text { Vitrify Untreated Sludge } \\
\text { (ENTS-2) }\end{array}$ & $\$ 46.44$ & $\$ 0.18$ & $\$ 46.62$ & 0.00007 \\
\hline $\begin{array}{l}\text { Polymer Stabilization } \\
\text { (ENTS-3) }\end{array}$ & $\$ 1.59$ & $\$ 1.26$ & $\$ 2.85$ & 0.00007 \\
\hline $\begin{array}{l}\text { Pelletization with Polymer } \\
\text { (ENTS-4) }\end{array}$ & $\$ 1.59$ & $\$ 1.32$ & $\$ 2.91$ & 0.00005 \\
\hline $\begin{array}{l}\text { Increased Reaction Rate } \\
\text { (ENTS-5) }\end{array}$ & $\$ 0.59$ & $\$ 1.30$ & $\$ 1.89$ & \\
\hline
\end{tabular}


Table 5-8 Reagent Use (lbs/hr)

\begin{tabular}{|l||c|c|c|c|c|c|}
\hline Reagents (lbs/hr) & $\begin{array}{c}\text { All Thermal } \\
\text { Systems }\end{array}$ & $\begin{array}{c}\text { Grout Debris } \\
\text { (NT-1) }\end{array}$ & $\begin{array}{c}\text { Thermal } \\
\text { Desorption } \\
(\mathrm{NT}-2)\end{array}$ & $\begin{array}{c}\text { Wash } \\
\text { (NT-3) }\end{array}$ & $\begin{array}{c}\text { Acid } \\
\text { Digestion } \\
\text { (NT-4) }\end{array}$ & $\begin{array}{c}\text { Catalytic Wet } \\
\text { Oxidation } \\
\text { (NT-5) }\end{array}$ \\
\hline \hline Copper & 5 & 1.9 & 3.6 & 1.8 & 1.8 & 1.9 \\
\hline Sodium Hydroxide & 78 & 58 & 84.7 & 58.5 & 104 & 93.4 \\
\hline CWO Acid Solution & 0 & 0 & 9.7 & 0 & 0 & 24.5 \\
\hline Ferric Chloride & & & 5.8 & & & 14.7 \\
\hline Hydrochloric Acid & & & 1.1 & & & 2.8 \\
\hline Water & & & 2.8 & & & 7.0 \\
\hline Ammonia & 0 & 4 & 0 & 5.5 & 6.8 & 0 \\
\hline Nitric Acid & 0 & 21.4 & 0 & 28.8 & 73.7 & 0 \\
\hline Silver Nitrate & 0 & 2.9 & 0 & 4.7 & 0 & 0 \\
\hline Hydrogen Peroxide & 0 & 29.2 & 0.6 & 48.6 & 0.6 & 0.6 \\
\hline
\end{tabular}

\begin{tabular}{|l||c|c|c|c|c|}
\hline Reagents (lbs/hr) & $\begin{array}{c}\text { Replace Grout } \\
\text { with } \\
\text { Vitrification } \\
\text { (ENTS-1) }\end{array}$ & $\begin{array}{c}\text { Vitrify } \\
\text { Untreated } \\
\text { Sludge } \\
\text { (ENTS-2) }\end{array}$ & $\begin{array}{c}\text { Polymer } \\
\text { Stabilization } \\
\text { (ENTS-3) }\end{array}$ & $\begin{array}{c}\text { Pelletization } \\
\text { with Polymer } \\
\text { (ENTS-4) }\end{array}$ & $\begin{array}{c}\text { Increased } \\
\text { Reaction } \\
\text { Rate } \\
\text { (ENTS-5) }\end{array}$ \\
\hline \hline Copper & 2.8 & 2.7 & 1.9 & 1.9 & 1.9 \\
\hline Sodium Hydroxide & 94.2 & 94.1 & 93.4 & 93.4 & 93.4 \\
\hline CWO Acid Solution & 24.5 & 23.7 & 24.5 & 24.5 & 24.5 \\
\hline Ferric Chloride & 14.7 & 14.7 & 14.2 & 14.7 & 14.7 \\
\hline Hydrochloric Acid & 2.8 & 2.8 & 2.8 & 2.8 & 2.8 \\
\hline Water & 7.0 & 7.0 & 6.7 & 7.0 & 7.0 \\
\hline Ammonia & 0 & 0 & 0 & 0 & 0 \\
\hline Nitric Acid & 0 & 0 & 0 & 0 & 0 \\
\hline Silver Nitrate & 0 & 0 & 0 & 0 & 0 \\
\hline Hydrogen Peroxide & 0.6 & 0.6 & 0.6 & 0.7 & 0.6 \\
\hline
\end{tabular}


Table 5-9 Truck Traffic (trucks per year)

\begin{tabular}{|c|c|c|c|c|c|c|c|}
\hline System & $\begin{array}{l}\text { Incoming } \\
\text { Waste }\end{array}$ & $\begin{array}{l}\text { Incoming } \\
\text { Reagent }\end{array}$ & $\begin{array}{l}\text { Stabilizing } \\
\text { Agents }\end{array}$ & $\begin{array}{c}\text { Total } \\
\text { Incoming } \\
\text { Truck Traffic }\end{array}$ & $\begin{array}{l}\text { Waste to } \\
\text { Disposal }\end{array}$ & $\begin{array}{l}\text { Metals/Lead } \\
\text { to Storage }\end{array}$ & $\begin{array}{l}\text { Total Outgoing } \\
\text { Loaded Trucks }\end{array}$ \\
\hline Rotary Kiln / Air (A-1) & 269 & 14 & 58 & 341 & 177 & 57 & 234 \\
\hline Plasma Furnace (C-1) & 269 & 14 & 56 & 339 & 171 & 60 & 231 \\
\hline Steam Reforming (H-1) & 269 & 14 & 58 & 341 & 177 & 57 & 234 \\
\hline Grout Debris (NT-1) & 269 & 17 & 198 & 484 & 428 & 41 & 470 \\
\hline Thermal Desorption (NT-2) & 269 & 16 & 196 & 480 & 423 & 41 & 464 \\
\hline Wash (NT-3) & 269 & 21 & 189 & 479 & 409 & 41 & 451 \\
\hline Acid Digestion (NT-4) & 269 & 27 & 158 & 454 & 336 & 41 & 377 \\
\hline Catalyzed Wet Ox (NT-5) & 269 & 18 & 162 & 449 & 350 & 41 & 391 \\
\hline $\begin{array}{l}\text { Replace Grout with Vit. } \\
\text { (ENTS-1) }\end{array}$ & 269 & 19 & 108 & 395 & 240 & 55 & 296 \\
\hline Vitrify Untreated Sludge (ENTS-2) & 269 & 18 & 64 & 352 & 192 & 55 & 248 \\
\hline Polymer Stabilization (ENTS-3) & 269 & 18 & 141 & 428 & 296 & 42 & 338 \\
\hline Pelletization with Polymer (ENTS-4) & 269 & 18 & 239 & 526 & 395 & 42 & 436 \\
\hline Increased Reaction Rate (ENTS-5) & 269 & 18 & 162 & 449 & 350 & 41 & 391 \\
\hline
\end{tabular}

Notes:

a. Primary residue equals all primary waste stabilized in grout, polymer, or vitrified.

b. Secondary residue equals the salt and sludge wastes generated by the processes and stabilized in polymer or phosphate bonded ceramic.

c. Only system NT-4 uses phosphate bonded ceramic. 
Table 5-10 Personnel Requirements by Subsystem (Full Time Equivalents)

\begin{tabular}{|c|c|c|c|c|c|c|c|c|}
\hline Subsystems & $\begin{array}{c}\text { Rotary Kiln/ } \\
\text { Air } \\
\text { (A-1) } \\
\end{array}$ & $\begin{array}{l}\text { Plasma } \\
\text { Furnace } \\
\text { (C-1) }\end{array}$ & $\begin{array}{c}\text { Steam } \\
\text { Reforming } \\
(\mathrm{H}-1)\end{array}$ & $\begin{array}{l}\text { Grout Debris } \\
\text { (NT-1) }\end{array}$ & $\begin{array}{l}\text { Thermal } \\
\text { Desorption } \\
\text { (NT-2) }\end{array}$ & $\begin{array}{l}\text { Wash } \\
\text { (NT-3) }\end{array}$ & $\begin{array}{c}\text { Acid } \\
\text { Digestion } \\
\text { (NT-4) }\end{array}$ & $\begin{array}{c}\text { Catalytic Wet } \\
\text { Oxidation } \\
\text { (NT-5) } \\
\end{array}$ \\
\hline Administration & 27 & 27 & 27 & 32 & 32 & 32 & 32 & 32 \\
\hline Receiving and Preparation & 112 & 112 & 132 & 170 & 170 & 170 & 170 & 170 \\
\hline Organic Destruction & 16 & 16 & 20 & 12 & 12 & 12 & 30 & 26 \\
\hline Air Pollution Control & 4 & 4 & 4 & 4 & 4 & 4 & 4 & 4 \\
\hline Primary Stabilization & 16 & 16 & 16 & 8 & 8 & 8 & 45 & 8 \\
\hline Secondary Stabilization & 8 & 8 & 8 & 38 & 38 & 40 & 5 & 38 \\
\hline Metal Melting & 5 & $\mathbf{a}$ & 5 & $\mathrm{~b}$ & $\mathrm{~b}$ & $\mathrm{~b}$ & $\mathrm{~b}$ & $\mathrm{~b}$ \\
\hline Metal Decontamination & 5 & 5 & 5 & 4 & 4 & 4 & 4 & 4 \\
\hline Lead Recovery & 8 & 8 & 8 & 3 & 3 & 3 & 3 & 3 \\
\hline Mercury Amalgamation & 4 & 4 & 4 & 1 & 1 & 1 & 1 & 1 \\
\hline Process Residue/Soil & ${ }^{\circ}$ & ${ }^{\circ}$ & $d$ & 10 & 16 & 3 & 10 & 10 \\
\hline Debris Treatment & ${ }^{\circ}$ & ${ }^{\circ}$ & d & $\mathrm{e}$ & $\mathbf{f}$ & 16 & 8 & 8 \\
\hline Aqueous Waste Treatment & 16 & 16 & 16 & 5 & 5 & 5 & 5 & 5 \\
\hline Special Waste Treatment & 2 & 2 & 2 & 3 & 3 & 3 & 3 & 3 \\
\hline Certification \& Shipping & 32 & 32 & 32 & 106 & 105 & 110 & 87 & 88 \\
\hline Support & 5 & 5 & 5 & 5 & 5 & 5 & 5 & 5 \\
\hline Total & 260 & 255 & 284 & 401 & 406 & 416 & 412 & 405 \\
\hline
\end{tabular}

Notes:

anternally contaminated ferrous metal is treated in the plasma furnace.

${ }^{b}$ This subsystem is not provided in this system.

'These waste streams are treated in the thermal organic destruction subsystem

${ }^{d}$ These waste streams are treated in primary stabilization (vitrifier).

Untreated debris is stabilized.

Debris is thermally desorbed with process residue/soil.

${ }^{g}$ Only one method of stabilization is provided in this system 
Table 5-10 (continued) Personnel Requirements by Subsystem (Full Time Equivalents)

\begin{tabular}{|l||c|c|c|c|c|}
\hline \multicolumn{1}{|c|}{ Subsystems } & $\begin{array}{c}\text { Replace Grout } \\
\text { with } \\
\text { Vitrification } \\
\text { (ENTS-1) }\end{array}$ & $\begin{array}{c}\text { Vitrify } \\
\text { Untreated } \\
\text { Sludge } \\
\text { (ENTS-2) }\end{array}$ & $\begin{array}{c}\text { Polymer } \\
\text { Stabilization } \\
\text { (ENTS-3) }\end{array}$ & $\begin{array}{c}\text { Pelletization } \\
\text { with Polymer }\end{array}$ & $\begin{array}{c}\text { Increased } \\
\text { Reaction } \\
\text { Rate } \\
\text { (ENTS-4) }\end{array}$ \\
\hline Administration & 32 & 32 & 32 & 32 & 32 \\
\hline Receiving and Preparation & 170 & 170 & 170 & 170 & 170 \\
\hline Organic Destruction & 26 & 26 & 26 & 26 & 15 \\
\hline Air Pollution Control & 4 & 4 & 4 & 4 & 4 \\
\hline Primary Stabilization & 16 & 18 & 56 & 45 & 8 \\
\hline Secondary Stabilization & 38 & 8 & 8 & 8 & 38 \\
\hline Metal Melting & 5 & 5 & 6 & b & b \\
\hline Metal Decontamination & 4 & 4 & 4 & 4 & 4 \\
\hline Lead Recovery & 8 & 8 & 3 & 3 & 3 \\
\hline Mercury Amalgamation & 4 & 4 & 1 & 1 & 1 \\
\hline Process Residue/Soil & 10 & 2 & 10 & 10 & 10 \\
\hline Debris Treatment & 8 & 8 & 8 & 8 & 8 \\
\hline Aqueous Waste Treatment & 5 & 5 & 5 & 5 & 5 \\
\hline Special Waste Treatment & 3 & 3 & 3 & 3 & 3 \\
\hline Certification \& Shipping & 67 & 37 & 88 & 175 & 88 \\
\hline Support Systems & 5 & 5 & 5 & 5 & 5 \\
\hline Total & 405 & 339 & 415 & 491 & 394 \\
\hline
\end{tabular}

Notes:

anternally contaminated ferrous metal is treated in the plasma furnace.

${ }^{b}$ This subsystem is not provided in this system.

${ }^{c}$ These waste streams are treated in the thermal organic destruction subsystem

${ }^{\mathrm{d}}$ These waste streams are treated in primary stabilization (vitrifier).

${ }^{e}$ Untreated debris is stabilized.

Debris is thermally desorbed with process residue/soil.

${ }^{8}$ Only one method of stabilization is provided in this system 
Table 5-11 Total Life-Cycle Costs (\$ million)

\begin{tabular}{|c|c|c|c|c|c|c|c|c|c|c|c|c|c|c|c|c|}
\hline \multirow[t]{2}{*}{$\begin{array}{c}\text { Cost } \\
\text { Component }\end{array}$} & \multicolumn{2}{|c|}{$\begin{array}{c}\text { Rotary Kiln /Air } \\
\text { (A-1) }\end{array}$} & \multicolumn{2}{|c|}{$\begin{array}{l}\text { Plasma Furnace } \\
(\text { (C-1) }\end{array}$} & \multicolumn{2}{|c|}{$\begin{array}{c}\text { Steam } \\
\text { Reforming } \\
(\mathrm{H}-1)\end{array}$} & \multicolumn{2}{|c|}{$\begin{array}{l}\text { Grout Debris } \\
\text { (NT-1) }\end{array}$} & \multicolumn{2}{|c|}{$\begin{array}{c}\text { Thermal } \\
\text { Desorption } \\
\text { (NT-2) }\end{array}$} & \multicolumn{2}{|c|}{$\begin{array}{l}\text { Wash } \\
\text { (NT-3) }\end{array}$} & \multicolumn{2}{|c|}{$\begin{array}{l}\text { Acid Digestion } \\
(\mathrm{NT}-4)\end{array}$} & \multicolumn{2}{|c|}{$\begin{array}{l}\text { Catalytic Wet } \\
\text { Oxidation } \\
\text { (NT-5) }\end{array}$} \\
\hline & Cost & $\%$ & Cost & $\%$ & Cost & $\%$ & Cost & $\%$ & Cost & $\%$ & Cost & $\%$ & Cost & $\%$ & 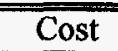 & $\overline{0 \%}$ \\
\hline $\begin{array}{l}\text { Test \& } \\
\text { Demonstration }\end{array}$ & $\$ 100$ & $4 \%$ & $\$ 99$ & $4 \%$ & $\$ 62$ & $3 \%$ & $\$ 134$ & $4 \%$ & $\$ 142$ & $4 \%$ & $\$ 143$ & $4 \%$ & $\$ 208$ & $5 \%$ & $\$ 167$ & $\overline{4 \%}$ \\
\hline $\begin{array}{l}\text { Operations } \\
\text { Budget }\end{array}$ & $\$ 97$ & $4 \%$ & $\$ 90$ & $4 \%$ & $\$ 99$ & $4 \%$ & $\$ 131$ & $4 \%$ & $\$ 129$ & $4 \%$ & $\$ 135$ & $4 \%$ & $\$ 138$ & $4 \%$ & $\$ 139$ & $4 \%$ \\
\hline Capital & $\$ 554$ & $23 \%$ & $\$ 459$ & $20 \%$ & $\$ 588$ & $24 \%$ & $\$ 600$ & $16 \%$ & $\$ 561$ & $15 \%$ & $\$ 632$ & $17 \%$ & $\$ 673$ & $17 \%$ & $\$ 668$ & $18 \%$ \\
\hline $\mathrm{O \& M}$ & $\$ 1,362$ & $56 \%$ & $\$ 1,283$ & $57 \%$ & $\$ 1,390$ & $57 \%$ & $\$ 1,950$ & $52 \%$ & $\$ 1,930$ & $53 \%$ & $\$ 2,005$ & $53 \%$ & $\$ 2,040$ & $53 \%$ & $\$ 2,058$ & $54 \%$ \\
\hline$\overline{\mathrm{D} \& \mathrm{D}}$ & $\$ 54$ & $2 \%$ & $\$ 50$ & $2 \%$ & $\$ 54$ & $2 \%$ & $\$ 74$ & $2 \%$ & $\$ 69$ & $2 \%$ & $\$ 77$ & $2 \%$ & $\$ 80$ & $2 \%$ & $\$ 78$ & $2 \%$ \\
\hline Disposal & $\$ 266$ & $11 \%$ & $\$ 259$ & $12 \%$ & $\$ 266$ & $11 \%$ & $\$ 833$ & $22 \%$ & $\$ 827$ & $23 \%$ & $\$ 799$ & $21 \%$ & $\$ 736$ & $19 \%$ & $\$ 707$ & $19 \%$ \\
\hline Total & $\$ 2,433$ & & $\$ 2,240$ & & $\$ 2,459$ & & $\$ 3,722$ & & $\$ 3,658$ & & $\$ 3,792$ & & $\$ 3,875$ & & $\$ 3,817$ & \\
\hline & & & & & & & & & & & & & & & & \\
\hline $\begin{array}{l}\text { Total Without } \\
\text { Disposal }\end{array}$ & $\$ 2,167$ & & $\$ 1,981$ & & $\$ 2,193$ & & $\$ 2,889$ & & $\$ 2,831$ & & $\$ 2,993$ & & $\$ 3,139$ & & $\$ 3,110$ & \\
\hline $\begin{array}{l}\text { Unit } \\
\text { Treatment } \\
\text { Costs With } \\
\text { Disposal }\end{array}$ & $\$ 7.85$ & & $\$ 7.17$ & & $\$ 8.90$ & & $\$ 15.77$ & & $\$ 15.50$ & & $\$ 16.06$ & & $\$ 16.42$ & & $\$ 16.17$ & \\
\hline $\begin{array}{l}\text { Unit } \\
\text { Treatment } \\
\text { Costs Without } \\
\text { Disposal }\end{array}$ & $\$ 9.18$ & & $\$ 8.39$ & & $\$ 9.29$ & & $\$ 12.24$ & & $\$ 11.99$ & & $\$ 12.68$ & & $\$ 13.30$ & & 13.18 & \\
\hline
\end{tabular}


Table 5-11 (continued) Total Life-Cycle Costs (\$ million)

\begin{tabular}{|l||c|c|c|c|c|c|c|c|c|c|}
\hline \multicolumn{1}{|c|}{$\begin{array}{c}\text { Cost } \\
\text { Component }\end{array}$} & $\begin{array}{c}\text { Replace Grout } \\
\text { with } \\
\text { Vitrification } \\
\text { (ENTS-1) }\end{array}$ & \multicolumn{2}{c|}{$\begin{array}{c}\text { Vitrify } \\
\text { Untreated } \\
\text { Sludge } \\
\text { (ENTS-2) }\end{array}$} & \multicolumn{2}{c|}{$\begin{array}{c}\text { Polymer } \\
\text { Stabilization } \\
\text { (ENTS-3) }\end{array}$} & $\begin{array}{c}\text { Pelletization } \\
\text { with Polymer } \\
\text { (ENTS-4) }\end{array}$ & \multicolumn{2}{c|}{$\begin{array}{c}\text { Increased } \\
\text { Reaction Rate } \\
\text { (ENTS-5) }\end{array}$} \\
\hline \hline $\begin{array}{l}\text { Test \& } \\
\text { Demonstration }\end{array}$ & $\$ 167$ & $4 \%$ & $\$ 148$ & $5 \%$ & $\$ 157$ & $4 \%$ & $\$ 157$ & $4 \%$ & $\$ 167$ & $4 \%$ \\
\hline Operations Budget & $\$ 147$ & $4 \%$ & $\$ 132$ & $4 \%$ & $\$ 142$ & $4 \%$ & $\$ 161$ & $4 \%$ & $\$ 138$ & $4 \%$ \\
\hline Capital & $\$ 795$ & $21 \%$ & $\$ 762$ & $23 \%$ & $\$ 660$ & $17 \%$ & $\$ 669$ & $15 \%$ & $\$ 675$ & $18 \%$ \\
\hline O\&M & $\$ 2,134$ & $56 \%$ & $\$ 1,878$ & $57 \%$ & $\$ 2,108$ & $55 \%$ & $\$ 2450$ & $55 \%$ & 2023 & $53 \%$ \\
\hline D\&D & $\$ 84$ & $2 \%$ & $\$ 81$ & $2 \%$ & $\$ 78$ & $2 \%$ & $\$ 78$ & $2 \%$ & $\$ 76$ & $2 \%$ \\
\hline Disposal & $\$ 484$ & $13 \%$ & $\$ 286$ & $9 \%$ & $\$ 688$ & $18 \%$ & $\$ 911$ & $21 \%$ & $\$ 707$ & $19 \%$ \\
\hline Total & $\$ 3,810$ & & $\$ 3,287$ & & $\$ 3,832$ & & $\$ 4,425$ & & $\$ 3,785$ & \\
\hline & & & & & & & & & & \\
\hline $\begin{array}{l}\text { Total Without } \\
\text { Disposal }\end{array}$ & $\$ 3,326$ & & $\$ 3,001$ & & $\$ 3,144$ & & $\$ 3,514$ & & $\$ 3,078$ & \\
\hline & & & & & & & & & & \\
\hline $\begin{array}{l}\text { Unit Treatment } \\
\text { Costs With } \\
\text { Disposal }\end{array}$ & $\$ 16.14$ & & $\$ 13.93$ & & $\$ 16.24$ & & $\$ 18.75$ & & $\$ 16.04$ & \\
\hline & & & & & & & & & & \\
\hline $\begin{array}{l}\text { Unit Treatment } \\
\text { Costs Without } \\
\text { Disposal }\end{array}$ & $\$ 14.09$ & & $\$ 12.72$ & & $\$ 13.32$ & & $\$ 14.89$ & & $\$ 13.04$ & \\
\hline
\end{tabular}


Table 5-12 Subsystem Life-Cycle Cost (\$ million)

\begin{tabular}{|c|c|c|c|c|c|c|c|c|c|c|c|c|c|c|c|c|}
\hline \multirow[t]{2}{*}{ Subsystem } & \multicolumn{2}{|c|}{$\begin{array}{l}\text { Rotary Kiln /Air } \\
\text { (A-1) }\end{array}$} & \multicolumn{2}{|c|}{$\begin{array}{l}\text { Plasma Furnace } \\
\text { (C-1) }\end{array}$} & \multicolumn{2}{|c|}{$\begin{array}{c}\text { Steam } \\
\text { Reforming } \\
(\mathrm{H}-1) \\
\end{array}$} & \multicolumn{2}{|c|}{$\begin{array}{l}\text { Grout Debris } \\
\text { (NT-1) }\end{array}$} & \multicolumn{2}{|c|}{$\begin{array}{c}\text { Thermal } \\
\text { Desorption } \\
\text { (NT-2) }\end{array}$} & \multicolumn{2}{|c|}{$\begin{array}{c}\text { Wash } \\
\text { (NT-3) }\end{array}$} & \multicolumn{2}{|c|}{$\begin{array}{l}\text { Acid Digestion } \\
(\text { NT-4) }\end{array}$} & \multicolumn{2}{|c|}{$\begin{array}{c}\text { Catalytic Wet } \\
\text { Oxidation } \\
\text { (NT-5) }\end{array}$} \\
\hline & $\frac{\text { Cost }}{8667}$ & $\%$ & Cost & $\%$ & Cost & $\%$ & Cost & $\%$ & Cost & $\%$ & Cost & $\%$ & Cost & $\%$ & Cost & $\%$ \\
\hline $\begin{array}{l}\text { Front-End } \\
\text { Handling }\end{array}$ & $\$ 667$ & $27 \%$ & $\$ 660$ & $29 \%$ & $\$ 783$ & $32 \%$ & $\$ 941$ & $25 \%$ & $\$ 941$ & $26 \%$ & $\$ 941$ & $25 \%$ & $\$ 941$ & $24 \%$ & $\$ 941$ & $25 \%$ \\
\hline Organic & $\$ 162$ & $7 \%$ & $\$ 202$ & $9 \%$ & $\$ 185$ & $8 \%$ & $\$ 246$ & $7 \%$ & $\$ 177$ & $5 \%$ & $\$ 246$ & $6 \%$ & $\$ 391$ & $10 \%$ & & 108 \\
\hline $\begin{array}{l}\text { Destruction } \\
\text { Thermal }\end{array}$ & & & & & & & & & & & & & & $10 \%$ & $\$ 396$ & $10 \$$ \\
\hline$\frac{\text { Desorption }}{\text { Wash }}$ & & (3. & & & & & $\$ 134$ & $4 \%$ & $\$ 157$ & $4 \%$ & & & $\$ 127$ & $3 \%$ & $\$ 127$ & $3 \%$ \\
\hline$\frac{\text { Wash }}{\text { Primary }}$ & $\$ 162$ & $7 \%$ & & $\frac{3}{00 \%}$ & 0.05 & & & $10 x$ & & & $\$ 214$ & $6 \%$ & $\$ 153$ & $4 \%$ & $\$ 153$ & $4 \%$ \\
\hline Treatment & $\$ 104$ & $1 \%$ & $\$ 202$ & $9 \%$ & $\$ 185$ & $8 \%$ & $\$ 380$ & $10 \%$ & $\$ 334$ & $9 \%$ & $\$ 460$ & $12 \%$ & $\$ 670$ & $17 \%$ & $\$ 676$ & $18 \%$ \\
\hline & & & & 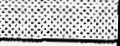 & & 18 & & 3. & & & & & & & & \\
\hline $\begin{array}{l}\text { Aqueous } \\
\text { Waste }\end{array}$ & $\$ 149$ & $6 \%$ & $\$ 149$ & $7 \%$ & $\$ 54$ & $2 \%$ & $\$ 150$ & $4 \%$ & $\$ 150$ & $4 \%$ & $\$ 150$ & $4 \%$ & $\$ 150$ & $4 \%$ & $\$ 150$ & $4 \%$ \\
\hline APC & $\$ 115$ & $5 \%$ & $\$ 80$ & $4 \%$ & $\$ 88$ & $4 \%$ & $\$ 70$ & $2 \%$ & $\$ 70$ & $2 \%$ & $\$ 70$ & $2 \%$ & $\$ 70$ & $2 \%$ & $\$ 70$ & $2 \%$ \\
\hline Stabilization & $\$ 377$ & $15 \%$ & $\$ 284$ & $13 \%$ & $\$ 377$ & $15 \%$ & $\$ 444$ & 125 & $\$ 444$ & $12 \%$ & $\$ 452$ & $12 \%$ & $\$ 480$ & $12 \%$ & $\$ 444$ & $12 \%$ \\
\hline $\begin{array}{l}\text { Metal } \\
\text { Recovery }\end{array}$ & $\$ 235$ & $10 \%$ & $\$ 143$ & $6 \%$ & $\$ 235$ & $10 \%$ & $\$ 105$ & $3 \%$ & $\$ 105$ & $3 \%$ & $\$ 105$ & $3 \%$ & $\$ 105$ & $3 \%$ & $\$ 105$ & $3 \%$ \\
\hline Special Waste & $\$ 29$ & $1 \%$ & $\$ 29$ & $1 \%$ & $\$ 29$ & $1 \%$ & $\$ 46$ & $1 \%$ & $\$ 46$ & $1 \%$ & $\$ 46$ & $1 \%$ & $\$ 46$ & $1 \%$ & $\$ 46$ & $1 \%$ \\
\hline Certify \& Ship & $\$ 193$ & $8 \%$ & $\$ 193$ & $9 \%$ & $\$ 193$ & $8 \%$ & $\$ 499$ & $13 \%$ & $\$ 491$ & $13 \%$ & $\$ 514$ & $14 \%$ & $\$ 420$ & $11 \%$ & $\$ 424$ & $11 \%$ \\
\hline $\begin{array}{l}\text { Admin \& } \\
\text { Support } \\
\end{array}$ & $\$ 240$ & $10 \%$ & $\$ 240$ & $11 \%$ & $\$ 249$ & $10 \%$ & $\$ 254$ & $7 \%$ & $\$ 250$ & $7 \%$ & $\$ 254$ & $7 \%$ & $\$ 256$ & $7 \%$ & $\$ 254$ & $7 \%$ \\
\hline Disposal & $\$ 266$ & $11 \%$ & $\$ 259$ & $12 \%$ & $\$ 266$ & $11 \%$ & $\$ 833$ & $22 \%$ & $\$ 827$ & $23 \%$ & $\$ 799$ & $21 \%$ & $\$ 736$ & $19 \%$ & $\$ 707$ & $19 \%$ \\
\hline Tatal & & . & & & & & & & & & & & & & & \\
\hline Total & $\$ 2,434$ & & $\$ 2,239$ & & $\$ 2,460$ & & $\$ 3,721$ & & $\$ 3,657$ & & $\$ 3,790$ & & $\$ 3,874$ & & $\$ 3,816$ & \\
\hline
\end{tabular}


Table 5-12 (continued) Subsystem Life-Cycle Costs (\$millions)

\begin{tabular}{|c|c|c|c|c|c|c|c|c|c|c|}
\hline $\begin{array}{c}\text { Cost } \\
\text { Component }\end{array}$ & $\begin{array}{r}\text { Replac } \\
\text { w } \\
\text { Vitrif } \\
\text { (EN }\end{array}$ & $\begin{array}{l}\text { Grout } \\
\text { ation } \\
\text {-1) }\end{array}$ & $\begin{array}{r}\mathrm{V} \\
\text { Unt } \\
\text { S1 } \\
\text { (EN }\end{array}$ & $\begin{array}{l}\text { ify } \\
\text { ated } \\
\text { lge } \\
\text { S-2) }\end{array}$ & $\begin{array}{r}\text { Po } \\
\text { Stab } \\
\text { (E) }\end{array}$ & $\begin{array}{l}\text { ner } \\
\text { zation } \\
\text { S-3) }\end{array}$ & $\begin{array}{l}\text { Pelle } \\
\text { with } \\
\text { (EN }\end{array}$ & $\begin{array}{l}\text { ation } \\
\text { lymer } \\
\text { s-4) }\end{array}$ & $\begin{array}{r}\text { Inc } \\
\text { React } \\
\text { (E) }\end{array}$ & $\begin{array}{l}\text { ased } \\
\text { n Rate } \\
\text { S-5) }\end{array}$ \\
\hline & Cost & $\%$ & Cost & $\%$ & Cost & $\%$ & Cost & $\%$ & Cost & $\%$ \\
\hline $\begin{array}{l}\text { Front-End } \\
\text { Handling }\end{array}$ & $\$ 941$ & $25 \%$ & $\$ 941$ & $29 \%$ & $\$ 941$ & $24 \%$ & $\$ 941$ & $21 \%$ & $\$ 941$ & $25 \%$ \\
\hline & & : & 2.1 .8 & : & & & . & (2). & Fis & 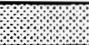 \\
\hline $\begin{array}{l}\text { Organic } \\
\text { Destruction }\end{array}$ & $\$ 396$ & $10 \%$ & $\$ 396$ & $12 \%$ & $\$ 396$ & $10 \%$ & $\$ 396$ & $9 \%$ & $\$ 359$ & $9 \%$ \\
\hline $\begin{array}{l}\text { Thermal } \\
\text { Desorption }\end{array}$ & $\$ 127$ & $3 \%$ & & & $\$ 127$ & $3 \%$ & $\$ 127$ & $3 \%$ & $\$ 127$ & $3 \%$ \\
\hline Wash & $\$ 153$ & $4 \%$ & $\$ 153$ & $5 \%$ & $\$ 153$ & $4 \%$ & $\$ 153$ & $3 \%$ & $\$ 153$ & $4 \%$ \\
\hline Primary Treatment & $\$ 676$ & $18 \%$ & $\$ 550$ & $17 \%$ & $\$ 676$ & $17 \%$ & $\$ 676$ & $15 \%$ & $\$ 639$ & $17 \%$ \\
\hline Aqueous Waste & $\$ 150$ & $4 \%$ & $\$ 150$ & $5 \%$ & $\$ 150$ & $4 \%$ & $\$ 150$ & $3 \%$ & $\$ 150$ & $4 \%$ \\
\hline $\mathrm{APC}$ & $\$ 70$ & $2 \%$ & $\$ 70$ & $2 \%$ & $\$ 70$ & $2 \%$ & $\$ 70$ & $2 \%$ & $\$ 76$ & $2 \%$ \\
\hline Stabilization & $\$ 600$ & $16 \%$ & $\$ 525$ & $16 \%$ & $\$ 479$ & $12 \%$ & $\$ 515$ & $12 \%$ & $\$ 444$ & $12 \%$ \\
\hline Metal Recovery & $\$ 244$ & $6 \%$ & $\$ 244$ & $7 \%$ & $\$ 105$ & $3 \%$ & $\$ 105$ & $2 \%$ & $\$ 105$ & $3 \%$ \\
\hline Special Waste & $\$ 46$ & $1 \%$ & $\$ 46$ & $1 \%$ & $\$ 46$ & $1 \%$ & $\$ 46$ & $1 \%$ & $\$ 46$ & $1 \%$ \\
\hline Certify \& Ship & $\$ 346$ & $9 \%$ & $\$ 224$ & $7 \%$ & $\$ 424$ & $12 \%$ & $\$ 758$ & $17 \%$ & $\$ 424$ & $11 \%$ \\
\hline Admin \& Support & $\$ 254$ & $7 \%$ & $\$ 254$ & $8 \%$ & $\$ 254$ & $7 \%$ & $\$ 254$ & $6 \%$ & $\$ 254$ & $7 \%$ \\
\hline Disposal & $\$ 484$ & $13 \%$ & $\$ 286$ & $9 \%$ & $\$ 688$ & $18 \%$ & $\$ 911$ & $21 \%$ & $\$ 707$ & $19 \%$ \\
\hline 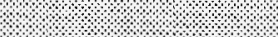 & & la & 6 & & & 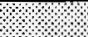 & 9 s/: & 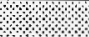 & & 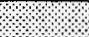 \\
\hline Total & $\$ 3,810$ & & $\$ 3,288$ & & $\$ 3,832$ & & $\$ 4,425$ & & $\$ 3,785$ & \\
\hline
\end{tabular}




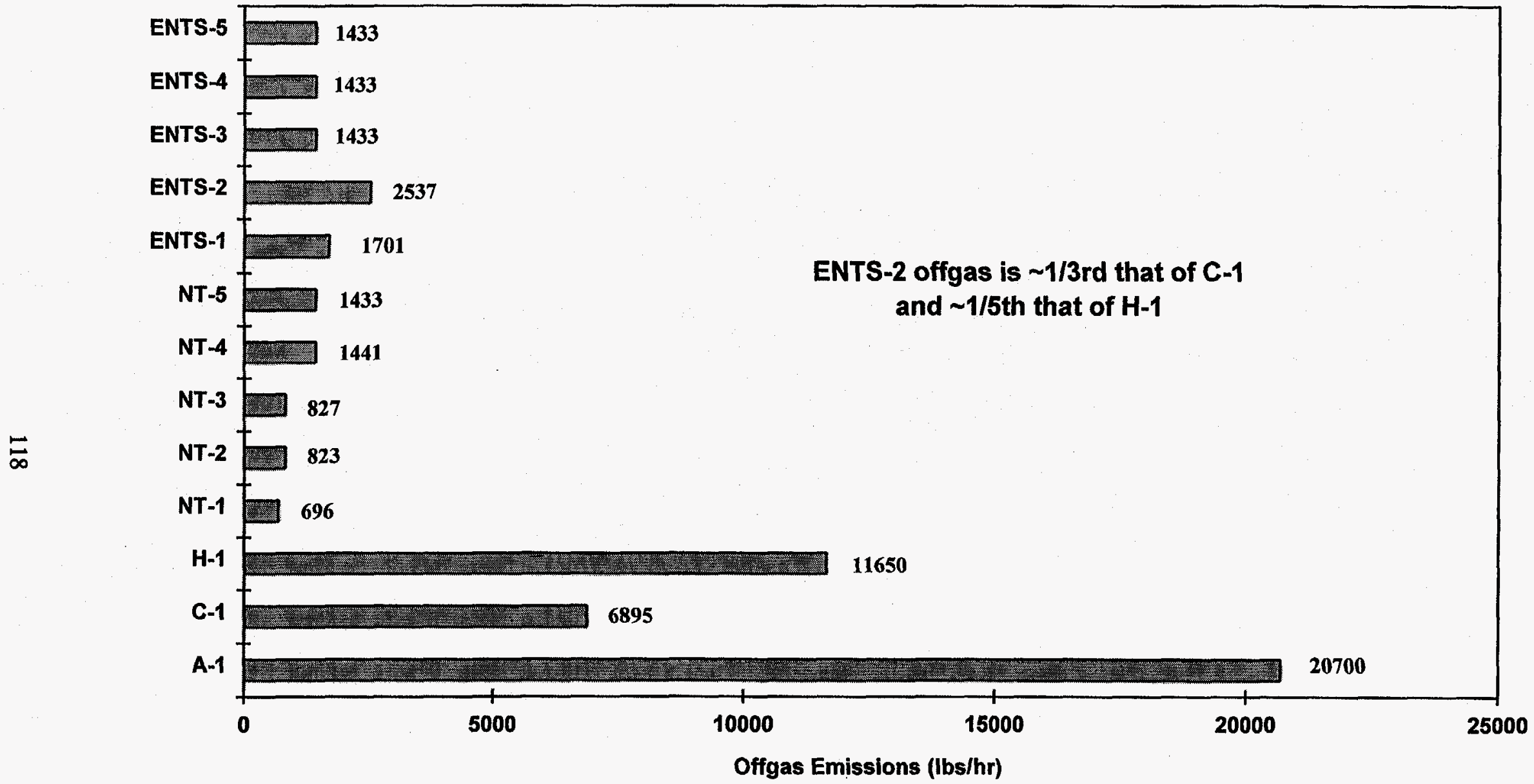

Figure 5-1 Offgas Emissions 


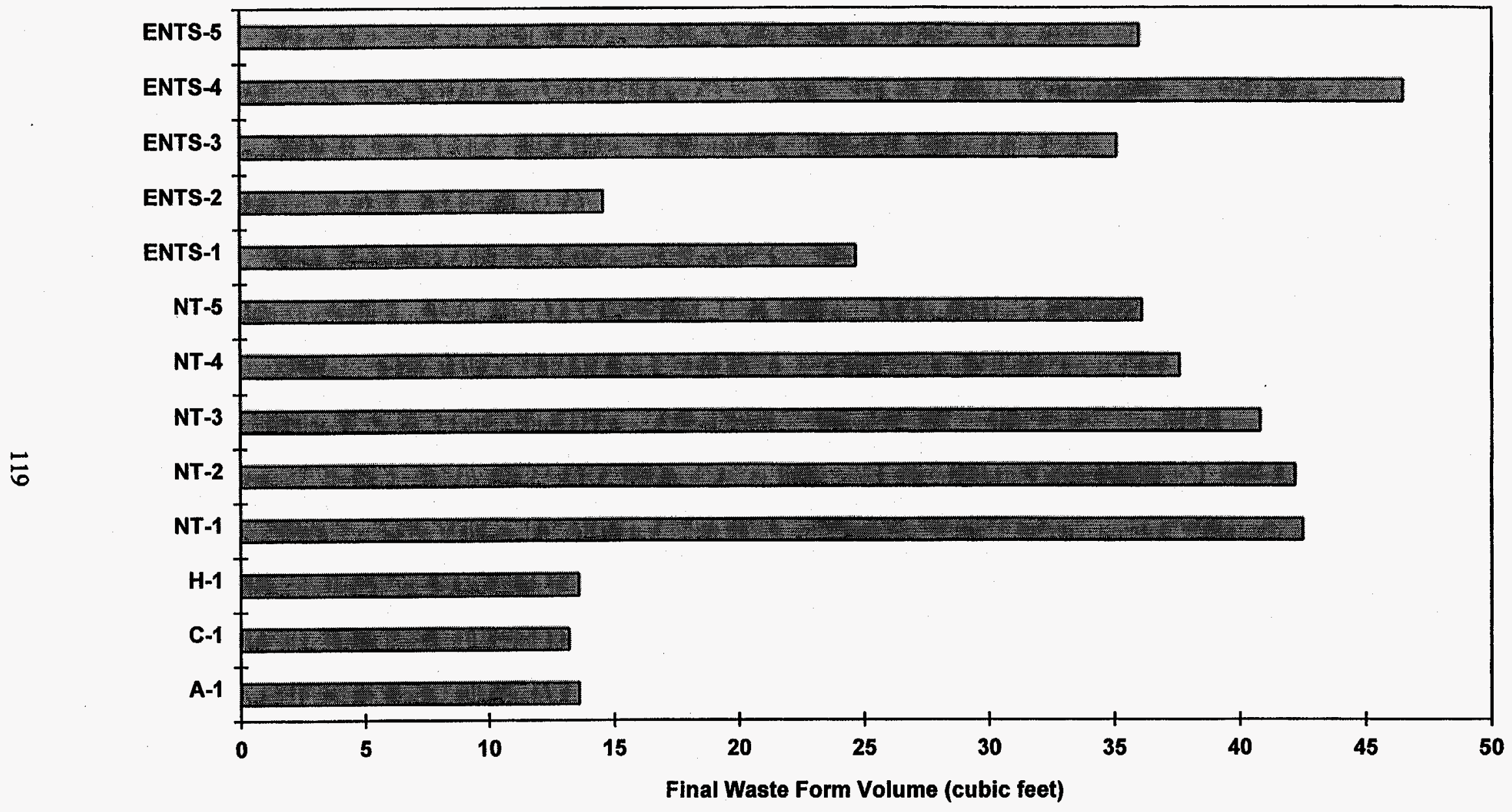

Figure 5-2 Final Waste Form Volume 


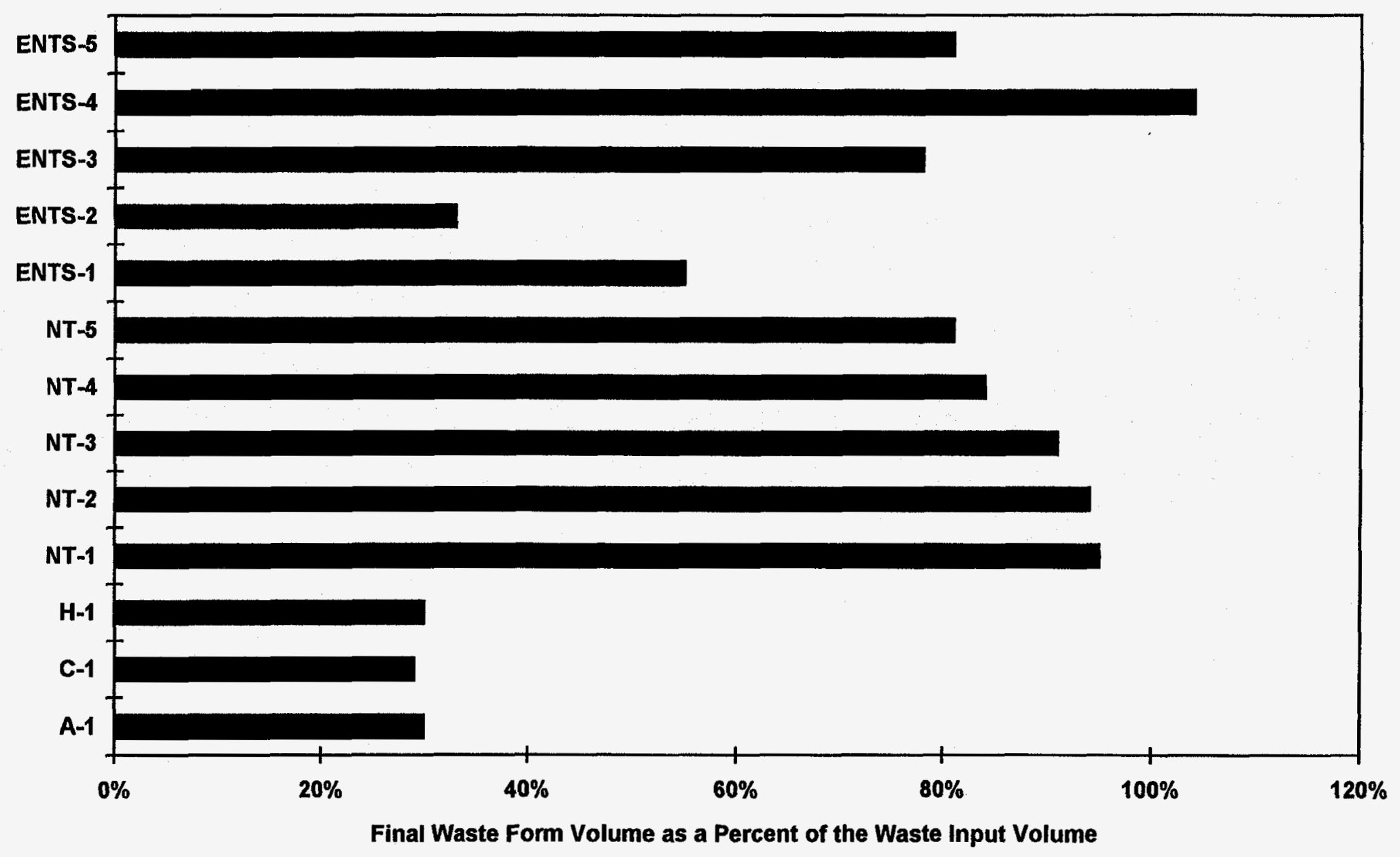

Figure 5-3 Volume Reduction 


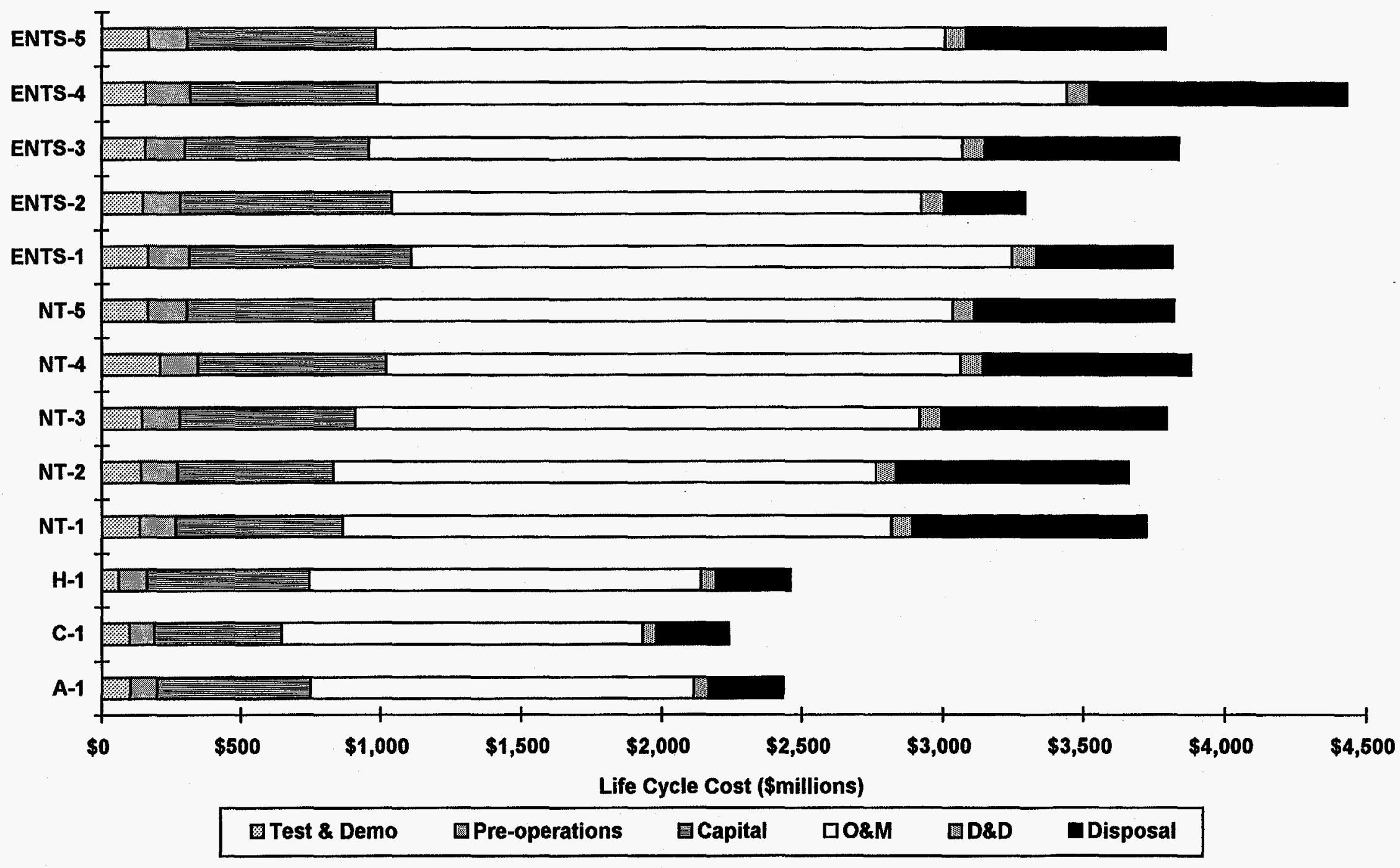

Figure 5-4 Total Life Cycle Cost 


\section{REFERENCES}

Aspen Technology, Inc, ASPEN PLUS, Version 9.13 for PCs, Cambridge, MA, 1994.

Biagi, Charles, and Julia Vetromile, Time and motion study jor Selected Systems jrom the Integrated Thermal and Nonthermal Treatment System Studies, prepared for Idaho National Engineering Laboratory, Idaho Falls, ID, February, 1997.

Biagi, Charles, William Quapp, Tom Bechtold, Daryoush Bahar, Blaine Brown, William Schwinkendorf, Ginger Swartz, Ben Teheranian, and Julia Vetromile, Integrated Nonthermal Treatment System Study, Final Drajt, prepared for Idaho National Engineering Laboratory, Idaho Falls, ID, January, 1997.

Dhooge, Patrick M., Delphi Corporation, letter of May 3, 1996 to Julia Vetromile of Morrison Knudsen Corp.

Dosani, M., IT Corporation, personal communication to Kathy Nakazawa of Morrison Knudsen Corporation, August 8, 1995, San Francisco, CA.

Dosani, M.A., M.L. Taylor, and N.P. Barkley, "Field Experience with a Full-Scale Debris-Washing Process", paper presented at the 87th Annual Air and Water Management Association Meeting, June 24, 1994.

Feizollahi, Fred and William J. Quapp, Integrated Thermal Treatment System Study -Phase 2 Results, EGG-MS-11211, prepared for Idaho National Engineering Laboratory, Idaho Falls, ID, August, 1995.

Peters, Max, and Klaus Timmerhaus, Plant Design and Economics jor Chemical Engineers, Second Edition, McGraw Hill Book Company, San Francisco, 1968.

Shaw, Richard, Wrap $2 A$ Process Control and Data Acquisition Design Basis, Waste Receiving and Processing Facility, by Raytheon Engineers for the U.S. Department of Energy, Richland, WA, January 1995

Taylor, M.L., M.A. Dosani, and N.P. Barkley, "Field Experience With a Full-Scale Debris Washing Process", paper presented at the 87th AWMA Meeting, June 24, 1994.

U. S. Code of Federal Regulations, 40 CFR 268.45, Treatment standards for hazardous debris.

U.S. Environmental Protection Agency, Technology Evaluation Report: Design and Development oj a PilotScale Debris Decontamination System, Volume 1, EPA/540/5-91/006a, August, 1991. 


\section{APPENDIX A}

\section{Figures}

Presented under a separate cover 

APPENDIX B

Cost Data 



\section{B-1 ENTS-1 Cost Tables}

B-1.1. Equipment Purchase and Installation Budget Estimates

B-1.2. Development, Testing and Evaluation Cost Estimate

B-1.3. Building, Equipment and Installation Cost Summary

B-1.4. Annual Operating and Maintenance Costs

B-1.5. Decontamination and Decommissioning Costs

B-1.6 Total Life-Cycle Cost (without Disposal) Estimate Summary for Enhanced Integrated Nonthermal Treatment Systems 

Table B-1.1: Equipment Purchase \& Installation Budget Estimates

Enhanced Non-Thermal Treatment System

ENTS - 1 CWO, Desorption and Vitrification

\begin{tabular}{|c|c|c|c|c|c|c|c|c|c|}
\hline \multirow{3}{*}{\multicolumn{2}{|c|}{ DESCRIPTION }} & \multicolumn{8}{|c|}{$\begin{array}{c}\text { ENTS - } 1 \\
\text { CWO, Desorption and Vitrification Case }\end{array}$} \\
\hline & & \multirow{2}{*}{$\begin{array}{l}\text { FAC } \\
\text { CAT }\end{array}$} & \multirow{2}{*}{ HP } & \multicolumn{3}{|c|}{ MATLS. \& EQUIP. } & \multicolumn{3}{|c|}{ INST. COSTS } \\
\hline & & & & QTY & \begin{tabular}{|c} 
Unit Cost \\
S1000's
\end{tabular} & $\begin{array}{l}\text { Amount } \\
\text { \$1000's }\end{array}$ & $\begin{array}{c}\text { Unit Cost } \\
\text { S1000's }\end{array}$ & $\begin{array}{l}\text { Amount } \\
\text { S1000's }\end{array}$ & $\begin{array}{l}\text { Total } \\
\text { U.O. }\end{array}$ \\
\hline & Adninistration Building Subsvoten & 巸 & & & & & & 78.78 & 17.7. \\
\hline & - Administration Office & & & & & & & & \\
\hline & - Office Furniture - EMPLOYEE & E & & 49 & 7.0 & 343 & 1.0 & 49 & 392 \\
\hline & - Computers & E & & Lot & 500.0 & 500 & 50.0 & 50 & 550 \\
\hline & - Communication & E & & Lot & 250.0 & 250 & 35.0 & 35 & 285 \\
\hline & - Testing Laboratory & & & & & & & & \\
\hline & - ICP-Mass Spectrometer & $\bar{D}$ & & 1 & & & & & \\
\hline & - Gas Chromatography - Mass Spec. & D & & 1 & & & & & \\
\hline & - Scintillation Counter - Alpha & D & & 1 & & & & & \\
\hline & - Scintillation Counter - Gamma & D & & 1 & & & & & \\
\hline & - Geiger - Muller Counter & D & & 1 & & & & & \\
\hline & - Fume Hood & A & & 2 & & & & & \\
\hline & - Centrifuge & $A$ & & 2 & & & & & \\
\hline & - Autoclave & $\mathrm{A}$ & & 1 & & & & & \\
\hline & - Scrubber/Blower & C & & 1 & & & & & \\
\hline & - Hood/HEPA Filtration & C & & 1 & & & & & \\
\hline & - Glovebox & D & & 1 & & & & & \\
\hline & - Wet Chemistry Equipment & $\mathrm{D}$ & & 1 & & & & & \\
\hline & - Refrigeration / Storage & $A$ & & 1 & & & & & \\
\hline & - Computer \& Data Communication & $D$ & & 2 & & & & & \\
\hline & - Atomic Absorption Spectrography & D & & 1 & & & & & \\
\hline & - Soil / Physical Testing Equipment & D & & Lot & & & & & \\
\hline & - Complete Package per Above Detail & $\bar{E}$ & & Lot & $1,500.0$ & 1500 & 300.0 & 300 & 1800 \\
\hline & - Office Equipment \& Furnishing & $\mathrm{E}$ & & Lot & 80.0 & 80 & 7.0 & 7 & 87 \\
\hline & - Allowance for Mechanical/Piping & E & & Lot & & 26.7 & & 132.3 & 159 \\
\hline & - Allowance for Electrical/Control & $E$ & & Lot & & 401.0 & & 264.6 & 665.6 \\
\hline & - Calibration, Testing \& Startup & E & & Lot & & 62.0 & & 50.3 & 112.3 \\
\hline & - & & & & & & & & \\
\hline & Total Administration Building Subsystem & & & & & 3162.7 & & 888.2 & 4050.9 \\
\hline 2 & 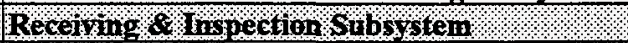 & 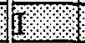 & ?. & 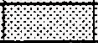 & 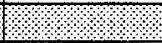 & 1/: & $6 / 19$. & 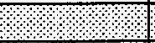 & \\
\hline & - Unloading, Staging \& Storage (A-RCINS) & & & & & & & & \\
\hline & - O/H Bridge Crane \& Rigging - 20 TN & E & & 1 & 625.0 & 625 & 125.0 & 125 & 750 \\
\hline & - Forklift - $10 \mathrm{TN}$ & $\mathrm{E}$ & & 2 & 65.0 & 130 & 10.0 & 20 & 150 \\
\hline & - Airlock & $\mathrm{E}$ & & 1 & 124.0 & 124 & 39.0 & 39 & 163 \\
\hline & - Inspect \& Assay (A-RCINS) & & & & & & & & \\
\hline & - Transfer Cart - 2 TN & B & & & & & & & \\
\hline & - Gamma Spectroscopy (GS) & D & & & & & & & \\
\hline & - Passive Active Neutron Assay (PAN) & $\mathrm{D}$ & & & & & & & \\
\hline & - Real Time Radiography (RTR) & D & & & & & & & \\
\hline & Load Cell & D & & & & & & & \\
\hline & - Closed Circuit TV & D & & & & & & & \\
\hline & - RHMMS Barrel Monitor including above & E & & 1 & $2,000.0$ & 2000 & 400.0 & 400 & 2400 \\
\hline & - Personnel Contamination Monitor & E & & 1 & 35.0 & 35 & 5.0 & 5 & 40 \\
\hline & - Container Decontamination Enclosure & $E$ & & 1 & 140.0 & 140 & 15.0 & 15 & 155 \\
\hline & - Open, Dump, \& Sort & & & & & & & & \\
\hline & - Self Guided Vehicle & E & & 2 & 425.0 & 850 & 64.0 & 128 & 978 \\
\hline & - Decap Device & E & & 3 & 50.0 & 150 & 9.0 & 27 & 177 \\
\hline & - Sort Hopper & E & & 5 & 40.0 & 200 & 4.0 & 20 & 220 \\
\hline & - Sort/Rotating Table & E & & 5 & 200.0 & 1000 & 30.0 & 150 & 1150 \\
\hline & - Special Waste Glove Box & $E$ & & 1 & 400.0 & 400 & 48.0 & 48 & 448 \\
\hline
\end{tabular}


Table B-1.1: Equipment Purchase \& Installation Budget Estimates

Enhanced Non-Thermal Treatment System

ENTS - 1 CWO, Desorption and Vitrification

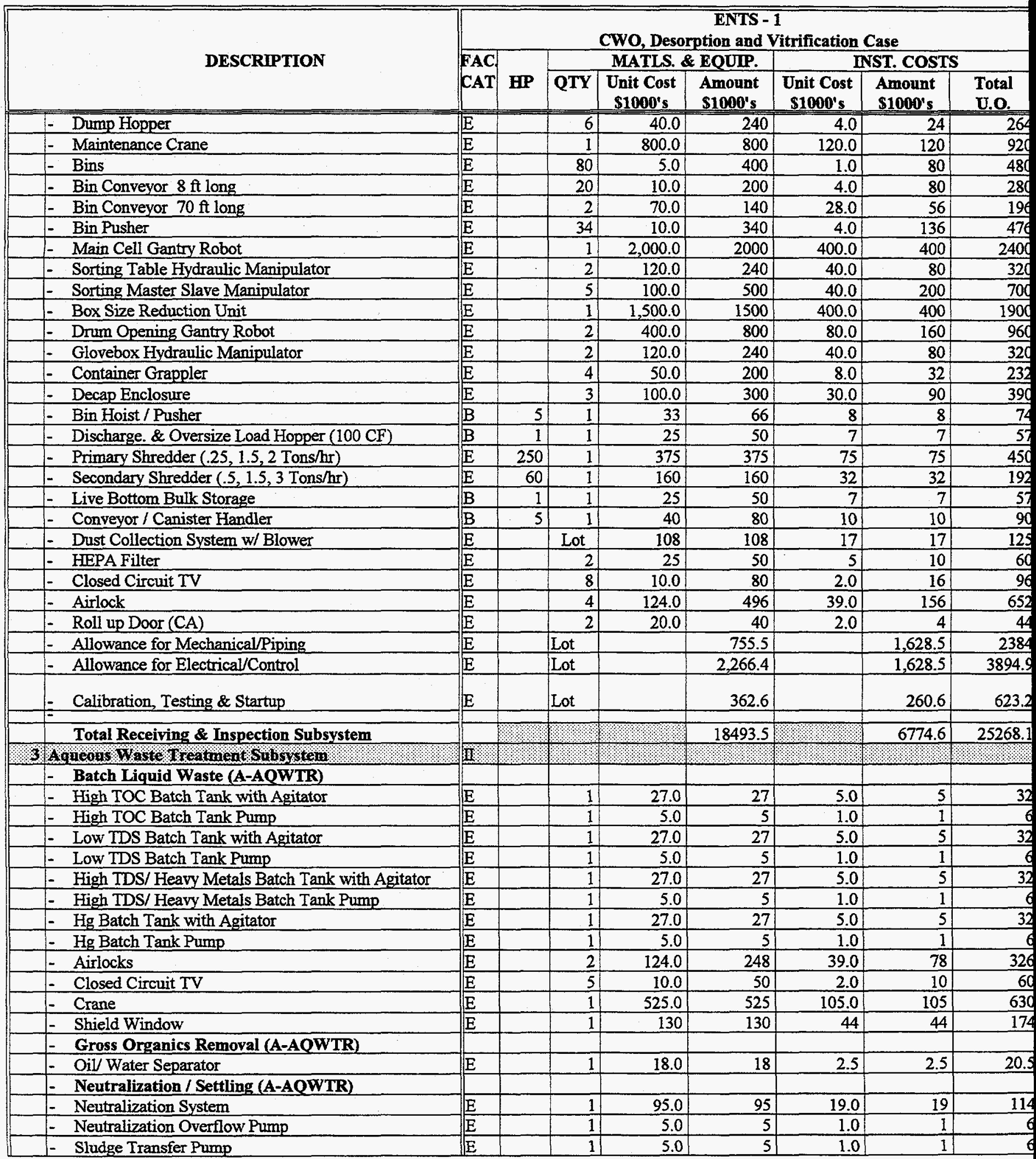


Table B-1.1: Equipment Purchase \& Installation Budget Estimates Enhanced Non-Thermal Treatment System ENTS - 1 CWO, Desorption and Vitrification

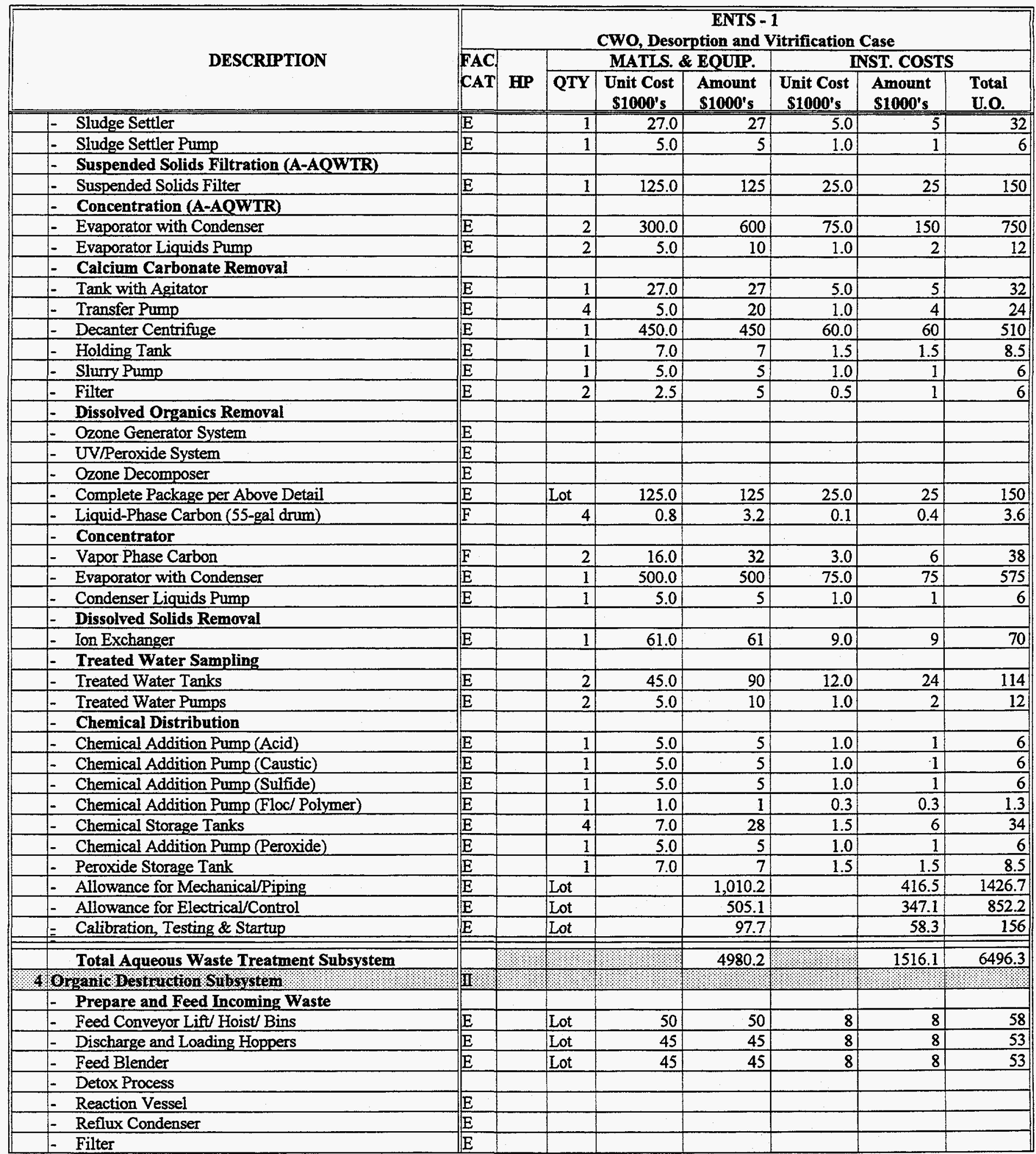


Table B-1.1: Equipment Purchase \& Installation Budget Estimates

Enhanced Non-Thermal Treatment System

ENTS - 1 CWO, Desorption and Vitrification

\begin{tabular}{|c|c|c|c|c|c|c|c|c|}
\hline \multirow{3}{*}{ DESCRIPTION } & \multicolumn{8}{|c|}{$\begin{array}{c}\text { ENTS - 1 } \\
\text { CWO, Desorption and Vitrification Case }\end{array}$} \\
\hline & \multirow{2}{*}{$\begin{array}{l}\text { FAC } \\
\text { CAT }\end{array}$} & \multirow{2}{*}{ HP } & \multicolumn{3}{|c|}{ MATLS. \& EQUIP. } & \multicolumn{3}{|c|}{ INST. COSTS } \\
\hline & & & QTY & $\begin{array}{c}\text { Unit Cost } \\
\text { \$1000's }\end{array}$ & $\begin{array}{l}\text { Amount } \\
\text { \$1000's }\end{array}$ & $\begin{array}{c}\text { Unit Cost } \\
\text { S1000's }\end{array}$ & $\begin{array}{l}\text { Amount } \\
\text { S1000's }\end{array}$ & $\begin{array}{l}\text { Total } \\
\text { U.O. }\end{array}$ \\
\hline - Solids Feeder & $E$ & & & & & & & \\
\hline - Solution Cooler & E & & & & & & & \\
\hline - Overhead Condenser & $E$ & & & & & & & \\
\hline - Pumps & E & & & & & & & \\
\hline - Condensate Catch & E & & & & & & & \\
\hline - Neutralization Tanks & E & & & & & & & \\
\hline - Filtered Solids Rinse Tanks & E & & & & & & & \\
\hline - Oil Heating and Cooling System & E & & & & & & & \\
\hline - Total Cost For Above Package & E & & Lot & 2400 & 2400 & 300 & 300 & 2700 \\
\hline Miscellaneous & & & & & & & & \\
\hline - Neutralization System & $\mathrm{E}$ & & 1 & 95 & 95 & 19 & 19 & 11 \\
\hline - Neutralization Overflow Pump & $E$ & & 1 & 5 & 5 & 1 & 1 & \\
\hline - Sludge Transfer Pump & E & & 1 & 5 & 5 & 1 & 1 & \\
\hline - Crane & $E$ & & 1 & 525 & 525 & 105 & 105 & 630 \\
\hline - Closed Circuit TV & $E$ & & 8 & 10 & 80 & 2 & 16 & 9 \\
\hline - Airlock & $E$ & & 2 & 124 & 248 & 39 & 78 & 32 \\
\hline - Shield Window & $E$ & & 3 & 130 & 390 & 44 & 132 & 522 \\
\hline - Allowance for Mechanical/Piping & $\mathrm{E}$ & & Lot & & $1,166.4$ & & 405.6 & 1572 \\
\hline - Allowance for Electrical/Control & $E$ & & Lot & & 583.2 & & 338.0 & 921.2 \\
\hline$=$ Calibration, Testing \& Startup & $E$ & & Lot & & 112.8 & & 56.8 & 169. \\
\hline Total Organic Destruction Subsystem & 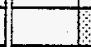 & & & & 5750.4 & & 1476.4 & 7226.8 \\
\hline S Residue \& Sludge Treaturent Subsystem. & in: & & & \% & & \% & & \\
\hline - Feed Preparation and Size Reduction & & & & & & & & \\
\hline - Feed Conveyor Lift/Hoist/Bins & $\mathrm{E}$ & & 1 & 50 & 50 & 8 & 8 & 58 \\
\hline - Discharge. \& Oversize Load Hopper (100 CF) & $\mathbf{E}$ & & 1 & 45 & 45 & 8 & 8 & 5 \\
\hline - Manipulate Crane $10 \mathrm{TN}$ & $\mathrm{E}$ & 15 & 1 & 525 & 525 & 105 & 105 & 63 \\
\hline - Shield Window & $\mathrm{E}$ & & 1 & 130.0 & 130 & 44.0 & 44 & 17 \\
\hline - Airlock & $\bar{E}$ & & 2 & 124 & 248 & 39 & 78 & $\overline{32}$ \\
\hline - Closed Circuit TV & $\mathrm{E}$ & & 10 & 10 & 100 & 2 & 20 & 12 \\
\hline - Feed Blender & $\bar{E}$ & & 1 & 45 & 45 & 8 & 8 & \\
\hline - Dewatering System & $\mathrm{E}$ & & 1 & & & & & \\
\hline - THERMAL DESORPTION & & & & & & & & \\
\hline - High Vacuum Dryer & & & 1. & & & & & \\
\hline - Nitrogen gas storage and feed tank & E & & 1 & & & & & \\
\hline - Vacuum Pump & $E$ & & 1 & & & & & \\
\hline Filter & $\mathrm{E}$ & & 1 & & & & & \\
\hline - Hot Oil System & & & & & & & & \\
\hline - Oil Storage and Feed Tank & E & & 1 & & & & & \\
\hline - Oil Heater & $\mathrm{E}$ & & 1 & & & & & \\
\hline - Fuel Storage and Feed Tank & $\mathrm{E}$ & & 1 & & & & & \\
\hline - Condensers & & & & & & & & \\
\hline - HEPA Filter for particulates & $\mathrm{E}$ & & 1 & & & & & \\
\hline - Induced Air Fan & $E$ & & 1 & & & & & \\
\hline - Primary Condensers (Air Cooler) & $\mathrm{E}$ & & 1 & & & & & \\
\hline - Secondary Condensers (Water Chiller) & $\mathrm{E}$ & & 2 & & & & & \\
\hline - Total per Package Above & $E$ & & Lot & 1700 & 1700 & 170 & 170 & 187 \\
\hline - Mercury Removal System & & & & & & & & \\
\hline - Mercury Precipitation Tank & $E$ & & 1 & 95 & 95 & 19 & 19 & \\
\hline - Iodine Recovery Tank & $E$ & & 1 & 27 & 27 & 5 & 5 & \\
\hline
\end{tabular}


Table B-1.1: Equipment Purchase \& Installation Budget Estimates Enhanced Non-Thermal Treatment System

ENTS - 1 CWO, Desorption and Vitrification

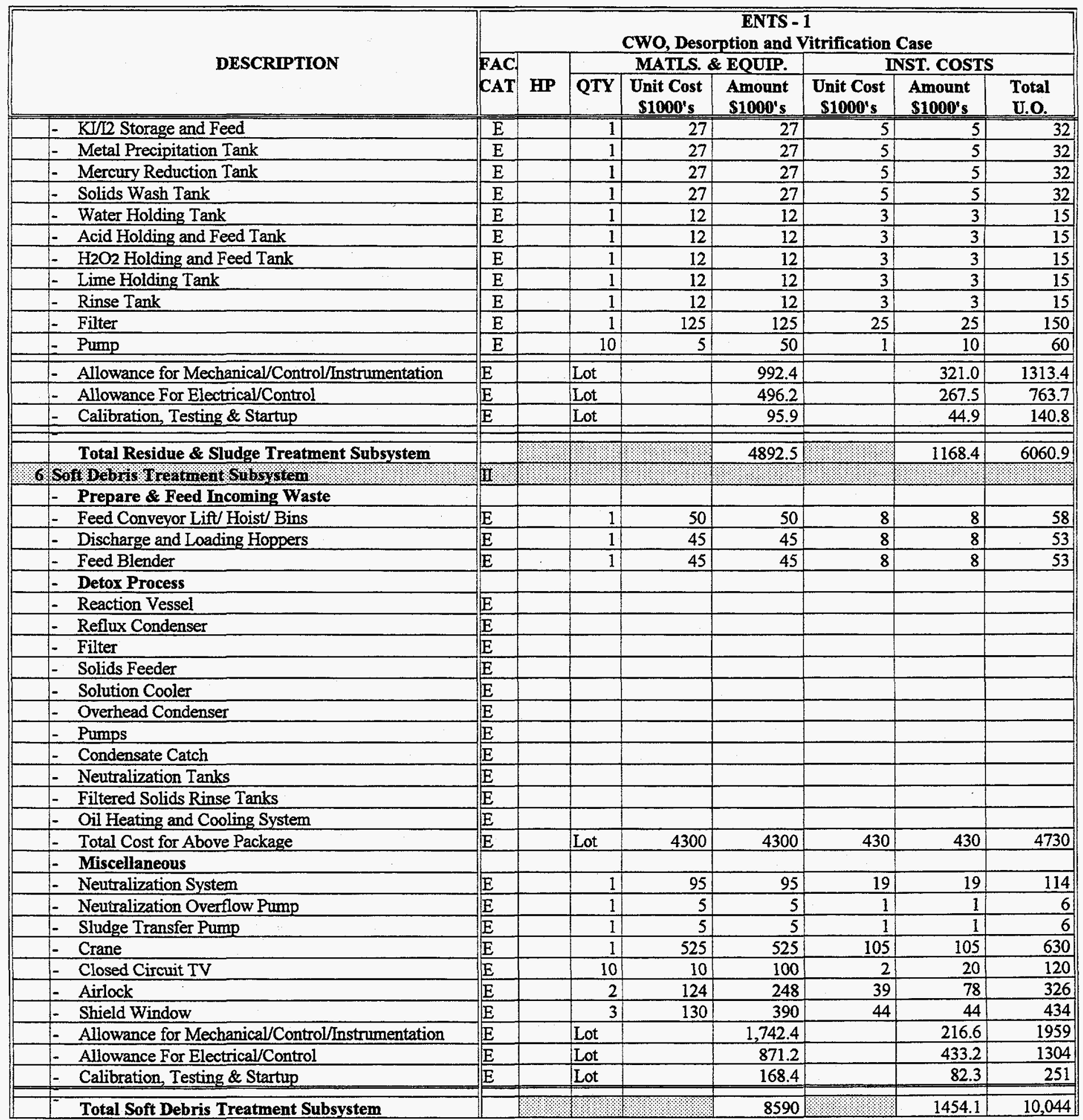


Table B-1.1: Equipment Purchase \& Installation Budget Estimates

Enhanced Non-Thermal Treatment System

ENTS - 1 CWO, Desorption and Vitrification

\begin{tabular}{|c|c|c|c|c|c|c|c|c|}
\hline \multirow{3}{*}{ DESCRIPTION } & \multicolumn{8}{|c|}{$\begin{array}{c}\text { ENTS - 1 } \\
\text { CWO, Desorption and Vitrification Case }\end{array}$} \\
\hline & \multirow{2}{*}{$\begin{array}{l}\text { FAC } \\
\text { CAT }\end{array}$} & \multirow{2}{*}{ HP } & \multicolumn{3}{|c|}{ MATLS. \& EQUTP. } & \multicolumn{3}{|c|}{ INST. COSTS } \\
\hline & & & QTY & \begin{tabular}{|c|} 
Unit Cost \\
S1000's
\end{tabular} & $\begin{array}{l}\text { Amount } \\
\text { \$1000's }\end{array}$ & $\begin{array}{c}\text { Unit Cost } \\
\text { S1000's }\end{array}$ & $\begin{array}{l}\text { Amount } \\
\text { \$1000's }\end{array}$ & $\begin{array}{l}\text { Total } \\
\text { U.O. }\end{array}$ \\
\hline \multicolumn{9}{|l|}{7 Complex Debris Treatment Subsystem } \\
\hline - Costs included with Grout & & & & & & & & \\
\hline \multicolumn{9}{|l|}{ - Total Complex Debris Treatment Subsystem } \\
\hline 8 Open Debris Treatment Subsystem $/ 1,1$, & II: & & & \% & 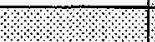 & 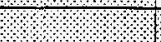 & 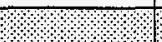 & \\
\hline - Feed Preparation and Separation & & & & & & & & \\
\hline - Bulk Solids Dump Hopper & F & & 1 & 40 & 40 & 4 & 4 & 44 \\
\hline - Bin Hoist/Pusher & $E$ & & 2 & 33 & 66 & 8 & 16 & 82 \\
\hline - Drum/Bin Conveyor & E & & 6 & 10 & 60 & 4 & 24 & 84 \\
\hline - OH Crane & E & & 1 & 525 & 525 & 105 & 105 & 630 \\
\hline I- Closed Circuit TV & E & & 7 & 10 & 70 & 2 & 14 & 84 \\
\hline - Airlock & $\mathrm{E}$ & & 2 & 124 & 248 & 39 & 78 & 326 \\
\hline - Shield Window & $E$ & & 1 & 130 & 130 & 44 & 44 & 174 \\
\hline - Dust Collection Assembly & $E$ & & Lot & 108 & 108 & 17 & 17 & 125 \\
\hline - High Pressure Wash & & & & & & & & \\
\hline - Receptacle Basket & & & & & & & & \\
\hline Water Pump & & & & & & & & \\
\hline - Agitation Pump & & & & & & & & \\
\hline - Surfactant Preparation & & & & & & & & \\
\hline - Surfactant Pump & & & & & & & & \\
\hline - Surfactant Holding Tank & & & & & & & & \\
\hline - Mixing Tank with Agitator & & & & & & & & \\
\hline - Solution High Pressure Pump & & & & & & & & \\
\hline - Water Heater & & & & & & & & \\
\hline - Water Holding Tank & & & & & & & & \\
\hline - Water Pump & & & & & & & & \\
\hline - Gross Organic Removal & & & & & & & & \\
\hline - Oil/Water Separator & & & & & & & & \\
\hline - Recycle Pump & & & & & & & & \\
\hline - Oil Pump & & & & & & & & \\
\hline - Water Treatment & & & & & & & & \\
\hline - Bag Filter & & & & & & & & \\
\hline Solids Bin & & & & & & & & \\
\hline - Neutralization Tank & & & & & & & & \\
\hline - Chemical Addition Pump (Acid) & & & & & & & & \\
\hline - Chemical Storage Tank & & & & & & & & \\
\hline - Liquid-Phase Carbon & & & & & & & & \\
\hline - Ion Exchange Unit & & & & & & & & \\
\hline Treated Water Holding Tank & & & & & & & & \\
\hline - Treated Water Pump & & & & & & & & \\
\hline - Total Per Wash Package Above & A & & Lot & 47 & 141 & 5 & 5 & 146 \\
\hline - Allowance for Mechanical/Control/Instrumentation & E & & Lot & & 416.4 & & 184.2 & 600.6 \\
\hline - Allowance For Electrical/Control & E & & Lot & & 208.2 & & 153.5 & 361.7 \\
\hline$\equiv$ Calibration, Testing \& Startup & $\underline{E}$ & & Lot & & 40.3 & & 25.8 & 66.1 \\
\hline Total Open Debris Treatment Subsystem & & & 3 & 8 & 2052.9 & & 670.5 & 2723.4 \\
\hline 9 Bull Soil Treatinent Subsystem $/ 7$ & II: & & & 3 & $1 \%$ & & 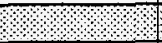 & 2.80 \\
\hline - Feed Preparation and Size Reduction & & & & & & & & \\
\hline - Feed Conveyor Lift/Hoist/Bins & E & & 1 & 30 & 30 & 6 & 6 & 36 \\
\hline - Discharge. \& Oversize Load Hopper & $\bar{E}$ & & 1 & 11 & 11 & 2 & 2 & 13 \\
\hline - Slurry Mix Tank & E & & 1 & 20 & 20 & 4 & 4 & 24 \\
\hline 1- Feed Pump & $\mathrm{E}$ & & 1 & 5 & 5 & 1 & 1 & 6 \\
\hline
\end{tabular}


Table B-1.1: Equipment Purchase \& Installation Budget Estimates Enhanced Non-Thermal Treatment System ENTS - 1 CWO, Desorption and Vitrification

\begin{tabular}{|c|c|c|c|c|c|c|c|c|}
\hline \multirow{3}{*}{ DESCRIPTION } & \multicolumn{8}{|c|}{$\begin{array}{c}\text { ENTS - } 1 \\
\text { CWO, Desorption and Vitrification Case }\end{array}$} \\
\hline & \multirow{2}{*}{$\begin{array}{l}\text { FAC } \\
\text { CAT }\end{array}$} & \multirow{2}{*}{ HP } & \multirow[b]{2}{*}{ QTY } & \multicolumn{2}{|c|}{ MATLS. \& EQUIP. } & \multicolumn{3}{|c|}{ INST. COSTS } \\
\hline & & & & \begin{tabular}{|c|} 
Unit Cost \\
S1000's
\end{tabular} & $\begin{array}{l}\text { Amount } \\
\text { S1000's }\end{array}$ & $\begin{array}{c}\text { Unit Cost } \\
\$ 1000^{\prime} s\end{array}$ & $\begin{array}{l}\text { Amount } \\
\text { S1000's }\end{array}$ & $\begin{array}{l}\text { Total } \\
\text { U.O. }\end{array}$ \\
\hline - Water Pump & E & & 1 & 5 & 5 & 1 & 1 & 6 \\
\hline - Airlock & E & & 2 & 124 & 248 & 39 & 78 & 326 \\
\hline - Closed Circuit TV & $E$ & & 7 & 10 & 70 & 2 & 14 & 84 \\
\hline - Shield Window & $E$ & & 1 & 130 & 130 & 44 & 44 & 174 \\
\hline - Wash System & & & & & & & & \\
\hline Attrition Mill & $\mathrm{D}$ & & 1 & 25 & 37.5 & 5 & 5 & 42.5 \\
\hline - Extraction Tank with Agitator & $\mathrm{D}$ & & 1 & 50 & 74.4 & 10 & 9.9 & 84.3 \\
\hline - Water Pump & E & & 1 & 5 & 5 & 1 & 1 & 6 \\
\hline - Slurry Pump & E & & 1 & 5 & 5 & 1 & 1 & 6 \\
\hline - Off-Gas Control & & & & & & & & \\
\hline - Heat Exchanger & E & & 2 & 6 & 12 & 0.4 & 0.8 & 12.8 \\
\hline - Solid/Liquid Separation System & $\mathrm{E}$ & & & & & & & \\
\hline - Vibrating Screen & E & & 1 & 6 & 6 & 1 & 1 & 7 \\
\hline - Hydrocyclone & D & & 2 & 4.5 & 13.5 & 0.4 & 0.8 & 14.3 \\
\hline - Countercurrent Solid/Liquid Contactor & B & & 1 & 160 & 320 & 32 & 32 & 352 \\
\hline - Water Pump & $E$ & & 1 & 5 & 5 & 1 & 1 & 6 \\
\hline - Slurry Pump & E & & 1 & 5 & 5 & 1 & 1 & 6 \\
\hline - Diaphragm Pump & $E$ & & 1 & 1 & 1 & 0.2 & 0.2 & 1.2 \\
\hline - Solids Bin & E & & 1 & 5 & 5 & 1 & 1 & 6 \\
\hline - Overhead Crane & E & & 1 & 525 & 525 & 105 & 105 & 630 \\
\hline - Solubility Reduction Separator & & & & & & & & \\
\hline - Phase Separator & $\mathrm{B}$ & & 1 & 4.8 & 9.6 & 1 & 1 & 10.6 \\
\hline - Additive Addition Pump & E & & 1 & 1 & 1 & 0.3 & 0.3 & 1.3 \\
\hline - Surfactant Phase Pump & E & & 1 & 5 & 5 & 1 & 1 & 6 \\
\hline - Water Phase Pump & E & & 1 & 5 & 5 & 1 & 1 & 6 \\
\hline - Storage Tanks (Water and Concentrate) & E & & 2 & 2 & 4 & 0.4 & 0.8 & 4.8 \\
\hline - Ultrafiltration Unit & $E$ & & 1 & 50 & 50 & 10 & 10 & 60 \\
\hline - Recycle Pump & $E$ & & 1 & 5 & 5 & 1 & 1 & 6 \\
\hline - Water Pump & E & & 1 & 5 & 5 & 1 & 1 & 6 \\
\hline - Dissolved Air Floatation Unit & $E$ & & 1 & 50 & 50 & 7.5 & 7.5 & 57.5 \\
\hline - $\quad$ Surfactant Preparation & & & & & & & & \\
\hline - Water Hold Tank & $E$ & & 1 & 2 & 2 & 0.2 & 0.2 & 2.2 \\
\hline - Water Heater & $E$ & & 1 & 0.6 & 0.6 & 0.1 & 0.1 & 0.7 \\
\hline - Surfactant Hold Tank & $E$ & & 1 & 2 & 2 & 0.2 & 0.2 & 2.2 \\
\hline - Water Pump & E & & 1 & 5 & 5 & 1 & 1 & 6 \\
\hline - $\quad$ Surfactant Pump & $E$ & & 1 & 5 & 5 & 1 & 1 & 6 \\
\hline - Dilution Tank Agitator & $\mathrm{E}$ & & 1 & 1.5 & 1.5 & 0.2 & 0.2 & 1.7 \\
\hline - Dilution Tank Pump & $\mathrm{E}$ & & 1 & 5 & 5 & 1 & 1 & 6 \\
\hline - Surfactant Dilution Tank & $E$ & & 1 & 2.0 & 2 & 0.4 & 0.4 & 2.4 \\
\hline - Dryer & $E$ & & 1 & 100.0 & 100 & 30.0 & 30 & 130 \\
\hline - $\quad$ Surfactant Separation & & & & & & & & \\
\hline - Additive Pump & $E$ & & 4 & 1 & 4 & 0.3 & 1.2 & 5.2 \\
\hline - Additive Tanks & $E$ & & 1 & 3.2 & 3.2 & 0.5 & 0.5 & 3.7 \\
\hline - Surfactant Separation Unit & $\mathrm{B}$ & & 1 & 50 & 100 & 10 & 10 & 110 \\
\hline - Recycle Pump & $\mathrm{E}$ & & 1 & 5 & 5 & 1 & 1 & 6 \\
\hline - Water Pump & $\mathrm{E}$ & & 1 & 5 & 5 & 1 & 1 & 6 \\
\hline - Allowance for Mechanical/Control/Instrumentation & $\mathrm{E}$ & & Lot & & 572.5 & & 228.7 & 801.2 \\
\hline - Allowance For Electrical/Control & E & & Lot & & 286.2 & & 190.6 & 476.8 \\
\hline - Calibration, Testing \& Startup & $E$ & & Lot & & 55.3 & & 32.0 & 87.3 \\
\hline Total Bulk Soil Treatment Subsystem & & & & 6 & 2822.3 & & 832.4 & 3654.7 \\
\hline
\end{tabular}


Table B-1.1: Equipment Purchase \& Installation Budget Estimates

Enhanced Non-Thermal Treatment System

ENTS - 1 CWO, Desorption and Vitrification

\begin{tabular}{|c|c|c|c|c|c|c|c|c|c|}
\hline \multirow{3}{*}{\multicolumn{2}{|c|}{ DESCRIPTION }} & \multicolumn{8}{|c|}{$\begin{array}{c}\text { ENTS - 1 } \\
\text { CWO, Desorption and Vitrification Case }\end{array}$} \\
\hline & & \multirow{2}{*}{$\begin{array}{l}\text { FAC } \\
\text { CAT }\end{array}$} & \multirow[b]{2}{*}{$\mathbf{H P}$} & \multicolumn{3}{|c|}{ MATLS. \& EQUIP. } & \multicolumn{3}{|c|}{$\begin{array}{l}\text { INST. COSTS } \\
\end{array}$} \\
\hline & & & & QTY & $\begin{array}{l}\text { Unit Cost } \\
\text { S1000's }\end{array}$ & $\begin{array}{l}\text { Amount } \\
\text { S1000's }\end{array}$ & $\begin{array}{c}\text { Unit Cost } \\
\$ 1000 \text { 's }\end{array}$ & $\begin{array}{l}\text { Amount } \\
\text { S1000's }\end{array}$ & $\begin{array}{l}\text { Total } \\
\text { U.O. }\end{array}$ \\
\hline 10 & Meta Wecontamination Sübsystem & 1 & & $\sqrt{1}$ & & & & & \\
\hline & - Pretreatment (GTCC-II) & & & & & & & & \\
\hline & - High Pressure Water Rinse Station & & & & & & & & \\
\hline & - Hot Air Dryer Station & & & & & & & & \\
\hline & - Chemical Storage Tank & & & & & & & & \\
\hline & - Chemical Mixing Tank & & & & & & & & \\
\hline & - Chemical Metering Pump & & & & & & & & \\
\hline & - Solution Pump & & & & & & & & \\
\hline & - Complete Package per Above Detail & E & & Lot & 150.0 & 150 & 50.0 & 50 & 200 \\
\hline & - Shield Window & $\mathbf{E}$ & & 1 & 130 & 130 & 44 & 44 & 17 \\
\hline & - Closed Circuit TV & $\bar{E}$ & & 5 & 10.0 & 50 & 2.0 & 10 & \\
\hline & - $\quad$ Size Reduction (GTCC-II) & & & & & & & & \\
\hline & - Hydraulic Manipulator & $E$ & & 1 & 120.0 & 120 & 50.0 & 50 & 170 \\
\hline & - Gantry Robot & E & & 1 & 800.0 & 800 & 160.0 & 160 & 96 \\
\hline & Size Reduction Table and Tools & $\underline{E}$ & & 1 & 205.0 & 205 & 40.0 & 40 & 24 \\
\hline & - Fugitive Dust Collection System & $E$ & & 2 & 100.0 & 200 & 15.0 & 30 & 23 \\
\hline & - Master Slave Manipulator & $\mathrm{E}$ & & 1 & 65.0 & 65 & 15.0 & 15 & 81 \\
\hline & Airlock & E & & 2 & 124.0 & 248 & 39.0 & 78 & $\overline{32}$ \\
\hline & - Assay/Inspection (GTCC-I) & & & & & & & & \\
\hline & - Assay Unit - Mini & E & & 1 & 500.0 & 500 & 100.0 & 100 & 60 \\
\hline & - Roller Conveyor & E & & 10 & 6.0 & 60 & 1.0 & 10 & 7 \\
\hline & - Shielding Window & E & & 3 & 130.0 & 390 & 44.0 & 132 & 52 \\
\hline & - Allowance for Mechanical/Piping & $E$ & & Lot & & 145.9 & & 359.5 & 505. \\
\hline & - Allowance for Electrical/Control & $E$ & & Lot & & 437.7 & & 359.5 & 797. \\
\hline & - Calibration, Testing \& Startup & E & & Lot & & 70.0 & & 57.5 & 127. \\
\hline & & & & & & & & & \\
\hline & Total Metal Decontamination Subsystem & & & 3 & & 3571.6 & ' & 1495.5 & 5067. \\
\hline 11 & 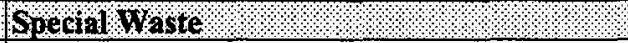 & II & & $1 \%$ & & 1 & 1.0 & ঝ & \\
\hline & - & & & & & & & & \\
\hline & - Allowance for Mechanical/Piping & E & & Lot & & & & & \\
\hline & - Allowance for Electrical/Control & $E$ & & Lot & & & & & \\
\hline & - Calibration, Testing \& Startup & $\mathrm{E}$ & & Lot & & & & & \\
\hline & & & & & & & & & \\
\hline & Total Special Waste & & 7 & 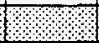 & 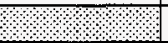 & & 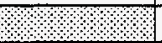 & & \\
\hline 12 & Mercury Amalgarnation Subsystem & Ii: & & 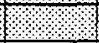 & 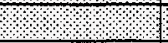 & 2 & 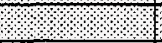 & 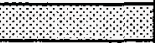 & \\
\hline & - Feed Storage \& Preparation (A-RMERC) & & & & & & & & \\
\hline & - Mercury Waste Feed Bin $\left(60 \mathrm{ft}^{3}\right)$ & F & & 1 & 20.0 & 20.0 & 4.0 & 4.0 & 24. \\
\hline & - Shredder/ Feeder & $F$ & & 1 & 90.0 & 90.0 & 15.0 & 15.0 & 105. \\
\hline & - Airlock & E & & 1 & 124.0 & 124.0 & 39.0 & 39.0 & 163. \\
\hline & - Crane & $\mathrm{F}$ & & 1 & 525.0 & 525.0 & 105.0 & 105.0 & 630. \\
\hline & - Closed Circuit TV & $E$ & & 2 & 10.0 & 20.0 & 10.0 & 20.0 & 40. \\
\hline & - Mercury Recovery (A-RMERC) & & & & & & & & \\
\hline & - Mercury Retort & F & & 1 & & & & & \\
\hline & - Mercury Condenser & E & & 1 & & & & & \\
\hline & - Mercury Separator & E & & 1 & & & & & \\
\hline & - Total Retort System & $E$ & & 1 & 300.0 & 300.0 & 80.0 & 80.0 & 380. \\
\hline & - Off-Gas Treatment (A-RMERC) & & & & & & & & \\
\hline & - Offgas Combustion Chamber & $E$ & & 1 & 130.0 & 130.0 & 10.0 & 10.0 & 140. \\
\hline & - Quencher & $\bar{E}$ & & 1 & 57.0 & 57.0 & 12.0 & 12.0 & 69. \\
\hline & - Dry Gas Filter & E & & 1 & 160.0 & 160.0 & 25.0 & 25.0 & 185. \\
\hline
\end{tabular}


Table B-1.1: Equipment Purchase \& Installation Budget Estimates

Enhanced Non-Thermal Treatment System

ENTS - 1 CWO, Desorption and Vitrification

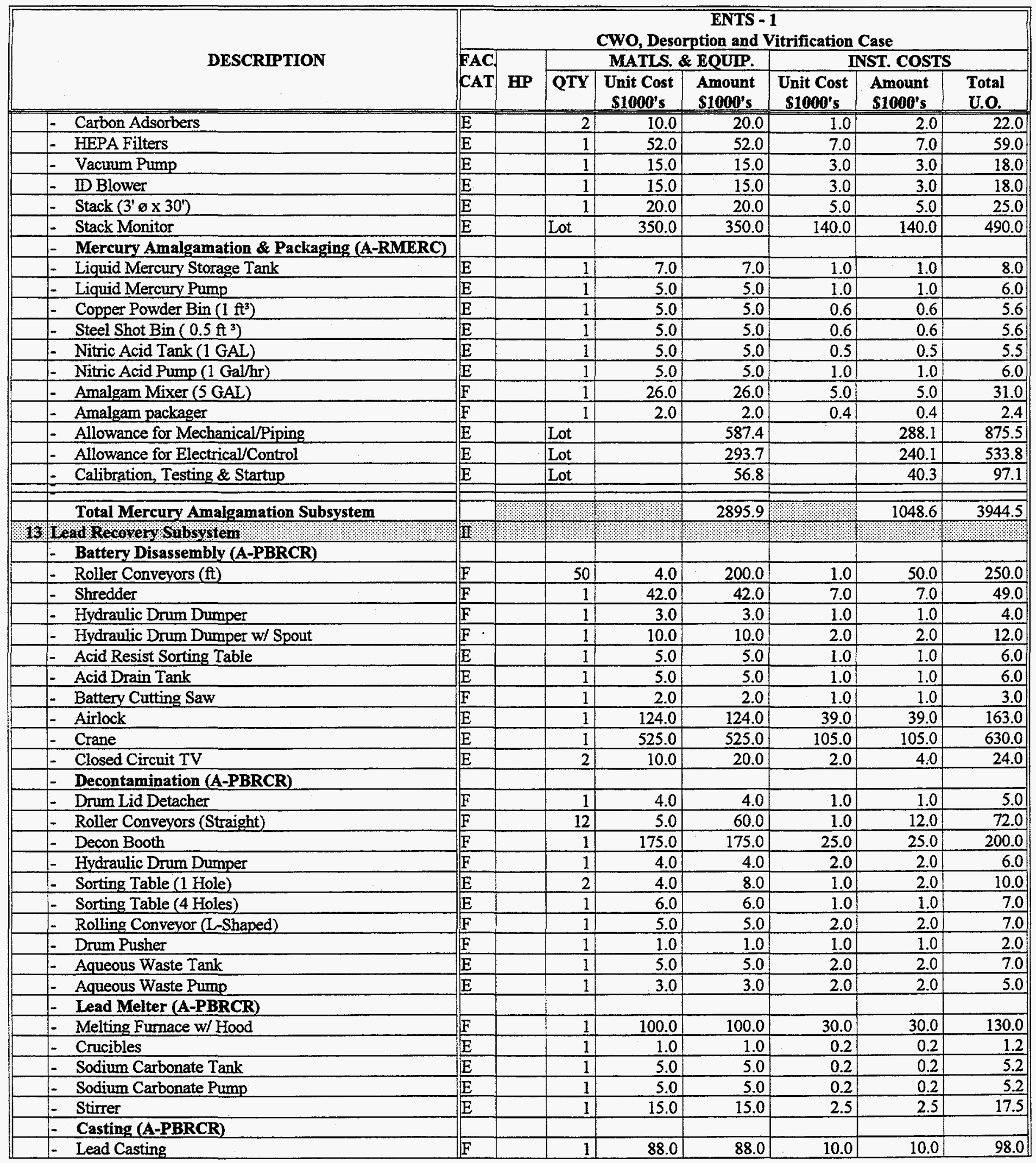


Table B-1.1: Equipment Purchase \& Installation Budget Estimates

Enhanced Non-Thermal Treatment System

ENTS - 1 CWO, Desorption and Vitrification

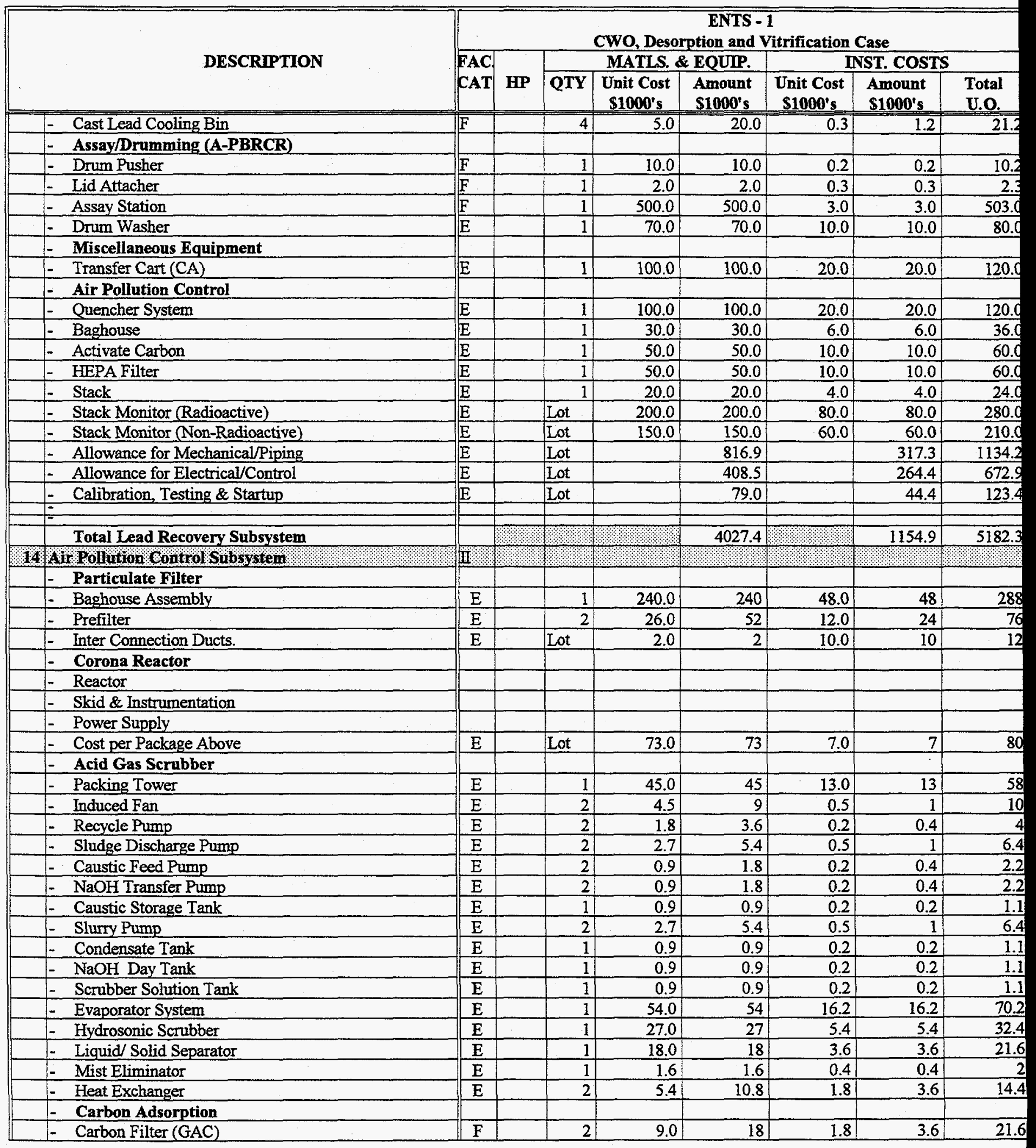


Table B-1.1: Equipment Purchase \& Installation Budget Estimates

Enhanced Non-Thermal Treatment System

ENTS - 1 CWO, Desorption and Vitrification

\begin{tabular}{|c|c|c|c|c|c|c|c|c|c|}
\hline \multirow{3}{*}{\multicolumn{2}{|c|}{ DESCRIPTION }} & \multicolumn{8}{|c|}{$\begin{array}{c}\text { ENTS - } 1 \\
\text { CWO, Desorption and Vitrification Case } \\
\end{array}$} \\
\hline & & \multirow{2}{*}{ FAC } & \multirow[b]{2}{*}{$\mathbf{H P}$} & \multicolumn{3}{|c|}{ MATLS. \& EQUIP. } & \multicolumn{3}{|c|}{ INST. COSTS } \\
\hline & & & & QTY & \begin{tabular}{|c|} 
Unit Cost \\
\$1000's \\
\end{tabular} & $\begin{array}{l}\text { Amount } \\
\text { \$1000's }\end{array}$ & $\begin{array}{l}\text { Unit Cost } \\
\text { S1000's }\end{array}$ & $\begin{array}{l}\text { Amount } \\
\text { S1000's }\end{array}$ & $\begin{array}{l}\text { Total } \\
\text { U.O. }\end{array}$ \\
\hline & - Carbon Filter (Sublimated GAC) & 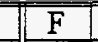 & & 2 & 9.0 & 18 & 1.8 & 3.6 & 21.6 \\
\hline & Support System & & & & & & & & \\
\hline & Stack & $E$ & & 1 & 7.2 & 7.2 & 1.4 & 1.4 & 8.6 \\
\hline & - Stack Monitors - Radioactive & $E$ & & Lot & 56.0 & 56 & 14.4 & 14.4 & 70.4 \\
\hline & - Stack Monitors - Non Radioactive & $E$ & & Lot & 27.0 & 27 & 10.8 & 10.8 & 37.8 \\
\hline & - OH Crane & $\mathrm{E}$ & & 1 & 525.0 & 525 & 105.0 & 105 & 630 \\
\hline & - Airlock & E & & 2 & 124.0 & 248 & 39.0 & 78 & 326 \\
\hline & - Closed Circuit TV & $E$ & & 4 & 10.0 & 40 & 2.0 & 8 & 48 \\
\hline & - Shield Window & $\bar{E}$ & & 1 & 130 & 130 & 44 & 44 & 174 \\
\hline & - Allowance for Mechanical/Piping & $\mathrm{E}$ & & Lot & & 487.0 & & 243.0 & 730 \\
\hline & - Allowance for Electrical/Control & $\mathrm{E}$ & & Lot & & 243.5 & & 202.5 & 446 \\
\hline & - Calibration, Testing \& Startup & E & & Lot & & 47.1 & & 34.0 & 81.1 \\
\hline & Total Air Pollution Control Subsystem & & & & & 2400.8 & & 884.5 & 3285.3 \\
\hline 15 & Polymer Stabilization Subsystem $/ .1 / 1$. & 1 & & 3 & (1., & 6.8 & 1 & 9.9 & \\
\hline & - Prepare \& Feed Incoming Waste (A-PLYMR) & & & & & & & & \\
\hline & - Bin Hoist / Pusher & $\mathrm{F}$ & 5 & 1 & 100.0 & 100 & 8.0 & 8 & 108 \\
\hline & Discharge. \& Oversize Load Hopper (100 CF) & F & 1 & 1 & 60.0 & 60 & 9.0 & 9 & 69 \\
\hline & - Tertiary Shredder & $\mathrm{B}$ & 50 & 1 & 50.0 & 100 & 10.0 & 10 & 110 \\
\hline & - Live Bottom Bulk Storage & F & 1 & 1 & 60.0 & 60 & 9.0 & 9 & 69 \\
\hline & - Conveyor / Canister Handler & F & 5 & 1 & 80.0 & 80 & 10.0 & 10. & 90 \\
\hline & - Manipulation Crane - 5 TON & $E$ & 10 & 1 & 525.0 & 525 & 105.0 & 105 & 630 \\
\hline & Dust Collection System w/ Blower & F & & 1 & 108.0 & 108 & 17.0 & 17 & 125 \\
\hline & - HEPA Filter & $F$ & & 8 & 25.0 & 200 & 5.0 & 40 & 240 \\
\hline & - Closed Circuit TV & $\mathrm{E}$ & & 13 & 10.0 & 130 & 2.0 & 26 & 156 \\
\hline & Airlock & $E$ & & 2 & 124.0 & 248 & 39.0 & 78 & 326 \\
\hline & Shield Window & E & & 2 & 130 & 260 & 44 & 88 & 348 \\
\hline & Drying (A-PLYMR) & & & & & & & & \\
\hline & Drier & $\mathrm{F}$ & & 1 & 585.0 & 585 & 88.0 & 88 & 673 \\
\hline & - Conveyor & F & 5 & 5 & 6.0 & 30 & 1.0 & 5 & 35 \\
\hline & - Proportioning and Blending (A-PLYMR) & & & & & & & & \\
\hline & - Polymer Pellets Storage Silo & E & & 4 & 25.0 & 100 & 7.0 & 28 & 128 \\
\hline & - Weigh Station & E & & 2 & 20.0 & 40 & 2.0 & 4 & 44 \\
\hline & Blender & $F$ & 2 & 1 & 53.0 & 53 & 5.0 & 5 & 58 \\
\hline & - Conveyor & $F$ & 5 & 11 & 6.0 & 66 & 1.0 & 11 & 77 \\
\hline & - Encapsulation (A-PLYMR) & & & & & & & & \\
\hline & Extruder & F & 100 & 1 & 630.0 & 630 & 22.7 & 22.7 & 652.7 \\
\hline & - Activated Carbon & F & & 4 & 10.0 & 40 & 1.0 & 4 & 44 \\
\hline & - Drum Cooling (A-PLYMR) & & & & & & & & \\
\hline & - Induced Draft Fan/ Ducting & F & & 8 & 16.0 & 128 & 2.0 & 16 & 144 \\
\hline & Enclosure & F & & 1 & 121.0 & 121 & 8.0 & 8 & 129 \\
\hline & - Drum Capping and Washing (A-PLYMR) & & & & & & & & \\
\hline & - Drum Wash/ Cap & $\mathrm{E}$ & & 4 & 70.0 & 280 & 15.0 & 60 & 340 \\
\hline & - Flush Water Collection and Conveying & E & & 2 & 30.0 & 60 & 18.0 & 36 & 96 \\
\hline & Conveyor & $\underline{F}$ & 5 & 39 & 6.0 & 234 & 1.0 & 39 & 273 \\
\hline & - Allowance for Mechanical/Piping & $E$ & & Lot & & 211.9 & & 363.4 & 575.3 \\
\hline & - Allowance for Electrical/Control & $E$ & & Lot & & 635.7 & & 363.4 & 999.1 \\
\hline & - Calibration, Testing \& Startup & $E$ & & Lot & & 101.7 & & 58.1 & 159.8 \\
\hline & Total Polymer Stabilization Subsystem & & & & & 5187.3 & & 1511.6 & 6698.9 \\
\hline
\end{tabular}


Table B-1.1: Equipment Purchase \& Installation Budget Estimates

Enhanced Non-Thermal Treatment System

ENTS - 1 CWO, Desorption and Vitrification

\begin{tabular}{|c|c|c|c|c|c|c|c|c|c|}
\hline \multirow{3}{*}{\multicolumn{2}{|c|}{ DESCRIPTION }} & \multicolumn{8}{|c|}{$\begin{array}{c}\text { ENTS - 1 } \\
\text { CWO, Desorption and Vitrification Case }\end{array}$} \\
\hline & & \multirow{2}{*}{ FAC } & \multirow{2}{*}{ HP } & \multirow[b]{2}{*}{ QTY } & \multicolumn{2}{|c|}{ MATLS. \& EQUIP. } & \multicolumn{3}{|c|}{$\begin{array}{r}\text { INST. COSTS } \\
\end{array}$} \\
\hline & & & & & $\begin{array}{c}\text { Unit Cost } \\
\$ 1000 ' s\end{array}$ & $\begin{array}{l}\text { Amount } \\
\text { \$1000's }\end{array}$ & $\begin{array}{c}\text { Unit Cost } \\
\text { S1000's }\end{array}$ & $\begin{array}{l}\text { Amount } \\
\text { S1000's }\end{array}$ & $\begin{array}{l}\text { Total } \\
\text { U.O. }\end{array}$ \\
\hline \multicolumn{10}{|c|}{16 Vitrification } \\
\hline & - Prepare and Feed Incoming Waste (A-VITRF) & & & & & & & & \\
\hline & - Bulk Solids Dump Hopper & $F$ & & 1 & 60.0 & 60.0 & 15.0 & 15.0 & 75.0 \\
\hline & - Shredding System & E & & 1 & $1,320.0$ & $1,320.0$ & 260.0 & 260.0 & $1,580.0$ \\
\hline & - Waste Prep Crane & F & & 1 & 525.0 & 525.0 & 105.0 & 105.0 & 630.0 \\
\hline & - Drum/Bin Conveyor & $E$ & & 20 & 6.0 & 120.0 & 1.0 & 20.0 & 140.0 \\
\hline & - Closed Circuit TV & $\mathrm{E}$ & & 2 & 10.0 & 20.0 & 2.0 & 4.0 & 24.0 \\
\hline & - Airlock & E & & 2 & 124.0 & 248.0 & 39.0 & 78.0 & 326.0 \\
\hline & Dust Collection Assembly & E & & 1 & 120.0 & 120.0 & 20.0 & 20.0 & 140.0 \\
\hline & - Melter (A-VITRF) & & & & & & & & \\
\hline & - Melter & & & 1 & & & & & \\
\hline & - Melter Feed Assembly & & & 1 & & & & & \\
\hline & - Melter Oxygen Feed Controller & & & 1 & & & & & \\
\hline & - Melter Inlet Air Blower & & & 1 & & & & & \\
\hline & - Melter Discharge System & & & 1 & & & & & \\
\hline & - Melter Room Temperature Monitoring \& Cooling & & & 1 & & & & & \\
\hline & - Melter Vacuum Pumps & & & 1 & & & & & \\
\hline & - Melter Slag/Ingot Vacuum Chamber & & & 1 & & & & & \\
\hline & - Melter Hearth Removal System & & & 2 & & & & & \\
\hline & - Cost Per Package Above & $F$ & & Lot & $4,000.0$ & $4,000.0$ & 800.0 & 800.0 & $4,800.0$ \\
\hline & - Maintenance Crane ( 5 TN) & E & & 1 & 525.0 & 525.0 & 105.0 & 105.0 & 630.0 \\
\hline & - Monorail Hoist $(5 \mathrm{TN})$ & F & & 1 & 85.0 & 85.0 & 20.0 & 20.0 & 105.0 \\
\hline & - Cool Melter (A-VITRF) & & & & & & & & \\
\hline & - Melter Cooling Water Unit & & & & & & & & \\
\hline & - Cooling Water Pump $150 \mathrm{Gal} / \mathrm{min}$ & & & & & & & & \\
\hline & - Cooling Water Pump $200 \mathrm{gal} / \mathrm{min}$ & & & & & & & & \\
\hline & - Cooling Water Pump 2500 GAL & & & & & & & & \\
\hline & - Elevated Water Tank & & & & & & & & \\
\hline & - Cooling Tower & & & & & & & & \\
\hline & - Circulation pump & & & & & & & & \\
\hline & - Complete Package per Above Detail & E & & Lot & 300.0 & 300.0 & 70.0 & 70.0 & 370.0 \\
\hline & - Prepare and Feed Soil (A-VITRF) & & & & & & & & \\
\hline & - Melter Soil Feeder & $\mathrm{E}$ & & 1 & 150.0 & 150.0 & 30.0 & 30.0 & 180.0 \\
\hline & - Dryer & $\mathrm{E}$ & & Lot & 180.0 & 180.0 & 36.0 & 36.0 & 216.0 \\
\hline & - Soil Day Tank & $E$ & & 1 & 30.0 & 30.0 & 8.0 & 8.0 & 38.0 \\
\hline & - Soil Storage Silos & E & & 2 & 65.0 & 130.0 & 20.0 & 40.0 & 170.0 \\
\hline & - Soil Transfer Pump & $\mathrm{E}$ & & 1 & 30.0 & 30.0 & 10.0 & 10.0 & 40.0 \\
\hline & - Slag Cooling \& Packaging (A-VITRF) & & & & & & & & \\
\hline & - Metal Canister Capper & F & & 1 & 150.0 & 150.0 & 30.0 & 30.0 & 180.0 \\
\hline & - Canister Decon Unit & F & & 1 & 70.0 & 70.0 & 9.0 & 9.0 & 79.0 \\
\hline & - Canister Cooling System & F & & 2 & 75.0 & 150.0 & 40.0 & 80.0 & 230.0 \\
\hline & - Canister Conveyor & E & & 30 & 4.0 & 120.0 & 1.0 & 30.0 & 150.0 \\
\hline & - Grouting Unit & E & & 1 & 150.0 & 150.0 & 25.0 & 25.0 & 175.0 \\
\hline & - Air Pollution Control & & & & & & & & \\
\hline & - Quencher & $E$ & & 1 & 200.0 & 200.0 & 4.0 & 4.0 & 204.0 \\
\hline & - Ceramic Filters & $\mathrm{E}$ & & 2 & 240.0 & 480.0 & 48.0 & 96.0 & 576.0 \\
\hline & - Primary Activated Carbon Filter & $\mathrm{E}$ & & Lot & 50.0 & 50.0 & 1.0 & 1.0 & 51.0 \\
\hline & - Hepa Filter & $\mathrm{E}$ & & 2 & 170.0 & 340.0 & 65.0 & 130.0 & 470. \\
\hline & - Induced Fan & E & & 2 & 15.0 & 30.0 & 3.0 & 6.0 & 36.8 \\
\hline & - Baghouse & $\mathbf{E}$ & & & & & & & \\
\hline & Stack & $E$ & & 1 & 20.0 & 20.0 & 4.0 & 4.0 & 24.1 \\
\hline
\end{tabular}


Table B-1.1: Equipment Purchase \& Installation Budget Estimates

Enhanced Non-Thermal Treatment System

ENTS - 1 CWO, Desorption and Vitrification

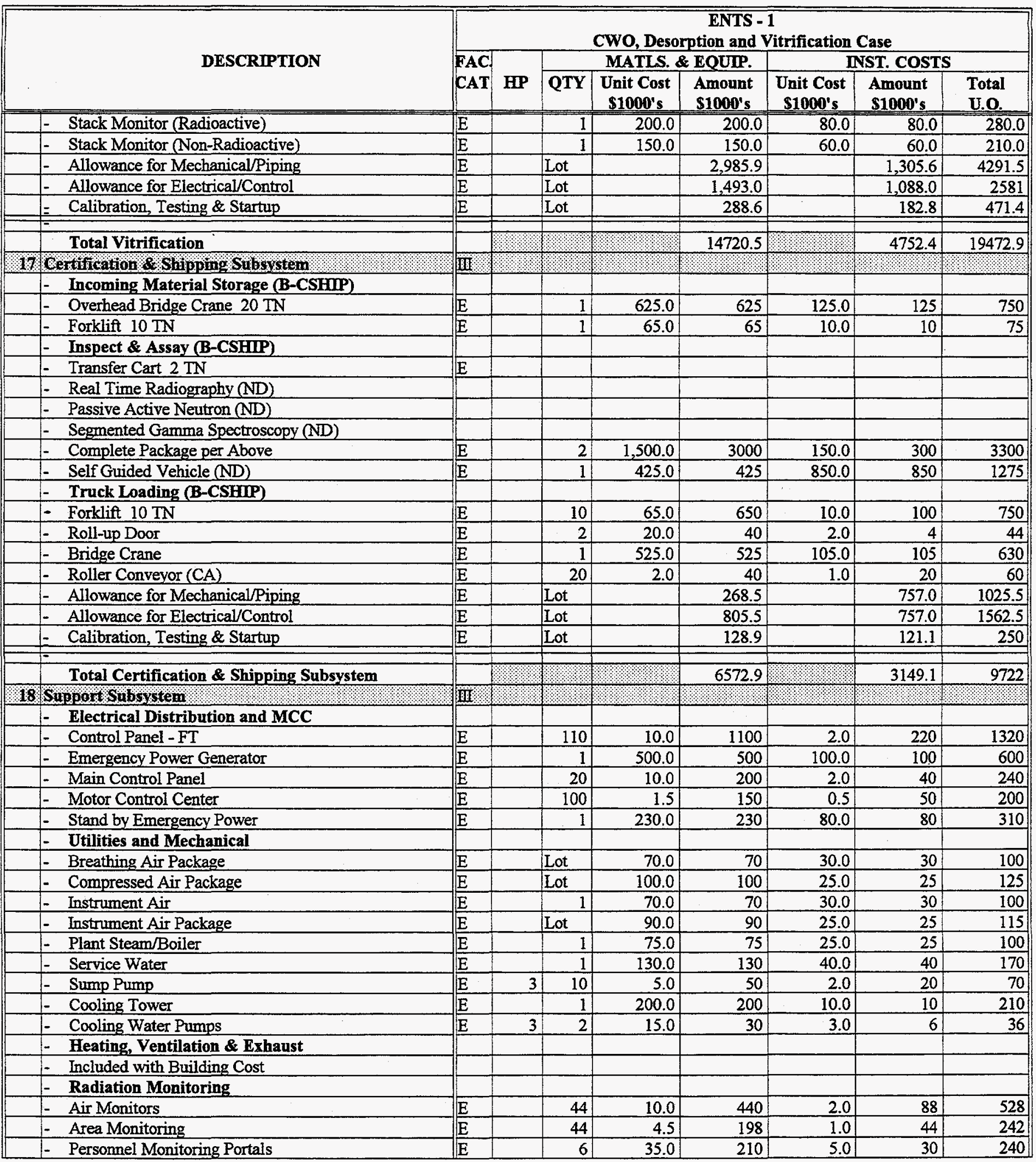


Table B-1.1: Equipment Purchase \& Installation Budget Estimates

Enhanced Non-Thermal Treatment System

ENTS - 1 CWO, Desorption and Vitrification

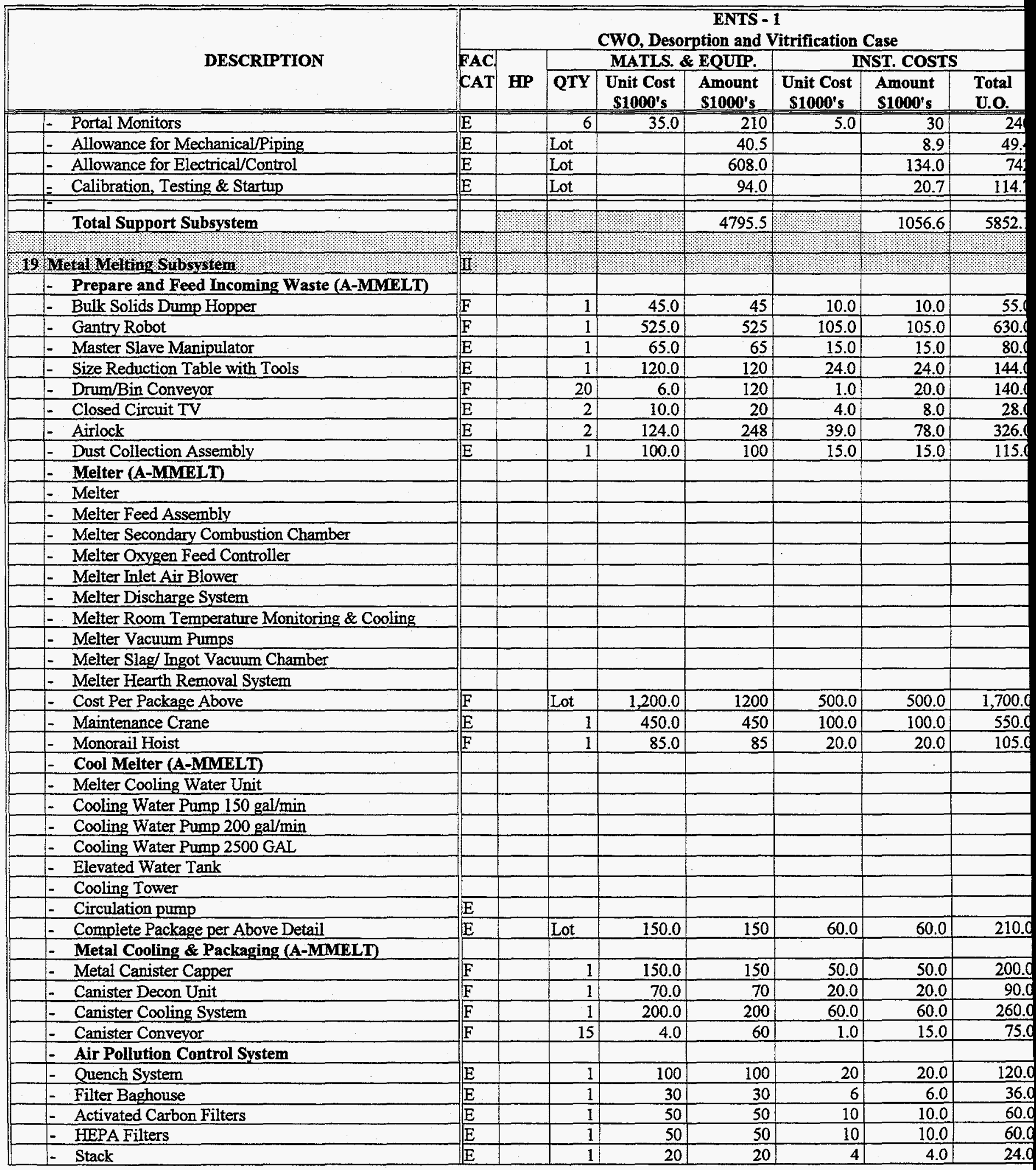


Table B-1.1: Equipment Purchase \& Installation Budget Estimates

Enhanced Non-Thermal Treatment System

ENTS - 1 CWO, Desorption and Vitrification

\begin{tabular}{|c|c|c|c|c|c|c|c|c|}
\hline \multirow{3}{*}{ DESCRIPTION } & \multicolumn{8}{|c|}{$\begin{array}{c}\text { ENTS - } 1 \\
\text { CWO, Desorption and Vitrification Case }\end{array}$} \\
\hline & \multirow{2}{*}{$\begin{array}{l}\mathrm{FAC} \\
\mathrm{CAT}\end{array}$} & \multirow{2}{*}{ HP } & \multicolumn{3}{|c|}{ MATLS. \& EQUIP. } & \multicolumn{3}{|c|}{ INST. COSTS } \\
\hline & & & QTY & $\begin{array}{l}\text { Unit Cost } \\
\$ 1000^{\prime} s\end{array}$ & $\begin{array}{l}\text { Amount } \\
\text { S1000's }\end{array}$ & $\begin{array}{l}\text { Unit Cost } \\
\text { S1000's }\end{array}$ & $\begin{array}{l}\text { Amount } \\
\text { S1000's }\end{array}$ & $\begin{array}{l}\text { Total } \\
\text { U.O. }\end{array}$ \\
\hline - Stack Monitor (Radioactive) & $E$ & & 1 & 200 & 200 & 80 & 80.0 & 280.0 \\
\hline - Stack Monitor (Non-Radioactive) & $\mathrm{E}$ & & 1 & 150 & 150 & 60 & 60.0 & 210.0 \\
\hline - Allowance for Mechanical/Piping & $E$ & & Lot & & $1,262.4$ & & 774.0 & 2036.4 \\
\hline - Allowance for Electrical/Control & $E$ & & Lot & & 631.2 & & 645.0 & 1276.2 \\
\hline - Calibration, Testing \& Startup & $\mathrm{E}$ & & Lot & & 122.0 & & 108.4 & 230.4 \\
\hline & & & & & & & & \\
\hline Total Metal Melting Subsystem & & & & & $6,223.6$ & & $2,817.4$ & $9,041.0$ \\
\hline
\end{tabular}


Table B-1.2 : Development, Testing \& Evaluation Cost Estimate ENTS - 1 CWO, Desorption and Vitrification

\begin{tabular}{|c|c|c|c|c|c|c|c|c|c|c|}
\hline & \multirow[b]{2}{*}{ UNIT OPERATION } & \multicolumn{9}{|c|}{$\begin{array}{c}\text { ENTS - } 1 \\
\text { CWO, Desorption and Vitrification }\end{array}$} \\
\hline & & $\begin{array}{c}\text { Primaxy } \\
\text { Paper } \\
\text { Evaluation } \\
\text { Research } \\
\text { Manpower }\end{array}$ & \begin{tabular}{|c|} 
Bench \\
Scale \\
Studies \\
Research \\
Manpower \\
\end{tabular} & \begin{tabular}{|c|} 
Sec. \\
Paper \\
Evaluation \\
Research \\
Manpower \\
\end{tabular} & $\begin{array}{c}\text { Total } \\
\text { Research } \\
\text { Manpower }\end{array}$ & $\begin{array}{c}\text { Bench } \\
\text { Scale } \\
\text { Studies } \\
\text { Equipment } \\
\$ 1000 \\
\end{array}$ & $\begin{array}{c}\text { Bench } \\
\text { Scale } \\
\text { Studies } \\
\text { Installation } \\
\$ 1000 \\
\end{array}$ & \begin{tabular}{|c|} 
Pilot \\
Plant \\
Mock-up \\
Test Demo. \\
Manpower \\
\end{tabular} & $\begin{array}{c}\text { Pilot } \\
\text { Plant } \\
\text { Mock-up } \\
\text { Test Bldg. } \\
\$ 1000 \\
\end{array}$ & $\begin{array}{c}\text { Pilot } \\
\text { Plant } \\
\text { Mock-up } \\
\text { Test Equip. } \\
\text { \$1000 } \\
\end{array}$ \\
\hline \multicolumn{11}{|c|}{1 Administration Building Subsystem } \\
\hline 2 & Receiving \& Inspection Subsystem & 12 & & & 12 & & & 25 & 1,200 & 6,000 \\
\hline 3 & Aqueous Waste Treatment Subsystem & 5 & 3 & 3 & 11 & 100 & 300 & 10 & 400 & 2,000 \\
\hline 4 & Organic Destruction Subsystem & 18 & 20 & 4 & 42 & 500 & 150 & 30 & 400 & 4,000 \\
\hline 5 & Residue \& Sludge Treatment Subsystem & 13 & 8 & 4 & 25 & 500 & 150 & 30 & 500 & 2,000 \\
\hline \multicolumn{11}{|c|}{6 Soft Debris Treatment Subsystem } \\
\hline \multicolumn{11}{|c|}{7 Complex Debris Treatment Subsystem } \\
\hline 8 & Open Debris Treatment Subsystem & 2 & 6 & 2 & 10 & 300 & 50 & 20 & 1,000 & 3,000 \\
\hline 9 & Bulk Soil Treatment Subsystem & 10 & 6. & 2 & 18 & 200 & 50 & 10 & 200 & 500 \\
\hline \multicolumn{11}{|c|}{10 Metal Decontamination Subsystem } \\
\hline 11 & Special Waste & 8 & 20 & 5 & 33 & 600 & 250 & 20 & 400 & 2,000 \\
\hline 12 & Mercury Amalgamation Subsystem & 1 & 3 & & 4 & 100 & 50 & & & \\
\hline 13 & Lead Recovery Subsystem & 2 & 2 & & 4 & 100 & 30 & & & \\
\hline 14 & Air Pollution Control Subsystem & 5 & 3 & 2 & 10 & 200 & 50 & 15 & 400 & 2,000 \\
\hline 15 & Polymer Stabilization Subsystem & 2 & 8 & 4 & 14 & 300 & 50 & 15 & 400 & 2,000 \\
\hline 16 & Vitrification & 2 & 8 & 4 & 14 & 300 & 50 & 15 & 400 & 1,000 \\
\hline \multicolumn{11}{|c|}{17 Certification \& Shipping Subsystem } \\
\hline 18 & Support Subsystem & 5 & & & 5 & & & & & \\
\hline \multirow[t]{4}{*}{19} & Metal Melting Subsystem & & & & & & & & & \\
\hline & Unit Cost (\$/Unit) & & & & $\$ 150,000$ & & & $\$ 150,000$ & & \\
\hline & Total Cost & & & & 30,300 & 3,200 & 1,180 & 28,500 & 5,300 & 24,500 \\
\hline & & Post Totals To? & Table 4-1, Item & & 1.1 & 1.2 & 1.3 & 2.1 & 2.5 .1 & 2.5 .2 \\
\hline
\end{tabular}


Table B-1.3 : Building, Equipment and Installation Cost Summary ENTS - 1 CWO, Desorption and Vitrification

\begin{tabular}{|c|c|c|c|c|c|c|c|c|c|c|}
\hline & \multirow{3}{*}{ UNIT OPERATION } & \multicolumn{9}{|c|}{$\begin{array}{c}\text { ENTS - 1 } \\
\text { CWO, Desorption and Vitrification }\end{array}$} \\
\hline & & \multicolumn{5}{|c|}{ Building Area } & \multicolumn{3}{|c|}{\begin{tabular}{|c|} 
Material \& Equipment Costs \\
\end{tabular}} & \multirow{2}{*}{$\begin{array}{c}\text { Total } \\
\text { Cost per } \\
\text { Unit Oprtn. } \\
\$ 1000 \\
\end{array}$} \\
\hline & & $\begin{array}{c}\text { Cost } \\
\text { Category } 1 \\
\text { sq.ft } \\
\end{array}$ & $\begin{array}{c}\text { Cost } \\
\text { Category } 2 \\
\text { sq.ft }\end{array}$ & $\begin{array}{c}\text { Cost } \\
\text { Category } 3 \\
\text { sq.ft } \\
\end{array}$ & $\begin{array}{c}\text { Cost } \\
\text { Category } 4 \\
\text { sq.ft } \\
\end{array}$ & $\begin{array}{c}\begin{array}{c}\text { Total } \\
\text { Area Cost } \\
\mathbf{S 1 0 0 0}\end{array} \\
\end{array}$ & $\begin{array}{c}\text { Purchase } \\
\text { Cost } \\
\$ 1000 \\
\end{array}$ & 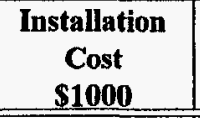 & $\begin{array}{c}\text { Total } \\
\text { Cost } \\
\$ 1000 \\
\end{array}$ & \\
\hline 1.0 & Administration Building Subsystem & 12,440 & 3,000 & & & $3,499.0$ & $3,162.7$ & 888.2 & $4,051.0$ & $7,550.0$ \\
\hline 2.0 & Receiving \& Inspection Subsystem & & 24,227 & 2,970 & 14,839 & $38,966.0$ & $18,493.5$ & $6,774.6$ & $25,268.0$ & $64,234.0$ \\
\hline 3.0 & Aqueous Waste Treatment Subsystem & & 1,150 & 1,751 & 1,590 & $5,287.0$ & $4,980.2$ & $1,516.1$ & $6,496.0$ & $11,783.0$ \\
\hline 4.0 & Organic Destruction Subsystem & & 1,128 & 1,717 & 1,560 & $5,187.0$ & $5,750.4$ & $1,476.4$ & $7,227.0$ & $12,414.0$ \\
\hline 5.0 & Residue \& Sludge Treatment Subsystem & & 2,040 & 3,105 & 2,820 & $9,376.0$ & $4,892.5$ & $1,168.4$ & $6,061.0$ & $15,437.0$ \\
\hline 6.0 & Soft Debris Treatment Subsystem & & 1,606 & 2,444 & 2,220 & $7,381.0$ & $8,590.0$ & $1,454.1$ & $10,044.0$ & $17,425.0$ \\
\hline 7.0 & Complex Debris Treatment Subsystem & & & & & & & & & \\
\hline 8.0 & Open Debris Treatment Subsystem & & 1,888 & 2,873 & 2,610 & $8,678.0$ & $2,052.9$ & 670.5 & $2,723.0$ & $11,401.0$ \\
\hline 9.0 & Bulk Soil Treatment Subsystem & & 1,606 & 2,444 & 2,220 & $7,381.0$ & $2,822.3$ & 832.4 & $3,655.0$ & $11,036.0$ \\
\hline 10.0 & Metal Decontamination Subsystem & & 641 & 169 & 1,904 & $3,709.0$ & $3,571.6$ & $1,495.5$ & $5,067.0$ & $8,776.0$ \\
\hline 11.0 & Special Waste & & 604 & 159 & 1,792 & $3,491.0$ & & & & $3,491.0$ \\
\hline 12.0 & Mercury Amalgamation Subsystem & & 679 & 179 & 2,016 & $3,928.0$ & $2,895.9$ & $1,048.6$ & $3,945.0$ & $7,873.0$ \\
\hline 13.0 & Lead Recovery Subsystem & & 830 & 219 & 2,464 & $4,800.0$ & $4,027.4$ & $1,154.9$ & $5,182.0$ & $9,982.0$ \\
\hline 14.0 & Air Pollution Control Subsystem & & 955 & 1,453 & 1,320 & $4,389.0$ & $2,400.8$ & 884.5 & $3,285.0$ & $7,674.0$ \\
\hline 15.0 & Polymer Stabilization Subsystem & & 2,322 & 3,534 & 3,210 & $10,673.0$ & $5,187.3$ & $1,511.6$ & $6,699.0$ & $17,372.0$ \\
\hline 16.0 & Vitrification & & 2,614 & 8,209 & 4,140 & $17,987.0$ & $14,720.5$ & $4,752.4$ & $19,473.0$ & $37,460.0$ \\
\hline 17.0 & Certification \& Shipping Subsystem & & 28,277 & & & $11,876.0$ & $6,572.9$ & $3,149.1$ & $9,722.0$ & $21,598.0$ \\
\hline 18.0 & Support Subsystem & 3,360 & 16,883 & & & $7,696.0$ & $4,795.5$ & $1,056.6$ & $5,852.0$ & $13,548.0$ \\
\hline \multirow[t]{3}{*}{19.0} & Metal Melting Subsystem & & 1,169 & 309 & 3,472 & $6,764.0$ & $6,223.6$ & $2,817.4$ & $9,041.0$ & $15,805.0$ \\
\hline & Total Cost & & & & & $161,068.0$ & & & $133,791.0$ & $294,859.0$ \\
\hline & & \multicolumn{4}{|c|}{ Post Totals To Table 4-1, Item } & 3.4 .1 & & & 3.4 .2 & \\
\hline
\end{tabular}




\begin{tabular}{|c|c|c|c|c|c|c|c|}
\hline & \multirow[b]{2}{*}{ UNIT OPERATION } & \multicolumn{6}{|c|}{$\begin{array}{c}\text { ENTS - } 1 \\
\text { CWO, Desorption and Vitrification }\end{array}$} \\
\hline & & $\begin{array}{l}\text { Operating } \\
\text { FTE }\end{array}$ & $\begin{array}{l}\text { Utilities } \\
\$ 1000 \\
\end{array}$ & $\begin{array}{c}\text { Materials } \\
\$ 1000 \\
\end{array}$ & \begin{tabular}{|c|} 
Maintenance \\
Labor (1) \\
$\$ 1000$ \\
\end{tabular} & $\begin{array}{c}\text { Maintenance } \\
\text { Materials (2) } \\
\$ 1000 \\
\end{array}$ & $\begin{array}{l}\text { Totals } \\
\$ 1000 \\
\end{array}$ \\
\hline 1 & Administration Building Subsystem & 32 & 5 & 164 & 318 & 127 & 445 \\
\hline 2 & Receiving \& Inspection Subsystem & 170 & 159 & 612 & 1,850 & 740 & 2,590 \\
\hline 3 & Aqueous Waste Treatment Subsystem & 5 & 300 & 1,705 & 873 & 349 & 1,222 \\
\hline 4 & Organic Destruction Subsystem & 12 & 298 & 297 & 1,438 & 575 & 2,013 \\
\hline 5 & Residue \& Sludge Treatment Subsystem & 8 & 21 & 1 & 855 & 342 & 1,197 \\
\hline 6 & Soft Debris Treatment Subsystem & 14 & 708 & 704 & 2,148 & 859 & 3,007 \\
\hline 7 & Complex Debris Treatment Subsystem & & & & & & \\
\hline 8 & Open Debris Treatment Subsystem & 8 & 25 & 100 & 360 & 144 & 504 \\
\hline 9 & Bulk Soil Treatment Subsystem & 2 & 22 & 14 & 140 & 56 & 196 \\
\hline 10 & Metal Decontamination Subsystem & 4 & 5 & 25 & 625 & 250 & 875 \\
\hline 11 & Special Waste & 3 & 12 & 200 & & & \\
\hline 12 & Mercury Amalgamation Subsystem & 4 & 2 & 10 & 508 & 203 & 711 \\
\hline 13 & Lead Recovery Subsystem & 8 & 73 & 50 & 705 & 282 & 987 \\
\hline 14 & Air Pollution Control Subsystem & 4 & 67 & 130 & 420 & 168 & 588 \\
\hline 15 & Polymer Stabilization Subsystem & 38 & 118 & 1,858 & 908 & 363 & 1,271 \\
\hline 16 & Vitrification & 16 & 37 & 661 & 1,473 & 589 & 2,062 \\
\hline 17 & Certification \& Shipping Subsystem & 67 & 12 & 70 & 658 & 263 & 921 \\
\hline 18 & Support Subsystem & 5 & & & 480 & 192 & 672 \\
\hline 19 & Metal Melting Subsystem & 5 & 79 & 33 & 623 & 249 & 872 \\
\hline & Unit cost (\$/unit) & $\$ 140,000$ & & & & & \\
\hline & Total Cost & 56,630 & 1943 & 6,634 & & & 20,133 \\
\hline & Post Totals To Table 4-1, Item & 5.1 & 5.2 & 5.3 & & & 5.4 \\
\hline
\end{tabular}

Notes:

1. Annual Maintenance Labor is $250 \%$ of maintenance material cost.

2. Maintenance Material is assumed to be $2 \%$ of equipment capital cost for the Bulk Soil Treatment subsystem, because the capital equipment is based on one shift operation.

3. Maintenance Material is assumed to be $10 \%$ of equipment capital cost for organic destruction and soft debris.

4. Maintenance Material is assumed to be $4 \%$ of equipment capital cost for Administration, Receiving \& Inspection,

Grout Stabilization, Certification \& Shipping, and Support subsystems.

5. Maintenance Material is assumed to be $7 \%$ of equipment capital cost for all other subsystems 
Table B-1.5 : Decontamination \& Decommissioning Costs ENTS - 1 CWO, Desorption and Vitrification

\begin{tabular}{|c|c|c|c|c|c|c|}
\hline & \multirow{3}{*}{ UNIT OPERATION } & \multicolumn{5}{|c|}{$\begin{array}{c}\text { ENTS - 1 } \\
\text { CWO, Desorption and Vitrification }\end{array}$} \\
\hline & & \multirow[b]{2}{*}{$\begin{array}{c}\text { Cost } \\
\text { Category } 1 \\
\text { sq.ft }\end{array}$} & \multicolumn{3}{|c|}{ Building Area } & \multirow[b]{2}{*}{$\begin{array}{c}\text { Total } \\
\text { Area Cost } \\
\$ 1000\end{array}$} \\
\hline & & & $\begin{array}{c}\text { Cost } \\
\text { Category } 2 \\
\text { sq.ft }\end{array}$ & $\begin{array}{c}\text { Cost } \\
\text { Category } 3 \\
\text { sq.ft }\end{array}$ & $\begin{array}{c}\text { Cost } \\
\text { Category } 4 \\
\text { sq.ft }\end{array}$ & \\
\hline 1 & Administration Building Subsystem & 12,440 & 3,000 & & & 6,948 \\
\hline 2 & Receiving \& Inspection Subsystem & & 24,227 & 2,970 & 14,839 & 18,916 \\
\hline 3 & Aqueous Waste Treatment Subsystem & & 1,150 & 1,751 & 1,590 & 2,021 \\
\hline 4 & Organic Destruction Subsystem & & 1,128 & 1,717 & 1,560 & 1,983 \\
\hline 5 & Residue \& Sludge Treatment Subsystem & & 2,040 & 3,105 & 2,820 & 3,584 \\
\hline 6 & Soft Debris Treatment Subsystem & & 1,606 & 2,444 & 2,220 & 2,822 \\
\hline 7 & Complex Debris Treatment Subsystem & & & & & \\
\hline 8 & Open Debris Treatment Subsystem & & 1,888 & 2,873 & 2,610 & 3,317 \\
\hline 9 & Bulk Soil Treatment Subsystem & & 1,606 & 2,444 & 2,220 & 2,822 \\
\hline 10 & Metal Decontamination Subsystem & & 641 & 169 & 1,904 & 1,221 \\
\hline 11 & Special Waste & & 604 & 159 & 1,792 & 1,150 \\
\hline 12 & Mercury Amalgamation Subsystem & & 679 & 179 & 2,016 & 1,293 \\
\hline 13 & Lead Recovery Subsystem & & 830 & 219 & 2,464 & 1,581 \\
\hline 14 & Air Pollution Control Subsystem & & 955 & 1,453 & 1,320 & 1,678 \\
\hline 15 & Polymer Stabilization Subsystem & & 2,322 & 3,534 & 3,210 & 4,080 \\
\hline 16 & Vitrification & & 2,614 & 8,209 & 4,140 & 6,733 \\
\hline 17 & Certification \& Shipping Subsystem & & 28,277 & & & 12,725 \\
\hline 18 & Support Subsystem & 3,360 & 16,883 & & & 9,109 \\
\hline 19 & Metal Melting Subsystem & & 1,169 & 309 & 3,472 & 2,228 \\
\hline & Total Cost & & & & & 84,211 \\
\hline & & & Oost Totals To I & able 4-1, Item & & 7.0 \\
\hline
\end{tabular}




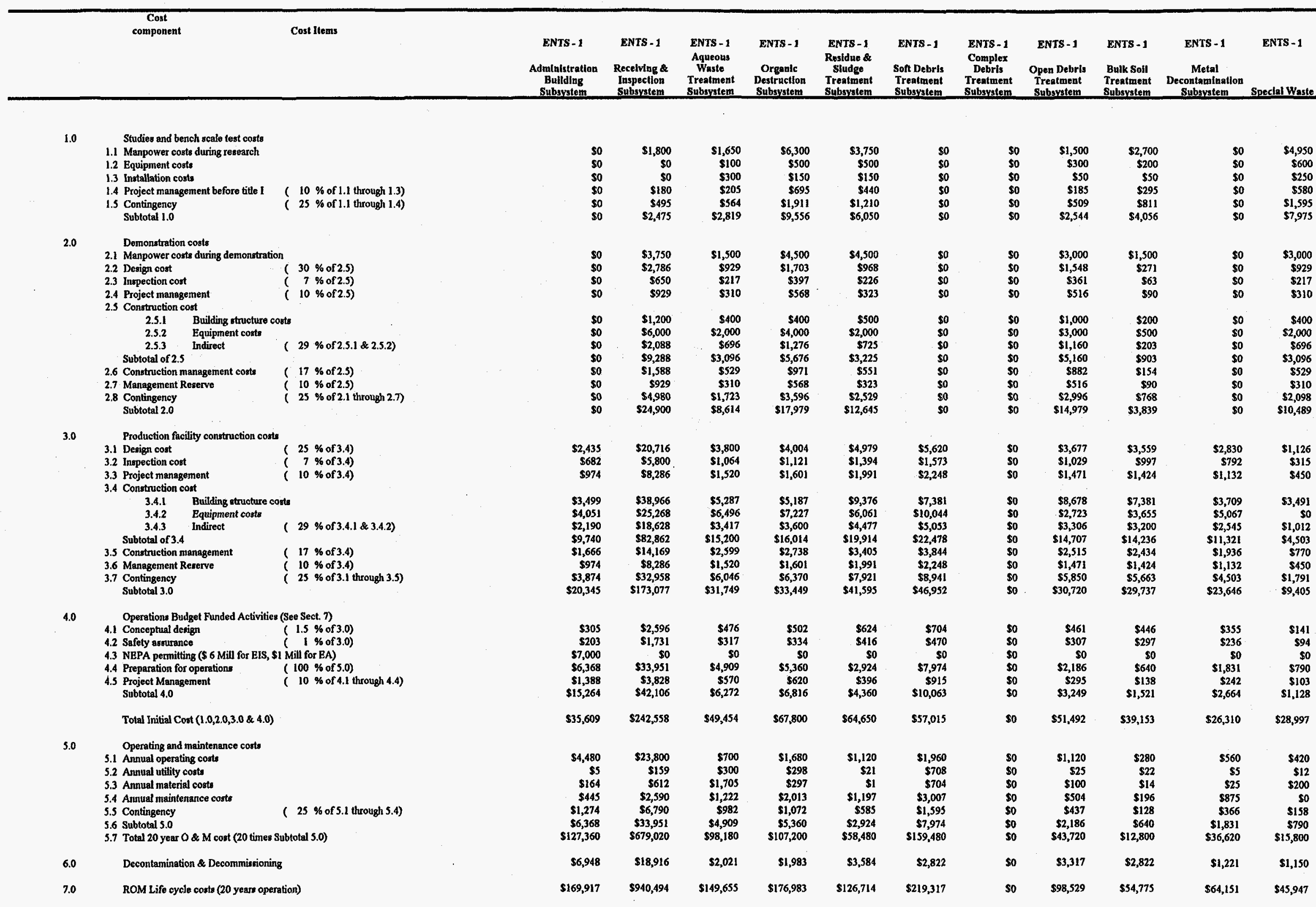


Table B-1.6 : Total Life-Cycle Cost (without Disposal) Estimate Summary for Enhanced Integrated Non Thermal Treatment Systems (cost module ENTS-1)

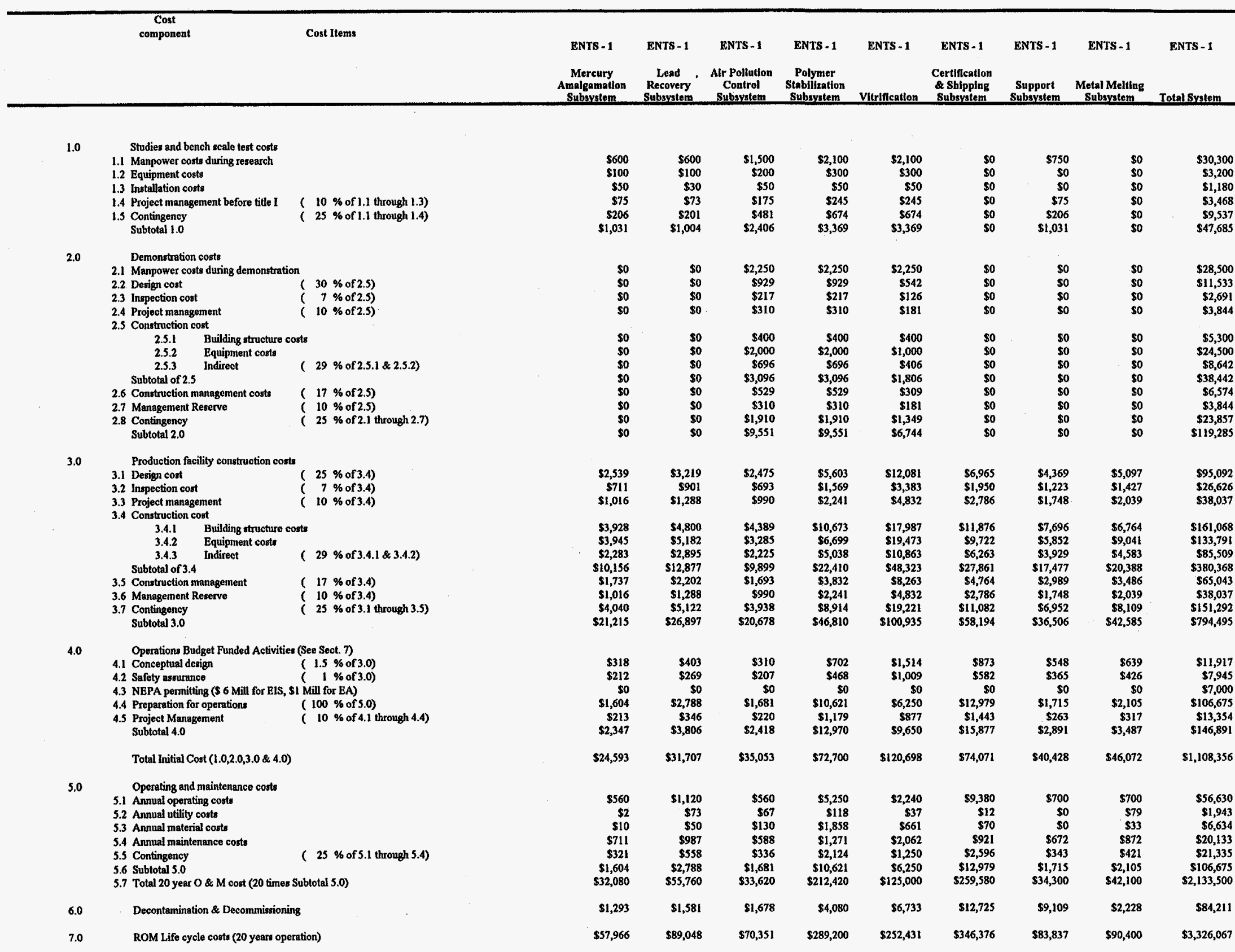




\section{B-2 ENTS-2 Cost Tables}

B-2.1. Equipment Purchase and Installation Budget Estimates

B-2.2. Development, Testing and Evaluation Cost Estimate

B-2.3. Building, Equipment and Installation Cost Summary

B-2.4. Annual Operating and Maintenance Costs

B-2.5. Decontamination and Decommissioning Costs

B-2.6 Total Life-Cycle Cost (without Disposal) Estimate Summary for Enhanced Integrated Nonthermal Treatment Systems 
Table B-2.1: Equipment Purchase and Installation Budget Estimates

Enhanced Non-Thermal Treatment System

ENTS - 2 CWO and Vitrification

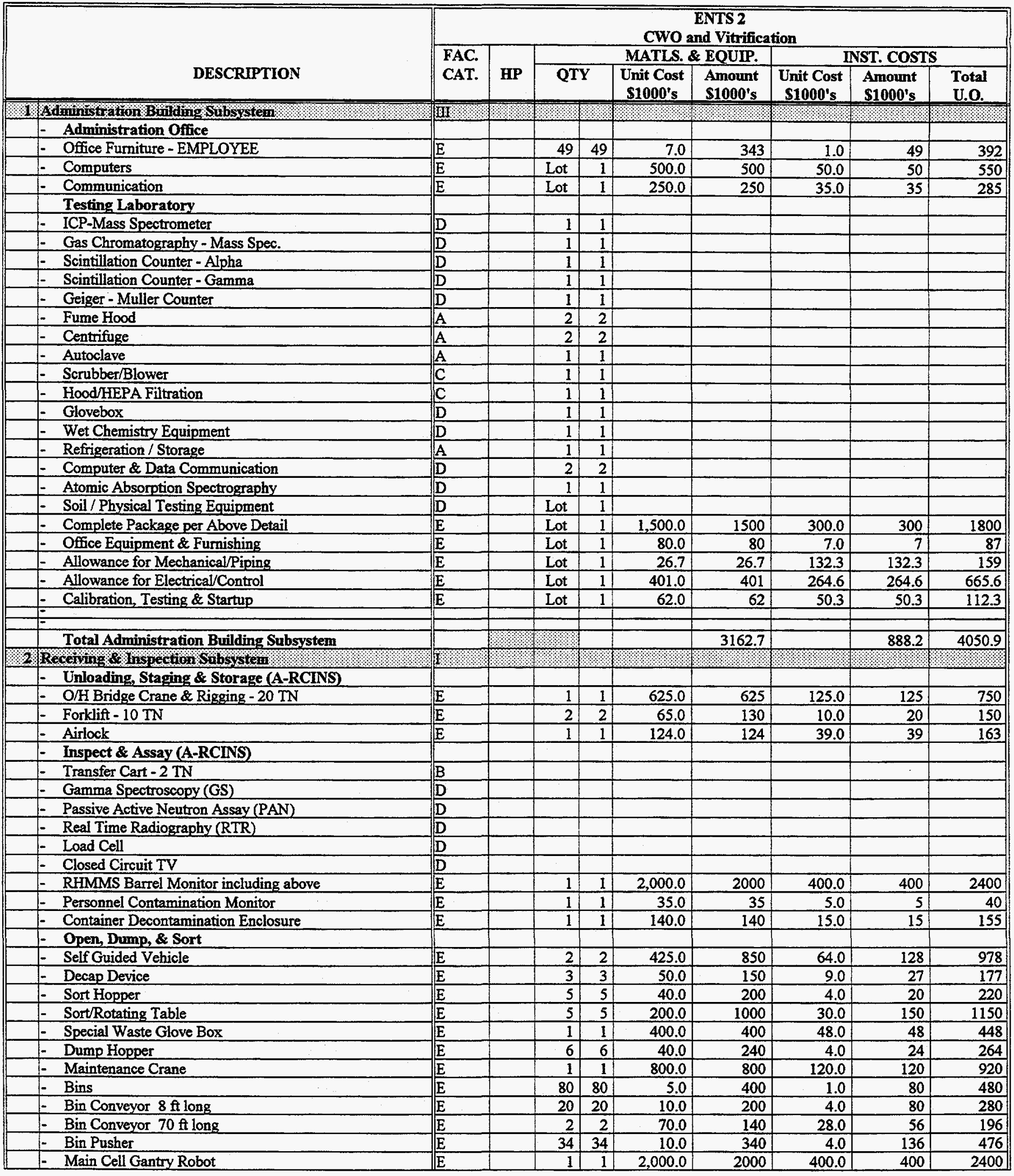


Table B-2.1: Equipment Purchase and Installation Budget Estimates

Enhanced Non-Thermal Treatment System

ENTS - 2 CWO and Vitrification

\begin{tabular}{|c|c|c|c|c|c|c|c|c|c|c|}
\hline \multirow{3}{*}{\multicolumn{2}{|c|}{ DESCRIPTION }} & \multicolumn{9}{|c|}{$\begin{array}{c}\text { ENTS 2 } \\
\text { CWO and Vitrification }\end{array}$} \\
\hline & & \multirow{2}{*}{$\begin{array}{l}\text { FAC. } \\
\text { CAT. }\end{array}$} & \multirow[b]{2}{*}{ HP } & \multicolumn{4}{|c|}{ MATLS. \& EQUIP. } & \multicolumn{3}{|c|}{ INST. COSTS } \\
\hline & & & & $\overline{\mathrm{QTY}}$ & & $\begin{array}{l}\text { Unit Cost } \\
\text { S1000's }\end{array}$ & $\begin{array}{l}\text { Amount } \\
\text { S1000's }\end{array}$ & $\begin{array}{c}\text { Unit Cost } \\
\text { S1000's }\end{array}$ & $\begin{array}{l}\text { Amount } \\
\text { S1000's }\end{array}$ & $\begin{array}{l}\text { Total } \\
\text { U.o. }\end{array}$ \\
\hline & - Sorting Table Hydraulic Manipulator & $E$ & & 2 & 2 & 120.0 & 240 & 40.0 & 80 & 320 \\
\hline & - Sorting Master Slave Manipulator & E & & 5 & 5 & 100.0 & 500 & 40.0 & 200 & 700 \\
\hline & - Box Size Reduction Unit & $E$ & & 1 & 1 & $1,500.0$ & 1500 & 400.0 & 400 & 1900 \\
\hline & - Drum Opening Gantry Robot & $E$ & & 2 & 2 & 400.0 & 800 & 80.0 & 160 & 960 \\
\hline & - Glovebox Hydraulic Manipulator & $E$ & & 2 & 2 & 120.0 & 240 & 40.0 & 80 & 320 \\
\hline & - Container Grappler & $E$ & & 4 & 4 & 50.0 & 200 & 8.0 & 32 & 232 \\
\hline & - Decap Enclosure & $E$ & & 3 & 3 & 100.0 & 300 & 30.0 & 90 & 390 \\
\hline & - Bin Hoist / Pusher & $B$ & 5 & 1 & 1 & 33 & 66 & 8 & 8 & 74 \\
\hline & - Discharge. \& Oversize Load Hopper (100 CF) & $B$ & 1 & 1 & 1 & 25 & 50 & 7 & 7 & 57 \\
\hline & - Primary Shredder $(25,1.5,2$ Tons $/ \mathrm{hr})$ & $E$ & 250 & 1 & 1 & 375 & 375 & 75 & 75 & 450 \\
\hline & - Secondary Shredder $(.5,1.5,3$ Tons $/ \mathrm{hr})$ & E & 60 & 1 & 1 & 160 & 160 & 32 & 32 & 192 \\
\hline & - Live Bottom Bulk Storage & $B$ & 1 & 1 & 1 & 25 & 50 & 7 & 7 & 57 \\
\hline & - Conveyor / Canister Handler & $B$ & 5 & 1 & 1 & 40 & 80 & 10 & 10 & 90 \\
\hline & - Dust Collection System w/ Blower & E & & Lot & 1 & 108 & 108 & 17 & 17 & 125 \\
\hline & - HEPA Filter & E & & 2 & 2 & 25 & 50 & 5 & 10 & 60 \\
\hline & - Closed Circuit TV & E & & 8. & 8 & 10.0 & 80 & 2.0 & 16 & 96 \\
\hline & - Airlock & $E$ & & 4 & 4 & 124.0 & 496 & 39.0 & 156 & 652 \\
\hline & - Roll up Door (CA) & $\mathrm{E}$ & & 2 & 2 & 20.0 & 40 & 2.0 & 4 & 44 \\
\hline & - Allowance for Mechanical/Piping & E & & Lot & 1 & 755.5 & 755.5 & $1,628.5$ & 1628.5 & 2384 \\
\hline & - Allowance for Electrical/Control & $E$ & & Lot & 1 & $2,266.4$ & 2266.4 & $1,628.5$ & 1628.5 & 3894.9 \\
\hline & $=$ Calibration, Testing \& Startup & $E$ & & Lot & 1 & 362.6 & 362.6 & 260.6 & 260.6 & 623.2 \\
\hline & Total Receiving \& Inspection Subsystem & & & & & & 18493.5 & & 6774.6 & 25268.1 \\
\hline 3 & Aqueous Waste Tre thent Subs stem $/$ & 1 & & 3 & & 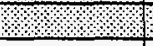 & $1 \%$ & & & 10 \\
\hline & - Batch Liquid Waste (A-AQWTR) & & & & & & & & & \\
\hline & - High TOC Batch Tank with Agitator & $E$ & & 1 & 1 & 27.0 & 27 & 5.0 & 5 & 32 \\
\hline & - High TOC Batch Tank Pump & E & & 1 & 1 & 5.0 & 5 & 1.0 & 1 & 6 \\
\hline & - Low IDS Batch Tank with Agitator & E & & 1 & 1 & 27.0 & 27 & 5.0 & 5 & 32 \\
\hline & - Low TDS Batch Tank Pump & $E$ & & 1 & 1 & 5.0 & 5 & 1.0 & 1 & 6 \\
\hline & - High TDS/ Heavy Metals Batch Tank with Agitator & $E$ & & 1 & 1 & 27.0 & 27 & 5.0 & 5 & 32 \\
\hline & - High TDS/ Heavy Metals Batch Tank Pump & $E$ & & 1 & 1 & 5.0 & 5 & 1.0 & 1 & 6 \\
\hline & - Hg Batch Tank with Agitator & $E$ & & 1 & 1 & 27.0 & 27 & 5.0 & 5 & 32 \\
\hline & - Hg Batch Tank Pump & E & & 1 & 1 & 5.0 & 5 & 1.0 & 1 & 6 \\
\hline & - Airlocks & $E$ & & 2 & 2 & 124.0 & 248 & 39.0 & 78 & 326 \\
\hline & - Closed Circuit TV & E & & 5 & 5 & 10.0 & 50 & 2.0 & 10 & 60 \\
\hline & - Crane & $E$ & & 1. & 1 & 525.0 & 525 & 105.0 & 105 & 630 \\
\hline & - Shield Window & $E$ & & 1 & 1 & 130 & 130 & 44 & 44 & 174 \\
\hline & - Gross Organics Removal (A-AQWTR) & & & & & & & & & \\
\hline & - Oil/ Water Separator & $E$ & & 1 & 1 & 18.0 & 18 & 2.5 & 2.5 & 20.5 \\
\hline & - Neutralization / Settling (A-AQWTR) & & & & & & & & & \\
\hline & - Neutralization System & $E$ & & 1 & 1 & 95.0 & 95 & 19.0 & 19 & 114 \\
\hline & - Neutralization Overflow Pump & $E$ & & 1 & 1 & 5.0 & 5 & 1.0 & 1 & 6 \\
\hline & - Sludge Transfer Pump & E & & 1 & 1 & 5.0 & 5 & 1.0 & 1 & 6 \\
\hline & - Sludge Settler & $E$ & & 1 & 1 & 27.0 & 27 & 5.0 & 5 & 32 \\
\hline & - Sludge Settler Pump & $\mathrm{E}$ & & 1 & 1 & 5.0 & 5 & 1.0 & 1 & 6 \\
\hline & - Suspended Solids Filtration (A-AQWTR) & & & & & & & & & \\
\hline & - Suspended Solids Filter & E & & 1 & 1 & 125.0 & 125 & 25.0 & 25 & 150 \\
\hline & - Concentration (A-AQWTR) & & & & & & & & & \\
\hline & - Evaporator with Condenser & $E$ & & 2 & 2 & 300.0 & 600 & 75.0 & 150 & 750 \\
\hline & - Evaporator Liquids Pump & E & & 2 & 2 & 5.0 & 10 & 1.0 & 2 & 12 \\
\hline & - Calcium Carbonate Removal & & & & & & & & & \\
\hline & - Tank with Agitator & $\mathrm{E}$ & & 1 & 1 & 27.0 & 27 & 5.0 & 5 & 32 \\
\hline & - Transfer Pump & $\mathrm{E}$ & & 4 & 4 & 5.0 & 20 & 1.0 & 4 & 24 \\
\hline & - Decanter Centrifuge & $\mathbf{E}$ & & 1 & 1 & 450.0 & 450 & 60.0 & 60 & 510 \\
\hline & - Holding Tank & $E$ & & 1 & 1 & 7.0 & 7 & 1.5 & 1.5 & 8.5 \\
\hline & - Slurry Pump & $\mathrm{E}$ & & 1 & 1 & 5.0 & 5 & 1.0 & 1 & 6 \\
\hline & - Filter & $E$ & & 2 & 2 & 2.5 & 5 & 0.5 & 1 & 6 \\
\hline & - Dissolved Organics Removal & & & & & & & & & \\
\hline
\end{tabular}


Table B-2.1: Equipment Purchase and Installation Budget Estimates

Enhanced Non-Thermal Treatment System

ENTS - 2 CWO and Vitrification

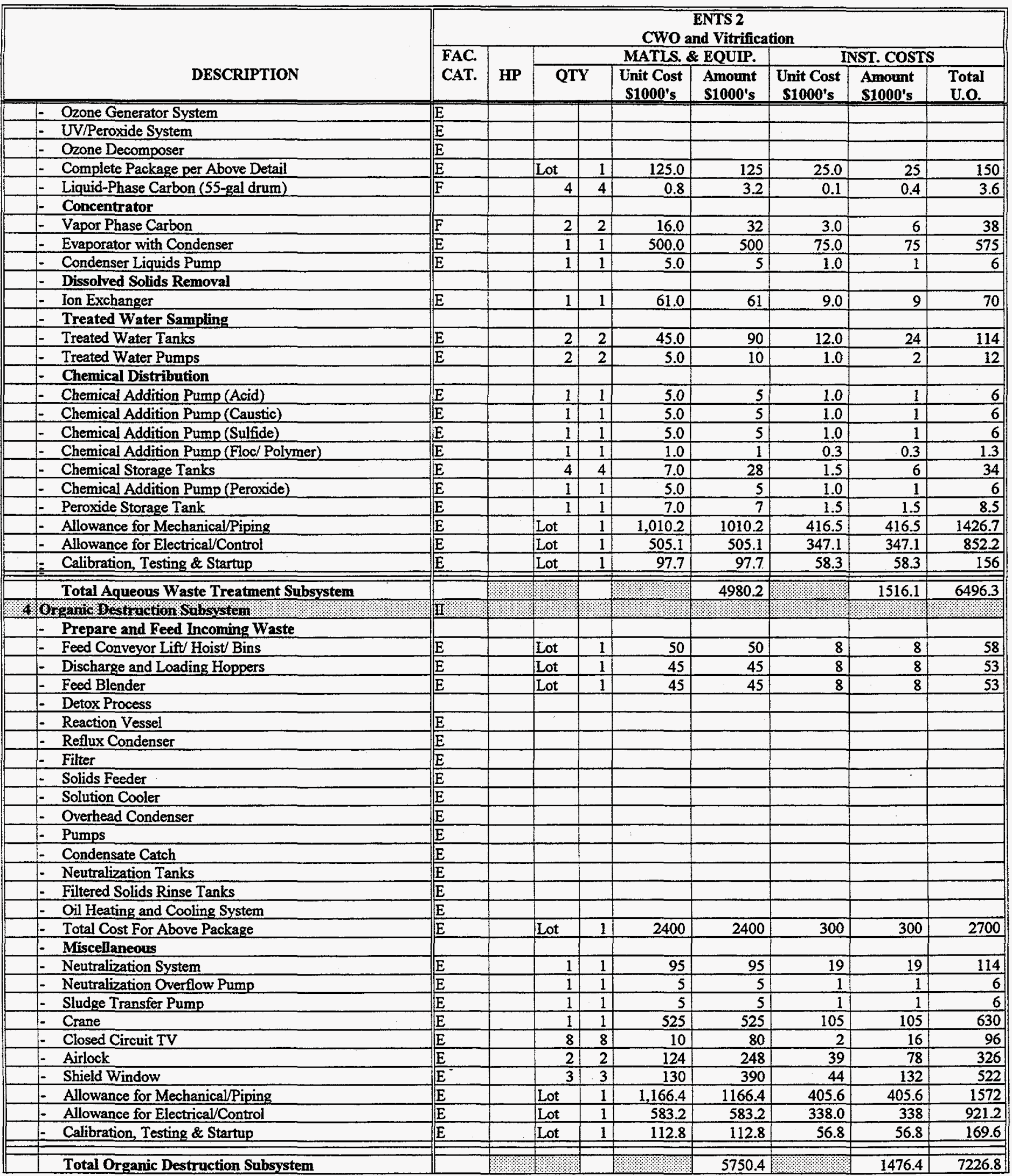


Table B-2.1: Equipment Purchase and Installation Budget Estimates

Enhanced Non-Thermal Treatment System

ENTS - 2 CWO and Vitrification

\begin{tabular}{|c|c|c|c|c|c|c|c|c|c|c|}
\hline \multirow{3}{*}{\multicolumn{2}{|c|}{ DESCRIPTION }} & \multicolumn{9}{|c|}{$\begin{array}{c}\text { ENTS 2 } \\
\text { CWO and Vitrification }\end{array}$} \\
\hline & & \multirow{2}{*}{$\begin{array}{l}\text { FAC. } \\
\text { CAT. }\end{array}$} & \multirow[b]{2}{*}{ HP } & \multicolumn{4}{|c|}{ MATLS. \& EQUIP. } & \multicolumn{3}{|c|}{ INST. COSTS } \\
\hline & & & & QT & & $\begin{array}{l}\text { Unit Cost } \\
\text { S1000's }\end{array}$ & $\begin{array}{l}\text { Amount } \\
\text { S1000's }\end{array}$ & $\begin{array}{c}\text { Unit Cost } \\
\text { S1000's }\end{array}$ & $\begin{array}{l}\text { Amount } \\
\text { S1000's }\end{array}$ & $\begin{array}{l}\text { Total } \\
\text { U.0. }\end{array}$ \\
\hline 3. & Residue \&s Sud ge Treatiment Subsystem & II & & & & & & 10, & & \\
\hline & - Feed Preparation and Size Reduction & & & & & & & & & \\
\hline & - Feed Conveyor Lift/Hoist/Bins & $E$ & & 1 & 1 & & & & & \\
\hline & - Discharge. \& Oversize Load Hopper (100 CF) & $\mathrm{E}$ & & 1 & 1 & & & & & \\
\hline & - Manipulate Crane $10 \mathrm{TN}$ & $E$ & 15 & 1 & 1 & & & & & \\
\hline & - $\quad$ Shield Window & $E$ & & 1 & 1 & & & & & \\
\hline & - Airlock & $\mathrm{E}$ & & 2 & 2 & & & & & \\
\hline & - Closed Circuit TV & $\bar{E}$ & & 10 & 10 & & & & & \\
\hline & - Feed Blender & $E$ & & 1 & 1 & & & & & \\
\hline & - Dewatering System & $E$ & & 1 & 1 & & & & & \\
\hline & THERMAL DESORPTION & & & & & & & & & \\
\hline & - High Vacuum Dryer & & & 1 & 1 & & & & & \\
\hline & - Nitrogen gas storage and feed tank & $\mathrm{E}$ & & 1 & 1 & & & & & \\
\hline & - Vacuum Pump & $E$ & & 1 & 1 & & & & & \\
\hline & - Filter & $E$ & & 1 & 1 & & & & . & \\
\hline & - Hot Oil System & & & & & & & & & \\
\hline & - Oil Storage and Feed Tank & E & & 1 & 1 & & & & & \\
\hline & - Oil Heater & E & & 1 & 1 & & & & & \\
\hline & - Fuel Storage and Feed Tank & E & & 1 & 1 & & & & & \\
\hline & - Condensers & & & & & & & & & \\
\hline & - HEPA Filter for particulates & $\mathrm{E}$ & & 1 & 1 & & & & & \\
\hline & - Induced Air Fan & $\mathrm{E}$ & & 1 & 1 & & & & & \\
\hline & - Primary Condensers (Air Cooler) & $E$ & & 1 & 1 & & & & & \\
\hline & - Secondary Condensers (Water Chiller) & $\mathrm{E}$ & & 2 & 2 & & & & & \\
\hline & - Total per Package Above & $E$ & & Lot & 1 & & & & & \\
\hline & - Mercury Removal System & & & & & & & & & \\
\hline & - Mercury Precipitation Tank & $\mathbf{E}$ & & 1 & 1 & & & & & \\
\hline & - Iodine Recovery Tank & $\mathrm{E}$ & & 1 & 1 & & & & & \\
\hline & - KU/2 Storage and Feed & $\mathrm{E}$ & & 1 & 1 & & & & & \\
\hline & - Metal Precipitation Tank & $E$ & & 1 & 1 & & & & & \\
\hline & - Mercury Reduction Tank & $\mathrm{E}$ & & 1 & 1 & & & & & \\
\hline & - Solids Wash Tank & $E$ & & 1 & 1 & & & & & \\
\hline & - Water Holding Tank & $E$ & & 1 & 1 & & & & & \\
\hline & - Acid Holding and Feed Tank & $E$ & & 1 & 1. & & & & & \\
\hline & $-\mathrm{H}_{2} \mathrm{O} 2$ Holding and Feed Tank & $E$ & & 1 & 1 & & & & & \\
\hline & - Lime Holding Tank & $E$ & & 1 & 1 & & & & & \\
\hline & - Rinse Tank & $\mathrm{E}$ & & 1 & 1 & & & & & \\
\hline & - Filter & $E$ & & 1 & 1 & & & & & \\
\hline & - Pump & E & & 10 & 10 & & & & & \\
\hline & - Allowance for Mechanical/Control/Instrumentation & E & & Lot & 1 & & & & & \\
\hline & - Allowance For Electrical/Control & $\mathrm{E}$ & & Lot & 1 & & & & & \\
\hline & F Calibration, Testing \& Startup & $E$ & & Lot & 1 & & & & & \\
\hline & & & & & & & & & & \\
\hline & Total Residue \& Sludge Treatment Subsystem & & & $\$$ & & & & & & \\
\hline 6 & Soft Debris I reatment Subsssten $/$ & 算 & & $\sqrt{18}$ & $\%$ & & 0 & & $\%$ & \\
\hline & - Prepare \& Feed Incoming Waste & & & & & & & & & \\
\hline & - Feed Conveyor Lift/Hoist/Bins & E & & 1 & 1 & 50 & 50 & 8 & 8 & 58 \\
\hline & - Discharge and Loading Hoppers & E & & 1 & 1 & 45 & 45 & 8 & 8 & 53 \\
\hline & Feed Blender & $E$ & & 1 & 1 & 45 & 45 & 8 & 8 & 53 \\
\hline & - Detox Process & & & & & & & & & \\
\hline & - Reaction Vessel & $E$ & & & & & & & & \\
\hline & - Reflux Condenser & $E$ & & - & & & & & & \\
\hline & - Filter & E & & & & & & & & \\
\hline & - Solids Feeder & $E$ & & & & & & & & \\
\hline & - Solution Cooler & E & & & & & & & & \\
\hline & - Overhead Condenser & $E$ & & & & & & & & \\
\hline
\end{tabular}


Table B-2.1: Equipment Purchase and Installation Budget Estimates

Enhanced Non-Thermal Treatment System

ENTS - 2 CWO and Vitrification

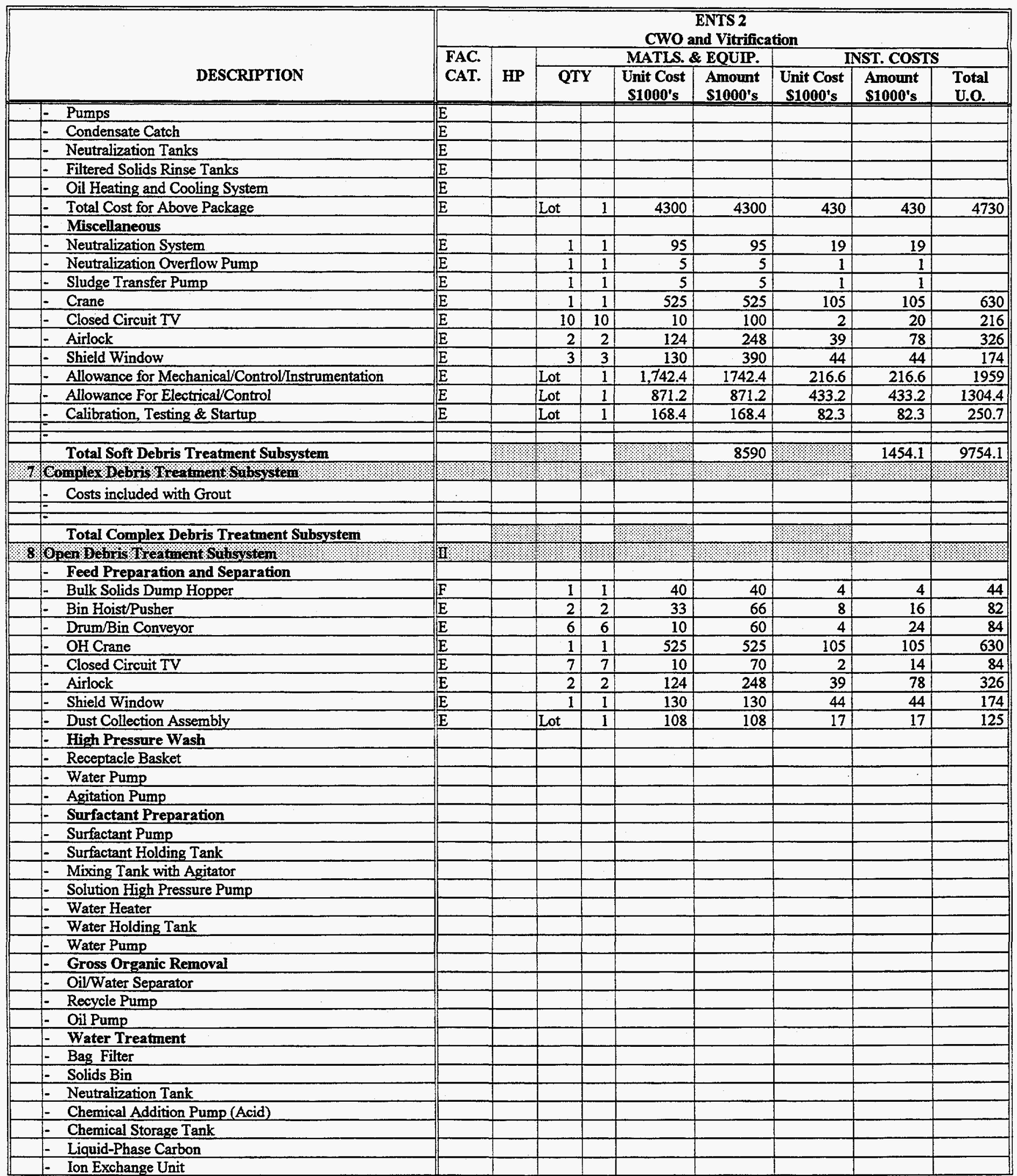


Table B-2.1: Equipment Purchase and Installation Budget Estimates

Enhanced Non-Thermal Treatment System

ENTS - 2 CWO and Vitrification

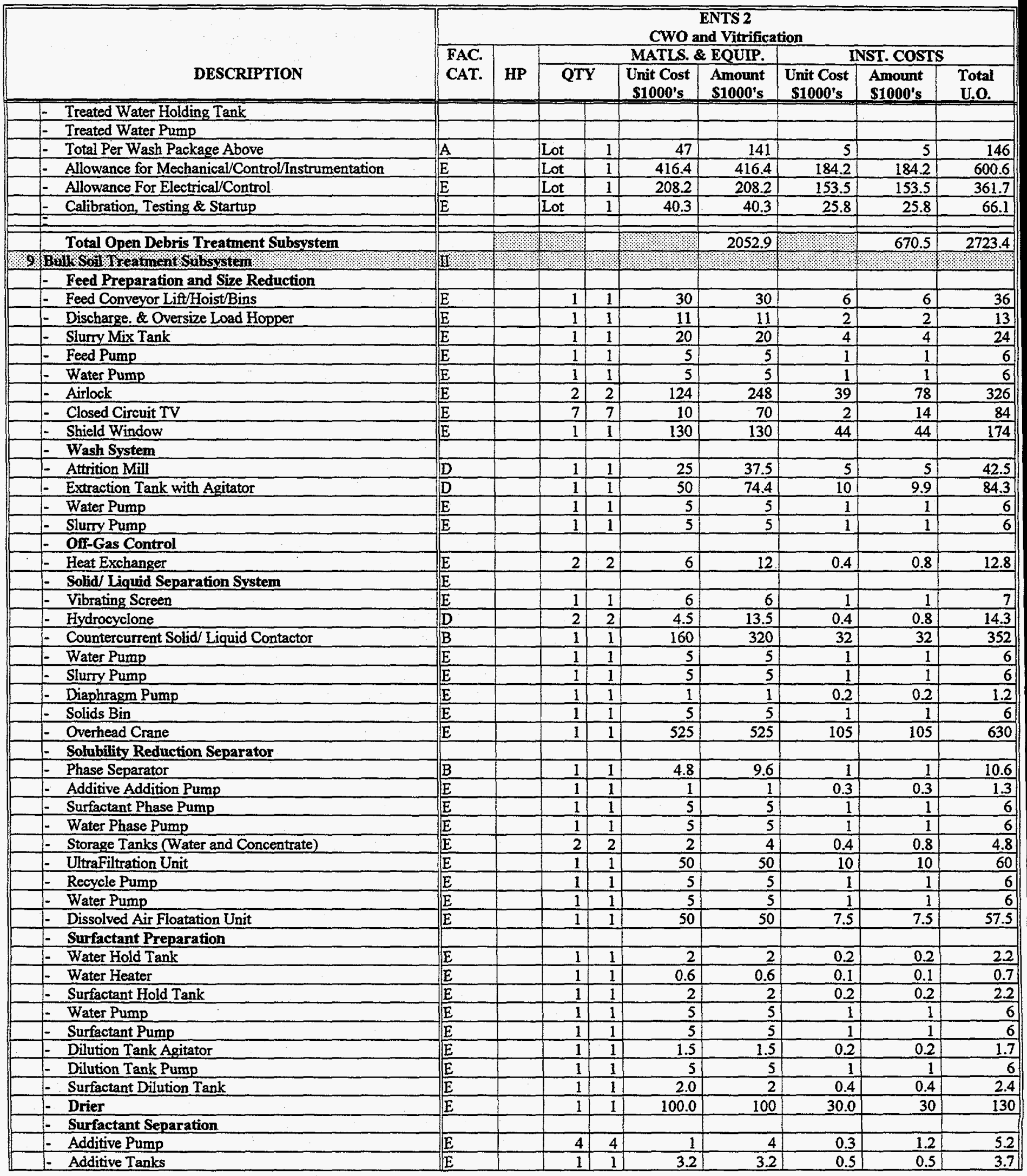


Table B-2.1: Equipment Purchase and Installation Budget Estimates

Enhanced Non-Thermal Treatment System

ENTS - 2 CWO and Vitrification

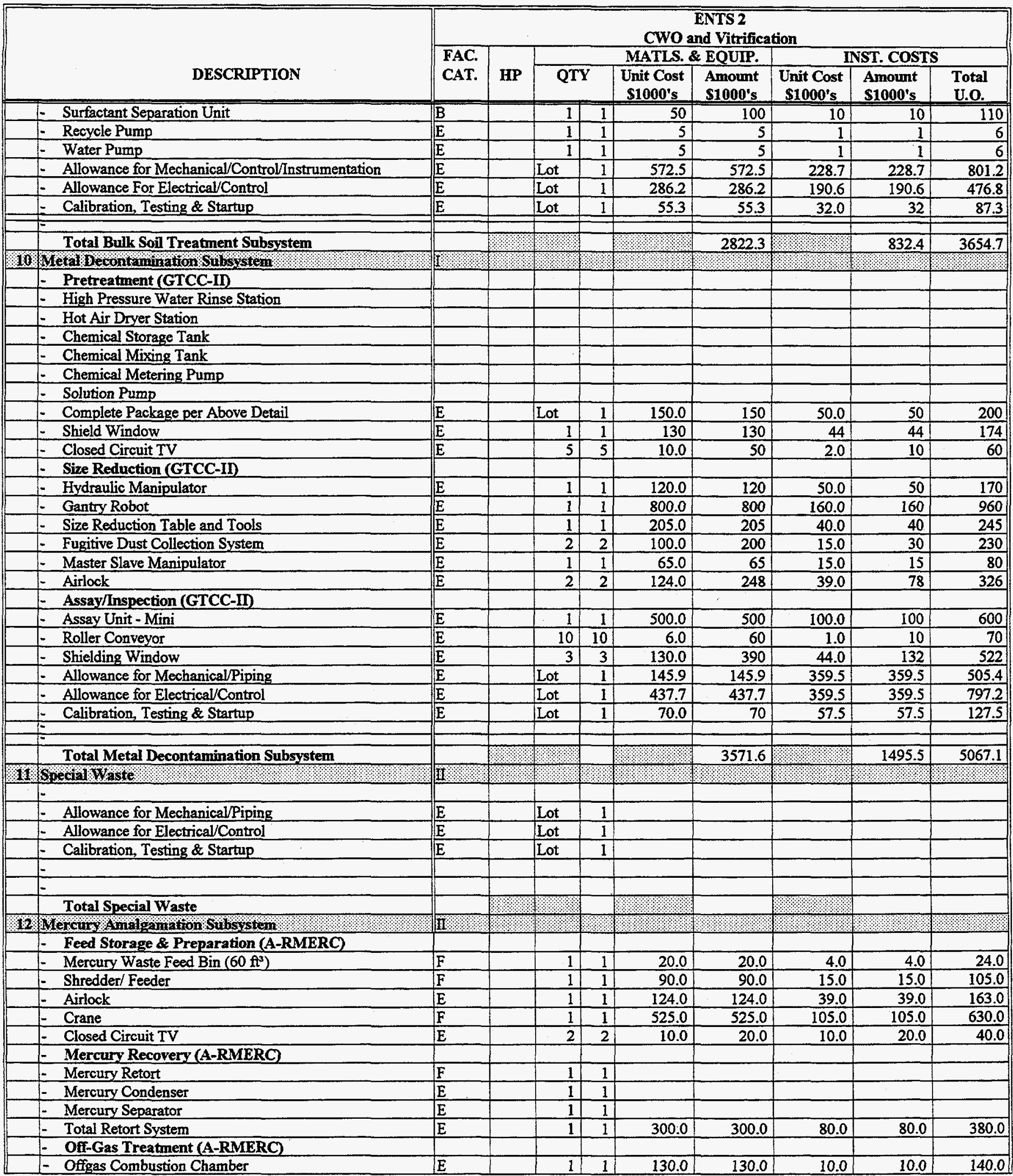


Table B-2.1: Equipment Purchase and Installation Budget Estimates

Enhanced Non-Thermal Treatment System

ENTS - 2 CWO and Vitrification

\begin{tabular}{|c|c|c|c|c|c|c|c|c|c|c|}
\hline \multirow{3}{*}{\multicolumn{2}{|c|}{ DESCRIPTION }} & \multicolumn{9}{|c|}{$\begin{array}{c}\text { ENTS 2 } \\
\text { CWO and Vitrification }\end{array}$} \\
\hline & & \multirow{2}{*}{$\begin{array}{l}\text { FAC. } \\
\text { CAT. }\end{array}$} & \multirow[b]{2}{*}{$\mathbf{H P}$} & \multicolumn{4}{|c|}{ MATLS. \& EQUIP. } & \multicolumn{3}{|c|}{ INST. COSTS } \\
\hline & & & & \multicolumn{2}{|c|}{ QTY } & $\begin{array}{l}\text { Unit Cost } \\
\$ 1000 \text { 's }\end{array}$ & $\begin{array}{l}\text { Amount } \\
\text { S1000's }\end{array}$ & $\begin{array}{c}\text { Unit Cost } \\
\$ 1000^{\prime} s\end{array}$ & $\begin{array}{l}\text { Amount } \\
\text { \$1000's }\end{array}$ & $\begin{array}{l}\text { Total } \\
\text { U.O. }\end{array}$ \\
\hline & - Quencher & $\mathrm{E}$ & & 1 & 1 & 57.0 & 57.0 & 12.0 & 12.0 & 69.0 \\
\hline & - Dry Gas Filter & E & & 1 & 1 & 160.0 & 160.0 & 25.0 & 25.0 & 185.0 \\
\hline & - Carbon Adsorbers & E & & 2 & 2 & 10.0 & 20.0 & 1.0 & 2.0 & 22.0 \\
\hline & - HEPA Filters & E & & 1 & 1 & 52.0 & 52.0 & 7.0 & 7.0 & 59.0 \\
\hline & $=$ Vacuum Pump & $E$ & & 1 & 1 & 15.0 & 15.0 & 3.0 & 3.0 & 18.0 \\
\hline & - ID Blower & $\mathrm{E}$ & & 1 & 1 & 15.0 & 15.0 & 3.0 & 3.0 & 18.0 \\
\hline & - Stack $\left(3^{\prime} ø \times 30^{\prime}\right)$ & $E$ & & 1 & 1 & 20.0 & 20.0 & 5.0 & 5.0 & 25.0 \\
\hline & - Stack Monitor & E & & Lot & 1 & 350.0 & 350.0 & 140.0 & 140.0 & 490.0 \\
\hline & - Mercury Amalgamation \& Packaging (A-RMERC) & & & & & & & & & \\
\hline & - Liquid Mercury Storage Tank & E & & 1 & 1 & 7.0 & 7.0 & 1.0 & 1.0 & 8.0 \\
\hline & - Liquid Mercury Pump & $E$ & & 1 & 1 & 5.0 & 5.0 & 1.0 & 1.0 & 6.0 \\
\hline & - Copper Powder Bin $\left(1 \mathrm{fl}^{3}\right)$ & E & & 1 & 1 & 5.0 & 5.0 & 0.6 & 0.6 & 5.6 \\
\hline & - Steel Shot Bin $\left(0.5 \mathrm{ft}^{3}\right)$ & E & & 1 & 1 & 5.0 & 5.0 & 0.6 & 0.6 & 5.6 \\
\hline & - Nitric Acid Tank (1 GAL) & $E$ & & 1 & 1 & 5.0 & 5.0 & 0.5 & 0.5 & 5.5 \\
\hline & - Nitric Acid Pump ( $1 \mathrm{gal} / \mathrm{hr}$ ) & E & & 1 & 1 & 5.0 & 5.0 & 1.0 & 1.0 & 6.0 \\
\hline & - Amalgam Mixer (5 GAL) & $F$ & & 1 & 1 & 26.0 & 26.0 & 5.0 & 5.0 & 31.0 \\
\hline & - Amalgam packager & $F$ & & 1 & 1 & 2.0 & 2.0 & 0.4 & 0.4 & 2.4 \\
\hline & - Allowance for Mechanical/Piping & $E$ & & Lot & 1 & 587.4 & 587.4 & 288.1 & 288.1 & 875.5 \\
\hline & - Allowance for Electrical/Control & E & & Lot & 1 & 293.7 & 293.7 & 240.1 & 240.1 & 533.8 \\
\hline & - Calibration, Testing \& Startup & E & & Lot & 1 & 56.8 & 56.8 & 40.3 & 40.3 & 97.1 \\
\hline & - & & & & & & & & & \\
\hline & Total Mercury Amalgamation Subsystem & & & & & & 2895.9 & & 1048.6 & 3944.5 \\
\hline 13 & LeallRecove w Sub w stem & II & & & & & 1 & 7 & & 18 \\
\hline & - Battery Disassembly (A-PBRCR) & & & & & & & & & \\
\hline & - Roller Conveyors (ft) & $\mathrm{F}$ & & 50 & 50 & 4.0 & 200.0 & 1.0 & 50.0 & 250.0 \\
\hline & - Shredder & $\mathbf{F}$ & & 1 & 1 & 42.0 & 42.0 & 7.0 & 7.0 & 49.0 \\
\hline & - Hydraulic Drum Dumper & $\mathbf{F}$ & & 1 & 1 & 3.0 & 3.0 & 1.0 & 1.0 & 4.0 \\
\hline & - Hydraulic Drum Dumper w/ Spout & $F$ & & 1 & 1 & 10.0 & 10.0 & 2.0 & 2.0 & 12.0 \\
\hline & - Acid Resist Sorting Table & $E$ & & 1 & 1 & 5.0 & 5.0 & 1.0 & 1.0 & 6.0 \\
\hline & - Acid Drain Tank & E & & 1 & 1 & 5.0 & 5.0 & 1.0 & 1.0 & 6.0 \\
\hline & - Battery Cutting Saw & F & & 1 & 1 & 2.0 & 2.0 & 1.0 & 1.0 & 3.0 \\
\hline & - Airlock & $\mathrm{E}$ & & 1 & 1 & 124.0 & 124.0 & 39.0 & 39.0 & 163.0 \\
\hline & - Crane & $E$ & & 1 & 1 & 525.0 & 525.0 & 105.0 & 105.0 & 630.0 \\
\hline & - Closed Circuit TV & E & & 2 & 2 & 10.0 & 20.0 & 2.0 & 4.0 & 24.0 \\
\hline & Decontamination (A-PBRCR) & & & & & & & & & \\
\hline & Drum Lid Detacher & $\mathrm{F}$ & & 1 & $\mathrm{I}$ & 4.0 & 4.0 & 1.0 & $1: 0$ & 5.0 \\
\hline & - Roller Conveyors (Straight) & F & & 12 & 12 & 5.0 & 60.0 & 1.0 & 12.0 & 72.0 \\
\hline & - Decon Booth & F & & 1 & 1 & 175.0 & 175.0 & 25.0 & 25.0 & 200.0 \\
\hline & - Hydraulic Drum Dumper & $\vec{F}$ & & 1 & 1 & 4.0 & 4.0 & 2.0 & 2.0 & 6.0 \\
\hline & - Sorting Table (1 Hole) & $\mathrm{E}$ & & 2 & 2 & 4.0 & 8.0 & 1.0 & 2.0 & 10.0 \\
\hline & - Sorting Table (4 Holes) & E & & 1 & 1 & 6.0 & 6.0 & 1.0 & 1.0 & 7.0 \\
\hline & - Rolling Conveyor (L-Shaped) & $\mathrm{F}$ & & 1 & 1 & 5.0 & 5.0 & 2.0 & 2.0 & 7.0 \\
\hline & - Drum Pusher & $F$ & & 1 & 1 & 1.0 & 1.0 & 1.0 & 1.0 & 2.0 \\
\hline & - Aqueous Waste Tank & 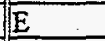 & & 1 & 1 & 5.0 & 5.0 & 2.0 & 2.0 & 7.0 \\
\hline & - Aqueous Waste Pump & $\mathrm{E}$ & & 1 & 1 & 3.0 & 3.0 & 2.0 & 2.0 & 5.0 \\
\hline & - Lead Melter (A-PBRCR) & & & & & & & & & \\
\hline & - Melting Furnace w/ Hood & $\mathrm{F}$ & & 1 & $\mathrm{l}$ & 100.0 & 100.0 & 30.0 & 30.0 & 130.0 \\
\hline & - Crucibles & E & & 1 & 1 & 1.0 & 1.0 & 0.2 & 0.2 & 1.2 \\
\hline & - Sodium Carbonate Tank & $\mathrm{E}$ & & 1 & 1 & 5.0 & 5.0 & 0.2 & 0.2 & 5.2 \\
\hline & - Sodium Carbonate Pump & E & & $\frac{2}{1}$ & $\frac{1}{1}$ & 5.0 & 5.0 & 0.2 & 0.2 & 5.2 \\
\hline & - Stirrer & $E$ & & 1 & 1 & 15.0 & 15.0 & 2.5 & 2.5 & 17.5 \\
\hline & - Casting (A-PBRCR) & & & & & & & & & \\
\hline & - Lead Casting & $F$ & & 1 & 1 & 88.0 & 88.0 & 10.0 & 10.0 & 98.0 \\
\hline & - Cast Lead Cooling Bin & $F$ & & 4 & 4 & 5.0 & 20.0 & 0.3 & 1.2 & 21.2 \\
\hline & - Assay/Drumming (A-PBRCR) & & & & & & & & & \\
\hline & - Drum Pusher & $F$ & & 1 & 1 & 10.0 & 10.0 & 0.2 & 0.2 & 10.2 \\
\hline & - Lid Attacher & $\mathrm{F}$ & & 1 & 1 & 2.0 & 2.0 & 0.3 & 0.3 & 2.3 \\
\hline
\end{tabular}


Table B-2.1: Equipment Purchase and Installation Budget Estimates

Enhanced Non-Thermal Treatment System

\section{ENTS - 2 CWO and Vitrification}

\begin{tabular}{|c|c|c|c|c|c|c|c|c|c|c|}
\hline \multirow{3}{*}{\multicolumn{2}{|c|}{ DESCRIPTION }} & \multicolumn{9}{|c|}{$\begin{array}{c}\text { ENTS 2 } \\
\text { CWO and Vitrification }\end{array}$} \\
\hline & & \multirow{2}{*}{$\begin{array}{l}\text { FAC. } \\
\text { CAT. }\end{array}$} & \multirow[b]{2}{*}{ HP } & \multicolumn{4}{|c|}{ MATLS. \& EQUIP. } & \multicolumn{3}{|c|}{ INST. COSTS } \\
\hline & & & & \multicolumn{2}{|c|}{ QTY } & $\begin{array}{l}\text { Unit Cost } \\
\$ 1000 \text { 's }\end{array}$ & $\begin{array}{l}\text { Amount } \\
\text { S1000's }\end{array}$ & $\begin{array}{l}\text { Unit Cost } \\
\text { S1000's }\end{array}$ & $\begin{array}{l}\text { Amount } \\
\text { S1000's }\end{array}$ & $\begin{array}{l}\text { Total } \\
\text { U.0. }\end{array}$ \\
\hline & - Assay Station & $\mathrm{F}$ & & 1 & 1 & 500.0 & 500.0 & 3.0 & 3.0 & 503.0 \\
\hline & Drum Washer & E & & 1 & $\frac{1}{1}$ & 70.0 & 70.0 & 10.0 & 10.0 & 80.0 \\
\hline & - Miscellaneous Equipment & & & & & & & & & \\
\hline & - Transfer Cart (CA) & $E$ & & 1 & 1 & 100.0 & 100.0 & 20.0 & 20.0 & 120.0 \\
\hline & - Air Pollution Control & & & & & & & & & \\
\hline & - Quencher System & $E$ & & 1 & 1 & 100.0 & 100.0 & 20.0 & 20.0 & 120.0 \\
\hline & - Baghouse & E & & 1 & 1 & 30.0 & 30.0 & 6.0 & 6.0 & 36.0 \\
\hline & - Activate Carbon & $\mathbf{E}$ & & 1 & 1 & 50.0 & 50.0 & 10.0 & 10.0 & 60.0 \\
\hline & - HEPA Filter & $\mathrm{E}$ & & 1 & 1 & 50.0 & 50.0 & 10.0 & 10.0 & 60.0 \\
\hline & - Stack & E & & 1 & 1 & 20.0 & 20.0 & 4.0 & 4.0 & 24.0 \\
\hline & - Stack Monitor (Radioactive) & E & & Lot & 1 & 200.0 & 200.0 & 80.0 & 80.0 & 280.0 \\
\hline & - Stack Monitor (Non-Radioactive) & E & & Lot & 1 & 150.0 & 150.0 & 60.0 & 60.0 & 210.0 \\
\hline & - Allowance for Mechanical/Piping & $\mathrm{E}$ & & Lot & 1 & 816.9 & 816.9 & 317.3 & 317.3 & 1134.2 \\
\hline & - Allowance for Electrical/Control & E & & Lot & 1 & 408.5 & 408.5 & 264.4 & 264.4 & 672.9 \\
\hline & - Calibration Testing \& Startup & $E$ & & Lot & 1 & 79.0 & 79 & 44.4 & 44.4 & 123.4 \\
\hline & 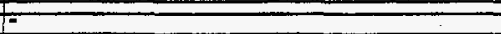 & & & & & & & & & \\
\hline & Total Lead Recovery Subsystem & & & & & & 4027.4 & & 1154.9 & 5182.3 \\
\hline 14 & Ain Pollution control Subs stem & mis & & \% & + & \% & ২৮? & \% & $1 \%$ & $\%$ \\
\hline & - Particulate Filter & & & & & & & & & \\
\hline & - Baghouse Assembly & $E$ & & 1 & 1 & 240.0 & 240 & 48.0 & 48 & 288 \\
\hline & - Prefilter & $\bar{E}$ & & 2 & 2 & 26.0 & 52 & 12.0 & 24 & 76 \\
\hline & - Inter Connection Ducts. & $E$ & & Lot & $I$ & 2.0 & 2 & 10.0 & 10 & 12 \\
\hline & - Corona Reactor & & & & & & & & & \\
\hline & - Reactor & & & & & & & & & \\
\hline & - Skid \& Instrumentation & & & & & & & & & \\
\hline & - Power Supply & & & & & & & & & \\
\hline & - Cost per Package Above & $E$ & & Lot & 1 & 73.0 & 73 & 7.0 & 7 & 80 \\
\hline & - Acid Gas Scrubber & & & & & & & & & \\
\hline & - Packing Tower & $E$ & & 1 & 1 & 45.0 & 45 & 13.0 & 13 & 58 \\
\hline & - Induced Fan & $E$ & & 2 & 2 & 4.5 & 9 & 0.5 & 1 & 10 \\
\hline & - Recycle Pump & $E$ & & 2 & 2 & 1.8 & 3.6 & 0.2 & 0.4 & 4 \\
\hline & - Sludge Discharge Pump & $\bar{E}$ & & 2 & 2 & 2.7 & 5.4 & 0.5 & 1 & $\overline{6.4}$ \\
\hline & - Caustic Feed Pump & $E$ & & 2 & 2 & 0.9 & 1.8 & 0.2 & 0.4 & 2.2 \\
\hline & - NaOH Transfer Pump & $\bar{E}$ & & 2 & 2 & 0.9 & 1.8 & 0.2 & 0.4 & 2.2 \\
\hline & - Caustic Storage Tank & $E$ & & 1 & 1 & 0.9 & 0.9 & 0.2 & 0.2 & 1.1 \\
\hline & - Slurry Pump & $\bar{E}$ & & 2 & 2 & 2.7 & 5.4 & 0.5 & 1 & 6.4 \\
\hline & - Condensate Tank & $\mathrm{E}$ & & 1 & 1 & 0.9 & 0.9 & 0.2 & 0.2 & 1.1 \\
\hline & - $\mathrm{NaOH}$ Day Tank & $E$ & & 1 & 1 & 0.9 & 0.9 & 0.2 & 0.2 & 1.1 \\
\hline & - Scrubber Solution Tank & $E$ & & 1 & 1 & 0.9 & 0.9 & 0.2 & 0.2 & 1.1 \\
\hline & - Evaporator System & $E$ & & 1 & 1 & 54.0 & 54 & 16.2 & 16.2 & 70.2 \\
\hline & - Hydrosonic Scrubber & $E$ & & 1 & 1 & 27.0 & 27 & 5.4 & 5.4 & 32.4 \\
\hline & - Liquid/Solid Separator & $\bar{E}$ & & 1 & 1 & 18.0 & 18 & 3.6 & 3.6 & 21.6 \\
\hline & - Mist Eliminator & E & & 1 & 1 & 1.6 & 1.6 & 0.4 & 0.4 & 2 \\
\hline & - Heat Exchanger & $E$ & & 2 & 2 & 5.4 & 10.8 & 1.8 & 3.6 & 14.4 \\
\hline & - Carbon Adsorption & & & & & & & & & \\
\hline & - Carbon Filter (GAC) & $F$ & & 2 & 2 & 9.0 & 18 & 1.8 & 3.6 & 21.6 \\
\hline & - Carbon Filter (Sublimated GAC) & $\bar{F}$ & & 2 & 2 & 9.0 & 18 & 1.8 & 3.6 & 21.6 \\
\hline
\end{tabular}


Table B-2.1: Equipment Purchase and Installation Budget Estimates

Enhanced Non-Thermal Treatment System

ENTS - 2 CWO and Vitrification

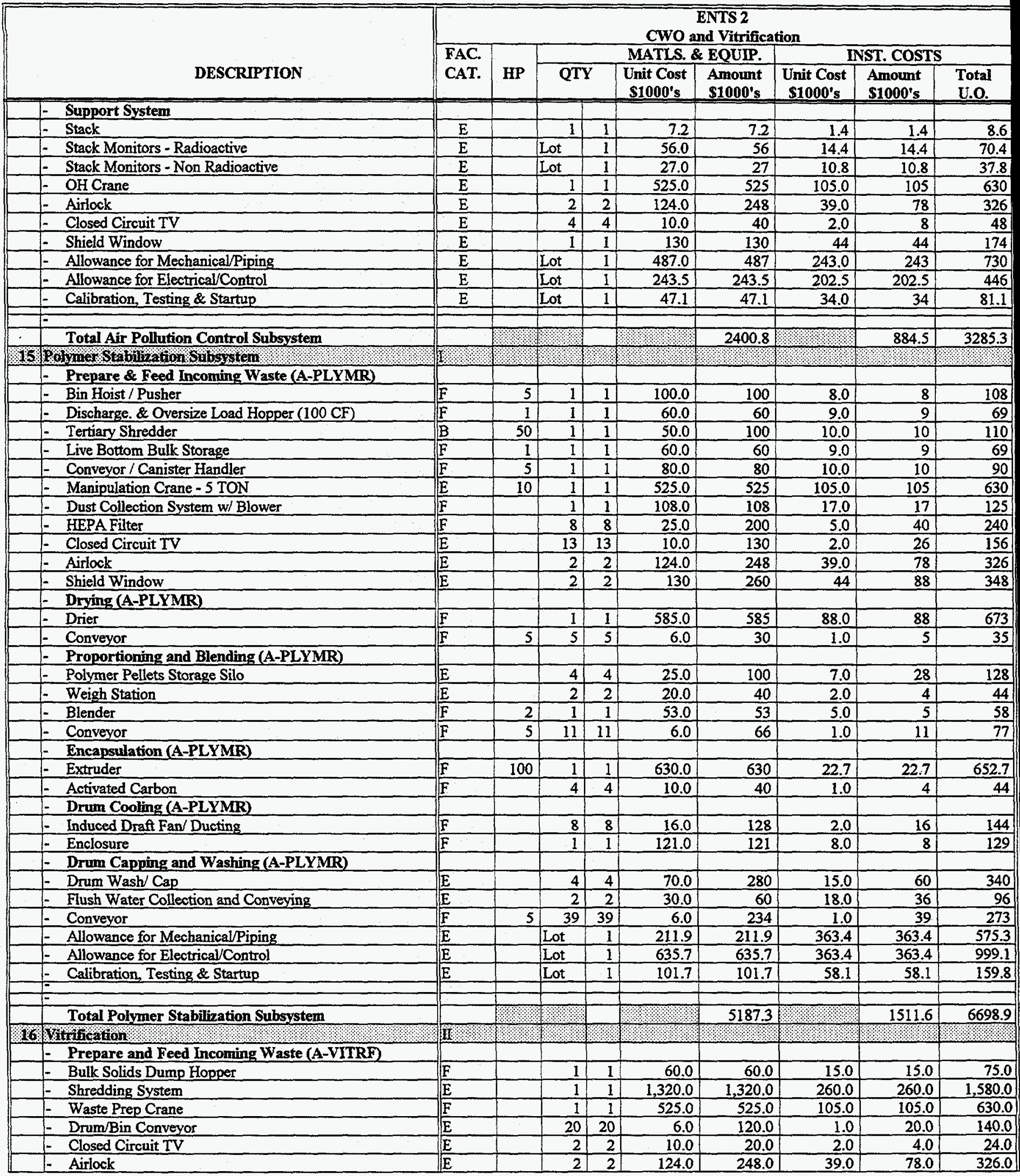


Table B-2.1: Equipment Purchase and Installation Budget Estimates

Enhanced Non-Thermal Treatment System

ENTS - 2 CWO and Vitrification

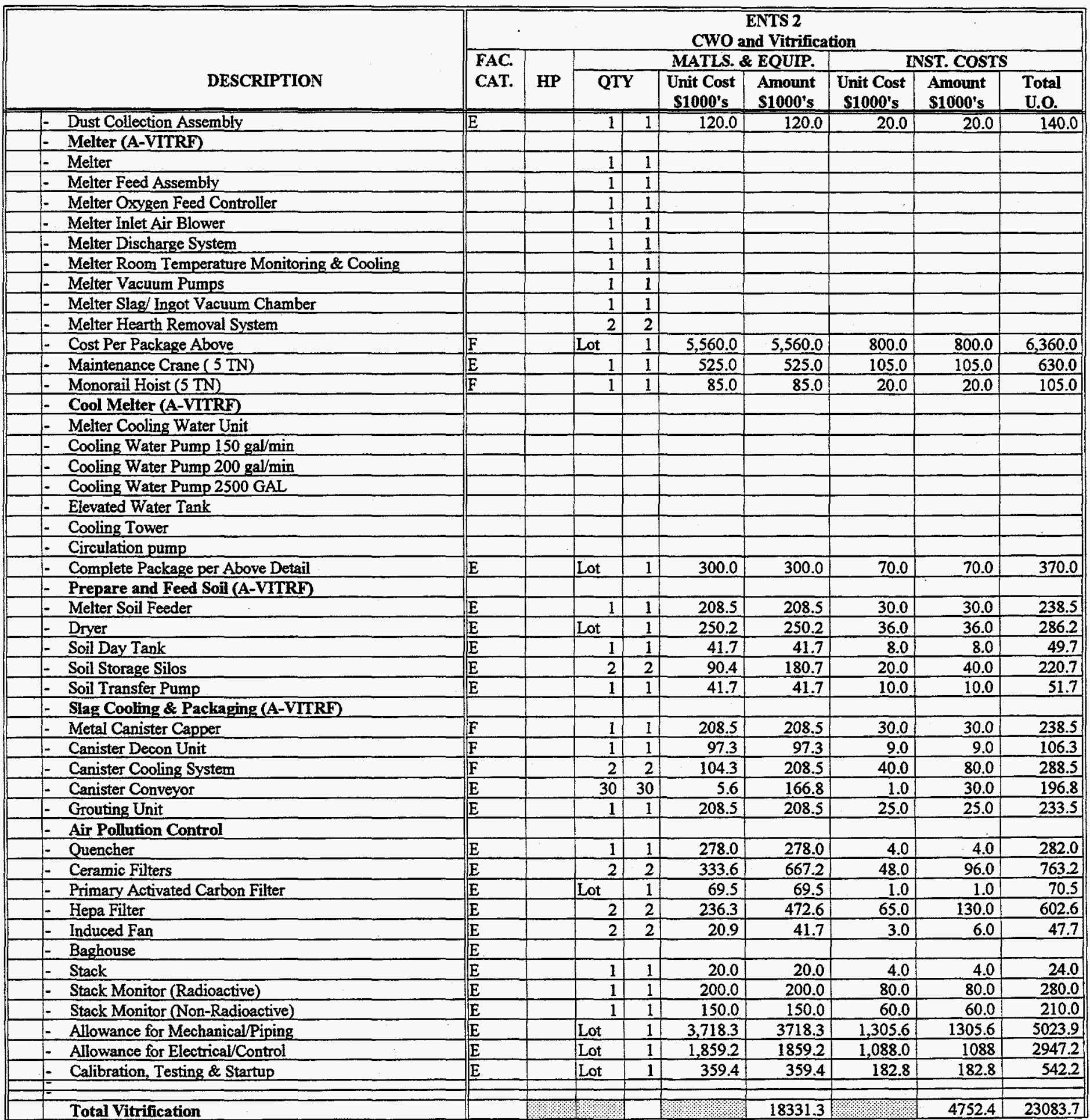


Table B-2.1: Equipment Purchase and Installation Budget Estimates

Enhanced Non-Thermal Treatment System

ENTS - 2 CWO and Vitrification

\begin{tabular}{|c|c|c|c|c|c|c|c|c|c|c|}
\hline & \multirow[b]{3}{*}{ DESCRIPTION } & \multicolumn{9}{|c|}{$\begin{array}{c}\text { ENTS 2 } \\
\text { CWO and Vitrification }\end{array}$} \\
\hline & & \multirow{2}{*}{$\begin{array}{l}\text { FAC. } \\
\text { CAT. }\end{array}$} & \multirow[b]{2}{*}{ HP } & \multicolumn{4}{|c|}{ MATLS. \& EQUIP. } & \multicolumn{3}{|c|}{ INST. COSTS } \\
\hline & & & & \multicolumn{2}{|c|}{ QTY } & $\begin{array}{c}\text { Unit Cost } \\
\$ 1000 \text { 's }\end{array}$ & $\begin{array}{l}\text { Amount } \\
\text { \$1000's }\end{array}$ & $\begin{array}{l}\text { Unit Cost } \\
\text { \$1000's }\end{array}$ & $\begin{array}{l}\text { Amount } \\
\text { S1000's }\end{array}$ & $\begin{array}{l}\text { Total } \\
\text { U. } 0\end{array}$ \\
\hline 17 & Cerfification \& Shipping Sabsystem & 1 & & & \% & 1313 & & & & \\
\hline & - Incoming Material Storage (B-CSHIP) & & & & & & & & & \\
\hline & - Overhead Bridge Crane $20 \mathrm{TN}$ & $E$ & & 1 & 1 & 625.0 & 625 & 125.0 & 125 & 750 \\
\hline & - Forklift $10 \mathrm{TN}$ & E & & 1 & 1 & 65.0 & 65 & 10.0 & 10 & 75 \\
\hline & - Inspect \& Assay (B-CSHIP) & & & & & & & & & \\
\hline & - Transfer Cart 2 TN & E & & & & & & & & \\
\hline & - Real Time Radiography (ND) & & & & & & & & & \\
\hline & - Passive Active Neutron (ND) & & & & & & & & & \\
\hline & - Segmented Gamma Spectroscopy (ND) & & & & & & & & & \\
\hline & - Complete Package per Above & $E$ & & 1 & 1 & $1,575.0$ & 1575 & 150.0 & 150 & 1725 \\
\hline & - Self Guided Vehicle (ND) & $E$ & & 1 & 1 & 425.0 & 425 & 850.0 & 850 & 1275 \\
\hline & - Truck Loading (B-CSHIP) & & & & & & & & & \\
\hline & Forklift $10 \mathrm{TN}$ & $\mathbf{E}$ & & 10 & 10 & 65.0 & 650 & 10.0 & 100 & 750 \\
\hline & - Roll-up Door & E & & 2 & 2 & 20.0 & 40 & 2.0 & 4 & 44 \\
\hline & - Bridge Crane & E & & 1 & 1 & 525.0 & 525 & 105.0 & 105 & 630 \\
\hline & - Roller Conveyor (CA) & $E$ & & 20 & 20 & 2.0 & 40 & 1.0 & 20 & 60 \\
\hline & - Allowance for Mechanical/Piping & $E$ & & Lot & 1 & 197.3 & 197.3 & 682.0 & 682 & 879.3 \\
\hline & - Allowance for Electrical/Control & $E$ & & Lot & 1 & 591.8 & 591.8 & 682.0 & 682 & 1273.8 \\
\hline & $=$ Calibration, Testing \& Startup & E & & Lot & 1 & 94.7 & 94.7 & 109.1 & 109.1 & 203.8 \\
\hline & Total Certification \& Shipping Subsystem & & & & & & 4828.8 & & 2837.1 & 7665.9 \\
\hline 18 & Support Subsystem & WI & & & 1 & $\frac{1}{1 \%}$ & 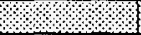 & 1 & & $1 \%$ \\
\hline & - Electrical Distribution and MCC & & & & & & & & & \\
\hline & - Control Panel - FT & $E$ & & 110 & 110 & 10.0 & 1100 & 2.0 & 220 & 1320 \\
\hline & - Emergency Power Generator & E & & 1 & 1 & 500.0 & 500 & 100.0 & 100 & 600 \\
\hline & - Main Control Panel & $E$ & & 20 & 20 & 10.0 & 200 & 2.0 & 40 & 240 \\
\hline & Motor Control Center & E & & 100 & 100 & 1.5 & 150 & 0.5 & 50 & 200 \\
\hline & - Stand by Emergency Power & E & & 1 & 1 & 230.0 & 230 & 80.0 & 80 & 310 \\
\hline & - Utilities and Mechanical & & & & & & & & & \\
\hline & - Breathing Air Package & $E$ & & Lot & 1 & 70.0 & 70 & 30.0 & 30 & 100 \\
\hline & - Compressed Air Package & E & & Lot & 1 & 100.0 & 100 & 25.0 & 25 & 125 \\
\hline & - Instrument Air & $E$ & & 1 & 1 & 70.0 & 70 & 30.0 & 30 & 100 \\
\hline & - Instrument Air Package & $E$ & & Lot & 1 & 90.0 & 90 & 25.0 & 25 & 115 \\
\hline & - Plant Steam/Boiler & E & & 1 & 1 & 75.0 & 75 & 25.0 & 25 & 100 \\
\hline & - Service Water & $E$ & & 1 & 1 & 130.0 & 130 & 40.0 & 40 & 170 \\
\hline & - Sump Pump & $E$ & 3 & 10 & 10 & 5.0 & 50 & 2.0 & 20 & 70 \\
\hline & - Cooling Tower & $\underline{E}$ & & 1 & 1 & 200.0 & 200 & 10.0 & 10 & 210 \\
\hline & - Cooling Water Pumps & $\mathrm{E}$ & 3 & 2 & 2 & 15.0 & 30 & 3.0 & 6 & 36 \\
\hline & - Heating, Ventilation \& Exhaust & & & & & & & & & \\
\hline & - Included with Building Cost & & & & & & & & & \\
\hline & - Radiation Monitoring & & & & & & & & & \\
\hline & - Air Monitors & E & & 44 & 44 & 10.0 & 440 & 2.0 & 88 & 528 \\
\hline & - Area Monitoring & E & & 44 & 44 & 4.5 & 198 & 1.0 & 44 & 242 \\
\hline & - Personnel Monitoring Portals & $E$ & & 6 & 6 & 35.0 & 210 & 5.0 & 30 & 240 \\
\hline & - Portal Monitors & $\mathrm{E}$ & & 6 & 6 & 35.0 & 210 & 5.0 & 30 & 240 \\
\hline & - Allowance for Mechanical/Piping & $\mathrm{E}$ & & Lot & 1 & 40.5 & 40.5 & 8.9 & 8.9 & 49.4 \\
\hline & - Allowance for Electrical/Control & $E$ & & Lot & 1 & 608.0 & 608 & 134.0 & 134 & 742 \\
\hline & - Calibration, Testing \& Startup & $E$ & & Lot & 1 & 94.0 & 94 & 20.7 & 20.7 & 114.7 \\
\hline & & & & & & & & & & \\
\hline & Total Support Subsystem & & & & & 1 & 4795.5 & & 1056.6 & 5852.1 \\
\hline & $101 \%$ & & & 12 & & $2+$ & & & \& & \\
\hline
\end{tabular}


Table B-2.1: Equipment Purchase and Installation Budget Estimates

Enhanced Non-Thermal Treatment System

ENTS - 2 CWO and Vitrification

\begin{tabular}{|c|c|c|c|c|c|c|c|c|c|c|}
\hline \multirow{3}{*}{\multicolumn{2}{|c|}{ DESCRIPTION }} & \multicolumn{9}{|c|}{$\begin{array}{c}\text { ENTS 2 } \\
\text { CWO and Vitrification }\end{array}$} \\
\hline & & \multirow{2}{*}{$\begin{array}{l}\text { FAC. } \\
\text { CAT. }\end{array}$} & \multirow[b]{2}{*}{ HP } & \multicolumn{4}{|c|}{ MATLS. \& EQUIP. } & \multicolumn{3}{|c|}{ INST. COSTS } \\
\hline & & & & \multicolumn{2}{|c|}{ QTY } & $\begin{array}{l}\text { Unit Cost } \\
\$ 1000 \text { 's }\end{array}$ & $\begin{array}{l}\text { Amount } \\
\text { S1000's }\end{array}$ & $\begin{array}{l}\text { Unit Cost } \\
\text { S1000's }\end{array}$ & $\begin{array}{l}\text { Amount } \\
\text { \$1000's }\end{array}$ & $\begin{array}{l}\text { Total } \\
\text { U.O. }\end{array}$ \\
\hline 199 & Metal Melting Subsustem & H & & & & & & & \% & \\
\hline & - Prepare and Feed Incoming Waste (A-MMELI & & & & & & & & & \\
\hline & - Bulk Solids Dump Hopper & F & & 1 & 1 & 45.0 & 45 & 10.0 & 10.0 & 55.0 \\
\hline & - Gantry Robot & F & & 1 & 1 & 525.0 & 525 & 105.0 & 105.0 & 630.0 \\
\hline & - Master Slave Manipulator & E & & 1 & 1 & 65.0 & 65 & 15.0 & 15.0 & 80.0 \\
\hline & - Size Reduction Table with Tools & $\mathrm{E}$ & & 1 & 1 & 120.0 & 120 & 24.0 & 24.0 & 144.0 \\
\hline & - Drum/Bin Conveyor & $F$ & & 20 & 20 & 6.0 & 120 & 1.0 & 20.0 & 140.0 \\
\hline & - Closed Circuit TV & $\mathrm{E}$ & & 2 & 2 & 10.0 & 20 & 4.0 & 8.0 & 28.0 \\
\hline & - Airlock & $\mathrm{E}$ & & 2 & 2 & 124.0 & 248 & 39.0 & 78.0 & 326.0 \\
\hline & - Dust Collection Assembly & E & & 1 & 1 & 100.0 & 100 & 15.0 & 15.0 & 115.0 \\
\hline & - Melter (A-MMELT) & & & & & & & & & \\
\hline & - Melter & & & & & & & & & \\
\hline & - Melter Feed Assembly & & & & & & & & & \\
\hline & - Melter Secondary Combustion Chamber & & & & & & & & & \\
\hline & - Melter Oxygen Feed Controller & & & & & & & & & \\
\hline & - Melter Inlet Air Blower & & & & & & & & & \\
\hline & - Melter Discharge System & & & & & & & & & \\
\hline & - Melter Room Temperature Monitoring \& Cooling & & & & & & & & & \\
\hline & - Melter Vacuum Pumps & & & & & & & & & \\
\hline & - Melter Slag/Ingot Vacuum Chamber & & & & & & & & & \\
\hline & - Melter Hearth Removal System & & & & & & & & & \\
\hline & - Cost Per Package Above & $\mathbf{F}$ & & Lot & 1 & $1,200.0$ & 1200 & 500.0 & 500.0 & $1,700.0$ \\
\hline & - Maintenance Crane & E & & 1 & 1 & 450.0 & 450 & 100.0 & 100.0 & 550.0 \\
\hline & - Monorail Hoist & $\mathbf{F}$ & & 1 & 1 & 85.0 & 85 & 20.0 & 20.0 & 105.0 \\
\hline & - Cool Melter (A-MMELT) & & & & & & & & & \\
\hline & - Melter Cooling Water Unit & & & & & & & & & \\
\hline & - Cooling Water Pump $150 \mathrm{gal} / \mathrm{min}$ & & & & & & & & & \\
\hline & - Cooling Water Pump $200 \mathrm{gal} / \mathrm{min}$ & & & & & & & & & \\
\hline & - Cooling Water Pump 2500 GAL & & & & & & & & & \\
\hline & - Elevated Water Tank & & & & & & & & & \\
\hline & - Cooling Tower & & & & & & & & & \\
\hline & - Circulation pump & $\mathbf{E}$ & & & & & & & & \\
\hline & - Complete Package per Above Detail & $\mathrm{E}$ & & Lot & 1 & 150.0 & 150 & 60.0 & 60.0 & 210.0 \\
\hline & - Metal Cooling \& Packaging (A-MMELT) & & & & & & & & & \\
\hline & - Metal Canister Capper & $\mathbf{F}$ & & 1 & 1 & 150.0 & 150 & 50.0 & 50.0 & 200.0 \\
\hline & - Canister Decon Unit & $\mathbf{F}$ & & 1 & 1 & 70.0 & 70 & 20.0 & 20.0 & 90.0 \\
\hline & - Canister Cooling System & $\mathbf{F}$ & & 1 & 1 & 200.0 & 200 & 60.0 & 60.0 & 260.0 \\
\hline & - Canister Conveyor & $F$ & & 15 & 15 & 4.0 & 60 & 1.0 & 15.0 & 75.0 \\
\hline & - Air Pollution Control System & & & & & & & & & \\
\hline & - Quench System & $E$ & & 1 & 1 & 100 & 100 & 20 & 20.0 & 120.0 \\
\hline & - Filter Baghouse & $E$ & & 1 & 1 & 30 & 30 & 6 & 6.0 & 36.0 \\
\hline & - Activated Carbon Filters & $\mathrm{E}$ & & 1 & 1 & 50 & 50 & 10 & 10.0 & 60.0 \\
\hline & - HEPA Filters & $\mathbf{E}$ & & 1 & 1 & 50 & 50 & 10 & 10.0 & 60.0 \\
\hline & - Stack & E & & 1 & 1 & 20 & 20 & 4 & 4.0 & 24.0 \\
\hline & - Stack Monitor (Radioactive) & E & & 1 & 1 & 200 & 200 & 80 & 80.0 & 280.0 \\
\hline & - Stack Monitor (Non-Radioactive) & $E$ & & 1 & 1 & 150 & 150 & 60 & 60.0 & 210.0 \\
\hline & - & $\mathrm{E}$ & & & & & & & & \\
\hline & - Allowance for Mechanical/Piping & E & & Lot & 1 & $1,262.4$ & 1262.4 & 774.0 & 774 & 2036.4 \\
\hline & - Allowance for Electrical/Control & E & & Lot & 1 & 631.2 & 631.2 & 645.0 & 645 & 1276.2 \\
\hline & - Calibration, Testing \& Startup & $E$ & & Lot & 1 & 122.0 & 122 & 108.4 & 108.4 & 230.4 \\
\hline & F- & & & & & & & & & \\
\hline & Total Metal Melting Subsystem & & & & & & 6.223 .6 & & $2,817.4$ & $9,041.0$ \\
\hline
\end{tabular}



Table B-2.2 : Development, Testing \& Evaluation Cost Estimates ENTS - 2 CWO and Vitrification

\begin{tabular}{|c|c|c|c|c|c|c|c|c|c|c|}
\hline \multirow{2}{*}{\multicolumn{2}{|c|}{ UNIT OPERATION }} & \multicolumn{9}{|c|}{$\begin{array}{c}\text { ENTS - } 2 \\
\text { CWO and Vitrification } \\
\end{array}$} \\
\hline & & $\begin{array}{c}\text { Primary } \\
\text { Paper } \\
\text { Evaluation } \\
\text { Research } \\
\text { Manpower }\end{array}$ & $\begin{array}{c}\text { Bench } \\
\text { Scale } \\
\text { Studies } \\
\text { Research } \\
\text { Manpower } \\
\end{array}$ & $\begin{array}{c}\text { Sec. } \\
\text { Paper } \\
\text { Evaluation } \\
\text { Research } \\
\text { Manpower }\end{array}$ & $\begin{array}{c}\text { Total } \\
\text { Research } \\
\text { Manpower }\end{array}$ & $\begin{array}{c}\text { Bench } \\
\text { Scale } \\
\text { Studies } \\
\text { Equipment } \\
\$ 1000 \\
\end{array}$ & \begin{tabular}{|c|} 
Bench \\
Scale \\
Studies \\
Installation \\
$\$ 1000$ \\
\end{tabular} & $\begin{array}{c}\text { Pilot } \\
\text { Plant } \\
\text { Mock-up } \\
\text { Test Demo. } \\
\text { Manpower } \\
\end{array}$ & $\begin{array}{c}\text { Pilot } \\
\text { Plant } \\
\text { Mock-up } \\
\text { Test Bldg. } \\
\$ 1000 \\
\end{array}$ & $\begin{array}{c}\text { Pilot } \\
\text { Plant } \\
\text { Mock-up } \\
\text { Test Equip. } \\
\text { S1000 } \\
\end{array}$ \\
\hline 1 & Administration Building Subsystem & & & & & & & & & \\
\hline 2 & Receiving \& Inspection Subsystem & 12 & & & 12 & & & 25 & 1,200 & 6,000 \\
\hline 3 & Aqueous Waste Treatment Subsystem & 5 & 3 & 3 & 11 & 100 & 300 & 10 & 400 & 2,000 \\
\hline 4 & Organic Destruction Subsystem & 18 & 20 & 4 & 42 & 500 & 150 & 30 & 400 & 4,000 \\
\hline 5 & Residue \& Sludge Treatment Subsystem & & & & & & & & & \\
\hline 6 & Soft Debris Treatment Subsystem & & & & & & & & & \\
\hline 7 & Complex Debris Treatment Subsystem & & & & & & & & & \\
\hline 8 & Open Debris Treatment Subsystem & 2 & 6 & 2 & 10 & 300 & 50 & 20 & 1,000 & 3,000 \\
\hline 9 & Bulk Soil Treatment Subsystem & 10 & 6 & 2 & 18 & 200 & 50 & 10 & 200 & 500 \\
\hline 10 & Metal Decontamination Subsystem & & & & & & & & & \\
\hline 11 & Special Waste & 8 & 20 & 5 & 33 & 600 & 250 & 20 & 400 & 2,000 \\
\hline 12 & Mercury Amalgamation Subsystem & 1 & 3 & & 4 & 100 & 50 & & & \\
\hline 13 & Lead Recovery Subsystem & 2 & 2 & & 4 & 100 & 30 & & & \\
\hline 14 & Air Pollution Control Subsystem & 5 & 3 & 2 & 10 & 200 & 50 & 15 & 400 & 2,000 \\
\hline 15 & Polymer Stabilization Subsystem & 2 & 8 & 4 & 14 & 300 & 50 & 15 & 400 & 2,000 \\
\hline 16 & Vitrification & 2 & 8 & 4 & 14 & 300 & 50 & 15 & 400 & 1,000 \\
\hline 17 & Certification \& Shipping Subsystem & & & & & & & & & \\
\hline 18 & Support Subsystem & 5 & & & 5 & & & & & \\
\hline 19 & Metal Melting Subsystem & & & & & & & & & \\
\hline & Unit Cost (\$/Unit) & & & & $\$ 150,000$ & & & $\$ 150,000$ & & \\
\hline & Total Cost & & & & 26,550 & 2,700 & 1,030 & 24,000 & 4,800 & 22,500 \\
\hline & & Post Totals To & Table 4-2 Item & & 1.1 & 1.2 & 1.3 & 2.1 & 2.5 .1 & 2.5 .2 \\
\hline
\end{tabular}


Table B-2.3 :Building, Equipment and Installation Cost Summary ENTS - 2 CWO and Vitrification

\begin{tabular}{|c|c|c|c|c|c|c|c|c|c|c|}
\hline \multirow{3}{*}{\multicolumn{2}{|c|}{ UNIT OPERATION }} & \multicolumn{9}{|c|}{$\begin{array}{c}\text { ENTS - } 2 \\
\text { CWO and Vitrification }\end{array}$} \\
\hline & & \multicolumn{5}{|c|}{ Building Area } & \multicolumn{3}{|c|}{ Material \& Equipment Costs } & \multirow{2}{*}{$\begin{array}{c}\text { Total } \\
\text { Cost per } \\
\text { Unit Oprtn. } \\
\$ 1000 \\
\end{array}$} \\
\hline & & $\begin{array}{l}\text { Cost } \\
\text { Category } 1 \\
\text { sq.ft } \\
\end{array}$ & $\begin{array}{l}\text { Cost } \\
\text { Category } 2 \\
\quad \text { sq.ft } \\
\end{array}$ & $\begin{array}{l}\text { Cost } \\
\text { Category } 3 \\
\text { sq.ft }\end{array}$ & $\begin{array}{l}\text { Cost } \\
\text { Category } 4 \\
\quad \text { sq.ft } \\
\end{array}$ & $\begin{array}{c}\text { Total } \\
\text { Area Cost } \\
\$ 1000 \\
\end{array}$ & \begin{tabular}{|c|} 
Purchase \\
Cost \\
$\$ 1000$ \\
\end{tabular} & $\begin{array}{c}\text { Imstallation } \\
\text { Cost } \\
\$ 1000 \\
\end{array}$ & $\begin{array}{c}\text { Total } \\
\text { Cost } \\
\$ 1000\end{array}$ & \\
\hline & Administration Building Subsystem & 12,440 & 3,000 & & & 3,499 & 3,163 & 888 & 4,051 & 7,550 \\
\hline & Receiving \& Inspection Subsystem & & 24,227 & 2,970 & 14,839 & 38,966 & 18,494 & 6,775 & 25,268 & 64,234 \\
\hline & Aqucous Waste Treatment Subsystem & & 1,150 & 1,751 & 1,590 & 5,287 & 4,980 & 1,516 & 6,496 & 11,783 \\
\hline 4 & Organic Destruction Subsystem & & 1,128 & 1,717 & 1,560 & 5,187 & 5,750 & 1,476 & 7,227 & 12,414 \\
\hline \multicolumn{11}{|c|}{5 Residue \& Sludge Treatment Subsystem } \\
\hline 6 & Soft Debris Treatment Subsystem & & 1,606 & 2,444 & 2,220 & 7,381 & 8,590 & 1,454 & 10,044 & 17,425 \\
\hline \multicolumn{11}{|c|}{7 Complex Debris Treatment Subsystem } \\
\hline & Open Debris Treatment Subsystem & & 1,888 & 2,873 & 2,610 & 8,678 & 2,053 & 671 & 2,723 & 11,401 \\
\hline 9 & Bulk Soil Treatment Subsystem & & 1,606 & 2,444 & 2,220 & 7,381 & 2,822 & 832 & 3,655 & 11,036 \\
\hline 10 & Metal Decontamination Subsystem & & 641 & 169 & 1904 & 3,709 & 3,572 & 1,496 & 5,067 & 8,776 \\
\hline 11 & Special Waste & & 604 & 159 & 1792 & 3,491 & & & & 3,491 \\
\hline 12 & Mercury Amalgamation Subsystem & & 679 & 179 & 2,016 & 3,928 & 2,896 & 1,049 & 3,945 & 7,873 \\
\hline 13 & Lead Recovery Subsystem & & 830 & 219 & 2,464 & 4,800 & 4,027 & 1,155 & 5,182 & 9,982 \\
\hline 14 & Air Pollution Control Subsystem & & 955 & 1,453 & 1,320 & 4,389 & 2,401 & 885 & 3,285 & 7,674 \\
\hline 15 & Polymer Stabilization Subsystem & & 2,322 & 3,534 & 3,210 & 10,673 & 5,187 & 1,512 & 6,699 & 17,372 \\
\hline 16 & Vitrification & & 2,875 & 9,030 & 4,554 & 19,785 & 18,331 & 4,752 & 23,084 & 42,869 \\
\hline 17 & Certification \& Shipping Subsystem & & 28,277 & & & 11,876 & 4,829 & 2,837 & 7,666 & 19,542 \\
\hline 18 & Support Subsystem & 3360 & 16,883 & & & 7,696 & 4,796 & 1,057 & 5,852 & 13,548 \\
\hline \multirow[t]{3}{*}{19} & Metal Melting Subsystem & & 1,169 & 309 & 3,472 & 6,764 & 6,224 & 2,817 & 9,041 & 15,805 \\
\hline & Total Cost & & & & & 153,490 & & & 129,285 & 282,775 \\
\hline & & & Post Totals To T & ble 4-2 Item & & 3.4 .1 & & & 3.4 .2 & \\
\hline
\end{tabular}


Table B-2.4 : Annual Operating \& Maintenance Costs ENTS - 2 CWO and Vitrification

\begin{tabular}{|c|c|c|c|c|c|c|c|}
\hline \multirow{2}{*}{\multicolumn{2}{|c|}{ UNIT OPERATION }} & \multicolumn{6}{|c|}{$\begin{array}{c}\text { ENTS - 2 } \\
\text { CWO and Vitrification }\end{array}$} \\
\hline & & $\begin{array}{l}\text { Operating } \\
\text { FTE }\end{array}$ & $\begin{array}{l}\text { Utilities } \\
\$ 1000 \\
\end{array}$ & $\begin{array}{c}\text { Materials } \\
\$ 1000 \\
\end{array}$ & $\begin{array}{c}\text { Maintenance } \\
\text { Labor (1) } \\
\$ 1000 \\
\end{array}$ & $\begin{array}{c}\text { Maintenance } \\
\text { Materials (2) } \\
\$ \mathbf{\$ 1 0 0 0} \\
\end{array}$ & $\begin{array}{l}\text { Totals } \\
\$ 1000 \\
\end{array}$ \\
\hline 1 & Administration Building Subsystem & 32 & 5 & 164 & 318 & 127 & 445 \\
\hline 2 & Receiving \& Inspection Subsystem & 170 & 159 & 612 & 1,850 & 740 & 2,590 \\
\hline 3 & Aqueous Waste Treatment Subsystem & 5 & 300 & 1,705 & 873 & 349 & 1,222 \\
\hline 4 & Organic Destruction Subsystem & 12 & 298 & 297 & 1,438 & 575 & 2,013 \\
\hline 5 & Residue \& Sludge Treatment Subsystem & & & & & & \\
\hline 6 & Soft Debris Treatment Subsystem & 14 & 708 & 704 & 2,148 & 859 & 3,007 \\
\hline 7 & Complex Debris Treatment Subsystem & & & & & & \\
\hline 8 & Open Debris Treatment Subsystem & 8 & 25 & 100 & 360 & 144 & 504 \\
\hline 9 & Bulk Soil Treatment Subsystem & 2 & 22 & 14 & 140 & 56 & 196 \\
\hline 10 & Metal Decontamination Subsystem & 4 & 5 & 25 & 625 & 250 & 875 \\
\hline 11 & Special Waste & 3 & 12 & 200 & & & \\
\hline 12 & Mercury Amalgamation Subsystem & 4 & 2 & 10 & 508 & 203 & 711 \\
\hline 13 & Lead Recovery Subsystem & 8 & 73 & 50 & 705 & 282 & 987 \\
\hline 14 & Air Pollution Control Subsystem & 4 & 67 & 130 & 420 & 168 & 588 \\
\hline 15 & Polymer Stabilization Subsystem & 8 & 118 & 1,223 & 908 & 363 & 1,271 \\
\hline 16 & Vitrification & 18 & 65 & 1,177 & 1,833 & 733 & 2,566 \\
\hline 17 & Certification \& Shipping Subsystem & 37 & 12 & 70 & 483 & 193 & 676 \\
\hline 18 & Support Subsystem & 5 & & & 480 & 192 & 672 \\
\hline 19 & Metal Melting Subsystem & 5 & 79 & 33 & 623 & 249 & 872 \\
\hline & Unit cost (\$/unit) & $\$ 140,000$ & & & & & \\
\hline & Total Cost & 47,460 & 1950 & 6,514 & & & 19,195 \\
\hline & Post Totals To Table 4-2, Item & 5.1 & 5.2 & 5.3 & & & 5.4 \\
\hline
\end{tabular}

Notes:

1. Annual Maintenance Labor is $250 \%$ of maintenance material cost.

2. Maintenance Material is assumed to be $2 \%$ of equipment capital cost for the Bulk Soil Treatment subsystem, because the capital equipment is based on one shift operation.

3. Maintenance Material is assumed to be $10 \%$ of equipment capital cost for organic destruction and soft debris

4. Maintenance Material is assumed to be $4 \%$ of equipment capital cost for Administration, Receiving \& Inspection,

Grout Stabilization, Certification \& Shipping, and Support subsystems.

5. Maintenance Material is assumed to be $7 \%$ of equipment capital cost for all other subsystems 
Table B-2.5 : Decontamination \& Decommissioning Costs ENTS - 2 CWO and Vitrification

\begin{tabular}{|c|c|c|c|c|c|c|}
\hline & \multirow{3}{*}{ UNIT OPERATION } & \multicolumn{5}{|c|}{$\begin{array}{c}\text { ENTS - } 2 \\
\text { CWO and Vitrification }\end{array}$} \\
\hline & & \multicolumn{4}{|c|}{ Building Area } & \multirow[b]{2}{*}{$\begin{array}{c}\text { Total } \\
\text { Area Cost } \\
\$ 1000 \\
\end{array}$} \\
\hline & & \begin{tabular}{|c|} 
Cost \\
Category 1 \\
sq.ft
\end{tabular} & \begin{tabular}{|c|} 
Cost \\
Category 2 \\
sq.ft
\end{tabular} & \begin{tabular}{|c|} 
Cost \\
Category 3 \\
sq.ft
\end{tabular} & \begin{tabular}{|c|} 
Cost \\
Category 4 \\
sq.ft
\end{tabular} & \\
\hline 1 & Administration Building Subsystem & 12,440 & 3,000 & & & 6,948 \\
\hline 2 & Receiving \& Inspection Subsystem & & 24,227 & 2,970 & 14,839 & 18,916 \\
\hline 3 & Aqueous Waste Treatment Subsystem & & 1,150 & 1,751 & 1,590 & 2,021 \\
\hline 4 & Organic Destruction Subsystem & & 1,128 & 1,717 & 1,560 & 1,983 \\
\hline 5 & Residue \& Sludge Treatment Subsystem & & & & & \\
\hline 6 & Soft Debris Treatment Subsystem & & 1,606 & 2,444 & 2,220 & 2,822 \\
\hline 7 & Complex Debris Treatment Subsystem & & & & & \\
\hline 8 & Open Debris Treatment Subsystem & & 1,888 & 2,873 & 2,610 & 3,317 \\
\hline 9 & Bulk Soil Treatment Subsystem & & 1,606 & 2,444 & 2,220 & 2,822 \\
\hline 10 & Metal Decontamination Subsystem & & 641 & 169 & 1,904 & 1,222 \\
\hline 11 & Special Waste & & 604 & 159 & 1,792 & 1,150 \\
\hline 12 & Mercury Amalgamation Subsystem & & 679 & 179 & 2,016 & 1,293 \\
\hline 13 & Lead Recovery Subsystem & & 830 & 219 & 2,464 & 1,581 \\
\hline 14 & Air Pollution Control Subsystem & & 955 & 1,453 & 1,320 & 1,678 \\
\hline 15 & Polymer Stabilization Subsystem & & 2,322 & 3,534 & 3,210 & 4,080 \\
\hline 16 & Vitrification & & 2,875 & 9,030 & 4,554 & 7,407 \\
\hline 17 & Certification \& Shipping Subsystem & & 28,277 & & & 12,725 \\
\hline 18 & Support Subsystem & 3,360 & 16,883 & & & 9,109 \\
\hline \multirow[t]{3}{*}{19} & Metal Melting Subsystem & & 1,169 & 309 & 3,472 & 2,228 \\
\hline & Total Cost & & & & & 81,302 \\
\hline & & & Post Totals To T & able 4-2 Item & & 7.0 \\
\hline
\end{tabular}


Table H-2.6: Total Life-Cycle Cost (without Disposal) Estimate Summary for

Enhanced Integrated Non Thermal Treatment Systems (cost module ENTS-2)

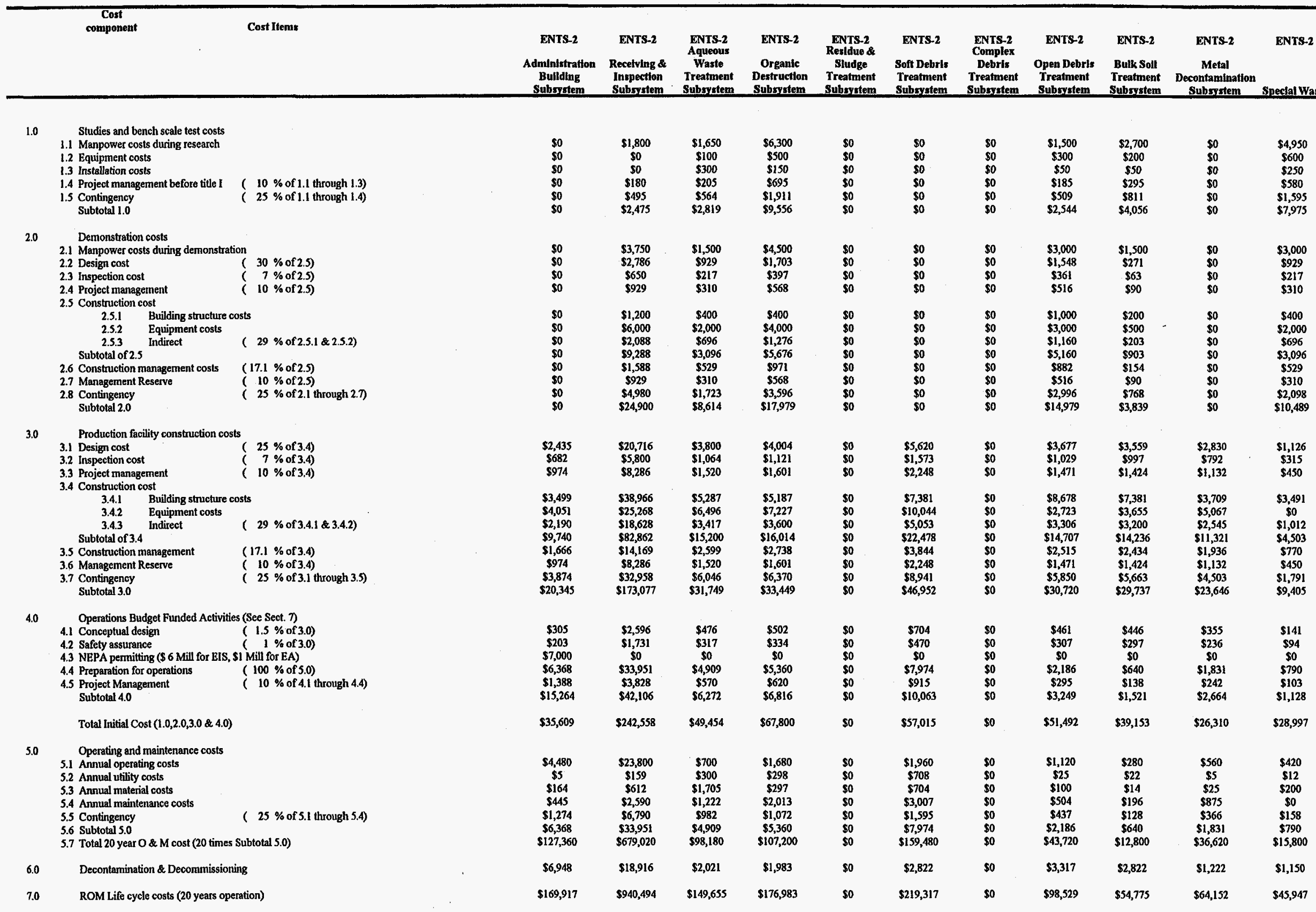


Table B-2.6 : Total Life-Cycle Cost (without Disposal) Estimate Summary for

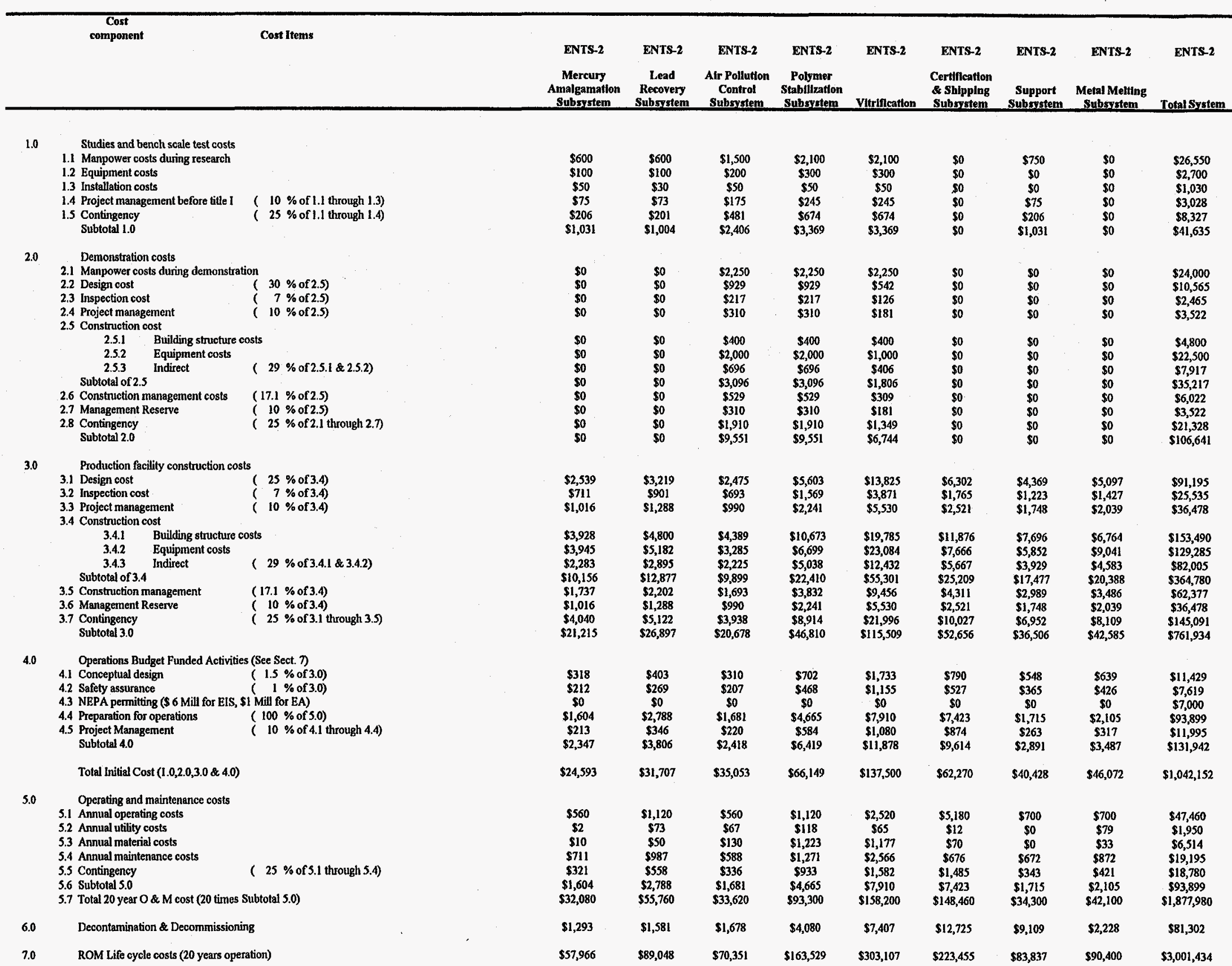




\section{B-3 ENTS-3 Cost Tables}

B-3.1. Equipment Purchase and Installation Budget Estimates

B-3.2. Development, Testing and Evaluation Cost Estimate

B-3.3. Building, Equipment and Installation Cost Summary

B-3.4. Annual Operating and Maintenance Costs

B-3.5. Decontamination and Decommissioning Costs

B-3.6 Total Life-Cycle Cost (without Disposal) Estimate for Enhanced Integrated Nonthermal Treatment Systems 
Table B-3.1: Equipment Purchase \& Installation Budget Estimates Enhanced Non-Thermal Treatment System ENTS - 3 CWO-and Polymer Stabilization

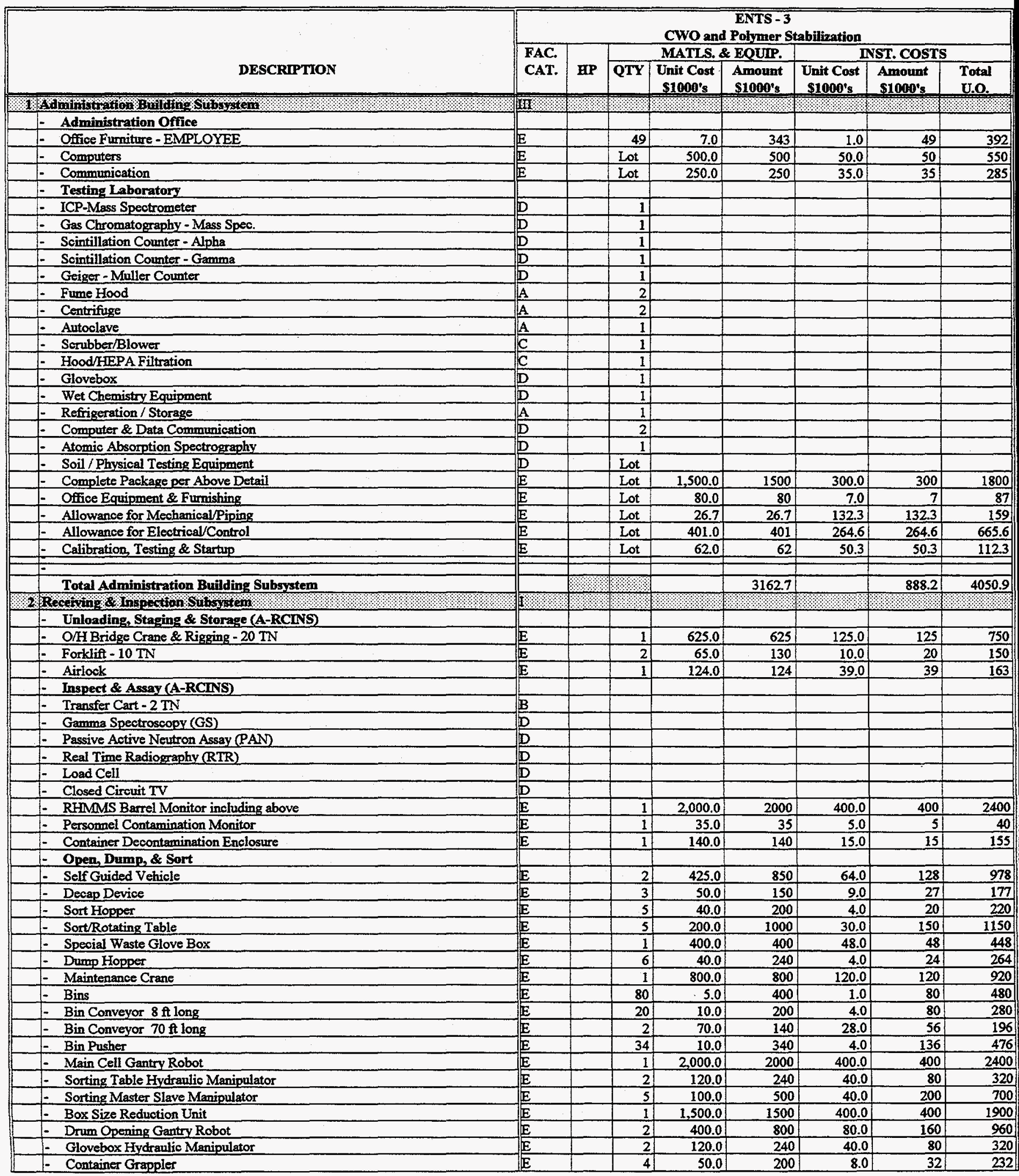


Table B-3.1: Equipment Purchase \& Installation Budget Estimates Enhanced Non-Thermal Treatment System ENTS - 3 CWO and Polymer Stabilization

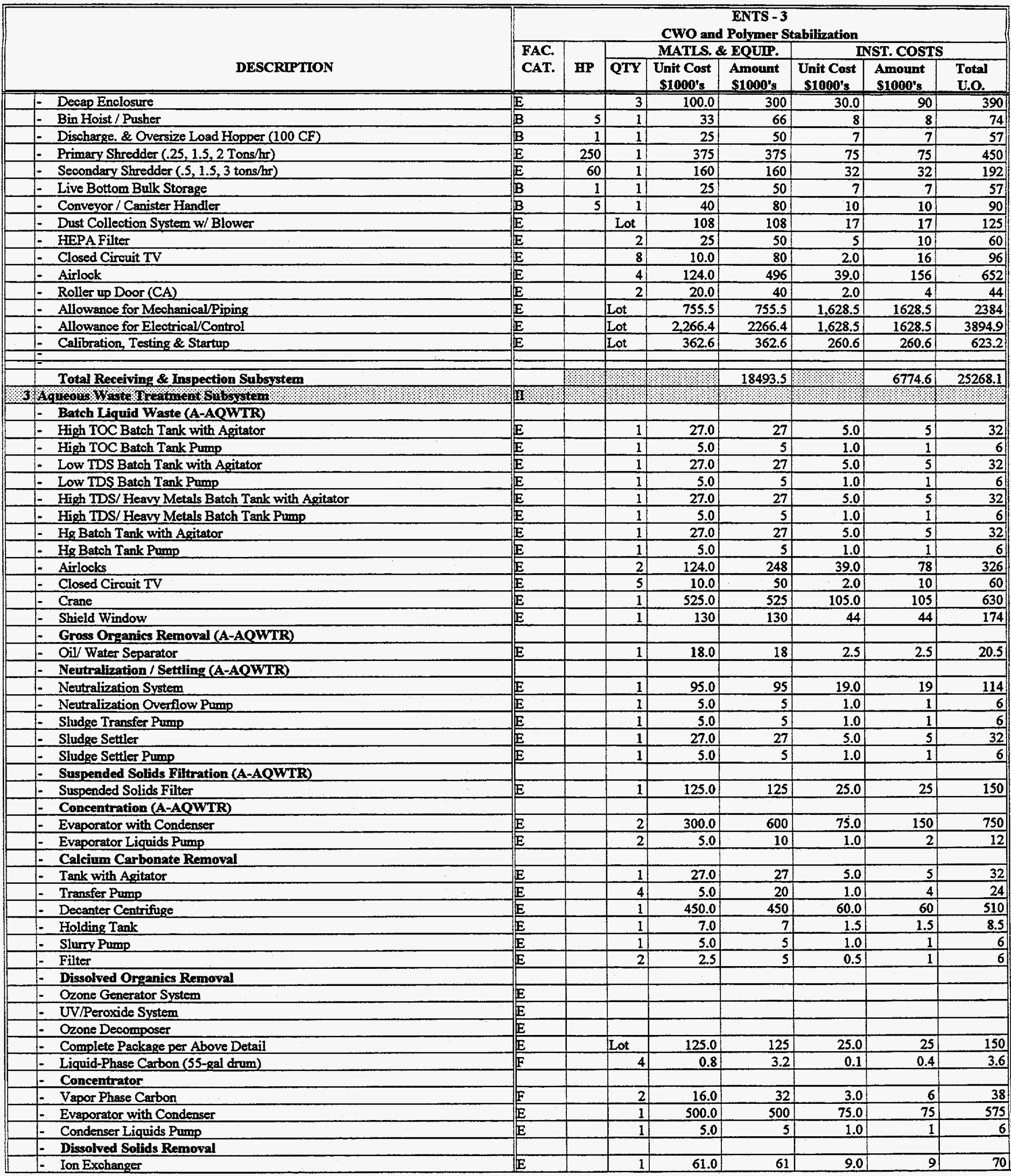


Table B-3.1: Equipment Purchase \& Installation Budget Estimates Enhanced Non-Thermal Treatment System ENTS - 3 CWO and Polymer Stabilization

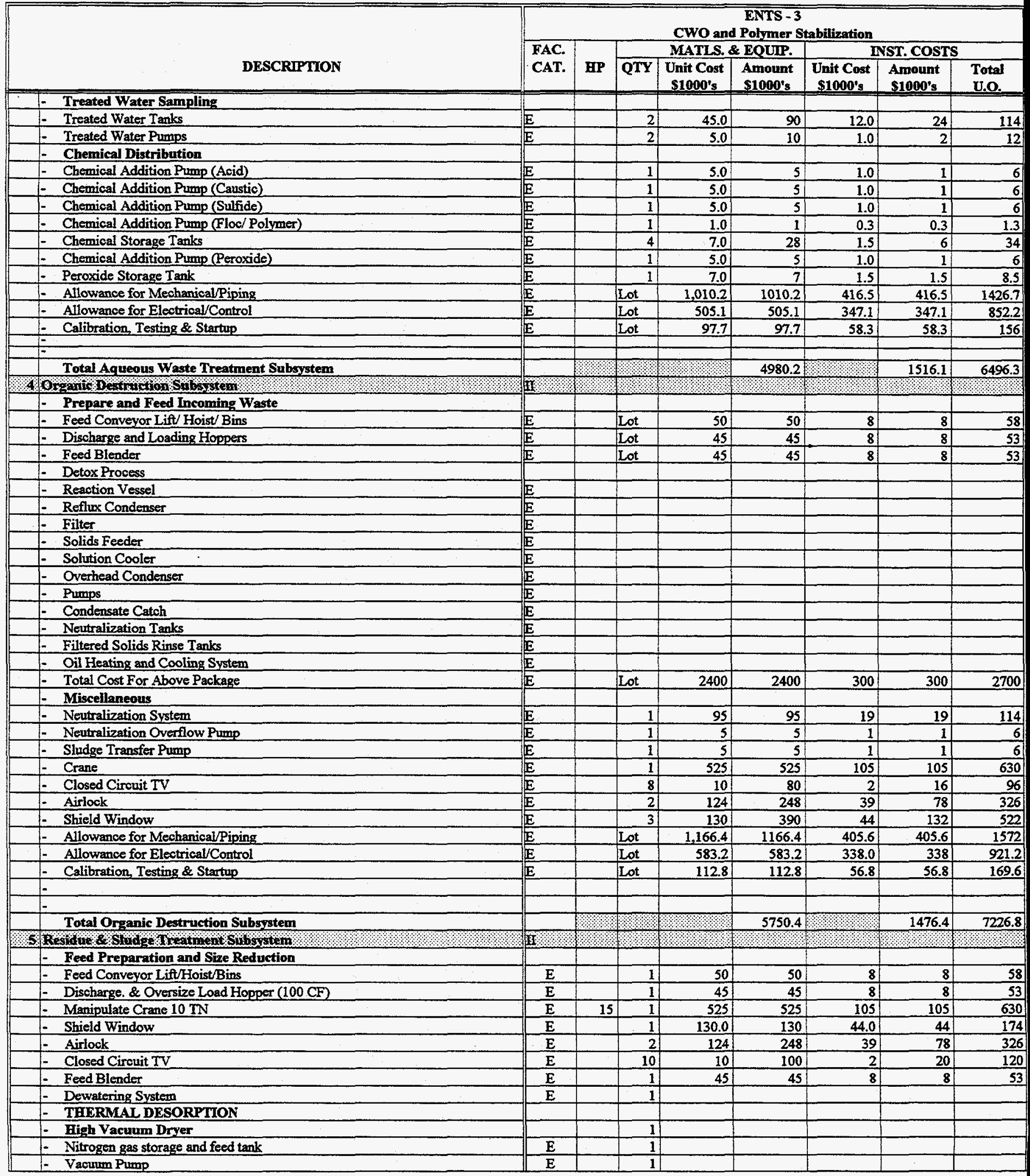


Table B-3.1: Equipment Purchase \& Installation Budget Estimates

Enhanced Non-Thermal Treatment System

ENTS - 3 CWO and Polymer Stabilization

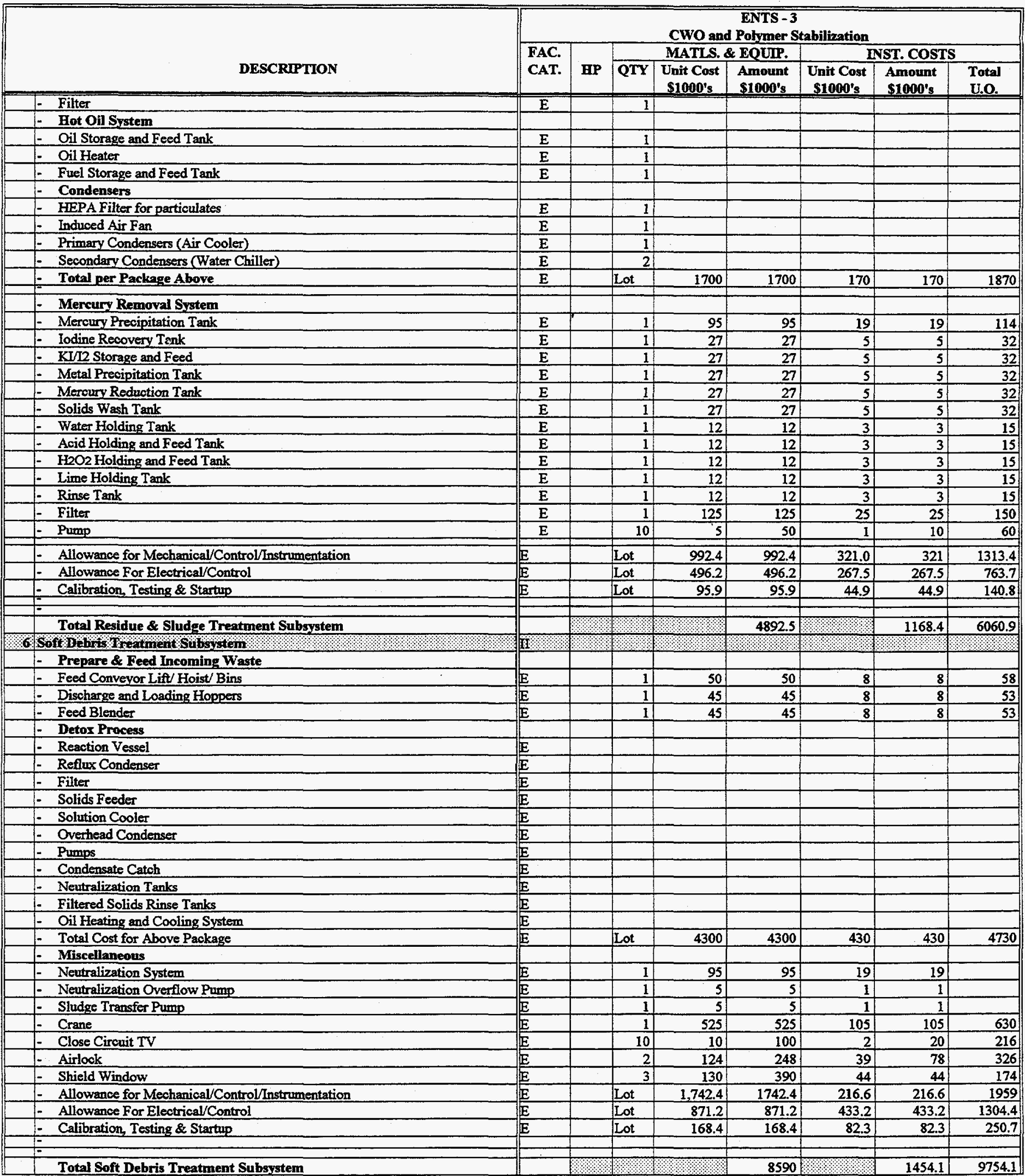


Table B-3.1: Equipment Purchase \& Installation Budget Estimates

Enhanced Non-Thermal Treatment System

ENTS - 3 CWO and Polymer Stabilization

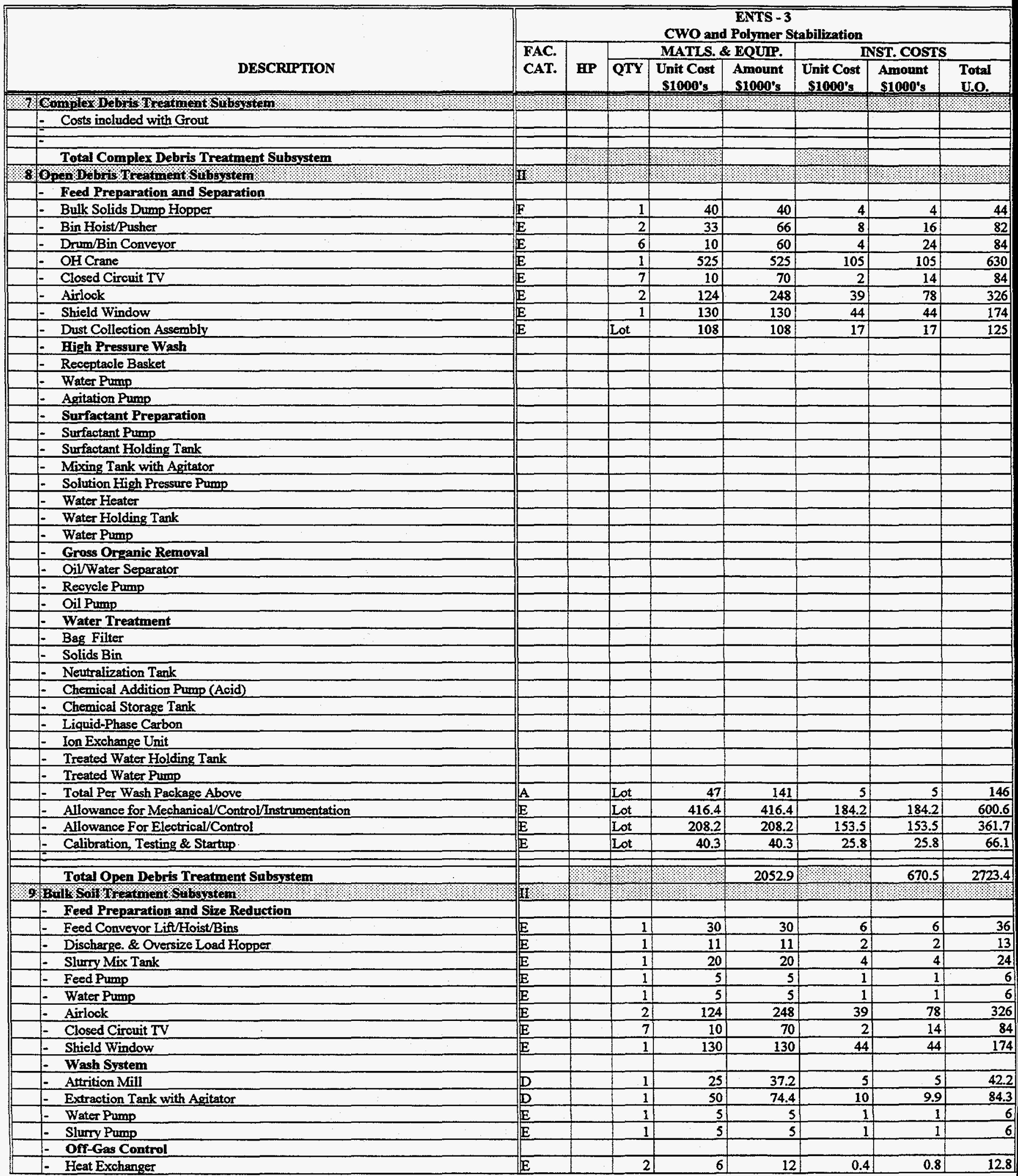


Table B-3.1: Equipment Purchase \& Installation Budget Estimates

Enhanced Non-Thermal Treatment System

ENTS - 3 CWO and Polymer Stabilization

\begin{tabular}{|c|c|c|c|c|c|c|c|c|c|}
\hline \multirow{3}{*}{\multicolumn{2}{|c|}{ DESCRIPTION }} & \multicolumn{8}{|c|}{$\begin{array}{c}\text { ENTS - 3 } \\
\text { CWO and Polymer Stabilization }\end{array}$} \\
\hline & & \multirow{2}{*}{$\begin{array}{l}\text { FAC. } \\
\text { CAT. }\end{array}$} & \multirow[b]{2}{*}{$\mathbf{H P}$} & \multicolumn{3}{|c|}{ MATLS. \& EQUIP. } & \multicolumn{3}{|c|}{ INST. CosTs } \\
\hline & & & & QTY & $\begin{array}{l}\text { Unit Cost } \\
\text { S1000's }\end{array}$ & $\begin{array}{l}\text { Amount } \\
\text { S1000's }\end{array}$ & $\begin{array}{l}\text { Unit Cost } \\
\text { S1000's }\end{array}$ & $\begin{array}{l}\text { Amount } \\
\text { S1000's }\end{array}$ & $\begin{array}{l}\text { Total } \\
\text { U.O. }\end{array}$ \\
\hline & - Solid/ Liquid Separation System & E & & & & & & & \\
\hline & - Vibrating Screen & E & & 1 & 6 & 6 & 1 & 1 & 7 \\
\hline & - Hydrocyclone & D & & 2 & 4.5 & 13.5 & 0.4 & 0.8 & 14.3 \\
\hline & - Countercurrent Solid/ Liquid Contactor & B & & 1 & 160 & 320 & 32 & 32 & 352 \\
\hline & - Water Pump & $E$ & & 1. & 5 & 5 & 1 & 1 & 6 \\
\hline & - Slurry Pump & $E$ & & 1 & 5 & 5 & 1 & 1 & 6 \\
\hline & - Diaphragm Pump & $E$ & & 1 & 1 & 1 & 0.2 & 0.2 & 1.2 \\
\hline & - Solids Bin & E & & 1 & 5 & 5 & 1 & 1 & 6 \\
\hline & - Overhead Crane & $E$ & & 1 & 525 & 525 & 105 & 105 & 630 \\
\hline & - Solubility Reduction Separator & & & & & & & & \\
\hline & - Phase Separator & B & & 1 & 4.8 & 9.6 & 1 & 1 & 10.6 \\
\hline & Additive Addition Pump & E & & 1] & 1 & 1 & 0.3 & 0.3 & 1.3 \\
\hline & - Surfactant Phase Pump & E & & 1 & 5 & 5 & 1. & 1 & 6 \\
\hline & - Water Phase Pump & $E$ & & 1 & 5 & 5 & 1 & 1 & 6 \\
\hline & - Storage Tanks (Water and Concentrate) & $\bar{E}$ & & 2 & 2 & 4 & 0.4 & 0.8 & 4.8 \\
\hline & - UitraFiltration Unit & E & & 1 & 50 & 50 & 10 & 10 & 60 \\
\hline & - Recycle Pump & E & & 1 & 5 & 5 & 1 & 1 & 6 \\
\hline & - Water Pump & E & & 1 & 5 & 5 & 1 & 1 & 6 \\
\hline & - Dissolved Air Floatation Unit & E & & 1 & 50 & 50 & 7.5 & 7.5 & 57.5 \\
\hline & - Surfactant Preparation & & & & & & & & \\
\hline & Water Hold Tank & E & & 1 & 2 & 2 & 0.2 & 0.2 & 2.2 \\
\hline & - Water Heater & $E$ & & 1 & 0.6 & 0.6 & 0.1 & 0.1 & 0.7 \\
\hline & - Surfactant Hold Tank & E & & 1. & 2 & 2 & 0.2 & 0.2 & 2.2 \\
\hline & - Water Pump & E & & 1 & 5 & 5 & 1 & 1 & 6 \\
\hline & - Surfactant Pump & E & & 1 & 5 & 5 & 1 & 1) & 6 \\
\hline & - Dilution Tank Agitator & E & & 1 & 1.5 & 1.5 & 0.2 & 0.2 & 1.7 \\
\hline & - Dilution Tank Pump & E & & 1 & 5 & 5 & 1 & 1 & 6 \\
\hline & - Surfactant Dilution Tank & $E$ & & 1 & 2.0 & 2 & 0.4 & 0.4 & 2.4 \\
\hline & - Drier & $E$ & & 1 & 100.0 & 100 & 30.0 & 30 & 130 \\
\hline & - Surfactant Separation & & & & & & & & \\
\hline & - Additive Pump & $E$ & & 4 & 1 & 4 & 0.3 & 1.2 & 5.2 \\
\hline & - Additive Tanks & E & & 1 & 3.2 & 3.2 & 0.5 & 0.5 & 3.7 \\
\hline & - Surfactant Separation Unit & $B$ & & 1 & 50 & 100 & 1 & 10 & 110 \\
\hline & - Recycle Pump & $E$ & & 1 & 5 & 5 & 1 & 1 & 6 \\
\hline & - Water Pump & E & & 1 & 5 & 5 & 1 & 1 & 6 \\
\hline & - Allowance for Mechanical/Control/nstrumentation & $E$ & & Lot & 572.4 & 572.4 & 228.7 & 228.7 & 801.1 \\
\hline & - Allowance For Electrical/Control & E & & Lot & 286.2 & 286.2 & 190.6 & 190.6 & 476.8 \\
\hline & - Calibration, Testing \& Startup & E & & Lot & 55.3 & 55.3 & 32.0 & 32 & 87.3 \\
\hline & 5 & & & & & & & & \\
\hline & Total Bulk Soil Treatment Subsystem & & & & & 2821.9 & & 832.4 & 3654.3 \\
\hline 10 & Mea Decontanifiation Subsysten & 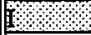 & & & 3 & & & 18 & 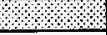 \\
\hline & - Pretreatment (GTCC-II) & & & & & & & & \\
\hline & - High Pressure Water Rinse Station & & & & & & & & \\
\hline & - Hot Air Dryer Station & & & & & & & & \\
\hline & - Chemical Storage Tank & & & & & & & & \\
\hline & - Chemical Mixing Tank & & & & & & & & \\
\hline & - Chemical Metering Pump & & & & & & & & \\
\hline & - Solution Pump & & & & & & & & \\
\hline & - Complete Package per Above Detail & $E$ & & Lot & 150.0 & 150 & 50.0 & 50 & 200 \\
\hline & - Shield Window & E & & 1 & 130 & 130 & 44 & 44 & 174 \\
\hline & - Closed Circuit TV & $\underline{E}$ & & 5 & 10.0 & 50 & 2.0 & 10 & 60 \\
\hline & - Size Reduction (GTCC-II) & & & & & & & & \\
\hline & - Hydraulic Manipulator & E & & 1 & 120.0 & 120 & 50.0 & 50 & 170 \\
\hline & - Gantry Robot & E & & 1 & 800.0 & 800 & 160.0 & 160 & 960 \\
\hline & - Size Reduction Table and Tools & E & & 1 & 205.0 & 205 & 40.0 & 40 & 245 \\
\hline & - Fugitive Dust Collection System & E & & 2 & 100.0 & 200 & 15.0 & 30 & 230 \\
\hline & - Master Slave Manipulator & E & & 1 & 65.0 & 65 & 15.0 & 15 & 80 \\
\hline & Airlock & $E$ & & 2 & 124.0 & 248 & 39.0 & 78 & 326 \\
\hline & - Assay/Inspection (GTCC-II) & & & & & & & & \\
\hline & - Assay Unit - Mini & E & & 11 & 500.0 & 500 & 100.0 & 100 & 600 \\
\hline & - Roller Conveyor & E & & 10 & 6.0 & 60 & 1.0 & 10 & 70 \\
\hline & I- Shielding Window & $\underline{E}$ & & 3 & 130.0 & 390 & 44.0 & 132 & 522 \\
\hline
\end{tabular}


Table B-3.1: Equipment Purchase \& Installation Budget Estimates

Enhanced Non-Thermal Treatment System

ENTS - 3 CWO and Polymer Stabilization

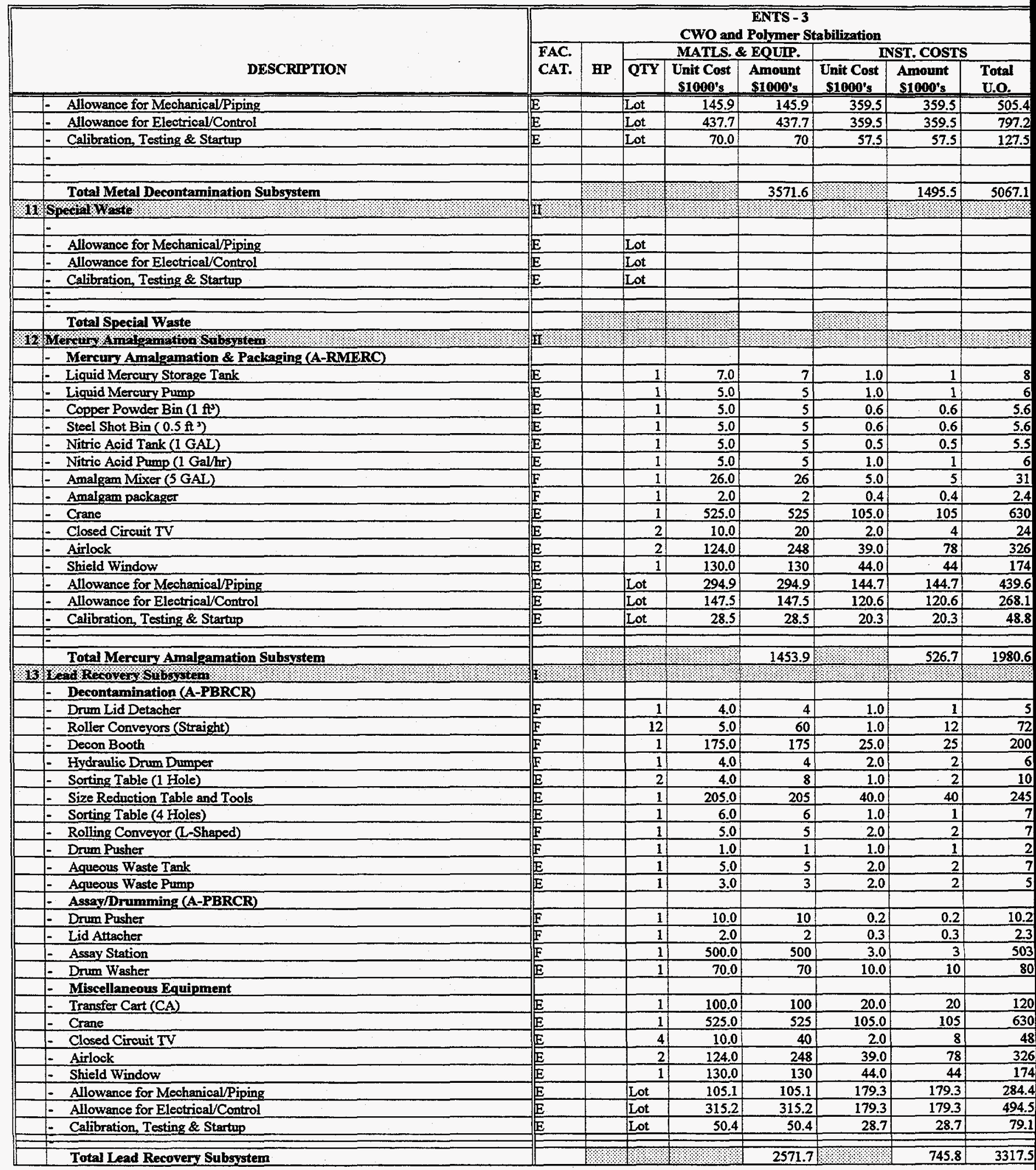


Table B-3.1: Equipment Purchase \& Installation Budget Estimates Enhanced Non-Thermal Treatment System ENTS - 3 CWO and Polymer Stabilization

\begin{tabular}{|c|c|c|c|c|c|c|c|c|}
\hline \multirow[b]{3}{*}{ DESCRIPTION } & \multicolumn{8}{|c|}{$\begin{array}{c}\text { ENTS - 3 } \\
\text { CWO and Polymer Stabilization }\end{array}$} \\
\hline & \multirow{2}{*}{$\begin{array}{l}\text { FAC. } \\
\text { CAT. }\end{array}$} & \multirow[b]{2}{*}{ HP } & \multicolumn{3}{|c|}{ MATLS. \& EQUIP. } & \multicolumn{3}{|c|}{ INST. COSTS } \\
\hline & & & QTY & \begin{tabular}{|c|} 
Unit Cost \\
S1000's \\
\end{tabular} & $\begin{array}{l}\text { Amount } \\
\text { S1000's }\end{array}$ & $\begin{array}{l}\text { Unit Cost } \\
\$ 1000 \text { 's }\end{array}$ & $\begin{array}{l}\text { Amount } \\
\text { S1000's }\end{array}$ & $\begin{array}{l}\text { Total } \\
\text { U.O. }\end{array}$ \\
\hline 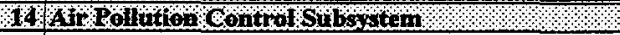 & II & & & & & & & \\
\hline - Particulate Filter & & & & & & & & \\
\hline - Baghouse Assembly & E & & 1 & 240.0 & 240 & 48.0 & 48. & 288 \\
\hline - Prefilter & $\mathbf{E}$ & & 2 & 26.0 & 52 & 12.0 & 24 & 76 \\
\hline - Inter Connection Ducts. & $\mathbf{E}$ & & Lot & 2.0 & 2 & 10.0 & 10 & 12 \\
\hline - Corona Reactor & & & & & & & & \\
\hline - Reactor & & & & & & & & \\
\hline - Skid \& Instrumentation & & & & & & & & \\
\hline - Power Supply & & & & & & & & \\
\hline - Cost per Package Above & $E$ & & Lot & 73.0 & 73 & 7.0 & 7 & 80 \\
\hline - Acid Gas Scrubber & & & & & & & & \\
\hline - Packing Tower & E & & 1 & 45.0 & 45 & 13.0 & 13 & 58 \\
\hline- Induced Fan & E & & 2 & 4.5 & 9 & 0.5 & 1 & 10 \\
\hline - Recycle Pump & $E$ & & 2 & 1.8 & 3.6 & 0.2 & 0.4 & 4 \\
\hline - Sludge Discharge Pump & E & & 2 & 2.7 & 5.4 & 0.5 & 1 & 6.4 \\
\hline - Caustic Feed Pump & $\mathrm{E}$ & & 2 & 0.9 & 1.8 & 0.2 & 0.4 & 2.2 \\
\hline - NaOH Transfer Pump & $E$ & & 2 & 0.9 & 1.8 & 0.2 & 0.4 & 2.2 \\
\hline - Caustic Storage Tank & $E$ & & 1 & 0.9 & 0.9 & 0.2 & 0.2 & 1.1 \\
\hline - Slurry Pump & $E$ & & 2 & 2.7 & 5.4 & 0.5 & 1 & 6.4 \\
\hline - Condensate Tank & $E$ & & 1 & 0.9 & 0.9 & 0.2 & 0.2 & 1.1 \\
\hline - NaOH Day Tank & $E$ & & 1 & 0.9 & 0.9 & 0.2 & 0.2 & 1.1 \\
\hline - Scrubber Solution Tank & $E$ & & 1 & 0.9 & 0.9 & 0.2 & 0.2 & 1.1 \\
\hline - Evaporator System & $E$ & & 1 & 54.0 & 54 & 16.2 & 16.2 & 70.2 \\
\hline - Hydrosonic Scrubber & $E$ & & 1 & 27.0 & 27 & 5.4 & 5.4 & 32.4 \\
\hline - Liquid/ Solid Separator & $E$ & & 1 & 18.0 & 18 & 3.6 & 3.6 & 21.6 \\
\hline - Mist Eliminator & $E$ & & 1 & 1.6 & 1.6 & 0.4 & 0.4 & 2 \\
\hline - Heat Exchanger & E & & 2 & 5.4 & 10.8 & 1.8 & 3.6 & 14.4 \\
\hline - Carbon Adsorption & & & & & & & & \\
\hline - Carbon Filter (GAC) & $\mathbf{F}$ & & 2 & 9.0 & 18 & 1.8 & 3.6 & 21.6 \\
\hline - Carbon Filter (Sublimated GAC) & $\mathrm{F}$ & & 2 & 9.0 & 18 & 1.8 & 3.6 & 21.6 \\
\hline Support System & & & & & & & & \\
\hline - Stack & $E$ & & 1 & 7.2 & 7.2 & 1.4 & 1.4 & 8.6 \\
\hline - Stack Monitors - Radioactive & E & & Lot & 56.0 & 56 & 14.4 & 14.4 & 70.4 \\
\hline - Stack Monitors - Non Radioactive & $E$ & & Lot & 27.0 & 27 & 10.8 & 10.8 & 37.8 \\
\hline - OH Crane & $\mathbf{E}$ & & 1 & 525.0 & 525 & 105.0 & 105 & 630 \\
\hline - Airlock & $E$ & & 2 & 124.0 & 248 & 39.0 & 78 & 326 \\
\hline - Closed Circuit TV & E & & 4 & 10.0 & 40 & 2.0 & 8 & 48 \\
\hline - Shield Window & $\mathbf{E}$ & & 1 & 130 & 130 & 44 & 44 & 174 \\
\hline - Allowance for Mechanical/Piping & E & & Lot & 487.0 & 487 & 243.0 & 243 & 730 \\
\hline - Allowance for Electrical/Control & $E$ & & Lot & 243.5 & 243.5 & 202.5 & 202.5 & 446 \\
\hline - Calibration, Testing \& Startup & $E$ & & Lot & 47.1 & 47.1 & 34.0 & 34 & 81.1 \\
\hline Total Air Pollution Control Subsystem & & & & & 2400.8 & & 884.5 & 3285.3 \\
\hline IS Rolymerstabilization Subsyetem & 1 & & & & 6 & & & 13 \\
\hline - Prepare \& Feed Incoming Waste (A-PLYMR) & & & & & & & & \\
\hline - Bin Hoist / Pusher & F & 5 & 1) & 100.0 & 100 & 8.0 & 8 & 108 \\
\hline - Discharge. \& Oversize Load Hopper (100 CF) & F & 1 & 1 & 90.0 & 90 & 9.0 & 9 & 99 \\
\hline - Tertiary Shredder & B & so & 1 & 75.0 & 150 & 10.0 & 10 & 160 \\
\hline - Live Bottom Bulk Storage & $\mathbf{F}$ & 1 & 1. & 90.0 & 90 & 9.0 & 9 & 99 \\
\hline - Conveyor / Canister Handler & F & 5 & 1 & 120.0 & 120 & 10.0 & 10 & 130 \\
\hline - Manipulation Crane - 5 TON & E & 10 & 1 & 525.0 & 525 & 105.0 & 105 & 630 \\
\hline - Dust Collection System w/ Blower & F & & 1 & 162.0 & 162 & 17.0 & 17 & 179 \\
\hline HEPA Filter & F & & 8 & 37.5 & 300 & 5.0 & 40 & 340 \\
\hline - Closed Circuit TV & E & & 13. & 10.0 & 130 & 2.0 & 26 & 156 \\
\hline Airlock & $E$ & & 2 & 124.0 & 248 & 39.0 & 78 & 326 \\
\hline - Shield Window & E & & 2 & 130 & 260 & 44 & 88 & 348 \\
\hline - Drying (A-PLYMR) & & & & & & & & \\
\hline - Drier & F & & 1 & 877.5 & 877.5 & 88.0 & 88 & 965.5 \\
\hline - Conveyor & F & 5 & 5 & 6.0 & 30 & 1.0 & 5 & $\mathbf{3 5}$ \\
\hline - Proportioning and Blending (A-PLYMR) & & & & & & & & \\
\hline - Polymer Pellets Storage Silo & E & & 4 & 37.5 & 150 & 7.0 & 28 & 178 \\
\hline - Weigh Station & E & & 2 & 30.0 & 60 & 2.0 & 4 & 64 \\
\hline - Blender & 正 & 2 & 1 & 79.5 & 79.5 & 5.0 & 5 & 84.5 \\
\hline
\end{tabular}


Table B-3.1: Equipment Purchase \& Installation Budget Estimates

Enhanced Non-Thermal Treatment System

ENTS - 3 CWO and Polymer Stabilization

\begin{tabular}{|c|c|c|c|c|c|c|c|c|c|}
\hline \multirow{3}{*}{\multicolumn{2}{|c|}{ DESCRIPTION }} & \multicolumn{8}{|c|}{$\begin{array}{l}\text { ENTS - } 3 \\
\text { CWO and Polymer Stabilization } \\
\end{array}$} \\
\hline & & \multirow{2}{*}{$\begin{array}{l}\text { FAC. } \\
\text { CAT. }\end{array}$} & \multirow[b]{2}{*}{$\mathbf{E P P}$} & \multicolumn{3}{|c|}{ MATLS. \& EQUIP. } & \multicolumn{3}{|c|}{ INST. COSTS } \\
\hline & & & & QTY & \begin{tabular}{|c|} 
Unit Cost \\
$\$ 1000 ' s$
\end{tabular} & $\begin{array}{l}\text { Amount } \\
\text { S1000's }\end{array}$ & $\begin{array}{c}\text { Unit Cost } \\
\text { S1000's }\end{array}$ & $\begin{array}{l}\text { Amount } \\
\text { S1000's }\end{array}$ & $\begin{array}{l}\text { Total } \\
\text { U.O. }\end{array}$ \\
\hline & Conveyor & F & 5 & 11 & 6.0 & 66 & 1.0 & 11 & $\overline{77}$ \\
\hline & Encapsulation (A-PLYMR) & & & & & & & & \\
\hline & - Extruder & F & 100 & 1 & 945.0 & 945 & 22.7 & 22.7 & 967.7 \\
\hline & - Activated Carbon & F & & 4 & 15.0 & 60 & 1.0 & 4 & 64 \\
\hline & - Drum Cooling (A-PLYMR) & & & & & & & & \\
\hline & - Induced Draft Fan/ Ducting & F & & 8 & 24.0 & 192 & 2.0 & 16 & 208 \\
\hline & - Enclosure & E & & 1 & 181.5 & 181.5 & 8.0 & 8 & 189.5 \\
\hline & - Drum Capping and Washing (A-PLYMR) & & & & & & & & \\
\hline & - Drum Wash Cap & E & & 4 & 105.0 & 420 & 15.0 & 60 & 480 \\
\hline & - Flush Water Collection and Conveying & E & & 2 & 45.0 & 90 & 18.0 & 36 & 126 \\
\hline & - Conveyor & F & 5 & 39 & 6.0 & 234 & 1.0 & 39 & 273 \\
\hline & - Allowance for Mechanical/Piping & $E$ & & Lot & 278.0 & 278 & 363.4 & 363.4 & 641.4 \\
\hline & - Allowance for Electrical/Control & $E$ & & Lot & 834.1 & 834.1 & 363.4 & 363.4 & 1197.5 \\
\hline & $\equiv$ Calibration, Testing \& Startup & E & & Lot & 133.5 & 133.5 & 58.1 & 58.1 & 191.6 \\
\hline & Total Polymer Stabilization Subsystem & & & & & 6806.1 & & 1511.6 & 8317.7 \\
\hline 16 & Centification \& Shipping Sulthsystesn & 1 & & \% & ma & 60 & ڤ & 1 & 1 \\
\hline & - Incoming Material Storage (B-CSEIIP) & & & & & & & & \\
\hline & - Overhead Bridge Crane $20 \mathrm{TN}$ & $E$ & & 1 & 625.0 & 625 & 125.0 & 125 & 750 \\
\hline & Forklift $10 \mathrm{TN}$ & E & & 1 & 65.0 & 65 & 10.0 & 10 & 75 \\
\hline & - Inspect \& Assay (B-CSBIP) & & & & & & & & \\
\hline & - Transfer Cart 2 TN & $E$ & & & & & & & \\
\hline & - Real Time Radiography (ND) & & & & & & & & \\
\hline & - Passive Active Neutron (ND) & & & & & & & & \\
\hline & - Segmented Gamma Spectroscopy (ND) & & & & & & & & \\
\hline & - Complete Package per Above & $E$ & & 2 & $1,500.0$ & 3000 & 150.0 & 300 & 3300 \\
\hline & - Self Guided Vehicle (ND) & $E$ & & 1 & 425.0 & 425 & 850.0 & 850 & 1275 \\
\hline & - Truck Loading (B-CSHIP) & & & & & & & & \\
\hline & - Forklift $10 \mathrm{TN}$ & E & & 10 & 65.0 & 650 & 10.0 & 100 & 750 \\
\hline & - Roll-up Door & $E$ & & 2 & 20.0 & 40 & 2.0 & 4 & 44 \\
\hline & - Bridge Crane & E & & 1 & 525.0 & 525 & 105.0 & 105 & 630 \\
\hline & - Roller Conveyor (CA) & E & & 20 & 2.0 & 40 & 1.0 & 20 & 60 \\
\hline & - Allowance for Mechanical/Piping & $E$ & & Lot & 268.5 & 268.5 & 757.0 & 757 & 1025.5 \\
\hline & - Allowance for Electrical/Control & $\underline{E}$ & & Lot & 805.5 & 805.5 & 757.0 & 757 & 1562.5 \\
\hline & $=$ Calibration, Testing \& Startup & E & & Lot & 128.9 & 128.9 & 121.1 & 121.1 & 250 \\
\hline & Total Certification \& Shipping Subsystem & & & 1 & & 6572.9 & & 3149.1 & 9722 \\
\hline
\end{tabular}


Table B-3.1: Equipment Purchase \& Installation Budget Estimates

Enhanced Non-Thermal Treatment System

ENTS - 3 CWO and Polymer Stabilization

\begin{tabular}{|c|c|c|c|c|c|c|c|c|c|}
\hline & \multirow[b]{3}{*}{ DESCRIPTION } & \multicolumn{8}{|c|}{$\begin{array}{c}\text { ENTS - 3 } \\
\text { CWO and Polymer Stabilization }\end{array}$} \\
\hline & & \multirow{2}{*}{$\begin{array}{l}\text { FAC. } \\
\text { CAT. }\end{array}$} & \multirow[b]{2}{*}{$\mathbf{H P}$} & \multicolumn{3}{|c|}{ MATLS. \& EQUTP. } & \multicolumn{3}{|c|}{ INST. COSTS } \\
\hline & & & & QTY & $\begin{array}{c}\text { Unit Cost } \\
\text { S1000's }\end{array}$ & $\begin{array}{l}\text { Amount } \\
\text { S1000's }\end{array}$ & $\begin{array}{c}\text { Unit Cost } \\
51000^{\prime} s\end{array}$ & $\begin{array}{l}\text { Amount } \\
\$ 1000 \text { 's }\end{array}$ & $\begin{array}{l}\text { Total } \\
\text { U.O. }\end{array}$ \\
\hline 21 & 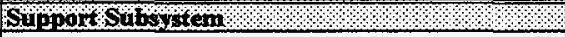 & II. & & & ग1, & & 7 & & \\
\hline & - Electrical Distribution and MCC & & & & & & & & \\
\hline & - Control Panel - FT & $\underline{E}$ & & 110 & 10.0 & 1100 & 2.0 & 220 & 1320 \\
\hline & - Emergency Power Generator & $E$ & & 1 & 500.0 & 500 & 100.0 & 100 & 600 \\
\hline & - Main Control Panel & $\mathbf{E}$ & & 20 & 10.0 & 200 & 2.0 & 40 & 240 \\
\hline & - Motor Control Center & $\mathbf{E}$ & & 100 & 1.5 & 150 & 0.5 & 50 & 200 \\
\hline & - Stand by Emergency Power & $E$ & & 1 & 230.0 & 230 & 80.0 & 80 & 310 \\
\hline & - Utilities and Mechanical & & & & & & & & \\
\hline & - Breathing Air Package & E. & & Lot & 70.0 & 70 & 30.0 & 30 & 100 \\
\hline & - Compressed Air Package & $\mathrm{E}$ & & Lot & 100.0 & 100 & 25.0 & 25 & 125 \\
\hline & - Instrument Air & $\mathbb{E}$ & & 1 & 70.0 & 70 & 30.0 & 30 & 100 \\
\hline & - Instrument Air Package & E & & Lot & 90.0 & 90 & 25.0 & 25 & 115 \\
\hline & - Plant Steam/Boiler & $E$ & & 1 & 75.0 & 75 & 25.0 & 25 & 100 \\
\hline & - Service Water & $\underline{E}$ & & 1 & 130.0 & 130 & 40.0 & 40 & 170 \\
\hline & - Sump Pump & $\underline{E}$ & 3 & 10 & 5.0 & 50 & 2.0 & 20 & 70 \\
\hline & - Cooling Tower & $E$ & & 1 & 200.0 & 200 & 10.0 & 10 & 210 \\
\hline & - Cooling Water Pumps & $\mathbf{E}$ & 3 & 2 & 15.0 & 30 & 3.0 & 6 & 36 \\
\hline & - Heating, Ventilation \& Exhaust & & & & & & & & \\
\hline & - Included with Building Cost & & & & & & & & \\
\hline & - Radiation Monitoring & & & & & & & & \\
\hline & - Air Monitors & $\mathbf{E}$ & & 44 & 10.0 & 440 & 2.0 & 88 & 528 \\
\hline & - Area Monitoring & $\mathbf{E}$ & & 44 & 4.5 & 198 & 1.0 & 44 & 242 \\
\hline & - Personnel Monitoring Portals & $E$ & & 6 & 35.0 & 210 & 5.0 & 30 & 240 \\
\hline & - Portal Monitors & $\underline{E}$ & & 6 & 35.0 & 210 & 5.0 & 30 & 240 \\
\hline & - Allowance for Mechanical/Piping & $E$ & & Lot & 40.5 & 40.5 & 8.9 & 8.9 & 49.4 \\
\hline & - Allowance for Electrical/Control & $\mathrm{E}$ & & Lot & 608.0 & 608 & 134.0 & 134 & 742 \\
\hline & - Calibration, Testing \& Startup & $\underline{E}$ & & Lot & 94.0 & 94 & 20.7 & 20.7 & 114.7 \\
\hline & F & & & & & & & & \\
\hline & Total Support Subsystem & & & +? & & 4795.5 & & 1056.6 & 5852.1 \\
\hline 3 & २७२? & 8 & & & 3 & +1. & & & \\
\hline
\end{tabular}


Table B-3.2 : Development, Testing \& Evaluation Cost Estimates ENTS - 3 CWO and Polymer Stabilization

\begin{tabular}{|c|c|c|c|c|c|c|c|c|c|c|}
\hline \multirow{2}{*}{\multicolumn{2}{|c|}{ UNIT OPERATION }} & \multicolumn{9}{|c|}{$\begin{array}{c}\text { ENTS - 3 } \\
\text { CWO and Polymer Stabilization }\end{array}$} \\
\hline & & $\begin{array}{c}\text { Primary } \\
\text { Paper } \\
\text { Evaluation } \\
\text { Research } \\
\text { Manpower } \\
\end{array}$ & \begin{tabular}{|c|} 
Bench \\
Scale \\
Studies \\
Research \\
Manpower \\
\end{tabular} & $\begin{array}{c}\text { Sec. } \\
\text { Paper } \\
\text { Evaluation } \\
\text { Research } \\
\text { Manpower } \\
\end{array}$ & $\begin{array}{c}\text { Total } \\
\text { Research } \\
\text { Manpower }\end{array}$ & $\begin{array}{c}\text { Bench } \\
\text { Scale } \\
\text { Studies } \\
\text { Equipment } \\
\$ 1000 \\
\end{array}$ & $\begin{array}{c}\text { Bench } \\
\text { Scale } \\
\text { Studies } \\
\text { Installation } \\
\$ 1000 \\
\end{array}$ & \begin{tabular}{|c|} 
Pilot \\
Plant \\
Mock-up \\
Test Demo. \\
Manpower \\
\end{tabular} & $\begin{array}{c}\text { Pilot } \\
\text { Plant } \\
\text { Mock-up } \\
\text { Test Bldg. } \\
\text { \$1000 } \\
\end{array}$ & \begin{tabular}{|c|} 
Pilot \\
Plant \\
Mock-up \\
Test Equip. \\
\$1000 \\
\end{tabular} \\
\hline \multicolumn{2}{|r|}{\begin{tabular}{l|l}
1 & Administration Building Subsystem \\
\end{tabular}} & & & & & 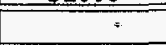 & & & & \\
\hline$\frac{1}{2}$ & Receiving \& Inspection Subsystem & 12 & & & 12 & & & 25 & 1200 & 6000 \\
\hline 3 & Aqueous Waste Treatment Subsystem & 5 & 3 & 3 & 11 & 100 & 300 & 10 & 400 & 2000 \\
\hline 4 & Organic Destruction Subsystem & 18 & 20 & 4 & 42 & 500 & 150 & 30 & 400 & 4000 \\
\hline 5 & Residue \& Sludge Treatment Subsystem & 13 & 8 & 4 & 25 & 500 & 150 & 30 & 500 & 2000 \\
\hline & \multicolumn{10}{|l|}{6 Soft Debris Treatment Subsystem } \\
\hline \multicolumn{11}{|c|}{7 Complex Debris Treatment Subsystem } \\
\hline 8 & Open Debris Treatment Subsystem & 2 & 6 & 2 & 10 & 300 & 50 & 20 & 1000 & 3000 \\
\hline$\frac{6}{9}$ & Bulk Soil Treatment Subsystem & 10 & 6 & 2 & 18 & 200 & 50 & 10 & 200 & 500 \\
\hline 10 & Metal Decontamination Subsystem & & & & & & & & & \\
\hline 11 & Special Waste & 8 & 20 & 5 & 33 & 600 & 250 & 20 & 400 & 2000 \\
\hline 12 & Mercury Amalgamation Subsystem & 1 & 3 & & 4 & 100 & 50 & & & \\
\hline 13 & Lead Recovery Subsystem & 2 & 2 & & 4 & 100 & 30 & & & \\
\hline 14 & Air Pollution Control Subsystem & 5 & 3 & 2 & 10 & 200 & 50 & 15 & 400 & 2000 \\
\hline 15 & Polymer Stabilization Subsystem & 2 & 8 & 4 & 14 & 300 & 50 & 15 & 400 & 2000 \\
\hline \multirow{5}{*}{$\frac{16}{17}$} & Certification \& Shipping Subsystem & & & & & & & & & \\
\hline & Support Subsystem & 5 & & & 5 & & & & & \\
\hline & Unit Cost (\$/Unit) & & & & $\$ 150,000$ & (x) & & $\$ 150,000$ & & \\
\hline & Total Cost & & & & 28,200 & 2,900 & 1,130 & 26,250 & 4,900 & 23,500 \\
\hline & & Post Totals To I & Table 4-3. Item & & 1.1 & 1.2 & 1.3 & 2.1 & 2.5 .1 & 2.5 .2 \\
\hline
\end{tabular}


Table B-3.3 : Building, Equipment \& Installation Cost Summary ENTS - 3 CWO and Polymer Stabilization

\begin{tabular}{|c|c|c|c|c|c|c|c|c|c|c|}
\hline & \multirow{3}{*}{ UNIT OPERATION } & \multicolumn{9}{|c|}{$\begin{array}{l}\text { ENTS - 3 } \\
\text { CWO and Polymer Stabilization } \\
\end{array}$} \\
\hline & & \multicolumn{5}{|c|}{ Building Area } & \multicolumn{3}{|c|}{ Material \& Equipment Costs } & \multirow{2}{*}{$\begin{array}{c}\text { Total } \\
\text { Cost per } \\
\text { Unit Oprtn. } \\
\$ 1000 \\
\end{array}$} \\
\hline & & $\begin{array}{c}\text { Cost } \\
\text { Category } 1 \\
\text { sq.ft } \\
\end{array}$ & $\begin{array}{c}\text { Cost } \\
\text { Category } 2 \\
\text { sq.ft } \\
\end{array}$ & $\begin{array}{c}\text { Cost } \\
\text { Category } 3 \\
\text { sg.ft }\end{array}$ & $\begin{array}{c}\text { Cost } \\
\text { Category } 4 \\
\text { sq.ft } \\
\end{array}$ & $\begin{array}{c}\text { Total } \\
\text { Area Cost } \\
\$ 1000 \\
\end{array}$ & 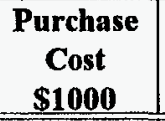 & $\begin{array}{c}\text { Installation } \\
\text { Cost } \\
\$ 1000 \\
\end{array}$ & $\begin{array}{c}\text { Total } \\
\text { Cost } \\
\$ 1000 \\
\end{array}$ & \\
\hline 1 & Administration Building Subsystem & 12,440 & 3,000 & & & 3499 & 3162.7 & 888.2 & 4051 & \begin{tabular}{|r|}
7550 \\
\end{tabular} \\
\hline 2 & Receiving \& Inspection Subsystem & & 24,227 & 2,970 & 14,839 & 38966 & 18493.5 & 6774.6 & 25268 & 64234 \\
\hline 3 & Aqueous Waste Treatment Subsystem & & 1,150 & 1,751 & 1,590 & 5287 & 4980.2 & 1516.1 & 6496 & 11783 \\
\hline 4 & Organic Destruction Subsystem & & 1,128 & 1,717 & 1,560 & 5187 & 5750.4 & 1476.4 & 7227 & 12414 \\
\hline 5 & Residue \& Sludge Treatment Subsystem & & 2,040 & 3,105 & 2,820 & 9376 & 4892.5 & 1168.4 & 6061 & 15437 \\
\hline 6 & Soft Debris Treatment Subsystem & & 1,606 & 2,444 & 2,220 & 7381 & 8590 & 1454.1 & 10044 & 17425 \\
\hline 7 & Complex Debris Treatment Subsystem & & & & & & & & & \\
\hline 8 & Open Debris Treatment Subsystem & & 1,888 & 2,873 & 2,610 & 8678 & 2052.9 & 670.5 & 2723 & 11401 \\
\hline 9 & Bulk Soil Treatment Subsystem & & 1,606 & 2,444 & 2,220 & 7381 & 2821.9 & 832.4 & 3654 & 11035 \\
\hline 10 & Metal Decontamination Subsystem & & 641 & 169 & 1904 & 3709 & 3571.6 & 1495.5 & 5067 & 8776 \\
\hline 11 & Special Waste & & 604 & 159 & 1792 & 3491 & & & & 3491 \\
\hline 12 & Mercury Amalgamation Subsystem & & 1020 & 1552 & 1410 & 4688 & 1453.9 & 526.7 & 1981 & 6669 \\
\hline 13 & Lead Recovery Subsystem & & & & & & 2571.7 & 745.8 & 3318 & 3318 \\
\hline 14 & Air Pollution Control Subsystem & & 955 & $.1,453$ & 1,320 & 4389 & 2400.8 & 884.5 & 3285 & 7674 \\
\hline 15 & Polymer Stabilization Subsystem & & 3,097 & 8,278 & 5,250 & 20159 & 6806.1 & 1511.6 & 8318 & 28477 \\
\hline 16 & Certification \& Shipping Subsystem & & 28,277 & & & 11876 & 6572.9 & 3149.1 & 9722 & 21598 \\
\hline \multirow[t]{3}{*}{17} & Support Subsystem & 3360 & 16,883 & & & 7696 & 4795.5 & 1056.6 & 5852 & 13548 \\
\hline & Total Cost & & & & & 141,763 & & & 103,067 & 244,830 \\
\hline & & \multicolumn{4}{|c|}{ Post Totals To Table 4-3, Item } & 3.4 .1 & & & 3.4 .2 & \\
\hline
\end{tabular}


Table B-3.4 : Annual Operating \& Maintenance Costs

\section{ENTS - 3 CWO and Polymer Stabilization}

\begin{tabular}{|c|c|c|c|c|c|c|c|}
\hline \multirow{2}{*}{\multicolumn{2}{|c|}{ UNIT OPERATION }} & \multicolumn{6}{|c|}{$\begin{array}{c}\text { ENTS - } 3 \\
\text { CWO and Polymer Stabilization } \\
\end{array}$} \\
\hline & & $\begin{array}{l}\text { Operating } \\
\text { FTE }\end{array}$ & $\begin{array}{l}\text { Utilities } \\
\$ 1000 \\
\end{array}$ & $\begin{array}{c}\text { Materials } \\
\$ 1000 \\
\end{array}$ & $\begin{array}{c}\text { Maintenance } \\
\text { Labor (1) } \\
\$ 1000 \\
\end{array}$ & $\begin{array}{c}\text { Maintenance } \\
\text { Materials (2) } \\
\$ 1000 \\
\end{array}$ & $\begin{array}{l}\text { Totals } \\
\$ 1000 \\
\end{array}$ \\
\hline 1 & Administration Building Subsystem & 32 & 5 & 164 & 318 & 127 & -445 \\
\hline 2 & Receiving \& Inspection Subsystem & 170 & 159 & 612 & 1,850 & 740 & 2,590 \\
\hline 3 & Aqueous Waste Treatment Subsystem & 5 & 300 & 1,705 & 873 & 349 & 1,222 \\
\hline 4 & Organic Destruction Subsystem & 12 & 298 & 297 & 1,438 & 575 & 2,013 \\
\hline 5 & Residue \& Sludge Treatment Subsystem & 8 & 21 & 1 & 855 & 342 & 1,197 \\
\hline 6 & Soft Debris Treatment Subsystem & 14 & 708 & 704 & 2,148 & 859 & 3,007 \\
\hline 7 & Complex Debris Treatment Subsystem & & & & & & \\
\hline 8 & Open Debris Treatment Subsystem & 8 & 25 & 100 & 360 & 144 & 504 \\
\hline 9 & Bulk Soil Treatment Subsystem & 2 & 22 & 14 & 140 & 56 & 196 \\
\hline 10 & Metal Decontamination Subsystem & 4 & 5 & 25 & 625 & 250 & 875 \\
\hline 11 & Special Waste & 3 & 12 & 200 & & & \\
\hline 12 & Mercury Amalgamation Subsystem & 1 & 2 & 10 & 255 & 102 & 357 \\
\hline 13 & Lead Recovery Subsystem & 3 & 73 & 50 & 450 & 180 & 630 \\
\hline 14 & Air Pollution Control Subsystem & 4 & 67 & 130 & 420 & 168 & 588 \\
\hline 15 & Polymer Stabilization Subsystem & 56 & 177 & 3,344 & 1,190 & 476 & 1,666 \\
\hline 16 & Certification \& Shipping Subsystem & 88 & 12 & 70 & 658 & 263 & 921 \\
\hline \multirow[t]{4}{*}{17} & Support Subsystem & 5 & & & 480 & 192 & 672 \\
\hline & Unit cost (\$/unit) & $\$ 140,000$ & & & & & \\
\hline & Total Cost & 58,135 & 1886 & 7,426 & & & 16,883 \\
\hline & Post Totals To Table 4-3, Item & 5.1 & 5.2 & 5.3 & & & 5.4 \\
\hline
\end{tabular}

Notes:

1. Annual Maintenance Labor is $250 \%$ of maintenance material cost.

2. Maintenance Material is assumed to be $2 \%$ of equipment capital cost for the Bulk Soil Treatment subsystem, because the capital equipment is based on one shift operation.

3. Maintenance Material is assumed to be $10 \%$ of equipment capital cost for organic destruction and soft debris.

4. Maintenance Material is assumed to be $4 \%$ of equipment capital cost for Administration, Receiving \& Inspection,

Grout Stabilization, Certification \& Shipping, and Support subsystems.

5. Maintenance Material is assumed to be $7 \%$ of equipment capital cost for all other subsystems 
Table B-3.5 : Decontamination \& Decommissioning Costs ENTS - 3 CWO and Polymer Stabilization

\begin{tabular}{|c|c|c|c|c|c|c|}
\hline & \multirow[b]{3}{*}{ UNIT OPERATION } & \multicolumn{5}{|c|}{$\begin{array}{c}\text { ENTS - 3 } \\
\text { CWO and Polymer Stabilization }\end{array}$} \\
\hline & & \multicolumn{4}{|c|}{$\begin{array}{l}\text { Building Area } \\
\end{array}$} & \multirow[b]{2}{*}{$\begin{array}{c}\text { Total } \\
\text { Area Cost } \\
\$ 1000 \\
\end{array}$} \\
\hline & & $\begin{array}{c}\text { Cost } \\
\text { Category } 1 \\
\text { sq.ft }\end{array}$ & \begin{tabular}{|c} 
Cost \\
Category 2 \\
sq.ft
\end{tabular} & \begin{tabular}{|c|} 
Cost \\
Category 3 \\
sq.ft
\end{tabular} & $\begin{array}{c}\text { Cost } \\
\text { Category } 4 \\
\text { sq.ft }\end{array}$ & \\
\hline & Administration Building Subsystem & 12,440 & 3,000 & & & 6,948 \\
\hline & Receiving \& Inspection Subsystem & & 24,227 & 2,970 & 14,839 & 18,916 \\
\hline 3 & Aqueous Waste Treatment Subsystem & & 1,150 & 1,751 & 1,590 & 2,021 \\
\hline 4 & Organic Destruction Subsystem & & 1,128 & 1,717 & 1,560 & 1,983 \\
\hline 5 & Residue \& Sludge Treatment Subsystem & & 2,040 & 3,105 & 2,820 & 3,584 \\
\hline 6 & Soft Debris Treatment Subsystem & & 1,606 & 2,444 & 2,220 & 2,822 \\
\hline 7 & Complex Debris Treatment Subsystem & & & & & \\
\hline 8 & Open Debris Treatment Subsystem & & 1,888 & 2,873 & 2,610 & 3,317 \\
\hline 9 & Bulk Soil Treatment Subsystem & & 1,606 & 2,444 & 2,220 & 2,822 \\
\hline 10 & Metal Decontamination Subsystem & & 641 & 169 & 1,904 & 1,222 \\
\hline 11 & Special Waste & & 604 & 159 & 1,792 & 1,150 \\
\hline 12 & Mercury Amalgamation Subsystem & & 1,020 & 1,552 & 1,410 & 1,792 \\
\hline 13 & Lead Recovery Subsystem & & & & & \\
\hline 14 & Air Pollution Control Subsystem & & 955 & 1,453 & 1,320 & 1,678 \\
\hline 15 & Polymer Stabilization Subsystem & & 3,097 & 8,278 & 5,250 & 7,481 \\
\hline 16 & Certification \& Shipping Subsystem & & 28,277 & & & 12,725 \\
\hline \multirow[t]{3}{*}{17} & Support Subsystem & 3,360 & 16,883 & & & 9,109 \\
\hline & Total Cost & & & & & 77,570 \\
\hline & & \multicolumn{4}{|c|}{ Post Totals To Table 4-3, Item } & 7.0 \\
\hline
\end{tabular}


Table B-3.6 : Total Life-Cycle Cost (without Disposal) Estimate for

Enhanced Integrated Non Thermal Treatment Systems (cost module ENTS-3)

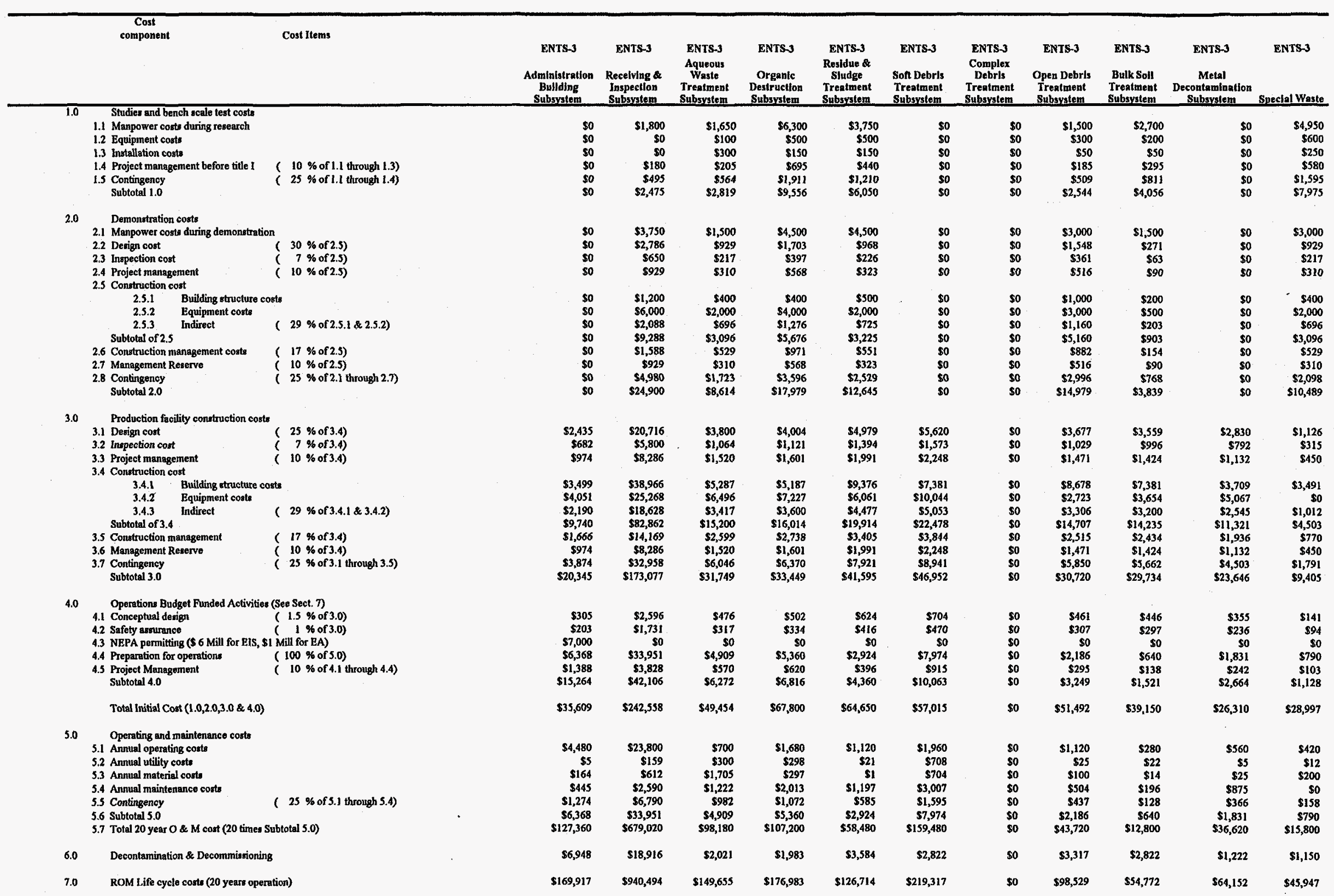


Table B-3.6 : Total Life-Cycle Cost (without Disposal) Estimate for

Enhanced Integrated Non Thermal Treatment Systems (cost module ENTS-3)

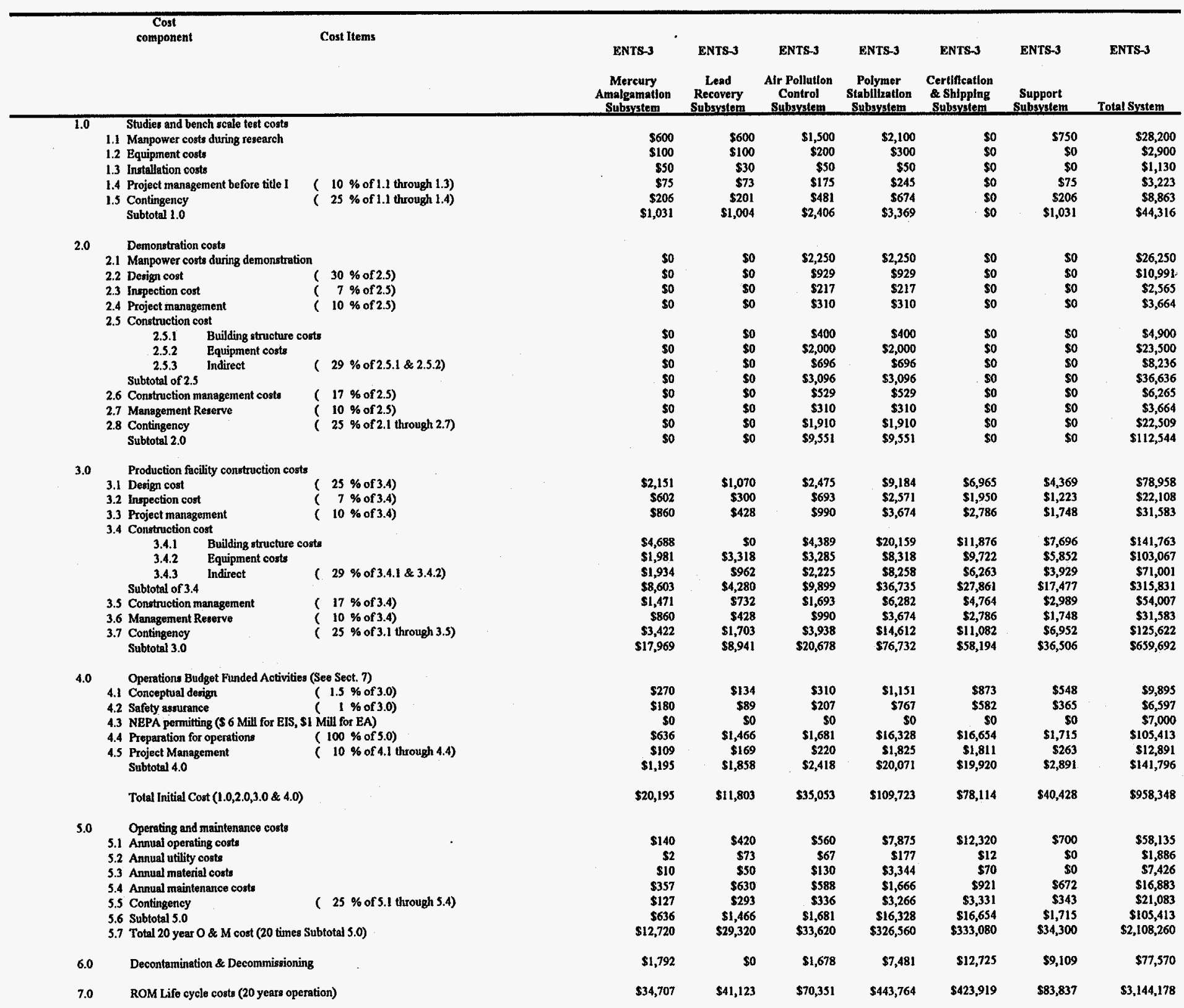




\section{B-4 ENTS-4 Cost Tables}

B-4.1. Equipment Purchase and Installation Budget Estimates

B-4.2. Development, Testing and Evaluation Cost Estimate

B-4.3. Building, Equipment and Installation Cost Summary

B-4.4. Annual Operating and Maintenance Costs

B-4.5. Decontamination and Decommissioning Costs

B-4.6 Total Life-Cycle Cost (without Disposal) Estimate Summary for Enhanced Integrated Nonthermal Treatment Systems 
Table B-4.1: Equipment Purchase and Installation Budget Estimates

Enhanced Non-Thermal Treatment System

ENTS - 4 CWO, Desorption, Compaction and Polymer Stabilization

\begin{tabular}{|c|c|c|c|c|c|c|c|c|}
\hline \multirow{3}{*}{ DESCRIPTION } & \multicolumn{8}{|c|}{$\begin{array}{c}\text { ENTS } 4 \\
\text { CWO, Desorption, Compaction and Polymer Stabilization }\end{array}$} \\
\hline & \multirow{2}{*}{$\begin{array}{l}\text { FAC. } \\
\text { CAT. }\end{array}$} & \multirow[b]{2}{*}{$\mathbf{H P}$} & \multicolumn{3}{|c|}{ MATLS. \& EQUIP. } & \multicolumn{3}{|c|}{ INST. COSTS } \\
\hline & & & QTY & \begin{tabular}{|c|} 
Unit Cost \\
S1000's
\end{tabular} & $\begin{array}{l}\text { Amount } \\
\text { S1000's }\end{array}$ & $\begin{array}{c}\text { Unit Cost } \\
\text { S1000's }\end{array}$ & $\begin{array}{l}\text { Amount } \\
\text { \$1000's }\end{array}$ & $\begin{array}{l}\text { Total } \\
\text { U.O. }\end{array}$ \\
\hline Admwistrition Butding Sulbs stom & Ho & & & & & & & \\
\hline - Administration Office & & & & & & & & \\
\hline - Office Furniture - EMPLOYEE & $E$ & & 49 & 7.0 & 343 & 1.0 & 49 & 392 \\
\hline - Computers & $\underline{E}$ & & Lot & 500.0 & 500 & 50.0 & 50 & 550 \\
\hline - Communication & $\mathbf{E}$ & & Lot & 250.0 & 250 & 35.0 & 35 & 285 \\
\hline - Testing Laboratory & & & & & & & & \\
\hline - ICP-Mass Spectrometer & $\mathrm{D}$ & & 1 & & & & & \\
\hline - Gas Chromatography - Mass Spec. & $\mathrm{D}$ & & 1 & & & & & \\
\hline I- Scintillation Counter - Alpha & D & & 1) & & & & & \\
\hline - Scintillation Counter - Gamma & D & & 1 & & & & & \\
\hline - Geiger -Muller Counter & D & & 1 & & & & & \\
\hline - Fume Hood & $A$ & & 2 & & & & & \\
\hline - Centrifuge & A & & 2 & & & & & \\
\hline - Autoclave & $\mathrm{A}$ & & 1 & & & & & \\
\hline 1- Scrubber/Blower & C & & 1 & & & & & \\
\hline - Hood/HEPA Filtration & $\mathrm{C}$ & & 1 & & & & & \\
\hline - Glovebox & D & & 1 & & & & & \\
\hline - Wet Chemistry Equipment & $\mathrm{D}$ & & 1 & & & & & \\
\hline - Refrigeration/ Storage & A & & 1 & & & & & \\
\hline - Computer \& Data Communication & $\mathrm{D}$ & & 2 & & & & & \\
\hline - Atomic Absorption Spectrography & $\mathrm{D}$ & & 1 & & & & & \\
\hline - Soil / Physical Testing Equipment & $\mathrm{D}$ & & Lot & & & & & \\
\hline - Complete Package per Above Detail & $E$ & & Lot & $1,500.0$ & 1500 & 300.0 & 300 & 1800 \\
\hline - Office Equipment \& Furnishing & $E$ & & Lot & 80.0 & 80 & 7.0 & 7 & 87 \\
\hline - Allowance for Mechanical/Piping & $E$ & & Lot & 26.7 & 26.7 & 132.3 & 132.3 & 159 \\
\hline - Allowance for Electrical/Control & E & & Lot & 401.0 & 401 & 264.6 & 264.6 & 665.6 \\
\hline - Calibration, Testing \& Startup & $E$ & & Lot & 62.0 & 62 & 50.3 & 50.3 & 112.3 \\
\hline Total Administration Building Subsystem & L & & म & & 3162.7 & & 888.2 & 4050.9 \\
\hline 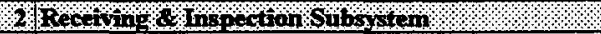 & 1 & & & 8 & 6 & & 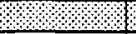 & 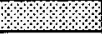 \\
\hline - Unloading, Staging \& Storage (A-RCINS) & & & & & & & & \\
\hline I- O/H Bridge Crane \& Rigging - 20 TN & $\mathbf{E}$ & & 1 & 625.0 & 625 & 125.0 & 125 & 750 \\
\hline - Forklift - $10 \mathrm{TN}$ & $E$ & & 2 & 65.0 & 130 & 10.0 & 20 & 150 \\
\hline - Airlock & $E$ & & I & 124.0 & 124 & 39.0 & 39 & 163 \\
\hline - Inspect \& Assay (A-RCINS) & & & & & & & & \\
\hline - Transfer Cart - 2 TN & $\mathrm{B}$ & & & & & & & \\
\hline - Gamma Spectroscopy (GS) & $\mathrm{D}$ & & & & & & & \\
\hline - Passive Active Neutron Assay (PAN) & D & & & & & & & \\
\hline - Real Time Radiography (RTR) & D & & & & & & & \\
\hline - Load Cell & $\mathrm{D}$ & & & & & & & \\
\hline- CCTV & D & & & & & & & \\
\hline - RHMMS Barrel Monitor including above & E & & 1 & $2,000.0$ & 2000 & 400.0 & 400 & 2400 \\
\hline - Persomnel Contamination Monitor & $E$ & & 1 & 35.0 & 35 & 5.0 & 5 & 40 \\
\hline - Container Decontamination Enclosure & E & & 1) & 140.0 & 140 & 15.0 & 15 & 155 \\
\hline - Open, Dump, \& Sort & & & & & & & & \\
\hline - Self Guided Vehicle & E & & 2 & 425.0 & 850 & 64.0 & 128. & 978 \\
\hline - Decap Device & E & & 3 & 50.0 & 150 & 9.0 & 27 & 177 \\
\hline - Sort Hopper & $\mathbf{E}$ & & 5 & 40.0 & 200 & 4.0 & 20 & 220 \\
\hline - Sort/Rotating Table & E & & 5 & 200.0 & 1000 & 30.0 & 150 & 1150 \\
\hline - Special Waste Glove Box & $E$ & & 1. & 400.0 & 400 & 48.0 & 48 & 448 \\
\hline - Dump Hopper & E & & 6 & 40.0 & 240 & 4.0 & 24 & 264 \\
\hline - Maintenance Crane & E & & 1 & 800.0 & 800 & 120.0 & 120 & 920 \\
\hline Bins & E & & 80 & 5.0 & 400 & 1.0 & 80 & 480 \\
\hline - Bin Conveyor $8 \mathrm{ft}$ long & $E$ & & 20 & 10.0 & 200 & 4.0 & 80 & 280 \\
\hline 1. Bin Conveyor $70 \mathrm{ft}$ long & E & & 2 & 70.0 & 140 & 28.0 & 56 & 196 \\
\hline 1. Bin Pusher & $E$ & & 34 & 10.0 & 340 & 4.0 & 136 & 476 \\
\hline I- Main Cell Gantry Robot & $E$ & & 1 & $2,000.0$ & 2000 & 400.0 & 400 & 2400 \\
\hline - Sorting Table Hydraulic Manipulator & E & & 2 & 120.0 & 240 & 40.0 & 80 & 320 \\
\hline - Sorting Master Slave Manipulator & E & & 5 & 100.0 & 500 & 40.0 & 200 & 700 \\
\hline - Box Size Reduction Unit & $E$ & & 1 & $1,500.0$ & 1500 & 400.0 & 400 & 1900 \\
\hline - Drum Opening Gantry Robot & E & & 2 & 400.0 & 800 & 80.0 & 160 & 960 \\
\hline - Glovebox Hydraulic Manipulator & $E$ & & 2 & 120.0 & 240 & 40.0 & 80 & 320 \\
\hline - Container Grappler & E & & 4 & 50.0 & 200 & 8.0 & 32 & 232 \\
\hline - Decap Enclosure & E & & 3 & 100.0 & 300 & 30.0 & 90 & 390 \\
\hline
\end{tabular}


Table B-4.1: Equipment Purchase and Installation Budget Estimates

Enhanced Non-Thermal Treatment System

ENTS - 4 CWO, Desorption, Compaction and Polymer Stabilization

\begin{tabular}{|c|c|c|c|c|c|c|c|c|}
\hline \multirow{3}{*}{ DESCRIPTION } & \multicolumn{8}{|c|}{$\begin{array}{c}\text { ENTS } 4 \\
\text { CWO, Desorption, Compaction and Polymer Stabilization }\end{array}$} \\
\hline & \multirow{2}{*}{$\begin{array}{l}\text { FAC. } \\
\text { CAT. }\end{array}$} & \multirow[b]{2}{*}{ HP } & \multicolumn{3}{|c|}{ MATLS. \& EQUIP. } & \multicolumn{3}{|c|}{ INST. COSTS } \\
\hline & & & QTY & $\begin{array}{c}\text { Unit Cost } \\
\text { S1000's }\end{array}$ & $\begin{array}{l}\text { Amount } \\
\$ 1000 \text { 's }\end{array}$ & \begin{tabular}{|c|} 
Unit Cost \\
$\$ 1000$ 's
\end{tabular} & $\begin{array}{l}\text { Amount } \\
\text { S1000's }\end{array}$ & $\begin{array}{l}\text { Total } \\
\text { U. } .0 .\end{array}$ \\
\hline - Bin Hoist / Pusher & B & 5 & 1 & 33 & 66 & 8 & 8 & 74 \\
\hline - Discharge. \& Oversize Load Hopper $(100$ CF $)$ & $\bar{B}$ & 1 & 1 & 25 & 50 & 7 & 7 & 57 \\
\hline - Primary Shredder $(25,1.5,2$ tons $/ \mathrm{hr})$ & E & 250 & 1 & 375 & 375 & 75 & 75 & 450 \\
\hline - Secondary Shredder $(.5,1.5,3$ tons $/ \mathrm{hr})$ & E & 60 & 1 & 160 & 160 & 32 & 32 & 192 \\
\hline - Live Bottom Bulk Storage & $B$ & $\mathrm{I}$ & 1 & 25 & 50 & 7 & 7 & 57 \\
\hline - Conveyor / Canister Handler & $B$ & 5 & 1 & 40 & 80 & 10 & 10 & 90 \\
\hline - Dust Collection System w/ Blower & $E$ & & Lot & 108 & 108 & 17 & 17 & 125 \\
\hline - HEPA Filter & $E$ & & 2 & 25 & 50 & 5 & 10 & 60 \\
\hline- CCTV & E & & 8 & 10.0 & 80 & 2.0 & 16 & 96 \\
\hline - Airlack & E & & 4 & 124.0 & 496 & 39.0 & 156 & 652 \\
\hline - Roller up Door (CA) & $E$ & & 2 & 20.0 & 40 & 2.0 & 4 & 44 \\
\hline - Allowance for MechanicallPiping & $\underline{E}$ & & Lot & 755.5 & 755.5 & $1,628.5$ & 1628.5 & 2384 \\
\hline - Allowance for Electrical/Control & E & & Lot & $2,266.4$ & 2266.4 & $1,628.5$ & 1628.5 & 3894.9 \\
\hline - Calibration, Testing \& Startup & $E$ & & Lot & 362.6 & 362.6 & 260.6 & 260.6 & 623.2 \\
\hline Total Receiving \& Inspection Subsystem & \lfloor & & & $\$$ & 18493.5 & & 6774.6 & 25268.1 \\
\hline 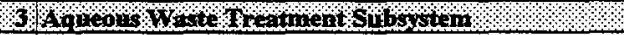 & 11 & & 0 & 10 & २ి & ? & ?ִ & $1 \%$ \\
\hline - Batch Liquid Waste (A-AQWTR) & & & & & & & & \\
\hline - High TOC Batch Tank with Agitator & $\mathbf{E}$ & & 1 & 27.0 & 27 & 5.0 & 5 & 32 \\
\hline - High TOC Batch Tank Pump & $E$ & & 1 & 5.0 & 5 & 1.0 & 1 & 6 \\
\hline - Low TDS Batch Tank with Agitator & $\underline{E}$ & & 1 & 27.0 & 27 & 5.0 & 5 & 32 \\
\hline - Low TDS Batch Tank Pump & $\underline{E}$ & & 1 & 5.0 & 5 & 1.0 & 1 & 6 \\
\hline - High TDS/ Heavy Metals Batch Tank with Agitator & E & & 1 & 27.0 & 27. & 5.0 & 5 & 32 \\
\hline - High TDS/ Heavy Metals Batch Tank Purop & $\underline{E}$ & & 1 & 5.0 & 5 & 1.0 & 1 & 6 \\
\hline - Hg Batch Tank wth Agitator & $\mathbf{E}$ & & 1 & 27.0 & 27 & 5.0 & 5 & 32 \\
\hline - Hg Batch Tank Pump & E & & 1 & 5.0 & 5 & 1.0 & 1 & 6 \\
\hline - Airlocks & E & & 2 & 124.0 & 248 & 39.0 & 78. & 326 \\
\hline- CCTV & E & & 5 & 10.0 & 50 & 2.0 & 10 & 60 \\
\hline - Crane & $\mathbf{E}$ & & 1 & 525.0 & 525 & 105.0 & 105 & 630 \\
\hline - Shield Window & $\mathbf{E}$ & & 1 & 130 & 130 & 44 & 44 & 174 \\
\hline I- Gross Organics Removal (A-AQWTR) & & & & & & & & \\
\hline 1- Oil/ Water Separator & E & & 1 & 18.0 & 18 & 2.5 & 2.5 & 20.5 \\
\hline - Neutralization/Settling (A-AQWTR) & & & & & & & & \\
\hline - Neutralization System & E & & 1 & 95.0 & 95 & 19.0 & 19 & 114 \\
\hline - Neutralization Overflow Pump & $\mathrm{E}$ & & 1 & 5.0 & 5 & 1.0 & 1 & 6 \\
\hline - Sludge Transfer Pump & E & & 1 & 5.0 & 5 & 1.0 & 1 & 6 \\
\hline - Sludge Settler & E & & 1 & 27.0 & 27 & 5.0 & 5 & 32 \\
\hline - Sludge Settlex Pump & E & & 1 & 5.0 & 5 & 1.0 & 1 & 6 \\
\hline - Suspended Solids Filtration (A-AQWTR) & & & & & & & & \\
\hline - Suspended Solids Filter & E & & 1 & 125.0 & 125 & 25.0 & 25 & 150 \\
\hline - Concentration (A-AQWTR) & & & & & & & -1 & \\
\hline - Evaporator with Condenser & E & & 2 & 300.0 & 600 & 75.0 & 150 & 750 \\
\hline - Evaporator Liquids Pump & E & & 2 & 5.0 & 10 & 1.0 & 2 & 12 \\
\hline - Calcium Carbonate Removal & & & & & & & & \\
\hline I- Tank with Agitator & E & & 1 & 27.0 & 27 & 5.0 & 5 & 32 \\
\hline - Transfer Pump & $E$ & & 4 & 5.0 & 20 & 1.0 & 4 & 24 \\
\hline - Decanter Centrifuge & E & & 1 & 450.0 & 450 & 60.0 & 60 & 510 \\
\hline - Holding Tank & $E$ & & 1 & 7.0 & 7 & 1.5 & 1.5 & 8.5 \\
\hline 1. Shurry Pump & $E$ & & 1 & 5.0 & 5 & 1.0 & 1 & 6 \\
\hline Filter & E & & 2 & 2.5 & 5 & 0.5 & 1 & 6 \\
\hline - Dissolved Organies Removal & & & & & & & & \\
\hline - Ozone Generator System & E & & & & & & & \\
\hline - UV/Peroxide System & E & & & & & & & \\
\hline - Ozone Decomposer & $E$ & & & & & & & \\
\hline - Complete Package per Above Detail & $\mathrm{E}$ & & Lot & 125.0 & 125 & 25.0 & 25 & 150 \\
\hline - Liquid-Phase Carbon (55-gal drum) & F & & 4 & 0.8 & 3.2 & 0.1 & 0.4 & 3.6 \\
\hline - Concentrator & 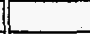 & & & & & & & \\
\hline - Vapor Phase Carbon & $F$ & & 2 & 16.0 & 32 & 3.0 & 6 & 38 \\
\hline - Evaporator with Condenser & E & & 1 & 500.0 & 500 & 75.0 & 75 & 575 \\
\hline - Condenser Liquids Pump & $E$ & & 1 & 5.0 & 5 & 1.0 & 1 & 6 \\
\hline - Dissolved Solids Removal & & & & & & & & \\
\hline - Ion Exchanger & E & & 1 & 61.0 & 61 & 9.0 & 9 & 70 \\
\hline - Treated Water Sampling & & & & & & & & \\
\hline - Treated Water Tanks & E & & 2 & 45.0 & 90 & 12.0 & 24 & 114 \\
\hline
\end{tabular}


Table B-4.1: Equipment Purchase and Installation Budget Estimates

Enhanced Non-Thermal Treatment System

ENTS - 4 CWO, Desorption, Compaction and Polymer Stabilization

\begin{tabular}{|c|c|c|c|c|c|c|c|c|c|}
\hline \multirow{3}{*}{\multicolumn{2}{|c|}{ DESCRIPTION }} & \multicolumn{8}{|c|}{$\begin{array}{c}\text { ENTS 4 } \\
\text { CWO, Desorption, Compaction and Polymer Stabilization }\end{array}$} \\
\hline & & \multirow{2}{*}{$\begin{array}{l}\text { FAC. } \\
\text { CAT. }\end{array}$} & \multirow[b]{2}{*}{$\mathbf{H P}$} & \multirow[b]{2}{*}{ QTY } & \multicolumn{2}{|c|}{ MATLS. \& EQUIP. } & \multicolumn{3}{|c|}{ INST. COSTS } \\
\hline & & & & & $\begin{array}{l}\text { Unit Cost } \\
\text { \$1000's }\end{array}$ & $\begin{array}{l}\text { Amount } \\
\text { S1000's }\end{array}$ & $\begin{array}{l}\text { Unit Cost } \\
\text { S1000's }\end{array}$ & $\begin{array}{l}\text { Amount } \\
\text { S1000's }\end{array}$ & $\begin{array}{l}\text { Total } \\
\text { U.O. }\end{array}$ \\
\hline & - Treated Water Pumps & E & & 2 & \begin{tabular}{|r|}
5.0 \\
\end{tabular} & 10 & 1.0 & 2 & 12 \\
\hline & - Chemical Distribution & & & & & & & & \\
\hline & - Chemical Addition Purmp (Acid) & $E$ & & 1 & 5.0 & 5 & 1.0 & 1 & 6 \\
\hline & - Chemical Addition Pump (Caustic) & E & & 1 & 5.0 & 5 & 1.0 & 1 & 6 \\
\hline & - Chemical Addition Pump (Sulfide) & E & & 1 & 5.0 & 5 & 1.0 & 1 & 6 \\
\hline & - Chemical Addition Pump (Floc/ Polymer) & $E$ & & 1 & 1.0 & 1 & 0.3 & 0.3 & 1.3 \\
\hline & - Chemical Storage Tanks & $\underline{E}$ & & 4 & 7.0 & 28 & 1.5 & 6 & 34 \\
\hline & - Chemical Addition Pump (Peroxide) & E & & 1 & 5.0 & 5 & 1.0 & 1 & 6 \\
\hline & - Peroxide Storage Tank & E & & 1 & 7.0 & 7 & 1.5 & 1.5 & 8.5 \\
\hline & - Allowance for Mechanical/Piping & E & & Lot & $1,010.2$ & 1010.2 & 416.5 & 416.5 & 1426.7 \\
\hline & - Allowance for Electrical/Control & $\underline{E}$ & & Lot & 505.1 & 505.1 & 347.1 & 347.1 & 852.2 \\
\hline & Calibration, Testing \& Startup & $E$ & & Lot & 97.7 & 97.7 & 58.3 & 58.3 & 156 \\
\hline & Total Aqueous Waste Treatment Subsystem & 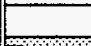 & & & & 4980.2 & & 1516.1 & 6496.3 \\
\hline 4 & Orspanc D estruction Subssutem & itis & 8 & $10 \%$ & 1. & 10,0 & 12 & 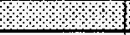 & $1+$ \\
\hline & - Prepare and Feed Incoming Waste & & & & & & & & \\
\hline & - Feed Conveyor Lift/ Hoist/ Bins & $E$ & & Lot & 50 & 50 & 8 & 8 & 58 \\
\hline & - Discharge and Loading Hoppers & $\underline{E}$ & & Lot & 45 & 45 & 8 & 8 & 53 \\
\hline & Feed Blender & $E$ & & Lot & 45 & 45 & 8 & 8 & 53 \\
\hline & - Detox Process & & & & & & & & \\
\hline & - Reaction Vessel & $E$ & & & & & & & \\
\hline & - Reflux Condenser & $E$ & & & & & & & \\
\hline & - Filter & $E$ & & & & & & & \\
\hline & - Solids Feeder & E & & & & & & & \\
\hline & - Solution Cooler & $\underline{\mathbf{E}}$ & & & & & & & \\
\hline & - Overhead Condenser & $E$ & & & & & & & \\
\hline & Pumps & $\mathbf{E}$ & & & & & & & \\
\hline & - Condensate Catch & E & & & & & & & \\
\hline & - Neutralization Tanks & E & & & & & & & \\
\hline & - Filtered Solids Rinse Tanks & $E$ & & & & & & & \\
\hline & - Oil Heating and Cooling System & $E$ & & & & & & & \\
\hline & - Total Cost For Above Package & $\mathbf{E}$ & & Lot & 2400 & 2400 & 300 & 300 & 2700 \\
\hline & - Miscellaneous & 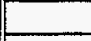 & & & & & & & \\
\hline & - Neutralization System & E & & 1 & 95 & 95 & 19 & 19 & 114 \\
\hline & - Neutralization Overflow Pump & $\underline{E}$ & & 1 & 5 & 5 & 1 & 1 & 6 \\
\hline & - Sludge Transfer Pump & E & & 1 & 5 & 5 & 1 & 1 & 6 \\
\hline & - Crane & E & & 1 & 525 & 525 & 105 & 105 & 630 \\
\hline & - CCTV & E & & 8 & 10 & 80 & 2 & 16 & 96 \\
\hline & Airlock & E & & 2 & 124 & 248 & 39 & 78 & 326 \\
\hline & - Shield Window & E & & 3 & 130 & 390 & 44 & 132 & 522 \\
\hline & - Allowance for Mechanical/Piping & $\mathbf{E}$ & & Lot & $1,166.4$ & 1166.4 & 405.6 & 405.6 & 1572 \\
\hline & - Allowance for Electrical/Control & E & & Lot & 583.2 & 583.2 & 338.0 & 338 & 921.2 \\
\hline & Calibration, Testing \& Startup & $E$ & & Lot & 112.8 & 112.8 & 56.8 & 56.8 & 169.6 \\
\hline & Total Organic Destruction Subsystem & & & & & 5750.4 & 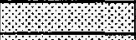 & 1476.4 & 7226.8 \\
\hline $\mathbf{s}$ & 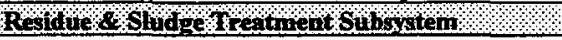 & II: & & 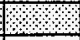 & , & 1,0 & 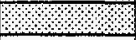 & 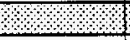 & ?+: \\
\hline & - Feed Preparation and Size Reduction & & & & & & & & \\
\hline & - Feed Conveyor Lif/Hoist/Bins & E & & 1 & 50 & 50 & 8 & 8 & 58 \\
\hline & - Discharge. \& Oversize Load Hopper $(100$ CF $)$ & $\mathbf{E}$ & & 1 & 45 & 45 & 8 & 8 & 53 \\
\hline & Manipulate Crane $10 \mathrm{TN}$ & $E$ & 15 & 1 & 525 & 525 & 105 & 105 & 630 \\
\hline & Shield Window & $E$ & & 1 & 130.0 & 130 & 44.0 & 44 & 174 \\
\hline & - Airlock & E & & 2 & 124 & 248 & 39 & 78 & 326 \\
\hline & - CCTV & $E$ & & 10 & 10 & 100 & 2 & 20 & 120 \\
\hline & - Feed Blender & E & & 1 & 45 & 45 & 8 & 8 & 53 \\
\hline & - Dewatering System & $\mathrm{E}$ & & 1 & & & & & \\
\hline & - THERMAL DESORPTION & & & & & & & & \\
\hline & - High Vacuum Dryer & & & 1 & & & & & \\
\hline & - Nitrogen gas storage and feed tank & E & & 1 & & & & & \\
\hline & - Vacumm Pump & $\mathbf{E}$ & & 1 & & & & & \\
\hline & Filter & E & & 1 & & & & & \\
\hline & - Hot Oil System & & & & & & & & \\
\hline & - Oil Storage and Feed Tank & E & & 1 & & & & & \\
\hline & - Oil Heater & $\mathbf{E}$ & & 1 & & & & & \\
\hline & - Fuel Storage and Feed Tank & $\mathbf{E}$ & & 1 & & & & & \\
\hline
\end{tabular}


Table B-4.1: Equipment Purchase and Installation Budget Estimates

Enhanced Non-Thermal Treatment System

ENTS - 4 CWO, Desorption, Compaction and Polymer Stabilization

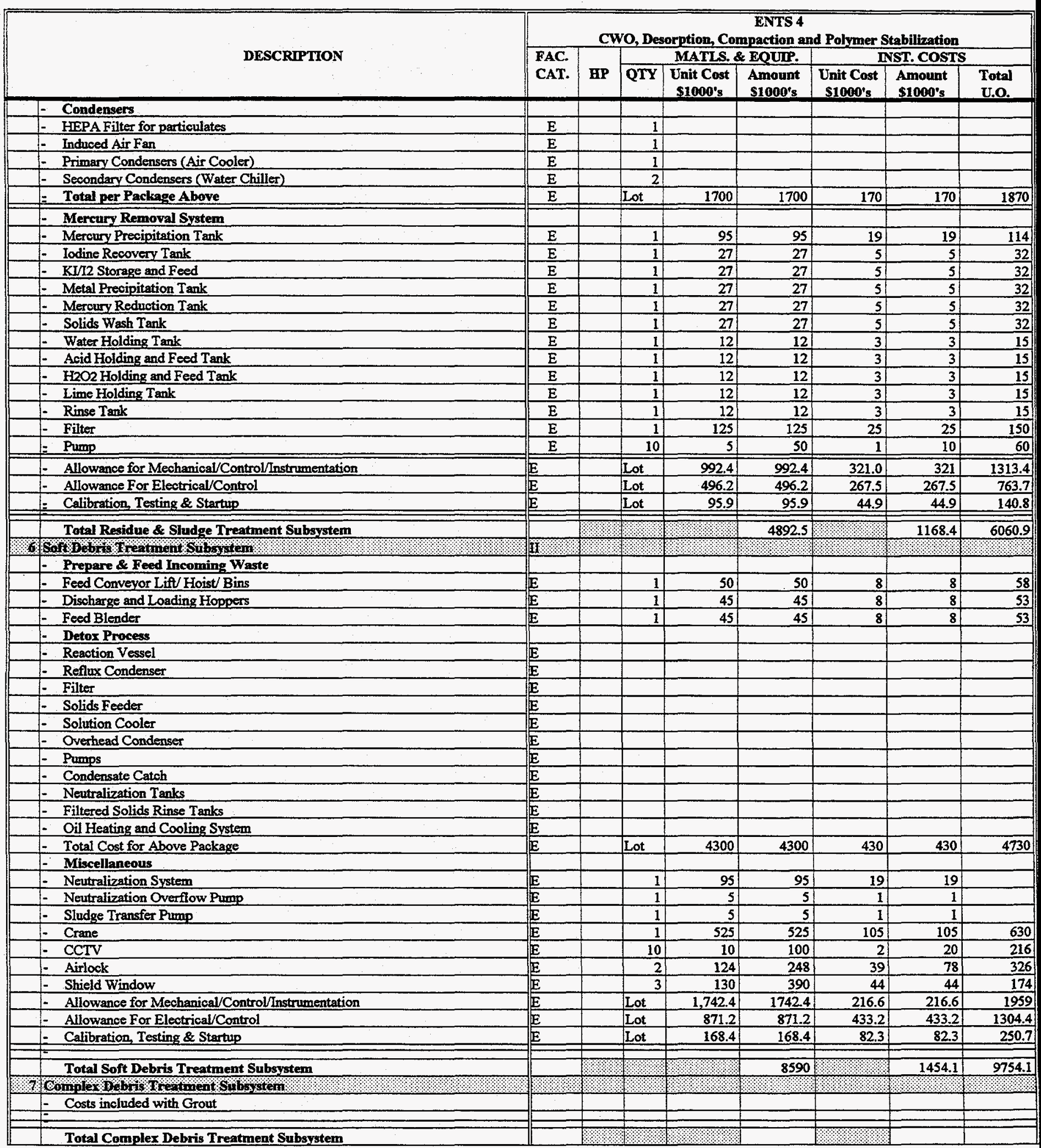


Table B-4.1: Equipment Purchase and Installation Budget Estimates Enhanced Non-Thermal Treatment System ENTS - 4 CWO, Desorption, Compaction and Polymer Stabilization

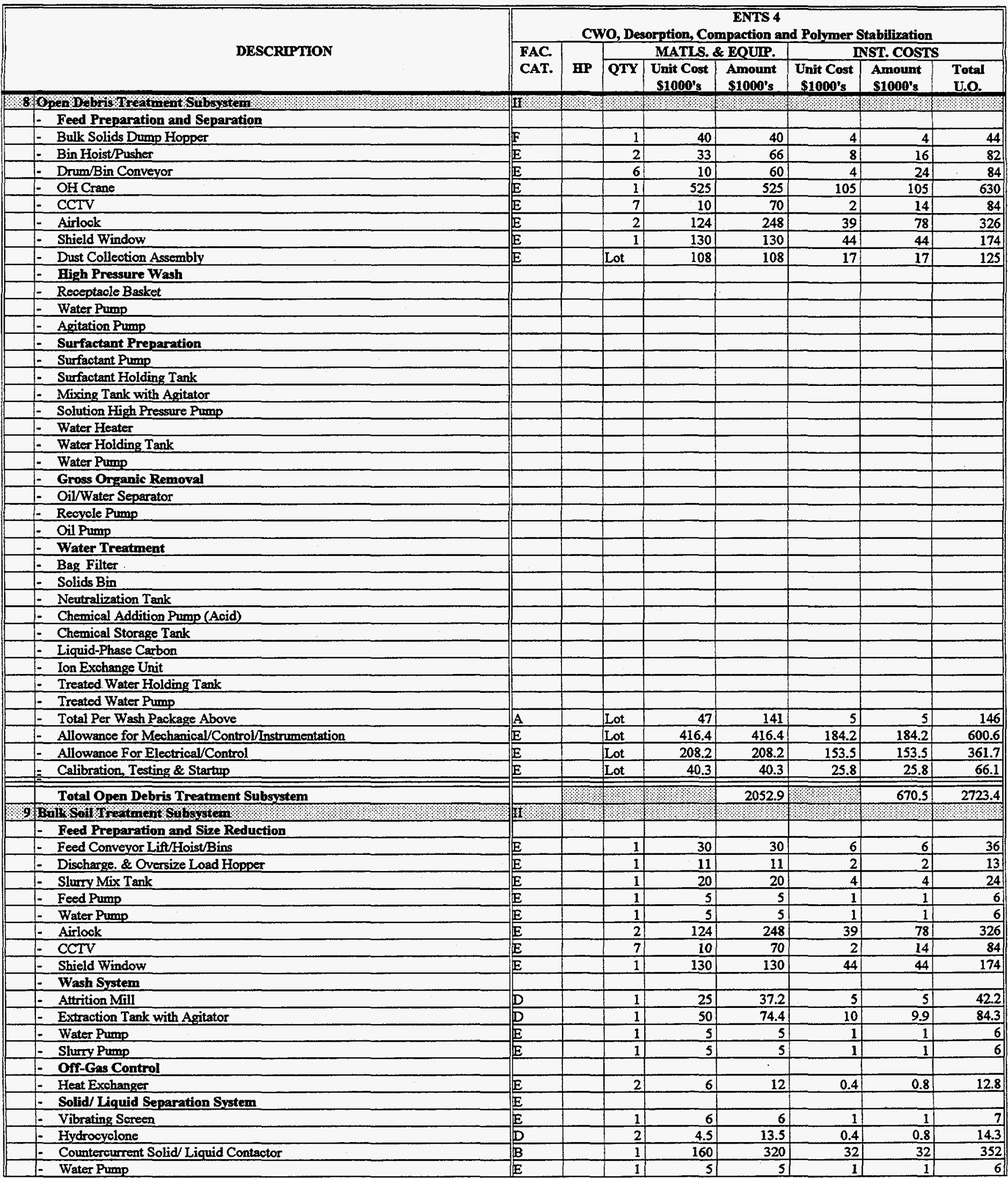


Table B-4.1: Equipment Purchase and Installation Budget Estimates Enhanced Non-Thermal Treatment System ENTS - 4 CWO, Desorption, Compaction and Polymer Stabilization

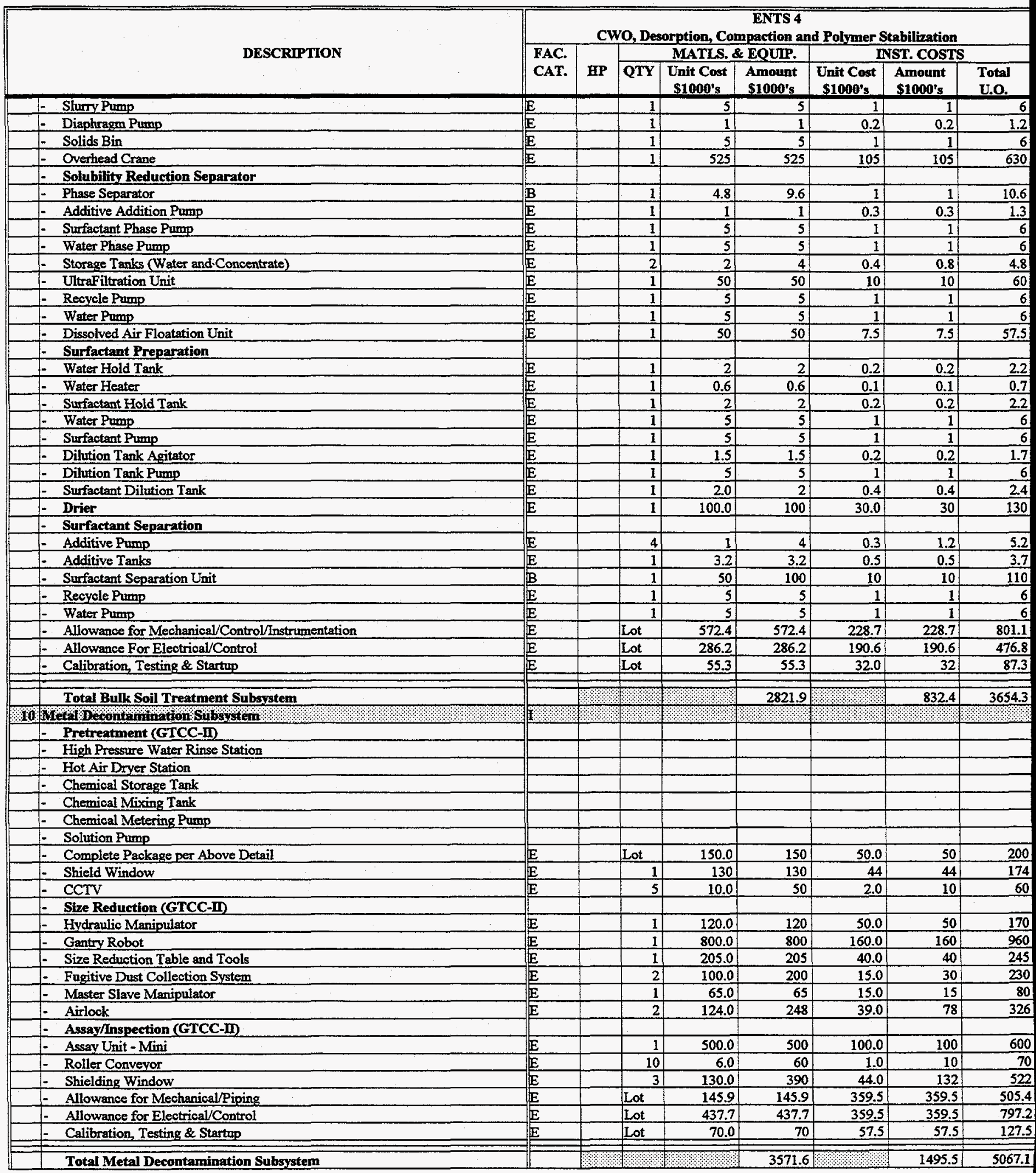


Table B-4.1: Equipment Purchase and Installation Budget Estimates

Enhanced Non-Thermal Treatment System

ENTS - 4 CWO, Desorption, Compaction and Polymer Stabilization

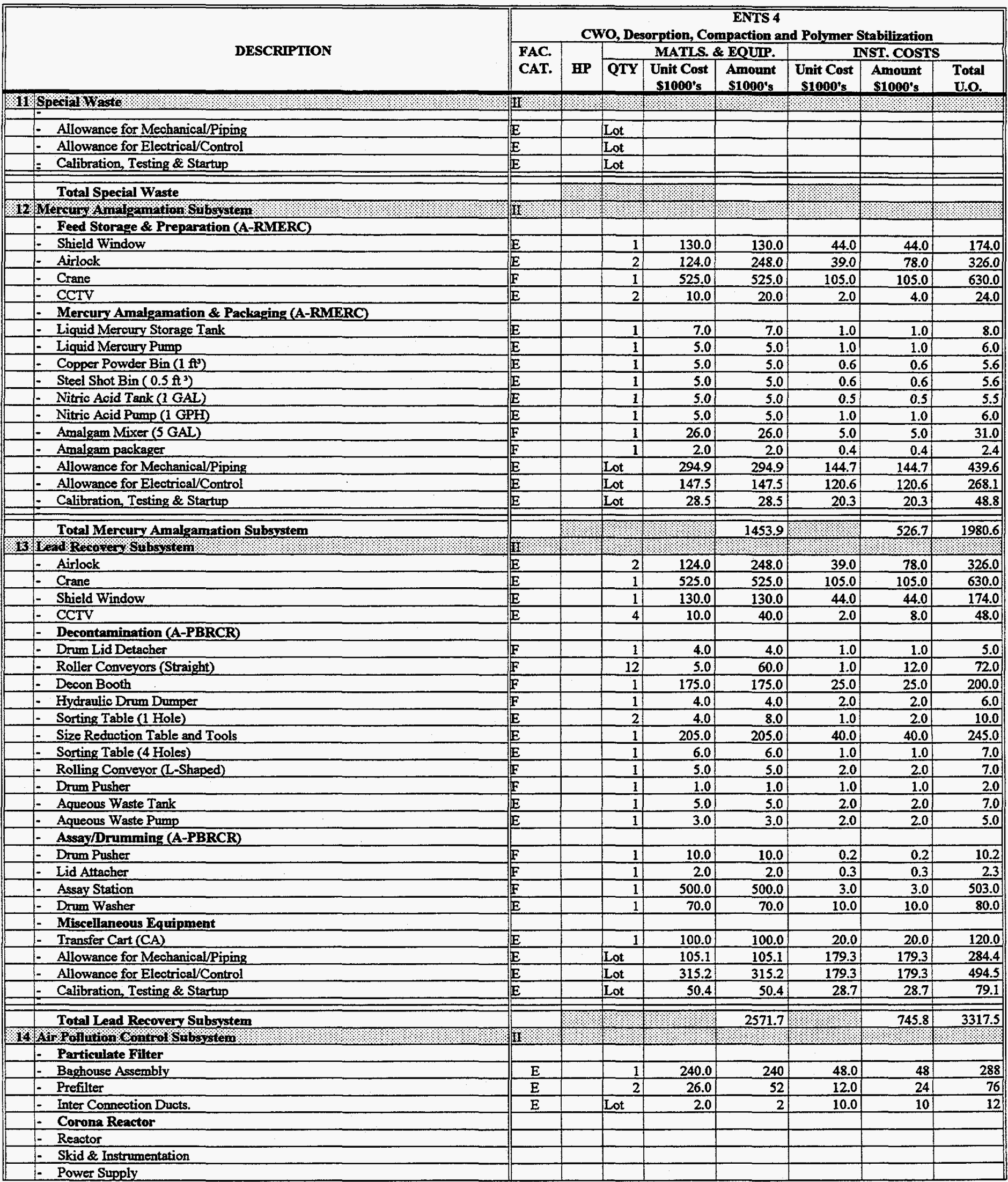


Table B-4.1: Equipment Purchase and Installation Budget Estimates Enhanced Non-Thermal Treatment System ENTS - 4 CWO, Desorption, Compaction and Polymer Stabilization

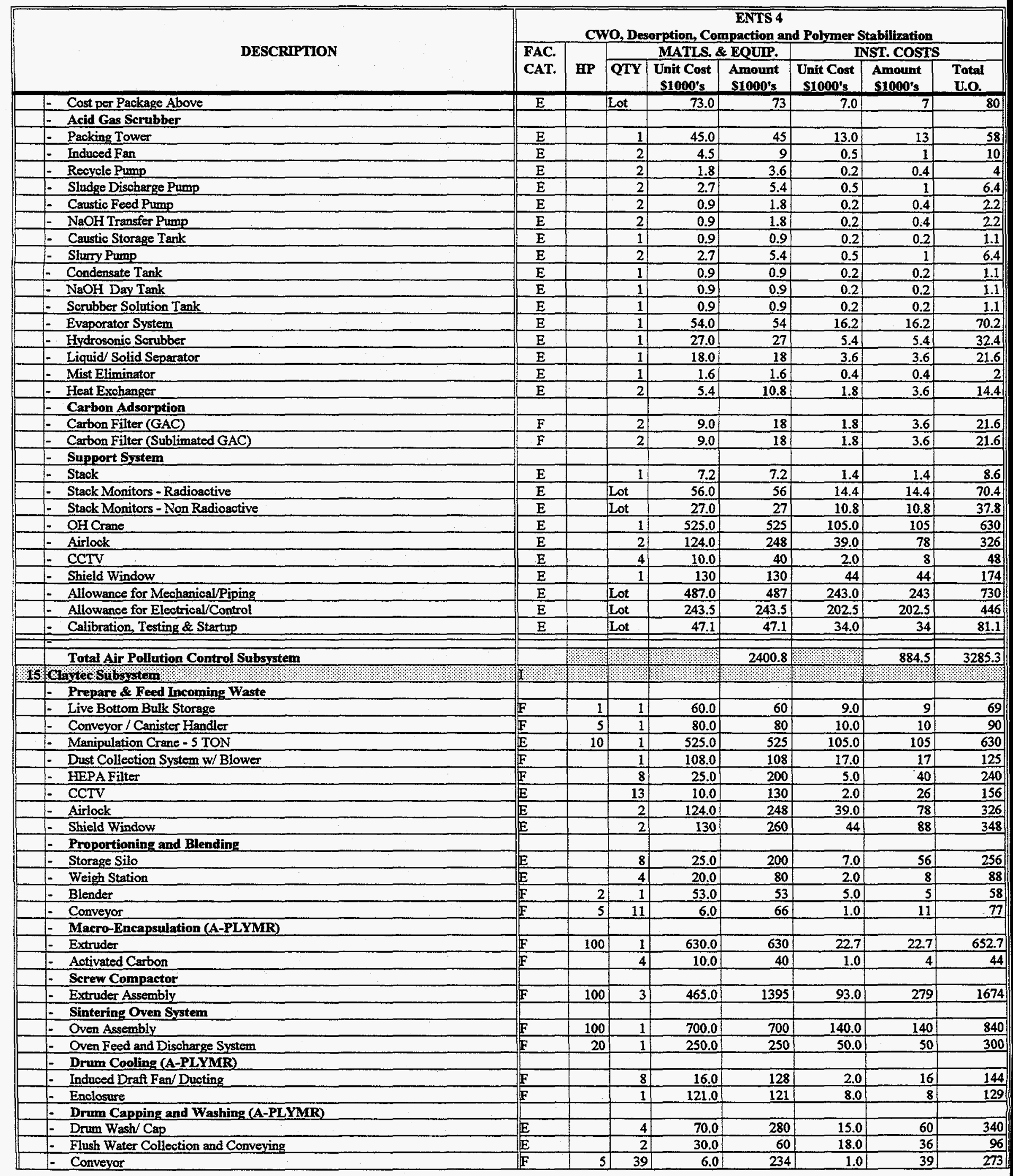


Table B-4.1: Equipment Purchase and Installation Budget Estimates

Enhanced Non-Thermal Treatment System

ENTS - 4 CWO, Desorption, Compaction and Polymer Stabilization

\begin{tabular}{|c|c|c|c|c|c|c|c|c|c|}
\hline \multirow{3}{*}{\multicolumn{2}{|c|}{ DESCRIPTION }} & \multicolumn{8}{|c|}{$\begin{array}{c}\text { ENTS } 4 \\
\text { CWO, Desorption, Compaction and Polymer Stabilization }\end{array}$} \\
\hline & & \multirow{2}{*}{$\begin{array}{l}\text { FAC. } \\
\text { CAT. }\end{array}$} & \multirow[b]{2}{*}{ HP } & \multicolumn{3}{|c|}{ MATLS. \& EQUIP. } & \multicolumn{3}{|c|}{ INST. COSTS } \\
\hline & & & & QTY & \begin{tabular}{|c|} 
Unit Cost \\
\$1000's \\
\end{tabular} & $\begin{array}{l}\text { Amount } \\
\text { \$1000's }\end{array}$ & $\begin{array}{c}\text { Unit Cost } \\
\text { \$1000's }\end{array}$ & $\begin{array}{l}\text { Amount } \\
\text { S1000's }\end{array}$ & $\begin{array}{l}\text { Total } \\
\text { U.O. }\end{array}$ \\
\hline & - Allowance for Mechanical/Piping & $E$ & & Lot & 292.4 & 292.4 & 553.9 & 553.9 & 846.3 \\
\hline & - Allowance for Electrical/Control & $E$ & & Lot & 877.2 & 877.2 & 553.9 & 553.9 & 1431.1 \\
\hline & - Calibration, Testing \& Startup & E & & Lot & 140.4 & 140.4 & 88.6 & 88.6 & 229 \\
\hline & Total Claytec Subsystem & & & & & 7158 & & 2304.1 & 9462.1 \\
\hline & Certificition $\alpha /$ Shing in $\mathrm{Subs}$ stem & 霉 & & 16 & $1 \%$ & ৮/ & ? & & \\
\hline & - Incoming Material Storage (B-CSHIP) & & & & & & & & \\
\hline & - Overhead Bridge Crane $20 \mathrm{TN}$ & $E$ & & 1 & 625.0 & 625 & 125.0 & 125 & 750 \\
\hline & Forklift $10 \mathrm{TN}$ & E & & 1 & 65.0 & 65 & 10.0 & 10 & 75 \\
\hline & - Inspect \& Assay (B-CSHIP) & & & & & & & & \\
\hline & - Transfer Cart 2 TN & E & & & & & & & \\
\hline & - Real Time Radiography (ND) & & & & & & & & \\
\hline & - Passive Active Neutron (ND) & & & & & & & & \\
\hline & - Segmented Gamma Spectroscopy (ND) & & & & & & & & \\
\hline & - Complete Package per Above & E & & 3 & $1,500.0$ & 4500 & 150.0 & 450 & 4950 \\
\hline & - Self Guided Vehicle (ND) & $\mathrm{E}$ & & 1 & 425.0 & 425 & 850.0 & 850 & 1275 \\
\hline & - Truck Loading (B-CSHIP) & & & & & & & & \\
\hline & Forklift $10 \mathrm{TN}$ & $E$ & & 10 & 65.0 & 650 & 10.0 & 100 & 750 \\
\hline & - Roll-up Door & E & & 2 & 20.0 & 40 & 2.0 & 4 & 44 \\
\hline & - Bridge Crane & E & & 1 & 525.0 & 525 & 105.0 & 105 & 630 \\
\hline & - Roller Conveyor (CA) & E & & 20 & 2.0 & 40 & 1.0 & 20 & 60 \\
\hline & - Allowance for Mechanical/Piping & $E$ & & Lot & 343.5 & 343.5 & 832.0 & 832 & 1175.5 \\
\hline & - Allowance for Electrical/Control & $E$ & & Lot & $1,030.5$ & 1030.5 & 832.0 & 832 & 1862.5 \\
\hline & - Calibration. Testing \& Startup & $E$ & & Lot & 164.9 & 164.9 & 133.1 & 133.1 & 298 \\
\hline & Total Certification \& Shipping Subsystem & & & & & 8408.9 & & 3461.1 & 11870 \\
\hline & 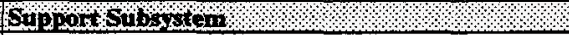 & 俩 & & ? & 3 & & ?+: & ? & 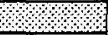 \\
\hline & - Electrical Distribution and MCC & & & & & & & & \\
\hline & - Control Panel - FT & E & & 110 & 10.0 & 1100 & 2.0 & 220 & 1320 \\
\hline & - Emergency Power Generator & $E$ & & 1 & 500.0 & 500 & 100.0 & 100 & 600 \\
\hline & - Main Control Panel & $E$ & & 20 & 10.0 & 200 & 2.0 & 40 & 240 \\
\hline & - Motor Control Center & $E$ & & 100 & 1.5 & 150 & 0.5 & 50 & 200 \\
\hline & - Stand by Emergency Power & E & & 1 & 230.0 & 230 & 80.0 & 80 & 310 \\
\hline & - Utilities and Mechanical & & & & & & & & \\
\hline & - Breathing Air Package & $E$ & & Lot & 70.0 & 70 & 30.0 & 30 & 100 \\
\hline & - Compressed Air Package & $E$ & & Lot & 100.0 & 100 & 25.0 & 25 & 125 \\
\hline & - Instrument Air & E & & 1 & 70.0 & 70 & 30.0 & 30 & 100 \\
\hline & - Instrument Air Package & $\mathbf{E}$ & & Lot & 90.0 & 90 & 25.0 & 25 & 115 \\
\hline & - Plant Steam/Boiler & $E$ & & 1 & 75.0 & 75 & 25.0 & 25 & 100 \\
\hline & - Service Water & E & & 1 & 130.0 & 130 & 40.0 & 40 & 170 \\
\hline & - Sump Pump & E & 3 & 10 & 5.0 & so & 2.0 & 20 & 70 \\
\hline & - Cooling Tower & E & & 1 & 200.0 & 200 & 10.0 & 10 & 210 \\
\hline & - Cooling Water Pumps & E & 3 & 2 & 15.0 & 30 & 3.0 & 6 & 36 \\
\hline & 1- Heating, Ventilation \& Exhaust & & & & & & & & \\
\hline & - Included with Building Cost & & & & & & & & \\
\hline & - Radiation Monitoring & & & & & & & & \\
\hline & - Air Monitors & E & & 44 & 10.0 & 440 & 2.0 & 88 & 528 \\
\hline & - Area Monitoring & E & & 44 & 4.5 & 198 & 1.0 & 44 & 242 \\
\hline & - Personnel Monitoring Portals & E & & 6 & 35.0 & 210 & 5.0 & 30 & 240 \\
\hline & - Portal Monitors & $E$ & & 6 & 35.0 & 210 & 5.0 & 30 & 240 \\
\hline & - Allowance for Mechanical/Piping & E & & Lot & 40.5 & 40.5 & 8.9 & 8.9 & 49.4 \\
\hline & - Allowance for Electrical/Control & $E$ & & Lot & 608.0 & 608 & 134.0 & 134 & 742 \\
\hline & - Calibration, Testing \& Startup & E & & Lot & 94.0 & 94 & 20.7 & 20.7 & 114.7 \\
\hline & Total Support Subsystem & ] & 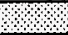 & & & 4795.5 & & 1056.6 & 5852.1 \\
\hline & \% & & & & & & & \%ి & \\
\hline
\end{tabular}


Table B-4.2 : Development, Testing \& Evaluation Cost Estimates

ENTS - 4 CWO, Desorption, Compaction and Polymer Stabilization

\begin{tabular}{|c|c|c|c|c|c|c|c|c|c|c|}
\hline & \multirow[b]{2}{*}{ UNIT OPERATION } & \multicolumn{9}{|c|}{$\begin{array}{c}\text { ENTS - } \\
\text { CWO, Desorption, Compaction and Polymer Stabilization }\end{array}$} \\
\hline & & $\begin{array}{c}\text { Primary } \\
\text { Paper } \\
\text { Evaluation } \\
\text { Research } \\
\text { Manpower }\end{array}$ & $\begin{array}{c}\text { Bench } \\
\text { Scale } \\
\text { Studies } \\
\text { Research } \\
\text { Manpower } \\
\end{array}$ & $\begin{array}{c}\text { Sec. } \\
\text { Paper } \\
\text { Evaluation } \\
\text { Research } \\
\text { Manpower } \\
\end{array}$ & $\begin{array}{c}\text { Total } \\
\text { Research } \\
\text { Manpower }\end{array}$ & \begin{tabular}{|c|} 
Bench \\
Scale \\
Studies \\
Equipment \\
$\mathbf{S 1 0 0 0}$ \\
\end{tabular} & $\begin{array}{c}\text { Bench } \\
\text { Scale } \\
\text { Studies } \\
\text { Installation } \\
\mathbf{S 1 0 0 0} \\
\end{array}$ & $\begin{array}{c}\text { Pilot } \\
\text { Plant } \\
\text { Mock-up } \\
\text { Test Demo. } \\
\text { Manpower } \\
\end{array}$ & $\begin{array}{c}\text { Pilot } \\
\text { Plant } \\
\text { Mock-up } \\
\text { Test Bldg. } \\
\$ 1000 \\
\end{array}$ & \begin{tabular}{|c|} 
Pilot \\
Plant \\
Mock-up \\
Test Equip. \\
$\mathbf{S 1 0 0 0}$ \\
\end{tabular} \\
\hline 1 & Administration Building Subsystern & & & & & & & & & \\
\hline 2 & Receiving \& Inspection Subsystem & 12 & & & 12 & & & 25 & 1,200 & 6,000 \\
\hline 3 & Aqueous Waste Treatment Subsystem & 5 & 3 & 3 & 11 & 100 & 300 & 10 & 400 & 2,000 \\
\hline 4 & Organic Destruction Subsystem & 18 & 20 & 4 & 42 & 500 & 150 & 30 & 400 & 4,000 \\
\hline 5 & Residue \& Sludge Treatment Subsystem & 13 & 8 & 4 & 25 & 500 & 150 & 30 & 500 & 2,000 \\
\hline 6 & Soft Debris Treatment Subsystem & & & & & & & & & \\
\hline 7 & Complex Debris Treatment Subsystem & & & & & & & & & \\
\hline 8 & Open Debris Treatment Subsystem & 2 & 6 & 2 & 10 & 300 & 50 & 20 & 1,000 & 3,000 \\
\hline 9 & Bulk Soil Treatment Subsystem & 10 & 6 & 2 & 18 & 200 & 50 & 10 & 200 & 500 \\
\hline 10 & Metal Decontamination Subsystem & & & & & & & & & \\
\hline 11 & Special Waste & 8 & 20 & 5 & 33 & 600 & 250 & 20 & 400 & 2,000 \\
\hline 12 & Mercury Amalgamation Subsystem & 1 & 3 & & 4 & 100 & 50 & & & \\
\hline 13 & Lead Recovery Subsystem & 2 & 2 & & 4 & 100 & 30 & & & \\
\hline 14 & Air Pollution Control Subsystem & 5 & 3 & 2 & 10 & 200 & 50 & 15 & 400 & 2,000 \\
\hline 15 & Claytec Subsystem & 2 & 8 & 4 & 14 & 300 & 50 & 15 & 400 & 2,000 \\
\hline 16 & Certification \& Shipping Subsystem & & & & & & & & & \\
\hline 17 & Support Subsystem & 5) & & & 5 & & & & & \\
\hline & Unit Cost (\$/Unit) & & & & $\$ 150,000$ & & & $\$ 150,000$ & . & \\
\hline & Total Cost & & & & 28,200 & 2,900 & 1,130 & 26,250 & 4,900 & 23,500 \\
\hline & & Post Totals To T & able 4-4, Item & & 1.1 & 1.2 & 1.3 & 2.1 & 2.5 .1 & 2.5 .2 \\
\hline
\end{tabular}


Table B-4.3 : Building, Equipment and Installation Cost Summary

ENTS - 4 CWO, Desorption, Compaction and Polymer Stabilization

\begin{tabular}{|c|c|c|c|c|c|c|c|c|c|c|}
\hline & \multirow{3}{*}{ UNIT OPERATION } & \multicolumn{9}{|c|}{$\begin{array}{c}\text { ENTS - } \\
\text { CWO, Desorption, Compaction and Polymer Stabilization }\end{array}$} \\
\hline & & \multicolumn{5}{|c|}{ Building Area } & \multicolumn{3}{|c|}{ Material \& Equipment Costs } & \multirow{2}{*}{$\begin{array}{c}\text { Total } \\
\text { Cost per } \\
\text { Unit Oprtn. } \\
\$ 1000\end{array}$} \\
\hline & & $\begin{array}{l}\text { Cost } \\
\text { Category } 1 \\
\text { sq.ft } \\
\end{array}$ & $\begin{array}{l}\text { Cost } \\
\text { Category } 2 \\
\quad \text { sq.ft } \\
\end{array}$ & $\begin{array}{l}\text { Cost } \\
\text { Category } 3 \\
\quad \text { sq.ft } \\
\end{array}$ & $\begin{array}{l}\text { Cost } \\
\text { Category } 4 \\
\text { sg.ft }\end{array}$ & $\begin{array}{c}\text { Total } \\
\text { Area Cost } \\
\$ 1000\end{array}$ & $\begin{array}{c}\begin{array}{c}\text { Purchase } \\
\text { Cost } \\
\$ 1000\end{array} \\
\end{array}$ & $\begin{array}{c}\text { Installation } \\
\text { Cost } \\
\$ 1000 \\
\end{array}$ & $\begin{array}{c}\text { Total } \\
\text { Cost } \\
\$ 1000\end{array}$ & \\
\hline 1 & Administration Building Subsystem & 12440 & 3,000 & & & 3499 & 3162.7 & 888.2 & 4051 & 7550 \\
\hline 2 & Receiving \& Inspection Subsystem & & 24,227 & 2,970 & 14,839 & 38966 & 18493.5 & 6774.6 & 25268 & 64234 \\
\hline 3 & Aqueous Waste Treatment Subsystem & & 1,150 & 1,751 & 1,590 & 5287 & 4980.2 & 1516.1 & 6496 & 11783 \\
\hline 4 & Organic Destruction Subsystem & & 1,128 & 1,717 & 1,560 & 5187 & 5750.4 & 1476.4 & 7227 & 12414 \\
\hline 5 & Residue \& Sludge Treatment Subsystem & & 2,040 & 3,105 & 2,820 & 9376 & 4892.5 & 1168.4 & 6061 & 15437 \\
\hline 6 & Soft Debris Treatment Subsystem & & 1,606 & 2,444 & 2,220 & 7381 & 8590 & 1454.1 & 10044 & 17425 \\
\hline 7 & Complex Debris Treatment Subsystem & $=$ & & & & & & & & \\
\hline 8 & Open Debris Treatment Subsystem & & 1,888 & 2,873 & 2,610 & 8678 & 2052.9 & 670.5 & 2723 & 11401 \\
\hline 9 & Bulk Soil Treatment Subsystem & & 1,606 & 2,444 & 2,220 & 7381 & 2821.9 & 832.4 & 3654 & 11035 \\
\hline 10 & Metal Decontamination Subsystem & & 641 & 169 & 1904 & 3709 & 3571.6 & 1495.5 & 5067 & 8776 \\
\hline 11 & Special Waste & & 604 & 159 & 1792 & 3491 & & & & 3491 \\
\hline 12 & Mercury Amalgamation Subsystem & & 1020 & 1552 & 1410 & 4688 & 1453.9 & 526.7 & 1981 & 6669 \\
\hline 13 & Lead Recovery Subsystem & & & & & & 2571.7 & 745.8 & 3318 & 3318 \\
\hline 14 & Air Pollution Control Subsystem & & 955 & 1,453 & 1,320 & 4389 & 2400.8 & 884.5 & 3285 & 7674 \\
\hline 15 & Claytec Subsystem & & 3,097 & 8,278 & 5,250 & 20159 & 7158 & 2304.1 & 9462 & 29621 \\
\hline 16 & Certification \& Shipping Subsystem & & 28,277 & & & 11876 & 8408.9 & 3461.1 & 11870 & 23746 \\
\hline \multirow[t]{3}{*}{17} & Support Subsystem & 3360 & 16,883 & & & 7696 & 4795.5 & 1056.6 & 5852 & 13548 \\
\hline & Total Cost & \multirow{2}{*}{\multicolumn{4}{|c|}{ Post Totals To Table 4-4, Item }} & 141,763 & & & 106,359 & 248,122 \\
\hline & & & & & & 3.4 .1 & & & 3.4 .2 & \\
\hline
\end{tabular}




\begin{tabular}{|c|c|c|c|c|c|c|c|c|}
\hline & \multirow[b]{2}{*}{ UNIT OPERATION } & \multicolumn{7}{|c|}{$\begin{array}{c}\text { ENTS - } 4 \\
\text { CWO, Desorption, Compaction and Polymer Stabilization }\end{array}$} \\
\hline & & $\begin{array}{l}\text { Operating } \\
\text { FTE }\end{array}$ & $\begin{array}{l}\text { Utilities } \\
\$ 1000 \\
\end{array}$ & \begin{tabular}{c|} 
Materials \\
$\$ 1000$ \\
\end{tabular} & \begin{tabular}{|c|} 
Maintenance \\
Labor (1) \\
$\$ 1000$
\end{tabular} & $\$ 1000$ & $\begin{array}{c}\text { Maintenance } \\
\text { Materials (2) } \\
\$ 1000 \\
\end{array}$ & $\begin{array}{l}\text { Totals } \\
\$ 1000 \\
\end{array}$ \\
\hline 1 & Administration Building Subsystem & 32 & 5 & 164 & 318 & 3162.7 & 127 & 445 \\
\hline 2 & Receiving \& Inspection Subsystem & 170 & 159 & 612 & 1,850 & 18493.5 & 740 & 2,590 \\
\hline 3 & Aqueous Waste Treatment Subsystem & 5 & 300 & 1,705 & 873 & 4980.2 & 349 & 1,222 \\
\hline 4 & Organic Destruction Subsystem & 12 & 298 & 297 & 1,438 & 5750.4 & 575 & 2,013 \\
\hline 5 & Residue \& Sludge Treatment Subsystem & 8 & 21 & 1 & 855 & 4892.5 & 342 & 1,197 \\
\hline 6 & Soft Debris Treatment Subsystem & 14 & 708 & 704 & 2,148 & 8590 & 859 & 3,007 \\
\hline 7 & Complex Debris Treatment Subsystem & & & & & & & \\
\hline 8 & Open Debris Treatment Subsystem & 8 & 25 & 100 & 360 & 2052.9 & 144 & 504 \\
\hline 9 & Bulk Soil Treatment Subsystem & 2 & 22 & 14 & 140 & 2821.9 & 56 & 196 \\
\hline 10 & Metal Decontamination Subsystem & 4 & 5 & 25 & 625 & 3571.6 & 250 & 875 \\
\hline 11 & Special Waste & 3 & 12 & 200 & & & & \\
\hline 12 & Mercury Amalgamation Subsystem & 1 & 2 & 10 & 255 & 1453.9 & 102 & 357 \\
\hline 13 & Lead Recovery Subsystem & 3 & 73 & 50 & 450 & 2571.7 & 180 & 630 \\
\hline 14 & Air Pollution Control Subsystem & 4 & 67 & 130 & 420 & 2400.8 & 168 & 588 \\
\hline 15 & Claytec Subsystem & 45 & 255 & 6,000 & 1,253 & 7158 & 501 & 1,754 \\
\hline 16 & Certification \& Shipping Subsystem & 175 & 12 & 70 & 840 & 8408.9 & 336 & 1,176 \\
\hline 17 & Support Subsystem & 5 & & & 480 & 4795.5 & 192 & 672 \\
\hline & Unit cost (\$/unit) & $\$ 140,000$ & & & & & & \\
\hline & Total Cost & 68,740 & 1964 & 10,082 & & & & 17,226 \\
\hline & Post Totals To Table 4-4, Item & 5.1 & 5.2 & 5.3 & & & & 5.4 \\
\hline
\end{tabular}

Notes:

1. Annual Maintenance Labor is $250 \%$ of maintenance material cost.

2. Maintenance Material is assumed to be $2 \%$ of equipment capital cost for the Bulk Soil Treatment subsystem, because the capital equipment is based on one shift operation.

3. Maintenance Material is assumed to be $10 \%$ of equipment capital cost for organic destruction and soft debris

4. Maintenance Material is assumed to be $4 \%$ of equipment capital cost for Administration, Receiving \& Inspection,

Grout Stabilization, Certification \& Shipping, and Support subsystems.

5. Maintenance Material is assumed to be $7 \%$ of equipment capital cost for all other subsystems 
Table B-4.5 : Decontamination \& Decommissioning Costs ENTS - 4 CWO, Desorption, Compaction and Polymer Stabilization

\begin{tabular}{|c|c|c|c|c|c|c|}
\hline & \multirow{3}{*}{ UNIT OPERATION } & \multicolumn{5}{|c|}{$\begin{array}{c}\text { ENTS - } 4 \\
\text { CWO, Desorption, Compaction and Polymer Stabilization }\end{array}$} \\
\hline & & \multicolumn{4}{|c|}{ Building Area } & \multirow[b]{2}{*}{$\begin{array}{c}\text { Total } \\
\text { Area Cost } \\
\$ 1000\end{array}$} \\
\hline & & $\begin{array}{c}\text { Cost } \\
\text { Category } 1 \\
\text { sq.ft }\end{array}$ & $\begin{array}{c}\text { Cost } \\
\text { Category } 2 \\
\text { sq.ft }\end{array}$ & \begin{tabular}{|c|}
$\begin{array}{c}\text { Cost } \\
\text { Category } 3 \\
\text { sq.ft }\end{array}$ \\
\end{tabular} & $\begin{array}{c}\text { Cost } \\
\text { Category } 4 \\
\text { sq.fit }\end{array}$ & \\
\hline & Administration Building Subsystem & 12,440 & 3,000 & & & $6, \overline{948}$ \\
\hline & Receiving \& Inspection Subsystem & & 24,227 & 2,970 & 14,839 & 18,916 \\
\hline 3 & Aqueous Waste Treatment Subsystem & & 1,150 & 1,751 & 1,590 & 2,021 \\
\hline 4 & Organic Destruction Subsystem & & 1,128 & 1,717 & 1,560 & 1,983 \\
\hline 5 & Residue \& Sludge Treatment Subsystem & & 2,040 & 3,105 & 2,820 & 3,584 \\
\hline 6 & Soft Debris Treatment Subsystem & & 1,606 & 2,444 & 2,220 & 2,822 \\
\hline \multicolumn{7}{|c|}{7 Complex Debris Treatment Subsystem } \\
\hline & Open Debris Treatment Subsystem & & 1,888 & 2,873 & 2,610 & 3,317 \\
\hline 9 & Bulk Soil Treatment Subsystem & & 1,606 & 2,444 & 2,220 & 2,822 \\
\hline 10 & Metal Decontamination Subsystem & & 641 & 169 & 1,904 & 1,222 \\
\hline 11 & Special Waste & & 604 & 159 & 1,792 & 1,150 \\
\hline 12 & Mercury Amalgamation Subsystem & & 1,020 & 1,552 & 1,410 & 1,792 \\
\hline 13 & Lead Recovery Subsystem & & & & & \\
\hline 14 & Air Pollution Control Subsystem & & 955 & 1,453 & 1,320 & 1,678 \\
\hline 15 & Claytec Subsystem & & 3,097 & 8,278 & 5,250 & 7,481 \\
\hline 16 & Certification \& Shipping Subsystem & & 28,277 & & & 12,725 \\
\hline \multirow[t]{3}{*}{17} & Support Subsystem & 3,360 & 16,883 & & & 9,109 \\
\hline & Total Cost & & 6 & & & 77,570 \\
\hline & & & Post Totals To & Table 4-4, Item & & 7.0 \\
\hline
\end{tabular}




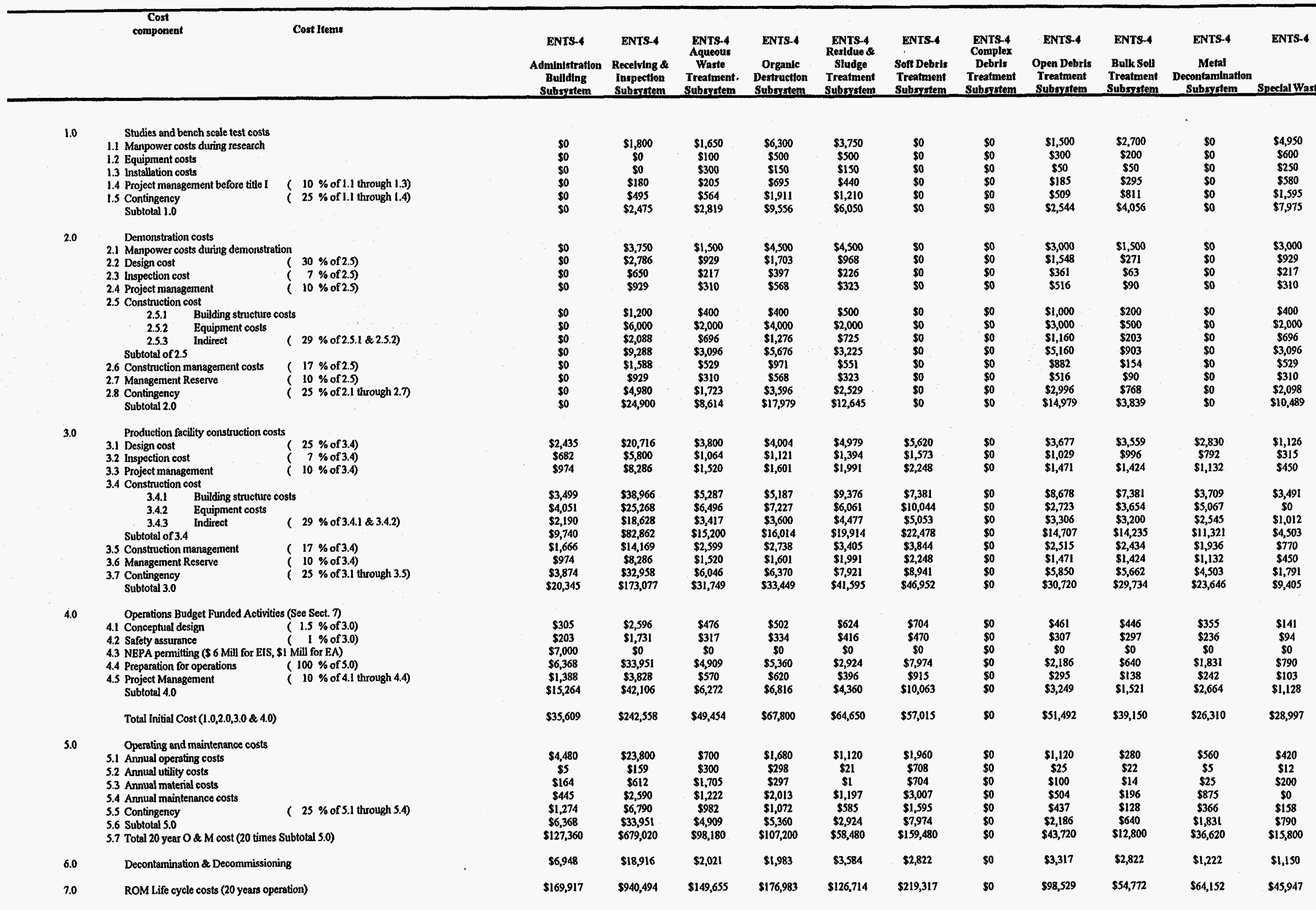


Table B- 4.6 :Total Life-Cycle Cost (without Disposal) Estimate Summary for

Enhanced Integrated Nonthermal Treatment Systems (cost module ENTS-4)

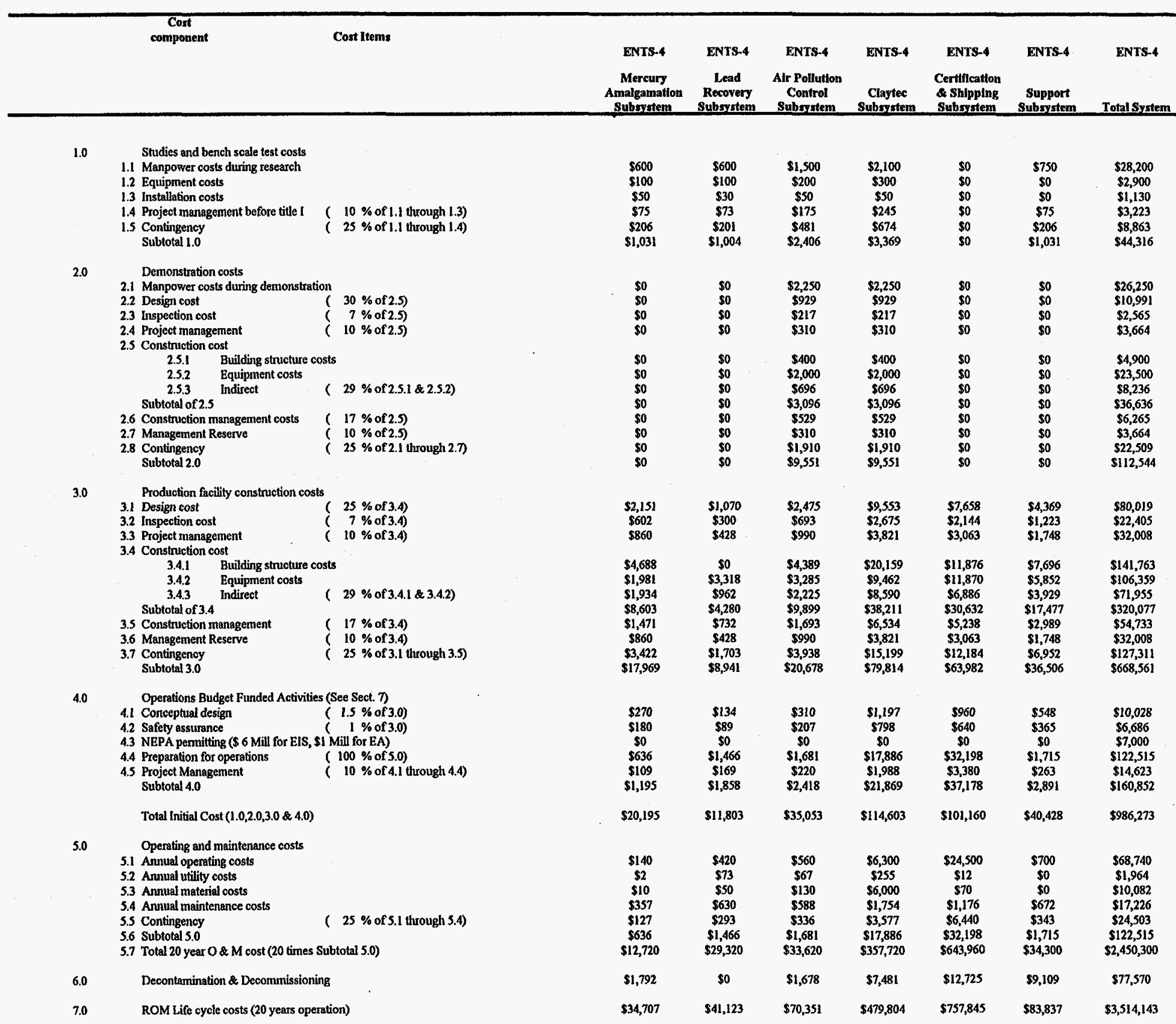




\section{B-5 ENTS-5 Cost Tables}

B-5.1. Equipment Purchase and Installation Budget Estimates

B-5.2. Development, Testing and Evaluation Cost Estimate

B-5.3. Building, Equipment and Installation Cost Summary

B-5.4. Annual Operating and Maintenance Costs

B-5.5. Decontamination and Decommissioning Costs

B-5.6 Total Life-Cycle Cost (without Disposal) Estimate Summary for Enhanced Integrated Nonthermal Treatment Systems 
Table B-5.1: Equipment Purchase and Installation Budget Estimates

Enhanced Non-Thermal Treatment System

ENTS - 5 CWO with Increased Reaction Rates

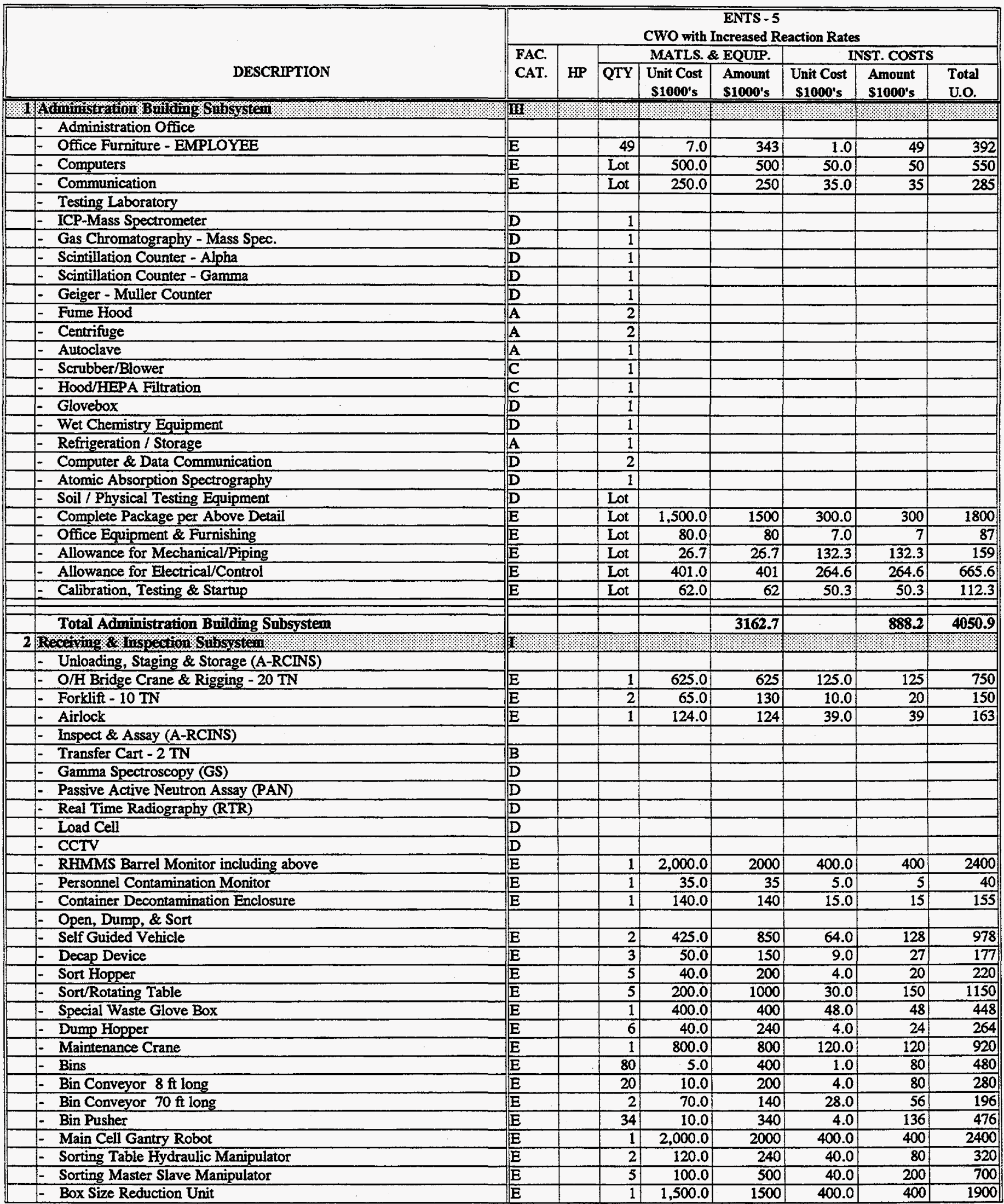


Table B-5.1: Equipment Purchase and Installation Budget Estimates Enhanced Non-Thermal Treatment System ENTS - 5 CWO with Increased Reaction Rates

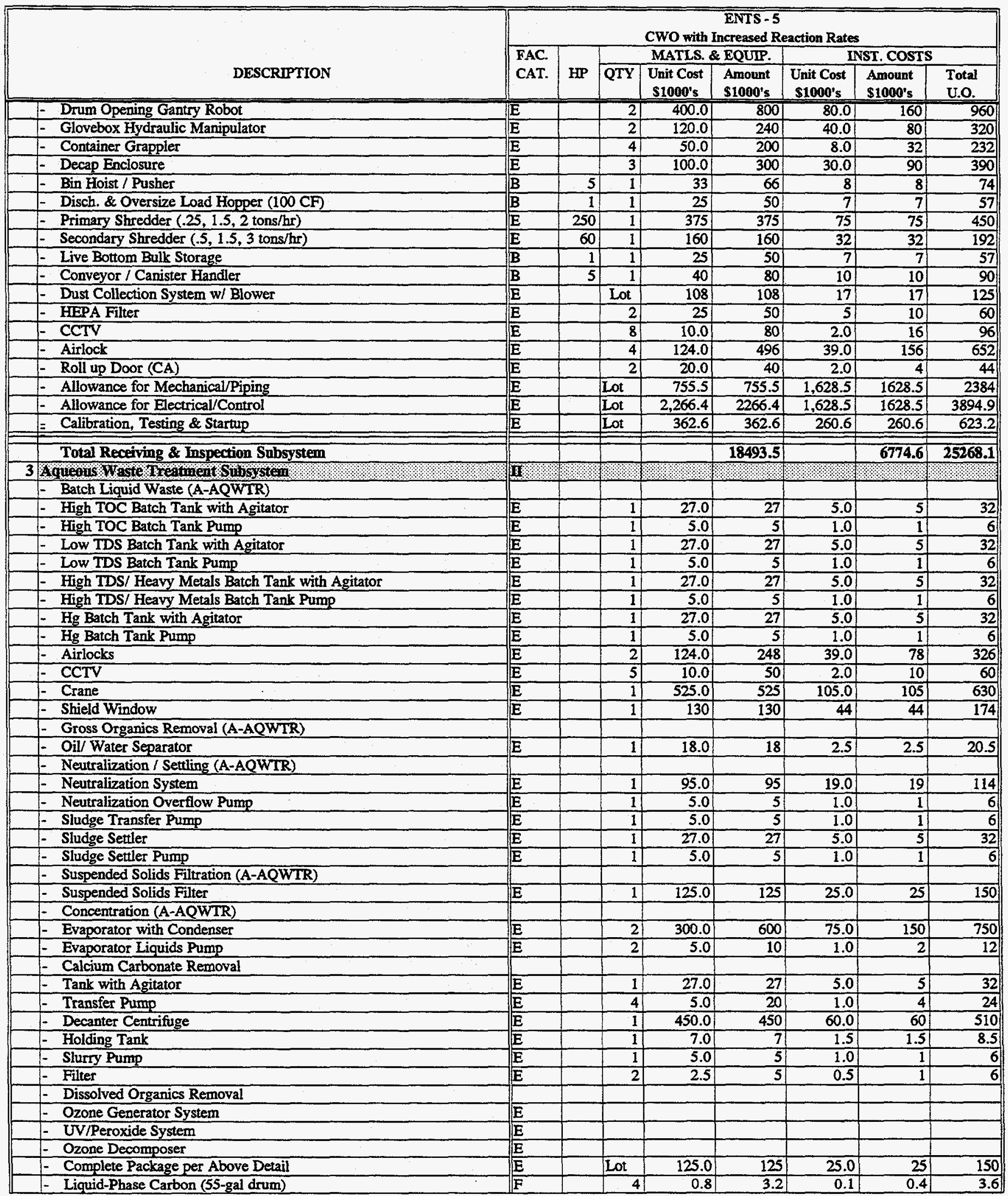


Table B-5.1: Equipment Purchase and Installation Budget Estimates

Enhanced Non-Thermal Treatment System

ENTS - 5 CWO with Increased Reaction Rates

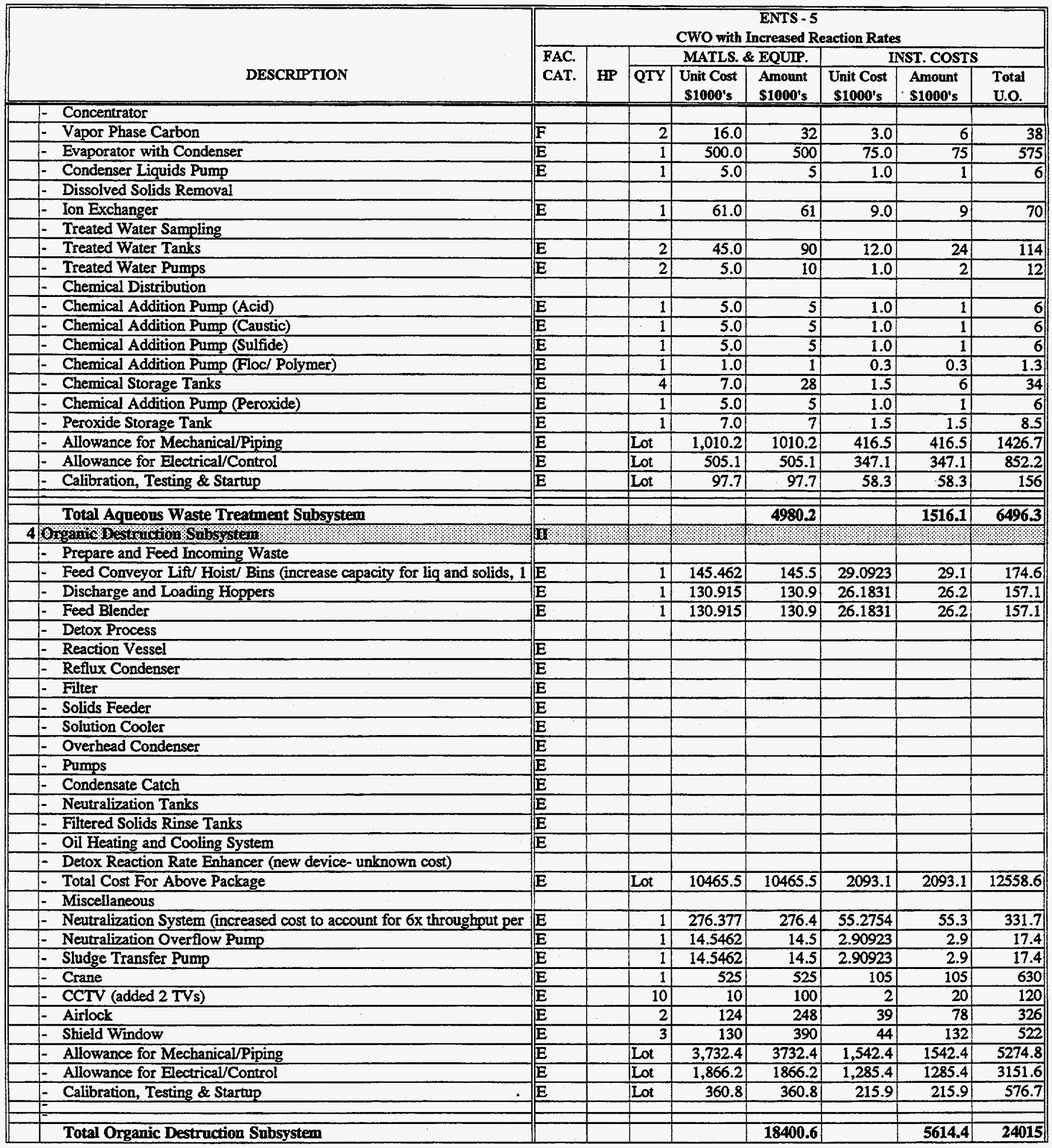


Table B-5.1: Equipment Purchase and Installation Budget Estimates

Enhanced Non-Thermal Treatment System

ENTS - 5 CWO with Increased Reaction Rates

\begin{tabular}{|c|c|c|c|c|c|c|c|c|}
\hline \multirow[b]{3}{*}{ DESCRIPTION } & \multicolumn{8}{|c|}{$\begin{array}{c}\text { ENTS - } 5 \\
\text { CWO with Increased Reaction Rates }\end{array}$} \\
\hline & \multirow{2}{*}{$\begin{array}{l}\text { FAC. } \\
\text { CAT. }\end{array}$} & \multirow[b]{2}{*}{ HP } & \multicolumn{3}{|c|}{ MATLS. \& EQUIP. } & \multicolumn{3}{|c|}{ INST. COSTS } \\
\hline & & & QTY & $\begin{array}{c}\text { Unit Cost } \\
\$ 1000 \text { 's }\end{array}$ & $\begin{array}{l}\text { Amount } \\
\$ 1000 \text { 's }\end{array}$ & $\begin{array}{c}\text { Unit Cost } \\
\$ 1000 ' s\end{array}$ & $\begin{array}{l}\text { Amount } \\
\text { S1000's }\end{array}$ & $\begin{array}{l}\text { Total } \\
\text { U.O. }\end{array}$ \\
\hline 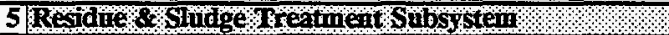 & T: & & & & & & \% & \\
\hline - Feed Preparation and Size Reduction & & & & & & & & \\
\hline - Feed Conveyor Lift/Hoist/Bins & E & & 1 & 50 & 50 & 8 & 8 & 58 \\
\hline - Discharge. \& Oversize Load Hopper (100 CF) & E & & 1 & 45 & 45 & 8 & 8 & 53 \\
\hline - Manipulate Crane 10 TN & E & 15 & 1 & 525 & 525 & 105 & 105 & 630 \\
\hline - Shield Window & $\mathbf{E}$ & & 1 & 130.0 & 130 & 44.0 & 44 & 174 \\
\hline - Airlock & E & & 2 & 124 & 248 & 39 & 78 & 326 \\
\hline - CCTV & $\mathbf{E}$ & & 10 & 10 & 100 & 2 & 20 & 120 \\
\hline - Feed Blender & $\mathbf{E}$ & & 1 & 45 & 45 & 8 & 8 & 53 \\
\hline - Dewatering System & E & & 1 & & & & & \\
\hline - THERMAL DESORPTION & & & & & & & & \\
\hline - High Vacuum Dryer & & & 1 & & & & & \\
\hline - Nitrogen gas storage and feed tank & $\mathbf{E}$ & & 1 & & & & & \\
\hline - Vacuum Pump & E & & 1 & & & & & \\
\hline - Filter & $\mathbf{E}$ & & 1 & & & & & \\
\hline - Hot Oil System & & & & & & & & \\
\hline - Oil Storage and Feed Tank & E & & 1 & & & & & \\
\hline - Oil Heater & E & & 1 & & & & & \\
\hline - Fuel Storage and Feed Tank & $E$ & & 1 & & & & & \\
\hline - Condensers & & & & & & & & \\
\hline - HEPA Filter for particulates & E & & $1]$ & & & & & \\
\hline - Induced Air Fan & $\mathbf{E}$ & & 1 & & & & & \\
\hline - Primary Condensers (Air Cooler) & $\mathbf{E}$ & & 1 & & & & & \\
\hline - Secondary Condensers (Water Chiller) & $\mathbf{E}$ & & 2 & & & & & \\
\hline - Total per Package Above & E & & Lot & 1700 & 1700 & 170 & 170 & 1870 \\
\hline - Mercury Removal System & & & & & & & & \\
\hline - Mercury Precipitation Tank & E & & 1 & 95 & 95. & $19]$ & 19 & 114 \\
\hline - Iodine Recovery Tank & E & & 1 & 27 & 27. & 5 & 5 & 32 \\
\hline - KI/2 Storage and Feed & E & & 1 & 27. & 27. & 5 & 5 & 32 \\
\hline - Metal Precipitation Tank & E & & 1 & 27 & 27 & 5 & 5 & 32 \\
\hline - Mercury Reduction Tank & $\mathbf{E}$ & & 1 & 27 & 27 & 5 & 5 & 32 \\
\hline - Solids Wash Tank & $\mathbf{E}$ & & 1 & 27 [ & 27 & 5 & 5 & 32 \\
\hline - Water Holding Tank & E & & 1 & 12 & 12 & 3 & 3 & 15 \\
\hline - Acid Holding and Feed Tank & E & & 1 & 12 & 12 & 3 & 3 & 15 \\
\hline - $\mathrm{H}_{2} \mathrm{O}_{2}$ Holding and Feed Tank & $\mathbf{E}$ & & 1 & 12 & 12 & 3 & 3 & 15 \\
\hline - Lime Holding Tank & $\mathbf{E}$ & & $1]$ & 12 & 12 & 3 & 3 & 15 \\
\hline - Rinse Tank & E & & $1]$ & 12 & 12 & 3 & 3 & 15 \\
\hline - Filter & $\mathbf{E}$ & & 1 & 125 & 125 & 25 & 25 & 150 \\
\hline - Pump & $\mathbf{E}$ & & 10 & 5 & 50 & 1 & 10. & 60 \\
\hline - Allowance for Mechanical/Control/Instrumentation & $\mathbf{E}$ & & Lot & 992.4 & 992.4 & 321.0 & 321 & 1313.4 \\
\hline - Allowance For Electrical/Control & E & & Lot & 496.2 & 496.2 & 267.5 & 267.5 & 763.7 \\
\hline - Calibration, Testing \& Startup & $E$ & & Lot & 95.9 & 95.9 & 44.9 & 44.9 & 140.8 \\
\hline Total Residue \& Slndge Treatment Subsystem & & & & & 4892.5 & & 1168.4 & 6060.9 \\
\hline 6 Softoblob T Teatment Subsy Sten & H & & & & ? & & & \\
\hline - Prepare \& Feed Incoming Waste & & & & & & & & \\
\hline - Feed Conveyor Lift/ Hoist/Bins & $\mathbf{E}$ & & $1)$ & & & & & \\
\hline - Discharge and Loading Hoppers & $\mathbf{E}$ & & 1) & & & & & \\
\hline - Feed Blender & E & & $1)$ & & & & & \\
\hline - Detox Process & 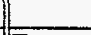 & & & & & & & \\
\hline - Reaction Vessel & E & & & & & & & \\
\hline - Reflux Condenser & E & & & & & & & \\
\hline - Filter & E & & & & & & & \\
\hline - Solids Feeder & E & & & & & & & \\
\hline - Solution Cooler & E & & & & & & & \\
\hline - Overhead Condenser & $E$ & & & & & & & \\
\hline - Pumps & E & & & & & & & \\
\hline - Condensate Catch & E & & & & & & & \\
\hline - Neutralization Tanks & $\|$ E & & & & & & & \\
\hline
\end{tabular}


Table B-5.1: Equipment Purchase and Installation Budget Estimates Enhanced Non-Thermal Treatment System ENTS - 5 CWO with Increased Reaction Rates

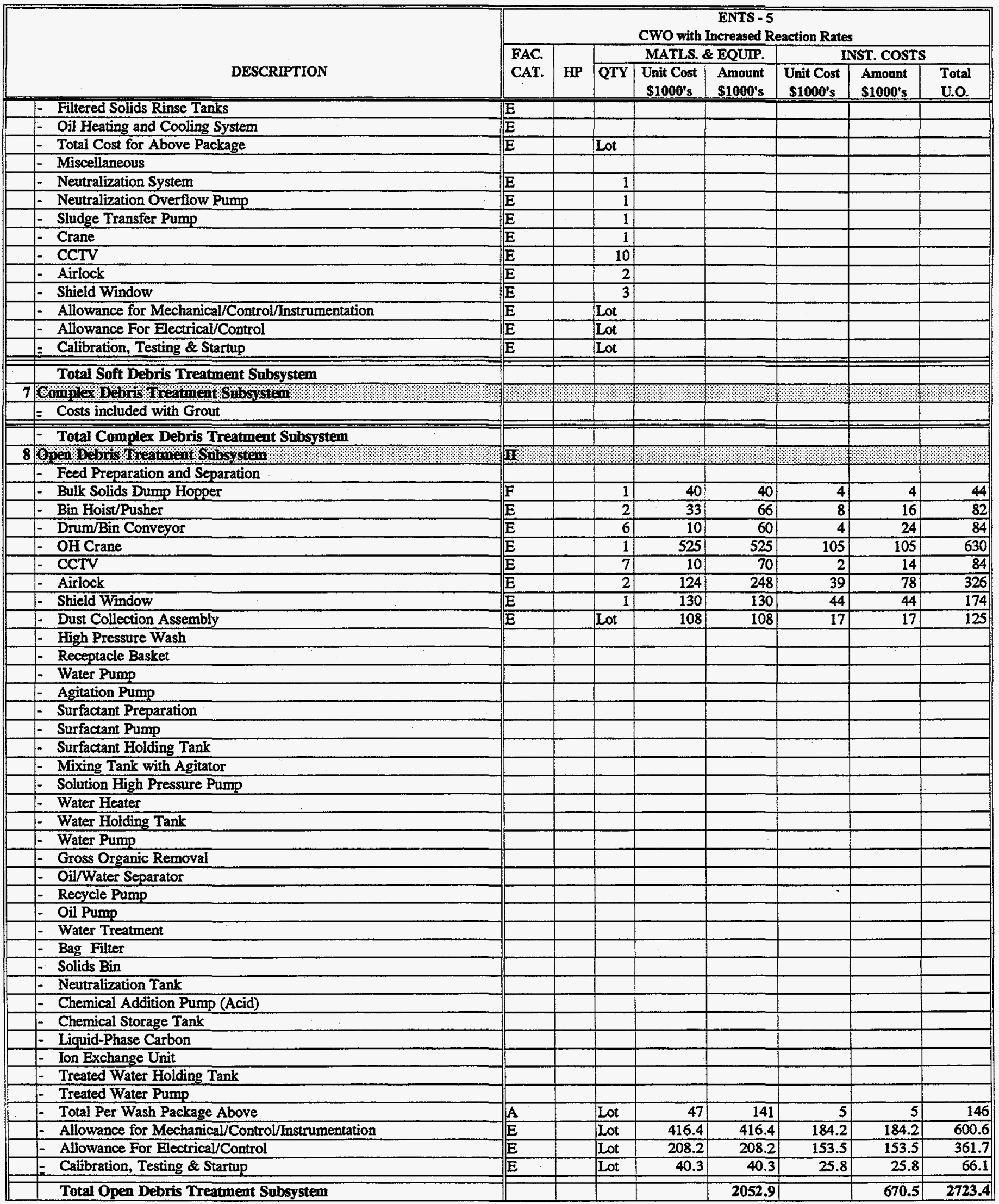


Table B-5.1: Equipment Purchase and Installation Budget Estimates Enhanced Non-Thermal Treatment System ENTS - 5 CWO with Increased Reaction Rates

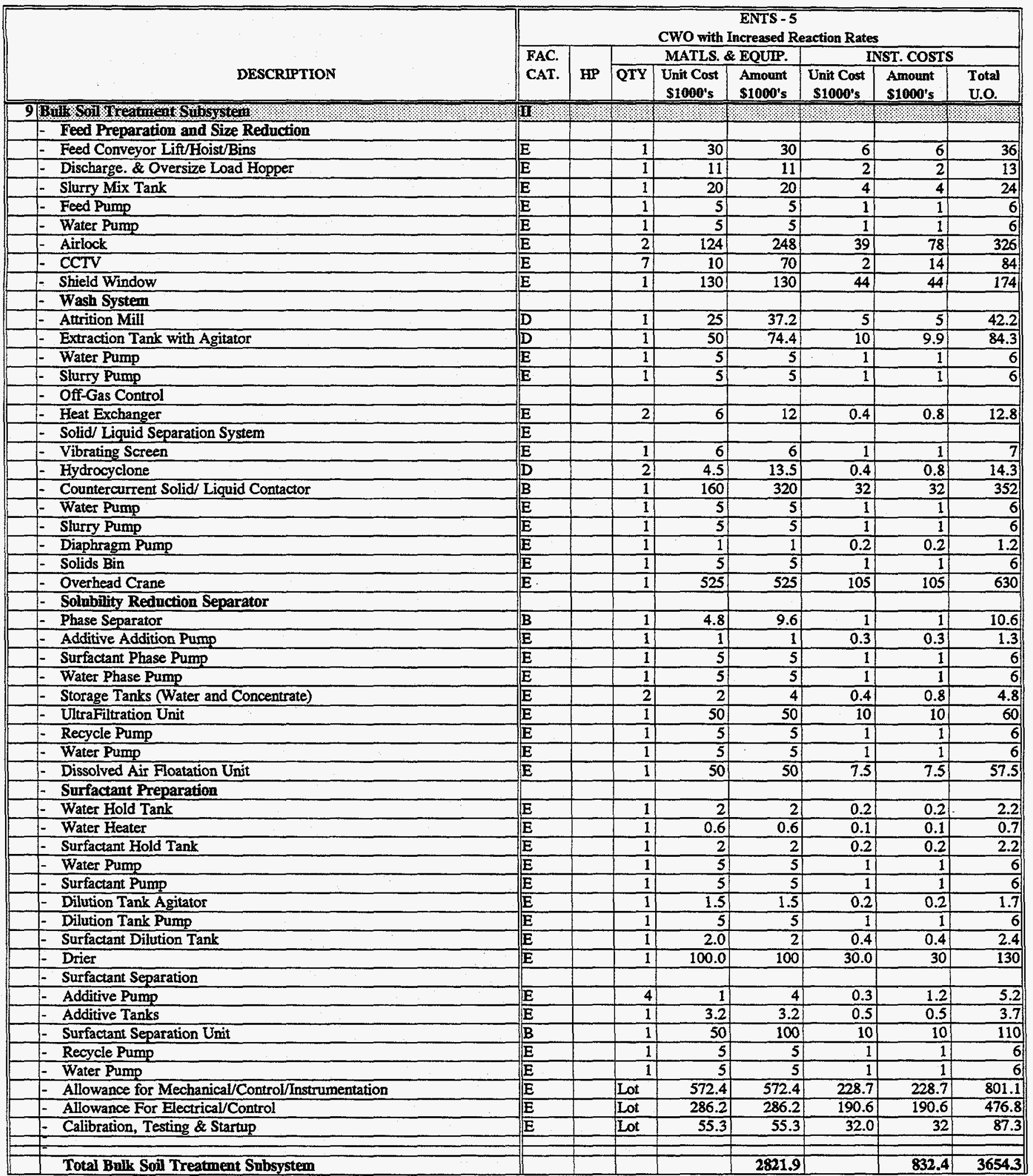


Table B-5.1: Equipment Purchase and Installation Budget Estimates

Enhanced Non-Thermal Treatment System

ENTS - 5 CWO with Increased Reaction Rates

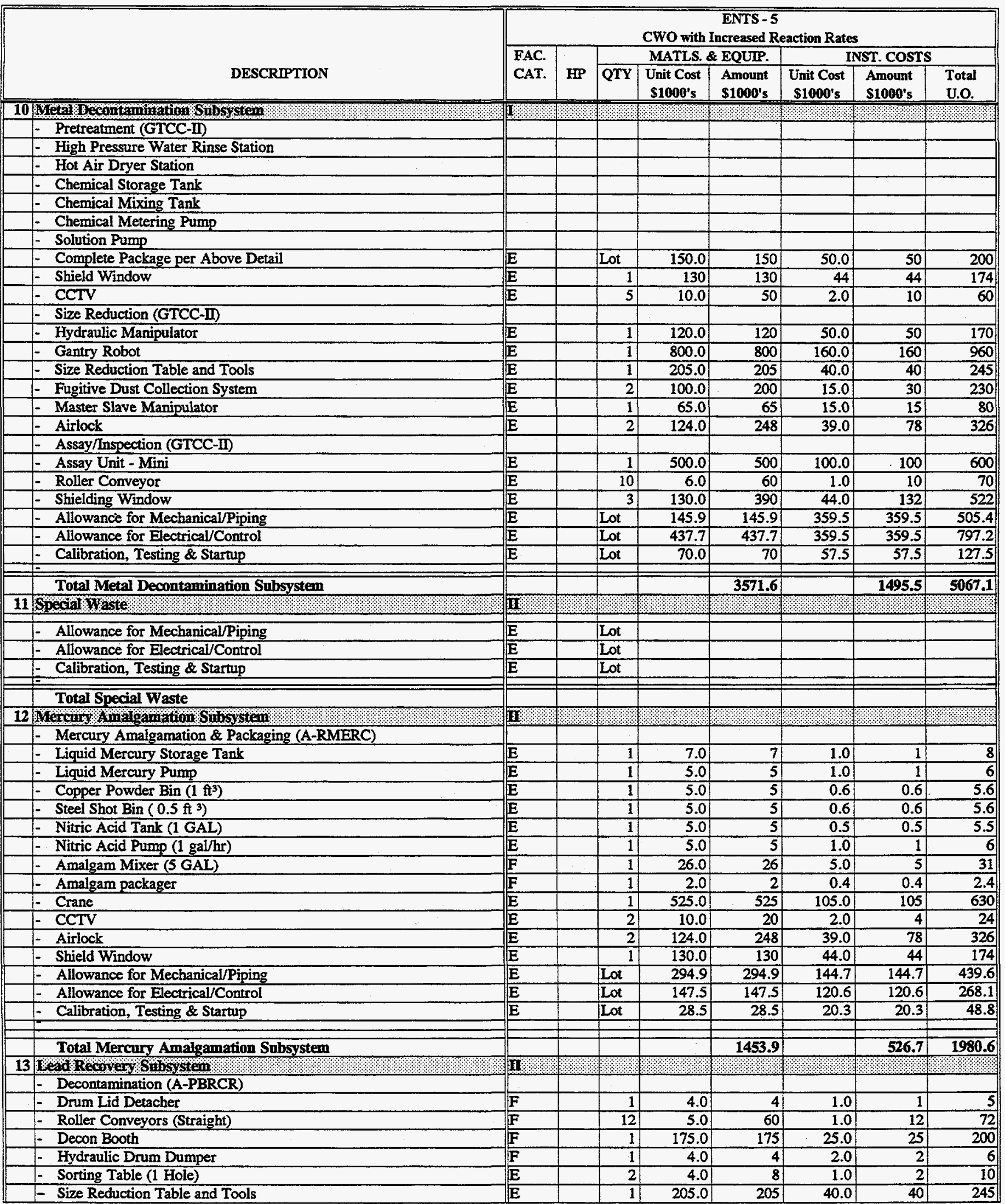


Table B-5.1: Equipment Purchase and Installation Budget Estimates Enhanced Non-Thermal Treatment System ENTS - 5 CWO with Increased Reaction Rates

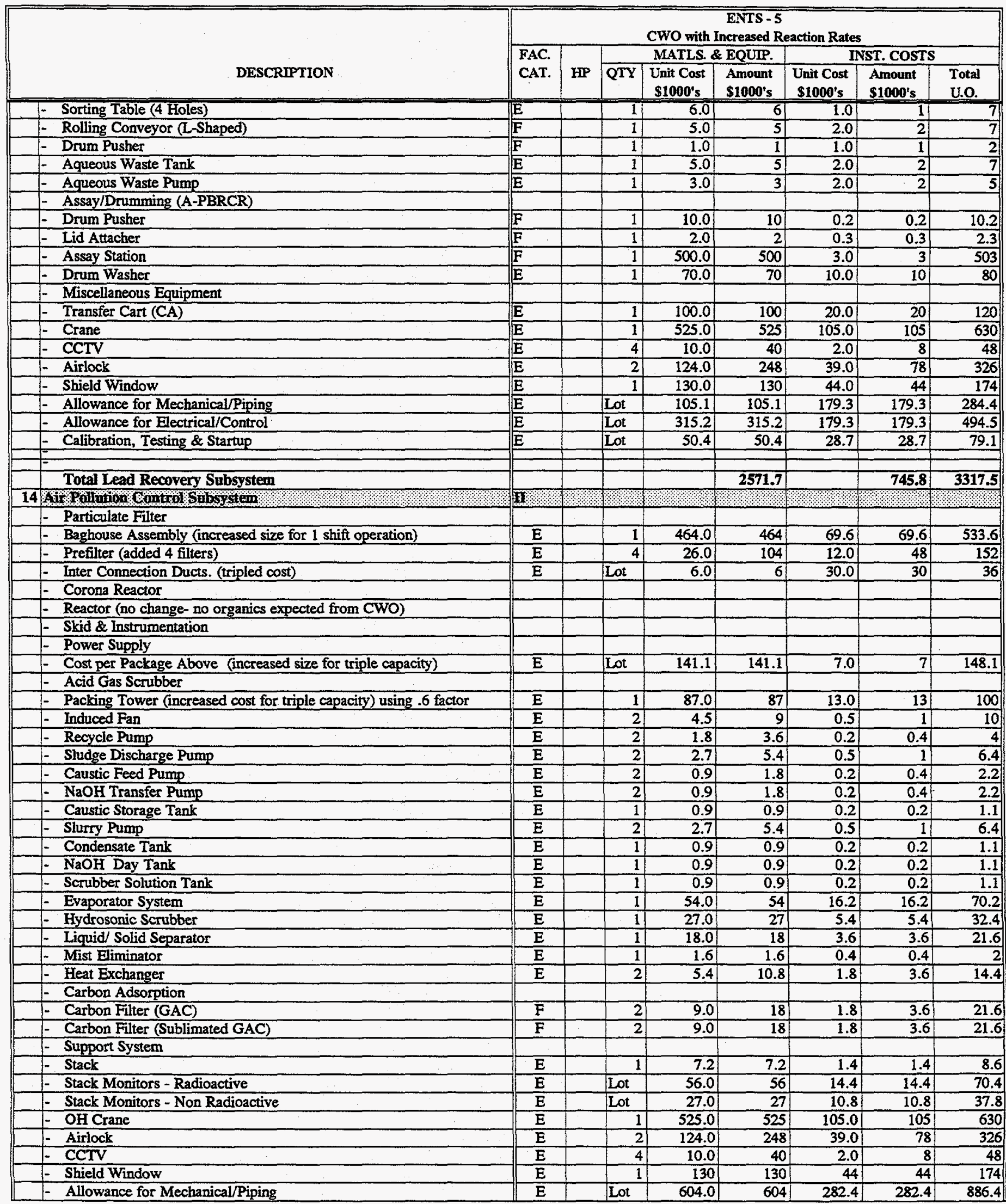


Table B-5.1: Equipment Purchase and Installation Budget Estimates

Enhanced Non-Thermal Treatment System

ENTS - 5 CWO with Increased Reaction Rates

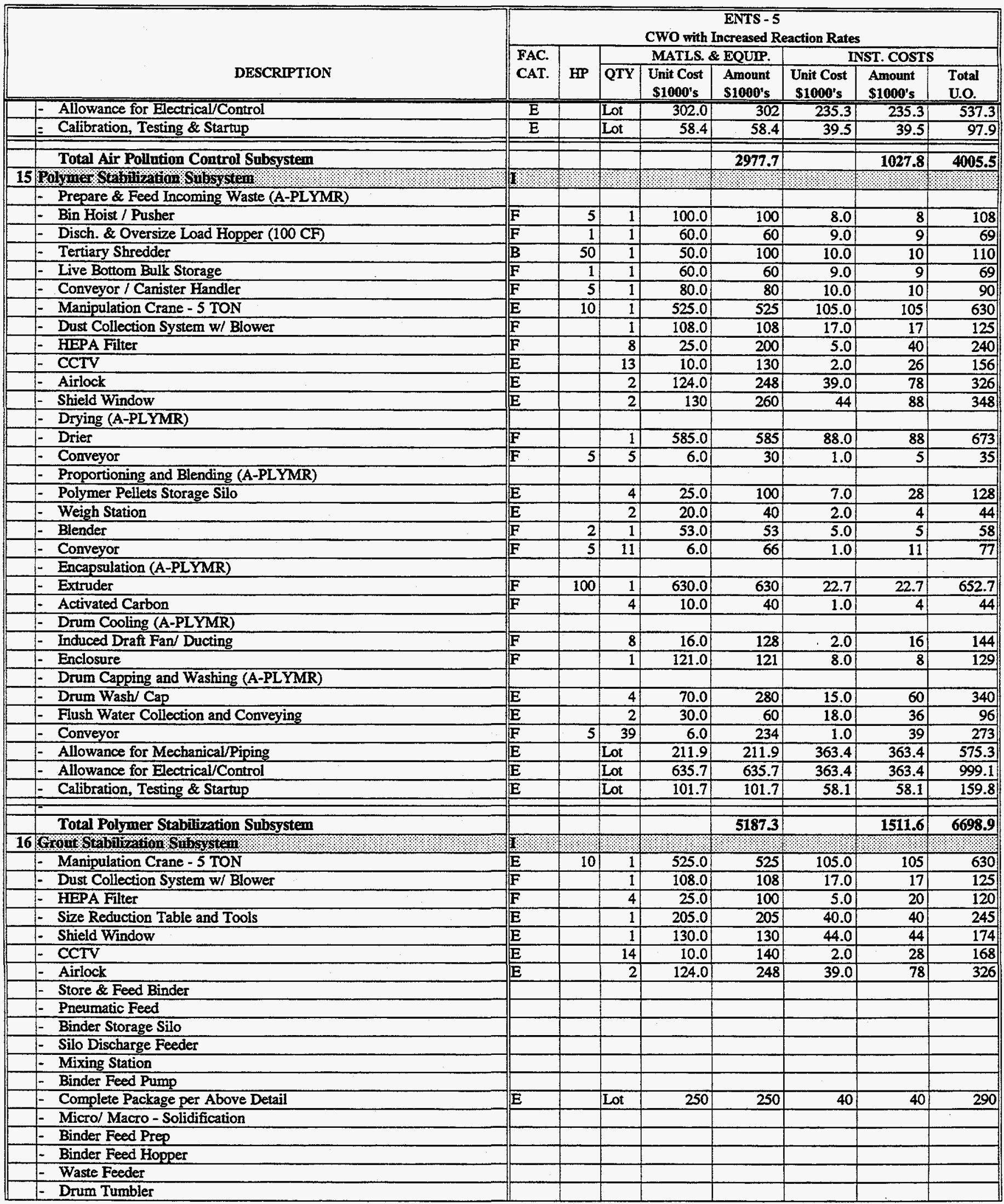


Table B-5.1: Equipment Purchase and Installation Budget Estimates Enhanced Non-Thermal Treatment System ENTS - 5 CWO with Increased Reaction Rates

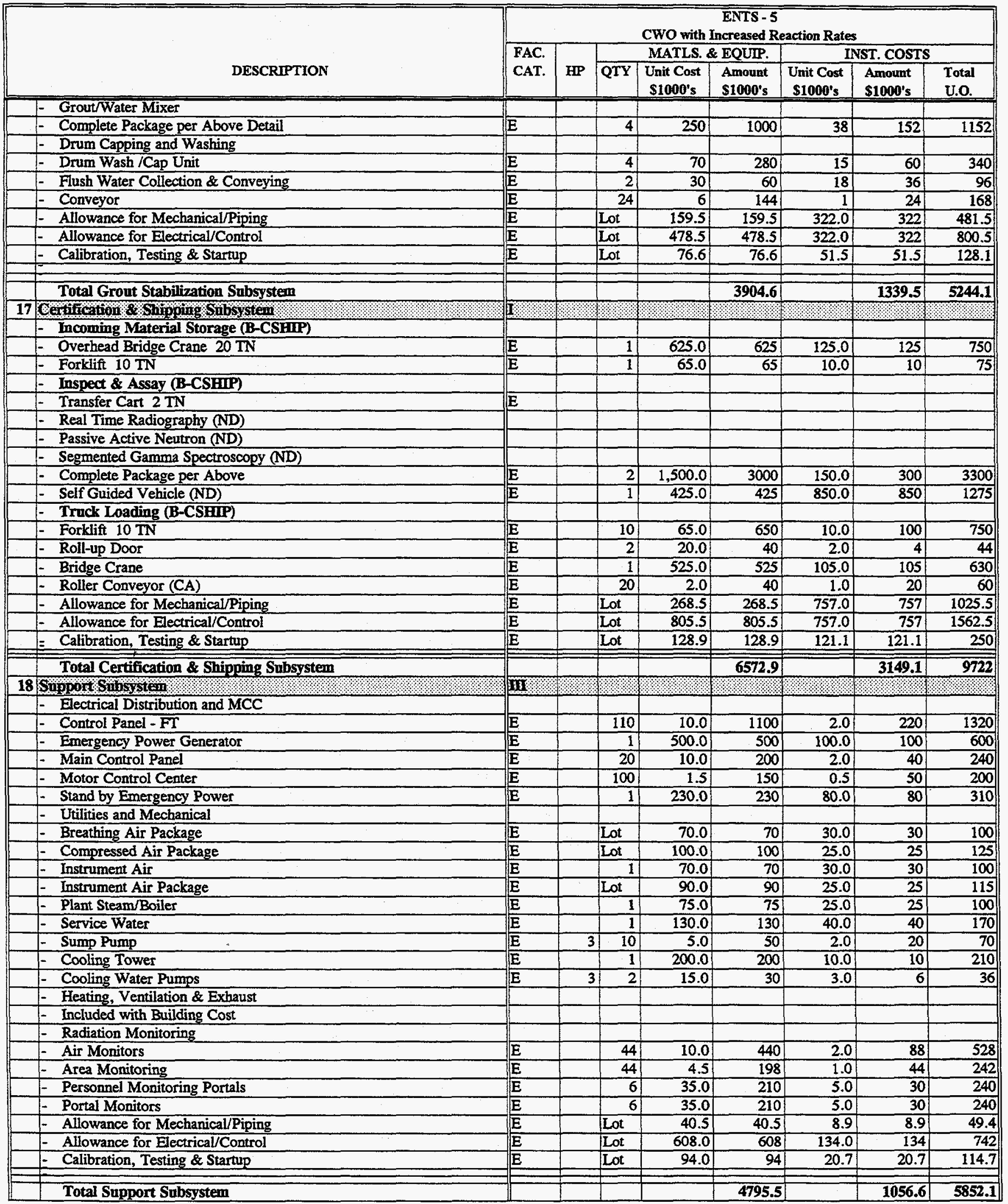


Table B-5.2 : Development, Testing \& Evaluation Cost Estimate ENTS - 5 CWO with Increased Reaction Rates

\begin{tabular}{|c|c|c|c|c|c|c|c|c|c|c|}
\hline \multirow{2}{*}{ UNIT OPERATION } & \multirow{2}{*}{ UNIT OPERATION } & \multicolumn{9}{|c|}{$\begin{array}{c}\text { ENTS-5 } \\
\text { CWO with Increased Reaction Rates }\end{array}$} \\
\hline & & $\begin{array}{c}\text { Primary } \\
\text { Paper }\end{array}$ & $\begin{array}{l}\text { Bench } \\
\text { Scale }\end{array}$ & $\begin{array}{c}\text { Sec. } \\
\text { Paper }\end{array}$ & & $\begin{array}{l}\text { Bench } \\
\text { Scale }\end{array}$ & $\begin{array}{l}\text { Bench } \\
\text { Scale }\end{array}$ & $\begin{array}{c}\text { Pilot } \\
\text { Plant }\end{array}$ & $\begin{array}{l}\text { Pilot } \\
\text { Plant }\end{array}$ & $\begin{array}{l}\text { Pilot } \\
\text { Plant }\end{array}$ \\
\hline \multicolumn{11}{|c|}{1 Administration Building Subsystem } \\
\hline 2 & Receiving \& Inspection Subsystem & 12 & & & 12 & & & 25 & 1200 & 6000 \\
\hline 3 & Aqueous Waste Treatment Subsystem & 5 & 3 & 3 & 11 & 100 & 300 & 10 & 400 & 2,000 \\
\hline 4 & Organic Destruction Subsystem & 18 & 20 & 4 & 42 & 500 & 150 & 30 & 400 & 4,000 \\
\hline 5 & Residue \& Sludge Treatment Subsystem & 13 & 8 & 4 & 25 & 500 & 150 & 30 & 500 & 2,000 \\
\hline \multicolumn{11}{|c|}{6 Soft Debris Treatment Subsystem } \\
\hline \multicolumn{11}{|c|}{7 Complex Debris Treatment Subsystem } \\
\hline 8 & Open Debris Treatment Subsystem & 2 & 6 & 2 & 10 & 300 & 50 & 20 & 1,000 & 3,000 \\
\hline 9 & Bulk Soil Treatment Subsystem & 10 & 6 & 2 & 18 & 200 & 50 & 10 & 200 & 500 \\
\hline 10 & Metal Decontamination Subsystem & & & & & & & & & \\
\hline 11 & Special Waste & 8 & 20 & 5 & 33 & 600 & 250 & 20 & 400 & 2,000 \\
\hline 12 & Mercury Amalgamation Subsystem & 1 & 3 & & 4 & 100 & 50 & & & \\
\hline 13 & Lead Recovery Subsystem & 2 & 2 & & 4 & 100 & 30 & & & \\
\hline 14 & Air Pollution Control Subsystem & 5 & 3 & 2 & 10 & 200 & 50 & 15 & 400 & 2,000 \\
\hline 15 & Polymer Stabilization Subsystem & 2 & 8 & 4 & 14 & 300 & 50 & 15 & 400 & 2,000 \\
\hline 16 & Grout Stabilization Subsystem & 2 & 8 & 4 & 14 & 300 & 50 & 15 & 400 & 1,000 \\
\hline 17 & Certification \& Shipping Subsystem & & & & & & & & & \\
\hline \multirow{4}{*}{18} & Support Subsystem & 5 & & & 5 & & & & & \\
\hline & Unit Cost (\$/Unit) & & & & $\$ 150,000$ & & & $\$ 150,000$ & & \\
\hline & Total Cost & & & & 30,300 & 3,200 & 1,180 & 28,500 & 5,300 & 24,500 \\
\hline & \multicolumn{4}{|c|}{ Post Totals To Table 4-5, Item } & 1.1 & 1.2 & 1.3 & 2.1 & 2.5 .1 & 2.5 .2 \\
\hline
\end{tabular}


Table B-5.3 : Building, Equipment and Installation Cost Summary ENTS - 5 CWO with Increased Reaction Rates

\begin{tabular}{|c|c|c|c|c|c|c|c|c|c|c|}
\hline & \multirow[b]{3}{*}{ UNIT OPERATION } & \multicolumn{9}{|c|}{$\begin{array}{c}\text { ENTS-5 } \\
\text { CWO with Increased Reaction Rates }\end{array}$} \\
\hline & & \multicolumn{5}{|c|}{ Building Area } & \multicolumn{3}{|c|}{ Material \& Equipment Costs } & \multirow{2}{*}{$\begin{array}{c}\text { Total } \\
\text { Cost per } \\
\text { Unit Oprtn } \\
\$ 1000 \\
\end{array}$} \\
\hline & & $\begin{array}{c}\text { Cost } \\
\text { Category } 1 \\
\text { sq.ft } \\
\end{array}$ & $\begin{array}{c}\text { Cost } \\
\text { Category } 2 \\
\text { sq.ft } \\
\end{array}$ & $\begin{array}{c}\text { Cost } \\
\text { Category } 3 \\
\text { sq.ft } \\
\end{array}$ & $\begin{array}{c}\text { Cost } \\
\text { Category } 4 \\
\text { sq.ft } \\
\end{array}$ & $\begin{array}{c}\text { Total } \\
\text { Area Cost } \\
\$ 1000 \\
\end{array}$ & \begin{tabular}{|c|}
$\begin{array}{c}\text { Purchase } \\
\text { Cost } \\
\$ 1000 \\
\end{array}$ \\
\end{tabular} & $\begin{array}{c}\text { Installation } \\
\text { Cost } \\
\$ 1000 \\
\end{array}$ & $\begin{array}{c}\text { Total } \\
\text { Cost } \\
\$ 1000 \\
\end{array}$ & \\
\hline 1.0 & Administration Building Subsystem & 12,440 & 3,000 & & & 3,499 & 3,163 & $8 \overline{88}$ & 4,051 & 7,550 \\
\hline 2.0 & Receiving \& Inspection Subsystem & & 24,227 & 2,970 & 14,839 & 38,966 & 18,494 & 6,775 & 25,268 & 64,234 \\
\hline 3.0 & Aqueous Waste Treatment Subsystem & & 1,150 & 1,751 & 1,590 & 5,287 & 4,980 & 1,516 & 6,496 & 11,783 \\
\hline 4.0 & Organic Destruction Subsystem & & 1,578 & 2,317 & 2,460 & 7,625 & 18,401 & 5,614 & 24,015 & 31,640 \\
\hline 5.0 & Residue \& Sludge Treatment Subsystem & & 2,040 & 3,105 & 2,820 & 9,377 & 4,893 & 1,168 & 6,061 & 15,438 \\
\hline 6.0 & Soft Debris Treatment Subsystem & & & & & & & & & \\
\hline 7.0 & Complex Debris Treatment Subsystem & & & & & & & & & \\
\hline 8.0 & Open Debris Treatment Subsystem & & 1,888 & 2,873 & 2,610 & 8,678 & 2,053 & 671 & 2,723 & 11,401 \\
\hline 9.0 & Bulk Soil Treatment Subsystem & & 1,606 & 2,444 & 2,220 & 7,381 & 2,822 & 832 & 3,654 & 11,035 \\
\hline 10.0 & Metal Decontamination Subsystem & & 641 & 169 & 1904 & 3,709 & 3,572 & 1,496 & 5,067 & 8,776 \\
\hline 11.0 & Special Waste & & 604 & 159 & 1792 & 3,491 & & & & 3,491 \\
\hline 12.0 & Mercury Amalgamation Subsystem & & 1020 & 1552 & 1410 & 4,688 & 1,454 & 527 & 1,981 & 6,669 \\
\hline 13.0 & Lead Recovery Subsystem & & & & & & 2,572 & 746 & 3,318 & 3,318 \\
\hline 14.0 & Air Pollution Control Subsystem & & 955 & 1,453 & 1,320 & 4,389 & 2,978 & 1,028 & 4,006 & 8,395 \\
\hline 15.0 & Polymer Stabilization Subsystem & & 2,322 & 3,534 & 3,210 & 10,673 & 5,187 & 1,512 & 6,699 & 17,372 \\
\hline 16.0 & Grout Stabilization Subsystem & & 1,931 & 2,940 & 2,670 & 8,878 & 3,905 & 1,340 & 5,244 & 14,122 \\
\hline 17.0 & Certification \& Shipping Subsystem & & 28,277 & & & 11,876 & 6,573 & 3,149 & 9,722 & 21,598 \\
\hline 18.0 & Support Subsystem & 3,360 & 16,883 & & & 7,696 & 4,796 & 1,057 & 5,852 & 13,548 \\
\hline & Total Cost & & & & & 136,213 & & & 114,157 & 250,370 \\
\hline & & & Post Totals To & Table 4-5, Ite & & 3.4 .1 & & & 3.4 .2 & \\
\hline
\end{tabular}


Table B-5.4 : Annual Operating \& Maintenance Costs ENTS - 5 CWO with Increased Reaction Rates

\begin{tabular}{|c|c|c|c|c|c|c|c|c|}
\hline & \multirow[b]{2}{*}{ UNIT OPERATION } & \multicolumn{7}{|c|}{$\begin{array}{c}\text { ENTS-5 } \\
\text { CWO with Increased Reaction Rates }\end{array}$} \\
\hline & & $\begin{array}{l}\text { Operating } \\
\text { FTE }\end{array}$ & $\begin{array}{l}\text { Utilities } \\
\$ 1000\end{array}$ & $\begin{array}{c}\text { Materials } \\
\$ 1000\end{array}$ & $\begin{array}{c}\text { Maintenance } \\
\text { Labor (1) } \\
\$ 1000\end{array}$ & $\$ 1000$ & $\begin{array}{c}\text { Maintenance } \\
\text { Materials (2) } \\
\$ 1000\end{array}$ & $\begin{array}{l}\text { Totals } \\
\$ 1000\end{array}$ \\
\hline 1 & Administration Building Subsystem & 32 & 5 & 164 & 318 & 3162.7 & 127 & 445 \\
\hline 2 & Receiving \& Inspection Subsystem & 170 & 159 & 612 & 1,850 & 18493.5 & 740 & 2,590 \\
\hline 3 & Aqueous Waste Treatment Subsystem & 5 & 300 & 1,705 & 873 & 4980.2 & 349 & 1,222 \\
\hline 4 & Organic Destruction Subsystem & 15 & 298 & 297 & 4,600 & 18400.6 & 1,840 & 6,440 \\
\hline 5 & Residue \& Sludge Treatment Subsystem & 8 & 21 & 1 & 855 & 4892.5 & 342 & 1,197 \\
\hline 6 & Soft Debris Treatment Subsystem & & & & & & & \\
\hline 7 & Complex Debris Treatment Subsystem & & & & & & & \\
\hline 8 & Open Debris Treatment Subsystem & 8 & 25 & 100 & 360 & 2052.9 & 144 & 504 \\
\hline 9 & Bulk Soil Treatment Subsystem & 2 & 22 & 14 & 140 & 2821.9 & 56 & 196 \\
\hline 10 & Metal Decontamination Subsystem & 4 & 5 & 25 & 625 & 3571.6 & 250 & 875 \\
\hline 11 & Special Waste & 3 & 12 & 200 & & & & \\
\hline 12 & Mercury Amalgamation Subsystem & 1 & 2 & 10 & 255 & 1453.9 & 102 & 357 \\
\hline 13 & Lead Recovery Subsystem & 3 & 73 & 50 & 450 & 2571.7 & 180 & 630 \\
\hline 14 & Air Pollution Control Subsystem & 4 & 67 & 130 & 520 & 2977.7 & 208 & 728 \\
\hline 15 & Polymer Stabilization Subsystem & 37.5 & 118 & 1,858 & 908 & 5187.3 & 363 & 1,271 \\
\hline 16 & Grout Stabilization Subsystem & 8 & 47 & 847 & 390 & 3904.6 & 156 & 546 \\
\hline 17 & Certification \& Shipping Subsystem & 88 & 12 & 70 & 658 & 6572.9 & 263 & 921 \\
\hline 18 & Support Subsystem & 5 & & & 480 & 4795.5 & 192 & 672 \\
\hline & Unit cost (\$/unit) & $\$ 140,000$ & & & & & & \\
\hline & Total Cost & 55,090 & 1166 & 6,083 & & & & 18,594 \\
\hline & Post Totals To Table 4-5, Item & 5.1 & 5.2 & 5.3 & & & & 5.4 \\
\hline
\end{tabular}

Notes:

1. Annual Maintenance Labor is $250 \%$ of maintenance material cost.

2. Maintenance Material is assumed to be $2 \%$ of equipment capital cost for the Bulk Soil Treatment subsystem, because the capital equipment is based on one shift operation.

3. Maintenance Material is assumed to be $10 \%$ of equipment capital cost for organic destruction and soft debris.

4. Maintenance Material is assumed to be $4 \%$ of equipment capital cost for Administration, Receiving \& Inspection,

Grout Stabilization, Certification \& Shipping, and Support subsystems.

5. Maintenance Material is assumed to be $7 \%$ of equipment capital cost for all other subsystems

6. Organic destruction FTEs include one shift operation of CWO process (3 FTEs), but allow 3 shift FTEs for material handling of soft debris (4 FTE 
Table B-5.5 : Decontamination \& Decommissioning Costs ENTS - 5 CWO with Increased Reaction Rates

\begin{tabular}{|c|c|c|c|c|c|c|}
\hline & \multirow{4}{*}{ UNIT OPERATION } & \multicolumn{5}{|c|}{$\begin{array}{c}\text { ENTS-5 } \\
\text { CWO with Increased Reaction Rates }\end{array}$} \\
\hline & & \multicolumn{4}{|c|}{ Building Area } & \multirow[b]{2}{*}{$\begin{array}{c}\text { Total } \\
\text { Area Cost }\end{array}$} \\
\hline & & \begin{tabular}{|l} 
Cost \\
Category 1
\end{tabular} & \begin{tabular}{|c|} 
Cost \\
Category 2
\end{tabular} & \begin{tabular}{|c|} 
Cost \\
Category 3
\end{tabular} & \begin{tabular}{|c|} 
Cost \\
Category 4
\end{tabular} & \\
\hline & & \begin{tabular}{|l|} 
sq.ft \\
\end{tabular} & sq.ft & sq.ft & sq.ft & $\$ 1000$ \\
\hline & Administration Building Subsystem & 12,440 & 3,000 & & & $6, \overline{948}$ \\
\hline & Receiving \& Inspection Subsystem & & 24,227 & 2,970 & 14,839 & 18,916 \\
\hline & Aqueous Waste Treatment Subsystem & & 1,150 & 1,751 & 1,590 & 2,021 \\
\hline & Organic Destruction Subsystem & & 1,578 & 2,317 & 2,460 & 2,860 \\
\hline & Residue \& Sludge Treatment Subsystem & & 2,040 & 3,105 & 2,820 & 3,584 \\
\hline \multicolumn{7}{|c|}{6 Soft Debris Treatment Subsystem } \\
\hline \multicolumn{7}{|c|}{7 Complex Debris Treatment Subsystem } \\
\hline & Open Debris Treatment Subsystem & & 1,888 & 2,873 & 2,610 & 3,317 \\
\hline & Bulk Soil Treatment Subsystem & & 1,606 & 2,444 & 2,220 & 2,822 \\
\hline & Metal Decontamination Subsystem & & 641 & 169 & 1,904 & 1,222 \\
\hline & Special Waste & & 604 & 159 & 1,792 & 1,150 \\
\hline & Mercury Amalgamation Subsystem & & 1,020 & 1,552 & 1,410 & 1,792 \\
\hline 13 & Lead Recovery Subsystem & & & & & \\
\hline 14 & Air Pollution Control Subsystem & & 955 & 1,453 & 1,320 & 1,678 \\
\hline 15 & Polymer Stabilization Subsystem & & 2,322 & 3,534 & 3,210 & 4,080 \\
\hline 16 & Grout Stabilization Subsystem & & 1,931 & 2,940 & 2,670 & 3,393 \\
\hline 17 & Certification \& Shipping Subsystem & & 28,277 & & & 12,725 \\
\hline \multirow[t]{3}{*}{18} & Support Subsystem & 3,360 & 16,883 & & & 9,109 \\
\hline & Total Cost & & & & & 75,617 \\
\hline & & \multicolumn{4}{|c|}{ Post Totals To Table 4-5, Item } & 7.0 \\
\hline
\end{tabular}




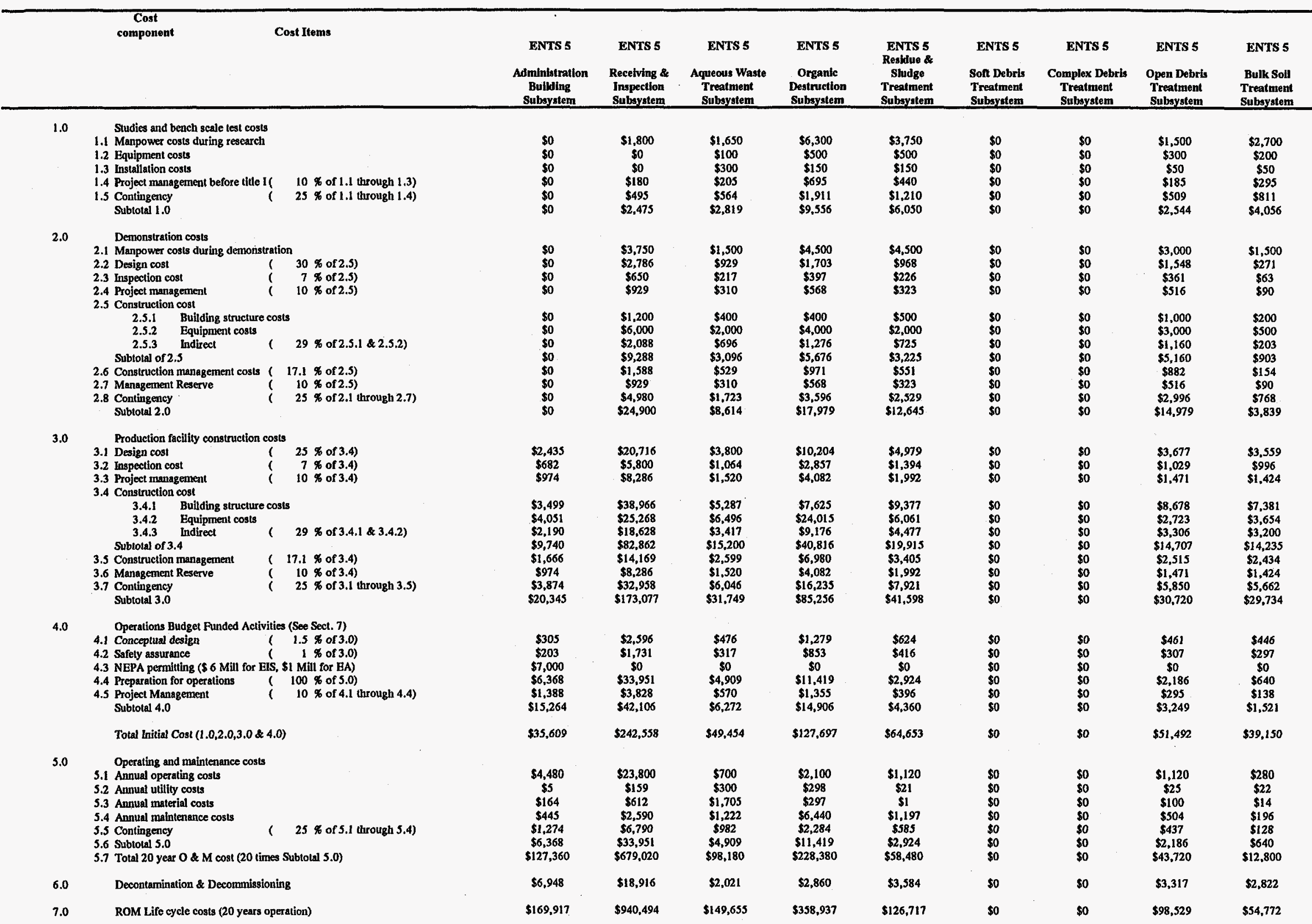


Table B-5.6 : Total Life-Cycle Cost (without Disposal) Estimate Summary for

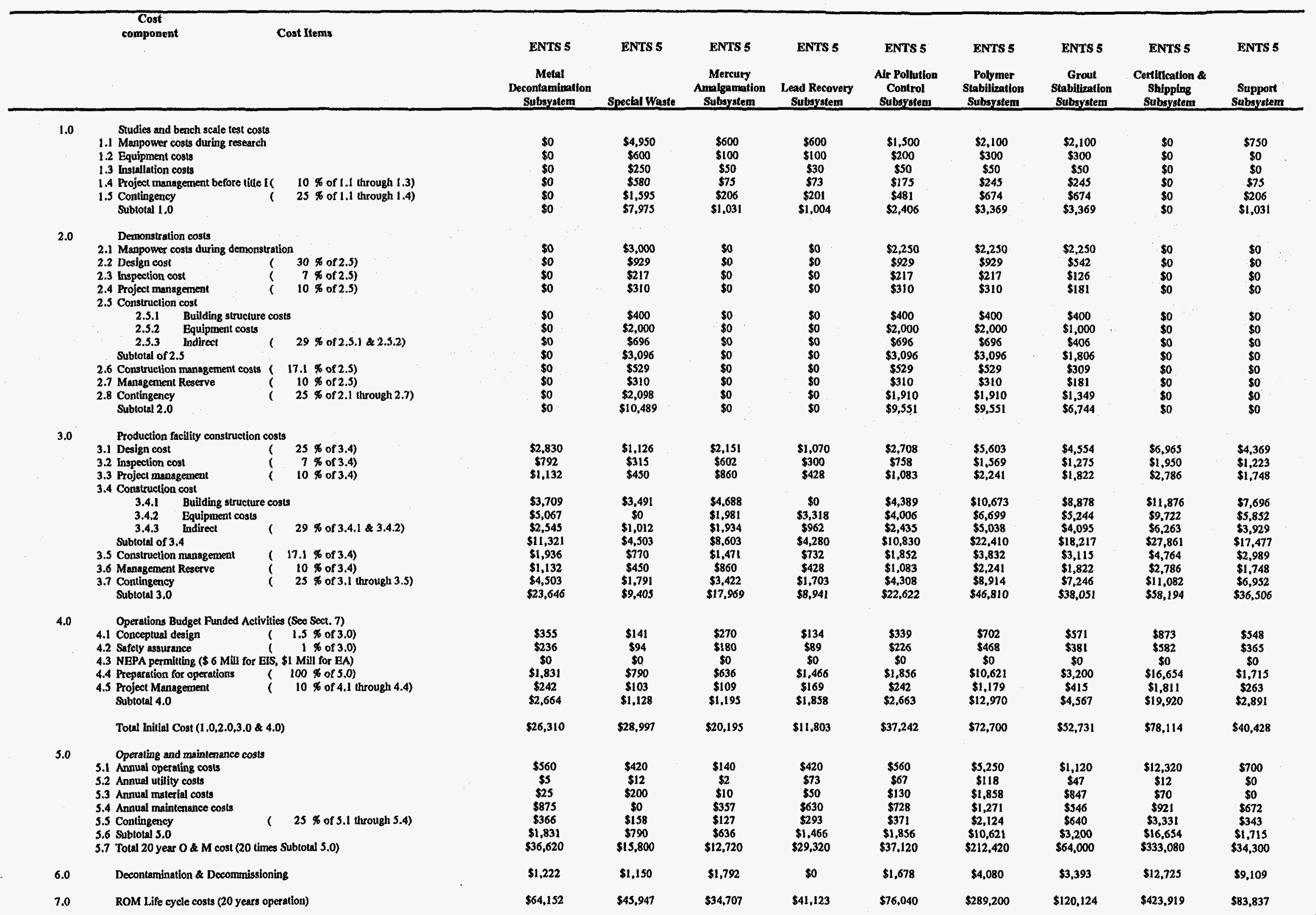

